\begin{abstract}
ADVANCED BEAMLINE DESIGN FOR FERMILAB'S ADVANCED SUPERCONDUCTING TEST ACCELERATOR
\end{abstract}

\author{
Christopher R. Prokop, Ph.D. \\ Department of Physics \\ Northern Illinois University, 2014 \\ Philippe Piot, Director
}

The Advanced Superconducting Test Accelerator (ASTA) at Fermilab is a new electron accelerator currently in the commissioning stage. In addition to testing superconducting accelerating cavities for future accelerators, it is foreseen to support a variety of Advanced Accelerator R\&D (AARD) experiments. Producing the required electron bunches with the expected flexibility is challenging.

The goal of this dissertation is to explore via numerical simulations new accelerator beamlines that can enable the advanced manipulation of electron bunches. The work especially includes the design of a low-energy bunch compressor and a study of transverse-to-longitudinal phase space exchangers. 
NORTHERN ILLINOIS UNIVERSITY

DE KALB, ILLINOIS

MAY 2014

\section{ADVANCED BEAMLINE DESIGN FOR FERMILAB'S ADVANCED SUPERCONDUCTING TEST ACCELERATOR}

BY

CHRISTOPHER R. PROKOP

(C) 2014 Christopher R. Prokop

A DISSERTATION SUBMITTED TO THE GRADUATE SCHOOL

IN PARTIAL FULFILLMENT OF THE REQUIREMENTS

FOR THE DEGREE

DOCTOR OF PHILOSOPHY

DEPARTMENT OF PHYSICS

Director:

Philippe Piot 


\section{ACKNOWLEDGEMENTS}

There were many coworkers and collaborators that helped with the work contained in this dissertation. I would in particular like to acknowledge the developers of the various simulation codes that are used extensively throughout, including Ji Qiang for his work on IMPACT, and Martin Dohlus and Torsten Limberg for their work on CSRTRACK. I would especially like to thank Michael Borland and the others at Argonne that have developed ELEGANT. It has been, by far, the most user-friendly software for accelerator physics, its open-source nature has been tremendously helpful, and the help forum is among the best I've ever used.

I would also like to thank Marwan Rihaoui, my advisor's first Ph.D. student. Even though his research was performed at Argonne rather than Fermilab, I view him as my predecessor, and much of my research follows from his earlier work.

Special thanks to Bruce Carlsten at Los Alamos, without whom none of this research would have been possible. His constant support was vital to this project's success, and I hope that the research contained herein proves useful for the future of Los Alamos' MARIE facility.

Lastly, I would like to thank my advisor, Philippe Piot, for his constant support and encouraging attitude.

This work was supported by Los Alamos LDRD project \#20110067DR, by the U.S. Department of Energy Contract No. DE-FG02-08ER41532 with Northern Illinois University, and No. DE-AC02-07CH11359 with Fermilab. 


\section{DEDICATION}

Dedicated to my friends and family for their frequent encouragement toward finishing. I needed it. 


\section{TABLE OF CONTENTS}

Page

LIST OF TABLES ....................... vii

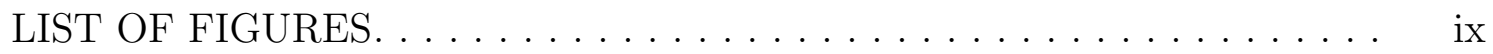

LIST OF APPENDICES ....................... $\ldots \ldots$ xvi

Chapter

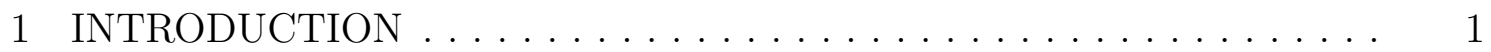

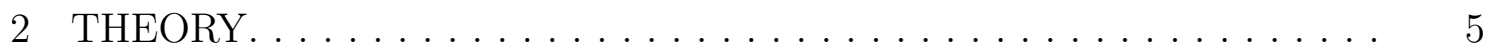

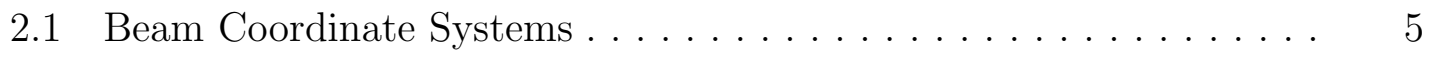

2.2 Courant-Snyder Parameters . . . . . . . . . . . . . . . . . . . 10

2.3 Emittance and Eigen Emittances . . . . . . . . . . . . . . . 11

2.4 Beam Dynamics and Transport . . . . . . . . . . . . . . 15

2.5 Bunch Compression. . . . . . . . . . . . . . . . 16

2.6 Collective Effects. . . . . . . . . . . . . . . . . . 17

2.7 Wakefields .......................... 20

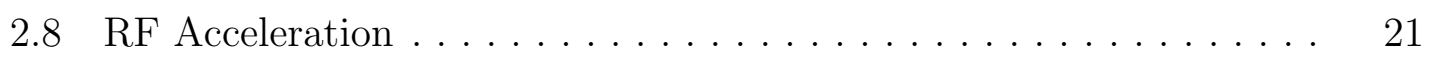

3 THE ADVANCED SUPERCONDUCTING TEST ACCELERATOR . . . 23

3.1 The ASTA Facility . . . . . . . . . . . . . . . . 23

3.2 Planned Advanced Accelerator Research and Development . . . . . . 28

3.3 First Beam Design of the High-Energy Transport Line . . . . . . . . . 29

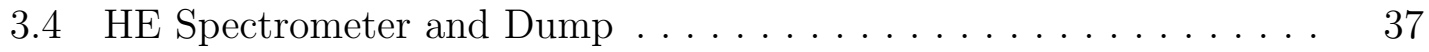

3.5 Performance Studies . . . . . . . . . . . . . . . . . . . . 39 
Chapter Page

3.6 Transverse emittance measurements . . . . . . . . . . . . . . . . . 41

3.7 Quadrupole Magnet Scan Experiment at A0 . . . . . . . . . . 45

4 NUMERICAL STUDIES OF LOW-ENERGY BUNCH COMPRESSION

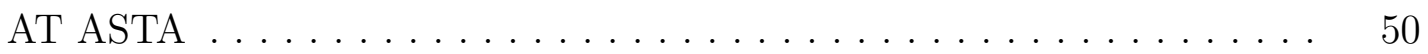

4.1 Introduction to Bunch Compression and Bunch Compressor Design . 50

4.2 Longitudinal Beam Dynamics in the Injector . . . . . . . . . . . . . . 52

4.3 Bunch Compressor Beam Optics. . . . . . . . . . . . . 56

4.4 Code Comparisons and Parametric Studies . . . . . . . . . . . . . 65

4.5 Benchmarking of numerical models . . . . . . . . . . . . . . . 68

4.6 Expected beam dilution and trade-offs . . . . . . . . . . . . 71

4.7 High-Energy Limit . . . . . . . . . . . . . . . . . . . . 73

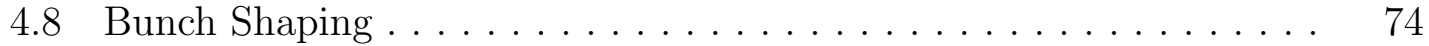

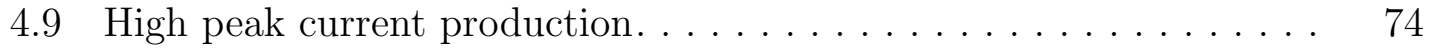

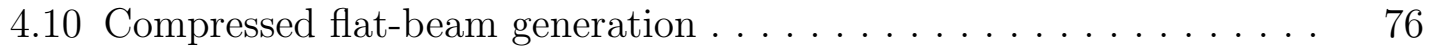

4.11 Double-bunch generation . . . . . . . . . . . . . . . . 78

4.12 BC1 Summary . . . . . . . . . . . . . . . . . . . . 79

5 LONGITUDINAL PHASE SPACE MEASUREMENT . . . . . . . . . . . . . . 94

5.1 Beamline Design . . . . . . . . . . . . . . . . . . . 94

5.2 Transverse Collimation . . . . . . . . . . . . . . . . 97

5.3 Resolution . . . . . . . . . . . . . . . . . . . . . . 100

5.4 Testing at HBESL . . . . . . . . . . . . . . . . . . . . . . . 102

6 A DESIGN OF AN IN-LINE TRANSVERSE-TO-LONGITUDINAL EMIT-

TANCE EXCHANGER. . . . . . . . . . . . . . . . . . . . . . . 106

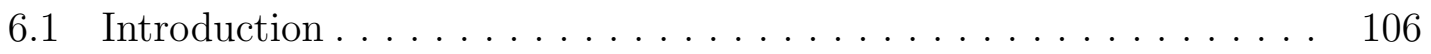

6.2 Basics of Phase-space-Exchanging beamlines . . . . . . . . . . . . . . 109 
Chapter Page

6.3 Simulation Methods . . . . . . . . . . . . . . . . . . . . . . 113

6.3 .1 Overview ............................. 113

6.4 Overview of EEX Designs . . . . . . . . . . . . . . . . . . . . 114

6.4.1 Conventional double-dogleg emittance-exchange (DDEEX) beamline . . . . . . . . . . . . . . . . . . . 115

6.4.2 Chicane-like, nominal dispersion, emittance-exchange (NDEEX)

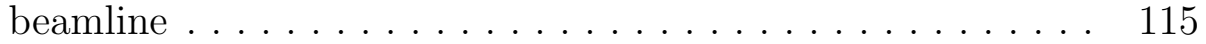

6.4.3 Chicane-like, boosted dispersion, emittance-exchange (BDEEX)

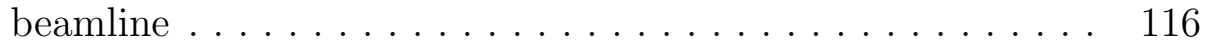

6.5 Note on TDC models. . . . . . . . . . . . . . . . . . . . . . . . . 117

6.6 First-Order Comparison of different EEX designs . . . . . . . . . . . . . 119

6.7 DDEEX vs NDEEX in ELEGANT . . . . . . . . . . . . . . . . 123

6.8 Comparative Studies in IMPACT-Z. . . . . . . . . . . . . . . . . 126

6.9 Code Benchmarking . . . . . . . . . . . . . . . . . . . . 132

6.10 Dispersion Boosting. . . . . . . . . . . . . . . . . . . . 133

6.11 Shaping Studies . . . . . . . . . . . . . . . . . . . . 134

6.12 High Energy Variant . . . . . . . . . . . . . . . . . . . . 137

6.13 Double Emittance Exchanger . . . . . . . . . . . . . . . . . . . 139

6.14 Conclusion . . . . . . . . . . . . . . . . . . . . . . . . . . . . 143

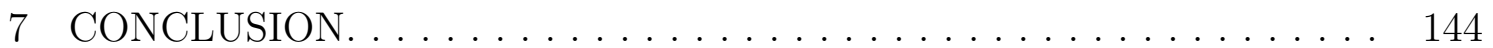

REFERENCES . . . . . . . . . . . . . . . . . . . . . . . . 146

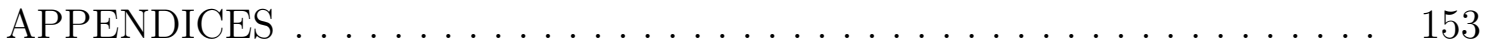




\section{LIST OF TABLES}

Table

Page

3.1 Parameters concerning the FODO lattice. . . . . . . . . . . . . 31

3.2 Data from A0 quadrupole scan. . . . . . . . . . . . . . . . . 48

4.1 Transverse and longitudinal beam parameters 0.1-m upstream of B1 dipole entrance face. Only the Courant-Snyder parameters were fixed while the other parameters depend on the bunch charge or upstream beamline settings. . . . . . . . . . . . . . . .

4.2 Initial normalized transverse $\varepsilon_{x / y, i}$ and longitudinal $\varepsilon_{z, i}$ emittances and RMS bunch length $\sigma_{z, i}$ for the four cases of charge considered in this paper. The parameters are computed 0.1-m upstream of dipole magnet B1's entrance face. . . . . . . . . . . . . . . . . .

4.3 Simulated beam parameters downstream of BC1 with IMPACT-Z ("IMPZ") and CSRTRACK ("CSRT") the model used is appended to the program's name. The parameter column and indicates the number of bins, or the absolute or relative (in \%) Gaussian particle size in the longitudinal dimension, $\sigma_{\|}$. These entries were generated for $\mathcal{C}=$ at $5.22 \mathrm{~m}^{-1}$, so that the bunch is near maximum compression.

4.4 Final normalized longitudinal $\varepsilon_{z}$ at maximum compression $(\mathcal{C}=$ $\left.5.2 \mathrm{~m}^{-1}\right)$ simulated with impact-z (SC+CSR) and CSRTRACK (P2P).

4.5 Initial parameters for different aspect ratios. . . . . . . . . . .

5.1 Comparison of bunch and beamline parameters relevant to the LPS measurement system.. . . . . . . . . . . . . . . . . . 103

6.1 Two sets of initial beam parameters. . . . . . . . . . . . . . . . . 114

6.2 Beamline parameters of the EEX configurations in Figure 6.1 . . . 116 
6.3 Quality of Emittance Exchange for various simulations, varying configuration, simulation order, model for TDC+ACC cavities, and which emittances/lengths are used ("2" uses shorter bunches and lower emittances than " $1 ") \ldots \ldots \ldots \ldots \ldots \ldots \ldots$

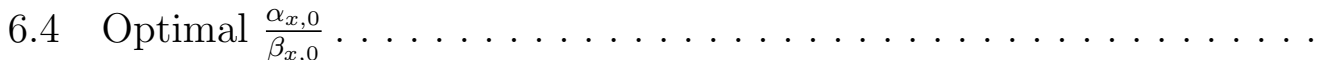

6.5 Exchange quality for various simulations for the NDEEX, at $\beta_{x, 0}=5 \mathrm{~m}$

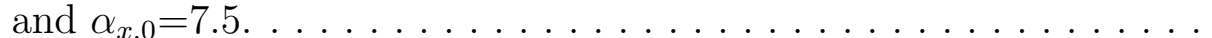

6.6 Quality of Emittance Exchange for various dispersions using the BDEEX configuration, along with which emittances were used ("A0" is shorter bunch and lower emittance than "ASTA") . . . . . . . . . 136

6.7 Initial beam parameters for the test microbunch train used for the Double EEX simulations. . . . . . . . . . . . . . . . . . . . . .

C.1 Number of macroparticles $N$ used as a function of the number of longitudinal bins $N_{z} \ldots \ldots \ldots \ldots \ldots \ldots \ldots \ldots \ldots \ldots \ldots \ldots \ldots \ldots \ldots$

C.2 Scan of Variable Size. . . . . . . . . . . . . . . . . . . . . 185

C.3 Initial transverse emittances for the charges used. . . . . . . . . . . . 187

C.4 Descriptions of particle formats for inter-code compatibility . . . . . 188 


\section{LIST OF FIGURES}

$\begin{array}{lll}\text { Figure } & \text { Page }\end{array}$

2.1 Coordinate system used for accelerator physics. . . . . . . . . 6

2.2 Sample horizontal (left), vertical (middle) and longitudinal (right) trace spaces used for several simulations of ASTA. . . . . . . . . . 7

2.3 An example schematic of a chicane bunch compressor. . . . . . . . . 16

2.4 Characteristic forces of CSR in a dipole (left) and LSC in a drift

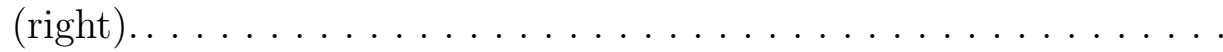

3.1 Full overview of one possible configuration of the ASTA beamline, divided into three regions. . . . . . . . . . . . . . . . . . . 24

3.2 Picture of the ASTA electron source gun from Reference [?]. . . . . 25

3.3 A picture of a bare niobium nine-cell cavity. . . . . . . . . . . 26

3.4 Schematic diagram of the two spectrometers. . . . . . . . . . . . . 27

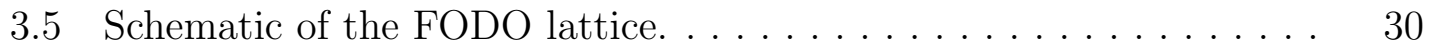

3.6 Maximum (left) and minimum (right) values of the betatron function in the FODO lattice as functions of the betatron phase advance. 32

3.7 Maximum (left) and minimum (right) acceptable values for the betatron function.. . . . . . . . . . . . . . . . . .

3.8 Lattice functions inside the cryomodule for different operating phases. 34

3.9 Contour plots for $\alpha_{f}$ (upper left), $\beta_{f}$ (upper right) and transmission through the cryomodule (lower plot) at the cryomodule exit for the nominal initial C-S parameters. . . . . . . . . . . . . . 
Figure

Page

3.10 Contour plots for $\alpha_{f}$ (upper left), $\beta_{f}$ (upper right) and transmission through the cryomodule (lower plot) at the cryomodule exit for the nominal initial C-S parameter, with ACC1 operated at $\phi=30^{\circ}$ off-crest. . . . . . . . . . . . . . . . . . . . . . . . .

3.11 Contour plots for $\alpha_{f}$ (upper left), $\beta_{f}$ (upper right) and transmission through the cryomodule (lower plot) at the cryomodule exit for the nominal initial C-S parameters, with ACC1 operated $\phi=60^{\circ}$ offcrest. . . . . . . . . . . . . . . . . . . . . . . . . . .

3.12 Contour plots for $\alpha_{f}$ (upper left), $\beta_{f}$ (upper right) and transmission through the cryomodule (lower plot) at the cryomodule exit for the nominal initial C-S parameters $(\alpha, \beta)=(0.5,11 \mathrm{~m})$, with $\mathrm{ACC} 1$ operated $\phi=90^{\circ}$ off-crest. . . . . . . . . . . . . . . . . 36

3.13 Evolution of the betatron functions $\beta_{x}$ (red) and $\beta_{y}$ (blue)...... 37

3.14 Final relative fractional energy spread $\sigma_{\delta f}$ as a function of off-crest

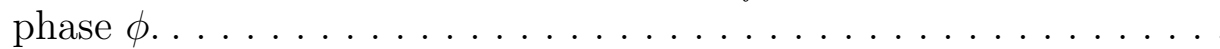

3.15 Betatron functions along the beamline, with ACC1 operated on-

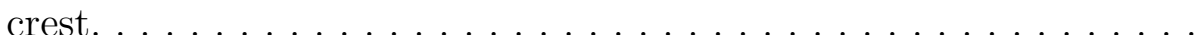

3.16 Betatron functions along the beamline, with ACC1 operated thirtydegrees off-crest. . . . . . . . . . . . . . . . . . . . .

3.17 Betatron functions along the beamline, with ACC1 operated sixtydegrees off-crest. . . . . . . . . . . . . . . . . .

3.18 RMS beam size evolution as a function of scanned quadrupole strength. 43

3.19 Relative error on measured emittance.. . . . . . . . . . . . . . . . 44

3.20 Histograms of 5000 emittance measurements. . . . . . . . . . . . 45

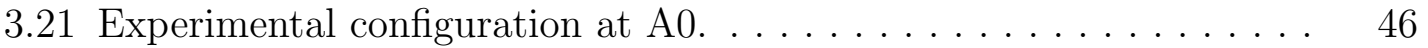

3.22 Images taken from the screen via the capture software.. . . . . . . 46

3.23 Data from the A0 quadrupole magnet scan experiment, RMS beam size vs. K1 setting of the quadrupole magnet in units of $\frac{1}{m^{2}} \ldots \ldots$.

4.1 Injector configuration at ASTA. . . . . . . . . . . 
Figure

Page

4.2 The operating phase $\phi_{39}$ (left) and voltage $V_{39}$ (right) for CAV39 as functions of the operating phase of CAV $2, \phi_{2}$, using its voltage

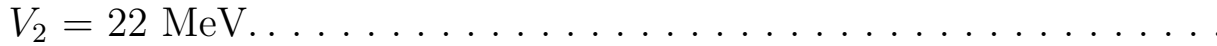

4.3 LPS distributions (top row) and associated current profiles (bottom row) before (left column) and after (right column) BC1, without collective effects. $\ldots \ldots \ldots \ldots \ldots \ldots \ldots \ldots . \ldots \ldots$

4.4 Contour plot of the final normalized horizontal emittance $\left(\varepsilon_{x}\right.$ in $\left.\mu \mathrm{m}\right)$ a function of the initial C-S parameters $\beta_{i}$ and $\alpha_{i}$ for the nominal (left) and linearized (right) incoming LPS.. . . . . . . . . . . . . .

4.5 Evolution of the horizontal (dashed line) and vertical (dotted line) betatron functions through the ASTA injector. . . . . . . . . . .

4.6 LPS distributions 0.1-m upstream of dipole magnet B1's entrance face for $3.2(\mathrm{a}), 1.0(\mathrm{~b}), 0.25$ (c) and $0.02 \mathrm{nC}(\mathrm{d})$ bunches. . . . . . .

4.7 LPS downstream of the bunch compressor and associated longitudinal charge distribution (red trace) in arbitrary units using CSRTRACK's P2P model $\ldots \ldots \ldots \ldots \ldots \ldots \ldots \ldots \ldots$

4.8 RMS bunch length $\sigma_{z}$ downstream of BC1 as a function of the LPS chirp for various bunch charges. . . . . . . . . . . . .

4.9 LPS at the end of the bunch compressor and associated longitudinal charge distribution (red trace) in arbitrary units ...........

4.10 LPS at BC1 exit for IMPACT-Z's (a-d) and CSRTRACK's (e-h) 3D models. . . . . . . . . . . . . . . . . . . . . . . .

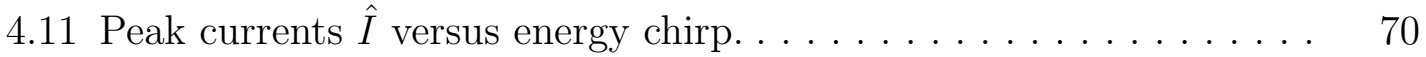

4.12 Final horizontal emittances for each of the different bunch charges. . 80

4.13 Final vertical emittances for each of the different bunch charges. . .

4.14 Example of final normalized transverse slice emittances (top row) and energy spread (bottom row) evolution within a 1-nC bunch for

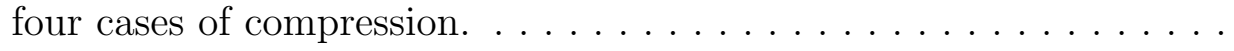

4.15 Final normalized transverse slice emittances $\varepsilon_{x, M}$ in the slice with the highest peak current. . . . . . . . . . . . . . . . 
Figure

4.16 Peak transverse brightness $B_{\perp}=\frac{\hat{I}}{4 \pi^{2} \varepsilon_{x} \varepsilon_{y}}$ versus energy chirp . . . . . .

4.17 Maximum peak transverse brightness $B_{\perp}=\frac{\hat{I}}{4 \pi^{2} \varepsilon_{x} \varepsilon_{y}}$ versus bunch charge

4.18 Final normalized transverse emittances $\varepsilon_{x}$ versus peak currents. . . .

4.19 Final transverse emittance $\varepsilon_{x}(\mu \mathrm{m})$ versus LPS chirp..........

4.20 Final transverse emittances $\varepsilon_{x}$, relative emittance growths $\frac{\varepsilon_{x}}{\varepsilon_{y, 0}}$, bunch lengths $\sigma_{z}$, and peak currents at the exit of $\mathrm{BC} 1$ as functions of the

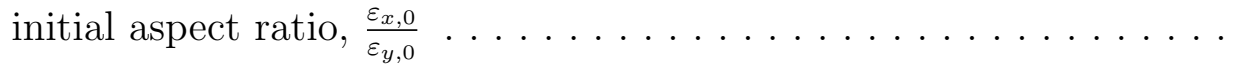

4.21 LPS and associated longitudinal charge distribution (red trace) in arbitrary units (left) and current profile (right) for a $3.2-\mathrm{nC}$ bunch over-compressed with $\mathcal{C}=5.50 \mathrm{~m}^{-1}$, simulated in IMPACT-Z. . . . . .

4.22 Bunch form factor associated to the 3.2-nC fully-compressed electron bunch (left plot) and final horizontal (circles), vertical (triangles), and longitudinal (squares) normalized emittances (left vertical axis) and peak current (dashed lines and dots, right axis) of the bunch within a selected transverse radius (right plot). . . . . . . .

4.23 Final LPS for initial flat beam aspect ratios of 1 (a), 25 (b), 100 (c),

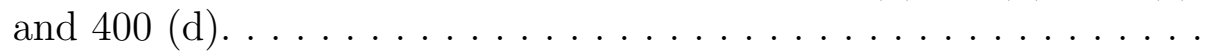

4.24 Final $x$-y profiles for initial aspect ratios of 1 (a), 25 (b), 100 (c),

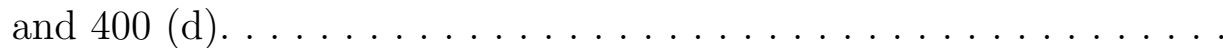

4.25 Bending plane transverse emittance $\frac{\varepsilon_{x}}{\varepsilon_{x, i}}$ growth in $\mathrm{BC} 1 \ldots \ldots \ldots$

4.26 LPS distribution (gray colormap in left plot) and current projection (lower trace and right plot) associated to over-compressed bunches with an incoming LPS chirp of $\mathcal{C}=5.5 \mathrm{~m}^{-1} \ldots \ldots \ldots \ldots$

5.1 Overview of the LPS diagnostic section with associated horizontal (blue) and vertical (red) betatron function. . . . . . . . . .

5.2 The beam profile at the screen (top row) and reconstructed LPS

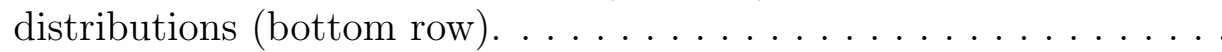


5.3 LPS distributions (top row) generated using AsTRA after BC1 (left column), reconstructed from the screen without transverse collimation (middle column), and reconstructed with collimation using a 100- $\mu \mathrm{m}$ square aperture (right column), with each LPS's current profile beneath it in the bottom row.. . . . . . . . . . . . . .

5.4 Area-normalized fractional-energy-spread histograms of macroparticles located in the center-most $180-\mu \mathrm{m}$ longitudinal slice of the reconstructed LPS for a chirped Gaussian beam, for square collimators with sizes of 50 (blue), 140 (green), 387 (red), 1000 (cyan), and 3000 (magenta) $\mu \mathrm{m}$, and the pre-TDC LPS (yellow). . . . . . . . .

5.5 Longitudinal-coordinate (left) and fractional energy spread (right) resolutions as a function of initial normalized emittances, $\varepsilon_{x}=\varepsilon_{y}$, for different collimation scenarios (see legend). . . . . . . . . . . . . 101

5.6 Generic layout of the LPS diagnostic system . . . . . . . . . . . . . . 102

5.7 Photograph of cross X07 illuminated with a light bulb. . . . . . . 103

5.8 Scan of centroid offset versus TDC phase at A0 during original commissioning of the TDC. . . . . . . . . . . . .

6.1 The DDEEX (top) and chicane-like (bottom) emittance-exchanger configurations. . . . . . . . . . . . . . . . . . . . 108

6.2 Contour plot of $\mathcal{F}_{x \rightarrow z}(\mathrm{a}, \mathrm{b}, \mathrm{c})$ and $\mathcal{F}_{z \rightarrow x}(\mathrm{~d}, \mathrm{e}, \mathrm{f}) \ldots \ldots \ldots$

6.3 Contour plot of final bunch lengths $\sigma_{z}(\mathrm{a}, \mathrm{d}), \mathcal{F}_{x \rightarrow z}(\mathrm{~b}, \mathrm{e})$ and $\mathcal{F}_{z \rightarrow x}$ $(\mathrm{c}, \mathrm{f})$ as a function of the initial $x \mathrm{C}-\mathrm{S}$ parameters.. . . . . . .

6.4 Contour plot of final vertical parameters $\sigma_{y}(\mathrm{a}, \mathrm{d}), \mathcal{F}_{x \rightarrow z}(\mathrm{~b}, \mathrm{e})$ and $\mathcal{F}_{z \rightarrow x}(\mathrm{c}, \mathrm{f})$ as a function of the initial $y$ C-S parameters for the NDEEX $(\mathrm{a}-\mathrm{c})$ and DDEEX $(\mathrm{d}-\mathrm{f})$ designs. . . . . . . . . . .

6.5 Contour plots of $F_{z \rightarrow x} \operatorname{NDEEX}(\mathrm{a}-\mathrm{d})$ and DDEEX (e-h) designs in ELEGANT (a,e) and Impact-Z for $0 \mathrm{nC}(\mathrm{b}, \mathrm{f}), 1 \mathrm{nC} \mathrm{SC}(\mathrm{c}, \mathrm{g})$, and $1 \mathrm{nC} \mathrm{SC}+\mathrm{CSR}(\mathrm{d}, \mathrm{h})$ as functions of the C-S parameters at the EEX entrance, $\beta_{x, 0}$ and $\alpha_{x, 0} \ldots \ldots \ldots \ldots \ldots \ldots \ldots \ldots \ldots \ldots \ldots \ldots \ldots \ldots \ldots$ 
6.6 Contour plots of $\mathcal{F}_{x \rightarrow z} \operatorname{NDEEX}(\mathrm{a}-\mathrm{d})$ and DDEEX (e-h) designs in ELEGANT (a,e) and Impact-Z for $0 \mathrm{nC}(\mathrm{b}, \mathrm{f}), 1 \mathrm{nC} \mathrm{SC}(\mathrm{c}, \mathrm{g})$, and $1 \mathrm{nC} \mathrm{SC}+\mathrm{CSR}(\mathrm{d}, \mathrm{h})$ as functions of the C-S parameters at the EEX

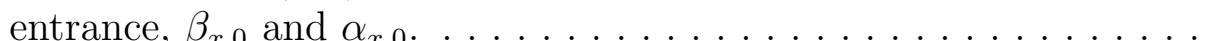

6.7 Horizontal (blue), vertical (green), and longitudinal (red) RMS beam sizes along the EEX for C-S parameters of $\alpha_{x, 0}, \beta_{x, 0}=7.5,10 \mathrm{~m}$ (a),

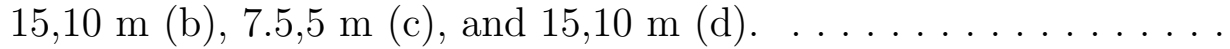

6.8 Contour plots comparison of three models (DDEEX (a,d), NDEEX $(\mathrm{b}, \mathrm{e})$, and $\operatorname{BDEEX}(\mathrm{c}, \mathrm{f}))$ with IMPACT-Z for $0 \mathrm{nC}$ for both $\mathcal{F}_{x \rightarrow z}$ (ac) and $\mathcal{F}_{z \rightarrow x}(\mathrm{~d}-\mathrm{f})$, as functions of the C-S parameters at the EEX

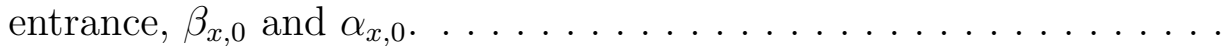

6.9 Contour plots comparison of three models (DDEEX, NDEEX, and BDEEX) with IMPACT-Z for $0 \mathrm{nC}$ for both $\mathcal{F}_{z x}$ and $\mathcal{F}_{x z}$, as functions of the C-S parameters at the EEX entrance, $\beta_{x, 0}$ and $\alpha_{x, 0} \ldots \ldots \ldots$

6.10 Bunch size (left), emittances (middle) and Debye length over RMS bunch size (right) for the NDEEX design simulated with IMPACT-Z with 1nC SC+CSR, with $x$ (blue), $y$ (red) and $z$ (green) plotted together. . . . . . . . . . . . . . . . . . . . . . .

6.11 Horizontal dispersion $\eta_{x}$ (left), $\sigma_{x}$ (middle), and $\sigma_{y}$ (right) along the NDEEX and BDEEX designs for $\eta_{-}=0.5 \mathrm{~m}$ (blue), $1.0 \mathrm{~m}$ (red), $1.5 \mathrm{~m}$ (green), $2.0 \mathrm{~m}$ (magenta), $2.5 \mathrm{~m}$ (yellow) and $3.0 \mathrm{~m}$ (black). .

6.12 Horizontal dispersion $\eta_{x}$ (left), $\sigma_{x}$ (middle), and $\sigma_{y}$ (right) along the NDEEX and BDEEX designs for $\eta_{-}=0.5 \mathrm{~m}$ (blue), $1.0 \mathrm{~m}$ (red), $1.5 \mathrm{~m}$ (green), $2.0 \mathrm{~m}$ (magenta), $2.5 \mathrm{~m}$ (yellow) and $3.0 \mathrm{~m}$ (black).. .

6.13 Pre-collimated (Upper Left), post-collimated (Upper Right) transverse profile, and post-EEX LPS without (lower left) and with lower right) collective effects, with projection of the $x$-axis shown in red. .

6.14 Post-EEX normalized density profiles for various levels of collective

6.15 Contour plots comparison with IMPACT-Z for $0 \mathrm{nC}(\mathrm{a}, \mathrm{d}), 1 \mathrm{nC} \mathrm{SC}$ (b,e), and $1 \mathrm{nC} \mathrm{SC}+\mathrm{CSR}(\mathrm{c}, \mathrm{f})$ with both $\mathcal{F}_{x \rightarrow z}(\mathrm{a}-\mathrm{c})$ and $\mathcal{F}_{z \rightarrow x}(\mathrm{~d}-\mathrm{f})$, as functions of the C-S parameters at the EEX entrance, $\beta_{x, 0}$ and $\alpha_{x, 0}$, at a beam energy of $250 \mathrm{MeV} \ldots \ldots \ldots \ldots$ 
Figure

6.16 Double emittance exchanger beamline (Top) with horizontal (blue), vertical (red) and longitudinal (green) RMS beam sizes, with schematic overlay. . . . . . . . . . . . . . . . . . . . . . . . . . . . 142

C.1 LPS density plots at BC1 exit in IMPACT-Z varying the number of longitudinal space-charge bins $N_{z}$, while keeping the number of particles per bin constant to $N /\left(N_{x} N_{y} N z\right)=5$, with $N_{z}$ set to 64 (a), 128 (b), 256 (c), 512 (d), 1024 (e) and 2048 (f) bins. Adjusting the $N_{z}$ has little impact on the longitudinal dynamics for these simulations. . . . . . . . . . . . . . . . . . . . .

C.2 Current profiles at BC1 exit in IMPACT-Z varying the number of longitudinal space-charge bins, while keeping the number of particles per bin constant by increasing the number of particles in the distribution, with $N_{z}$ set to 64 (red), 128 (blue), 256 (green), 512 (magenta), 1024 (yellow) and 2048 (black) . . . . . . . . . . . .

C.3 Variance of $\mathrm{I}_{N_{z}}-\mathrm{I}_{64}$ for the data shown in FigureC.2, where $\mathrm{N}_{z}$ is the number of longitudinal bins. Compared to the scale of the peak current, this variance is well under $1 \% \ldots \ldots \ldots \ldots \ldots$

C.4 $\varepsilon_{x}$ BC1 exit in IMPACT-Z varying the number of longitudinal spacecharge bins, $N_{z}$, while keeping the number of particles per bin constant, with $N_{z}$ set to $64,128,256,512,1024$ and 2048 number of

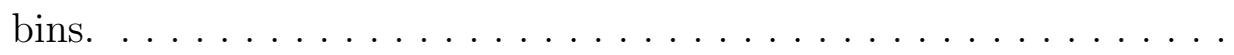

C.5 $\varepsilon_{x}$ BC1 exit in CSRTRACK with the 1DP model versus the size of the Gaussian sub-bunches as a fraction of the RMS length, $\sigma_{z} \ldots \ldots \quad 186$

C.6 Schematic of ASTA denoting which simulation codes are used for different sections. . . . . . . . . . . . . . . . . . . . .

D.1 Schematic of a start-to-end simulation using GlueTrack. This simulation uses five simulations, three read-write pairs, and two "matching" processes. .................... 


\section{LIST OF APPENDICES}

Appendix $\quad$ Page

A TRANSFER MATRICES OF BEAMLINE ELEMENTS . . . . . . . . . . 153

A.1 Basics of Transfer Matrices . . . . . . . . . . . . . . . . . . . . . . 154

A.2 Exceptions to Linear Transfer Maps . . . . . . . . . . . . . . . . . . . . 156

A.3 Transfer Matrices of Common Beamline Components . . . . . . . . . . 157

A.4 Emittance Exchangers and Block Anti-Diagonality. . . . . . . . . . . 161

B DERIVATIONS OF THE TRANSFER MATRIX AND DEFLECTING

KICK OF A TRANSVERSE DEFLECTING CAVITY . . . . . . . . . . . . . 164

C SIMULATION CODE OVERVIEW . . . . . . . . . . . . . . . . . . 171

C.1 Elegant . . . . . . . . . . . . . . . . . . . . . . . 172

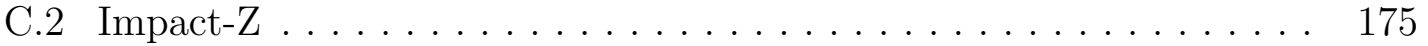

C.3 CSRtrack . . . . . . . . . . . . . . . . . . . . . . . . 179

C.4 CSRtrack Parametric Studies . . . . . . . . . . . . . . . . . . . . . . 183

C.5 Impact-T . . . . . . . . . . . . . . . . . . . . . . . 186

C.6 Comparison of Code Capabilities . . . . . . . . . . . . . . . 187

C.7 Comparison of Particle Formats . . . . . . . . . . . . . . . . . . 187

C.8 Simulation Outline . . . . . . . . . . . . . . . . . . . . . 188

D GLUETRACK: FRAMEWORK FOR MULTI-CODE SIMULATIONS . . 190

D.1 GlueTrack Flow Overview . . . . . . . . . . . . . . . . . . . . . . 191

D.2 Modifications . . . . . . . . . . . . . . . . . . . . . . 195

D.3 Start-to-End Simulations . . . . . . . . . . . . . . . . . . . . . . 196 


\section{CHAPTER 1 INTRODUCTION}

A charged particle beam is an ensemble of particles traveling through vacuum in tandem along a preferred direction at velocities near the speed of light. Beams of charged particles are useful for a variety of scientific, medical, and industrial applications [1]. Scientific experiments involve miles-long proton-accelerator complexes, such as those at CERN [2] and Fermilab [3], down to small table-top electron accelerators such as laser wakefield accelerators [1]. Medical applications include room-sized accelerator for proton and heavy-ion cancer therapies [4]. Industrial and commercial applications range from simple cathode-ray-tube televisions up to the doping of semiconductors [5], as well as uses in technologies related to nuclear power [1].

Charged particle beams are typically bunched, with each bunch comprised of millions or billions of particles traveling together in tandem. These bunches have charges on the order of picocoulombs $(\mathrm{pC})$ or nanocoulombs $(\mathrm{nC})$, and have energies that range from kilo-electron volts $(\mathrm{keV})$ to tera-electron volts $(\mathrm{TeV})$.

A new linear electron accelerator known as the Advanced Superconducting Test Accelerator (ASTA) is currently being commissioned at Fermilab. The ASTA facility is dual-purposed. It serves as a testbed for systems related to the International Linear Collidor (ILC) that is used as the maximum and baseline bunch parameters. It also serves as a research facility for Advanced Accelerator Research and Development (AARD), which is the field of research associated with creating more compact, cheaper, and higher-quality particle accelerators. This includes the testing of entirely new methods of acceleration, such as dielectric wakefield acceleration [6, 7], 
as well as improving existing techniques such as increasing the efficiency of existing superconducting cavities [?]. It also serves as a testbed for new beam manipulation techniques and designs, such as a so-called "phase space exchanger" [8], a design for which is proposed in this dissertation.

The ASTA electron beam will eventually contain bunches of up to 20 billion electrons, which corresponds to around $3.2 \mathrm{nC}$ of electric charge. Particles within the bunch fall within a box on the order of millimeters on each side. Lower bunch charges will be used, based on the stage of development of the facility and its target application.

Each electron in a particle accelerator travels at nearly the speed of light and is a source of electromagnetic (EM) fields that vary depending on the particle energy. At rest, a single charged particle of charge $q$ has an electric field

$$
\mathbf{E}=\frac{q}{|\mathbf{r}|^{2}} \hat{\mathbf{r}}
$$

where $\hat{\mathbf{r}}$ is the radial unit vector, $\mathbf{r}$ is the distance vector, and $|\mathbf{r}|$ is its magnitude. A stationary electron has no magnetic field, but in motion has a magetic field

$$
\mathbf{B}=\frac{q}{c} \frac{\mathbf{v} \times \mathbf{r}}{|\mathbf{r}|^{3}}
$$

where $\mathbf{v}$ is the particle velocity, and $c$ is the speed of light. Under relativistic motion, a single particle's electric field is compressed in the transverse dimension and decompressed in the longitudinal direction of motion by a factor of $\gamma^{2}$ and $\frac{1}{\gamma^{2}}$, respectively [9], where the Lorentz factor $\gamma=\frac{1}{\sqrt{1-\frac{v^{2}}{c^{2}}}}$. These fields may interact strongly with other electrons in the bunch, which degrades the quality of the beam. Worse still, when the electron bunch traverses through a bent trajectory, or near 
conducting surfaces, their radiative fields may produce highly-non-linear self-forces. The so called Lienard-Wiechart field [9] of a moving charge is

$$
\mathbf{E}(\mathbf{r}, \mathbf{t})=\frac{1}{4 \pi \epsilon_{0}}\left(\frac{\mathbf{q}(\mathbf{n}-\boldsymbol{\beta})}{\gamma^{2}(\mathbf{1}-\mathbf{n} \cdot \boldsymbol{\beta})^{3}\left|\mathbf{r}-\mathbf{r}_{\mathbf{s}}\right|^{2}}+\frac{\mathbf{q} \mathbf{n} \times((\mathbf{n}-\boldsymbol{\beta}) \times \dot{\boldsymbol{\beta}})}{\mathbf{c}(\mathbf{1}-\mathbf{n} \cdot \boldsymbol{\beta})^{3}\left|\mathbf{r}-\mathbf{r}_{\mathrm{s}}\right|}\right)_{\mathbf{t}_{\mathbf{r}}}
$$

evaluated at $t_{r}$, the retarded time, where $\mathbf{r}$ and $\mathbf{r}_{s}$ are the position vectors of the witness and source particles, and $\beta=\frac{v}{c}, q$ is the charge of the particle, and $\mathbf{n}$ is the unit vector from the source.

The first term corresponds to the interactions with nearby particles, while the second term gives the radiative fields, which may add coherently [10] for extra enhancement. Accurate modeling of these collective effects and retarded fields are difficult to model and control. The study of accelerator physics is heavily dependent upon the development of techniques to accurately model, measure, and control the particle beam.

The goal of this dissertation is the design of advanced beamline manipulations for the ASTA facility. This is supported by extensive simulations and modeling, and is focused to several distinct studies of various aspects of ASTA. In addition, some supporting experiments and tasks were performed at Fermilab's High-Brightness Electron Source Laboratory (HBESL). Like ASTA, HBESL uses a photoinjector as the source of an electron linac. Due to its relative lack of a superconducting radiofrequency $(\mathrm{SRF})$ cavity, it operates at a lower maximum energy ( $\sim 5 \mathrm{MeV})$ than ASTA, and is a smaller facility that totals 8-12 meters long. Despite its relatively low energy, it served as a suitable test-bed for simulation studies of designs and methods discussed in this dissertation, which will eventually be implemented in future stages of ASTA. 
This dissertation is divided into six chapters that explore in detail the studies and design aspects that I performed for ASTA, in addition to the basics of accelerator theory and the design of the ASTA facility. In Chapter 2, I introduce the relevant beam dynamics formalisms. In Chapter 3, I describe the design of the ASTA facility with emphasis on the optimization of several beamlines. Several experimental studies performed at A0/HBESL are also presented. Chapter 4 focuses on an investigation of the low-energy bunch compression, and its implications for several advanced experiments that may be carried out at the ASTA facility. Chapter 5 explores the behavior of transverse deflecting cavities and a single-shot longitudinal phase space spectrometer that was implemented at A0/HBESL and will be implemented at ASTA. Lastly, in Chapter 6, I present a detailed study of phase space exchanger designs and their potential performances when included in the ASTA beamline.

Several appendices are included. These cover several important topics and background information, such as transfer matrix elements for common beamline elements (Appendix A), a detailed derivation for the transfer matrix of a transverse deflecting cavity (TDC) (Appendix B), an overview of the simulations code used in this dissertation (Appendix C), and details on GLUETRACK, the set of Python scripts that I inherited and developed for use in S2E simulations (Appendix D). 


\section{CHAPTER 2 THEORY}

In this chapter, I discuss the beam dynamics theory and formalism used throughout this dissertation and its associated notations. First, I start with definitions of the basic coordinate system that is often used to describe the motion of the billions of particles that comprise each bunch.

\subsection{Beam Coordinate Systems}

Inside a particle accelerator, billions of particles travel together as a "bunch" in a dominant direction. A theoretical particle that travels along the ideal beam path at the average energy of the bunch may be considered the "reference particle". It is useful to define a six-dimensional coordinate system that describes any given particle's position and velocity relative to that of the reference particle. Such a coordinate system is defined relative to both the position and velocity of the reference particle. There are two such conventions that I will discuss in this dissertation. The first, phase space, is useful from a conceptual perspective, while the trace space is the one that is typically measured.

In both of these conventions, $z$ is the particle's longitudinal displacement from the reference particle, $p_{z}$ is the longitudinal momentum of the particle, $x$ is the horizontal transverse coordinate, and $y$ is the other, vertical transverse coordinate. A sample coordinate system is shown in Figure 2.1. 


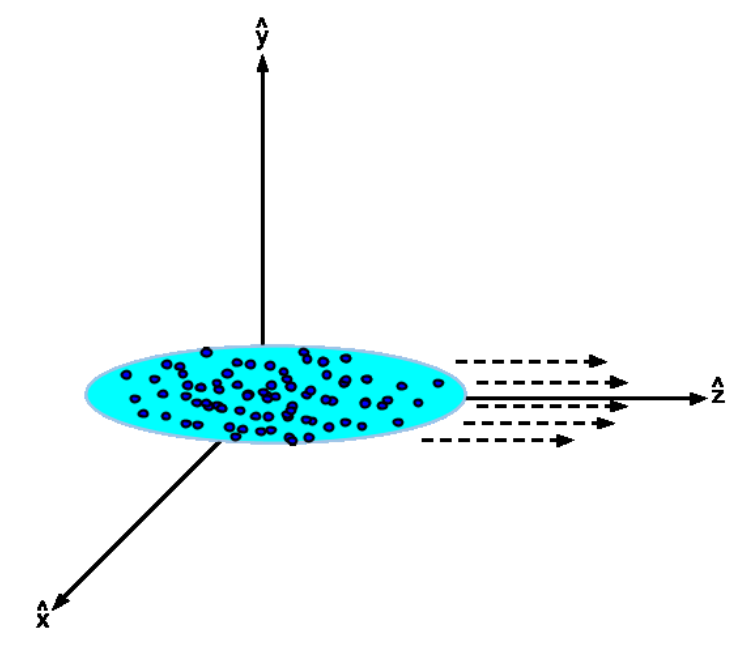

Figure 2.1: Coordinate system used for accelerator physics. The particle bunch moves in the $\hat{z}$ direction, while $\hat{x}$ and $\hat{y}$ are the transverse dimensions.

In the phase space, we used the canonical momenta in each dimension, $p_{x}, p_{y}$, and $p_{z}$. For the trace space formalism, we use slopes in the transverse dimensions, $x^{\prime}$ and $y^{\prime}$, which are normalized to $p_{z}$ such that $x^{\prime}=\frac{p_{x}}{p_{z}}$ and $y^{\prime}=\frac{p_{y}}{p_{z}}$, respectively. Lastly, we use $\delta$ is the fractional momentum deviation of a particle, $\frac{p_{z}-p_{z, r}}{p_{z, r}}$, where the subscript $r$ denotes the value corresponding the reference particle.

A particle's coordinates in the phase space is then represented by

$$
\mathbf{x}_{\mathbf{p s}}{ }^{T} \equiv\left[\begin{array}{llllll}
x & p_{x} & y & p_{y} & z & p_{z}
\end{array}\right]
$$


where $T$ denotes the transpose of the vector. Likewise, the coordinates in trace space, $\mathbf{x}$, are given by

$$
\mathbf{x}^{T} \equiv\left[\begin{array}{llllll}
x & x^{\prime} & y & y^{\prime} & z & \delta
\end{array}\right]
$$

The full ensemble of particles is represented with $\mathbf{X}$. An example of the trace space of a sample distribution is presented in Fig. 2.2.
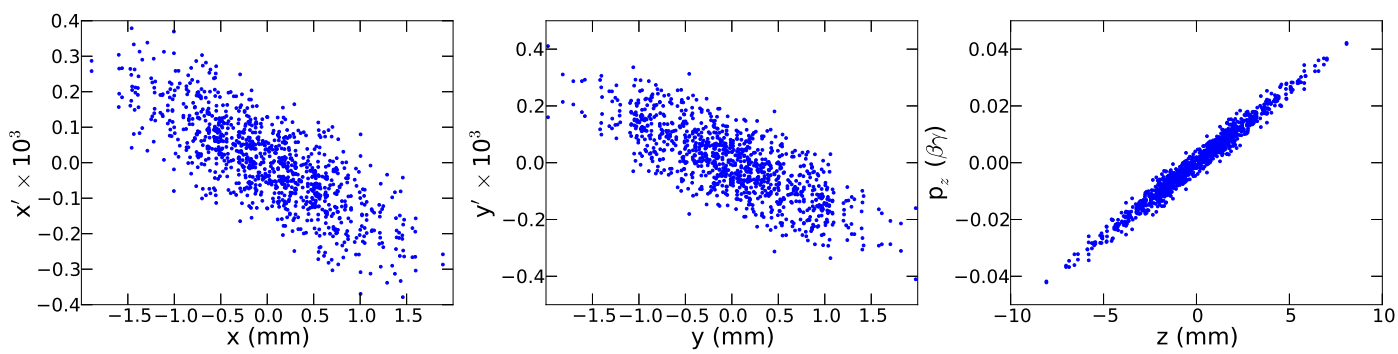

Figure 2.2: Sample horizontal (left), vertical (middle) and longitudinal (right) trace spaces used for several simulations of ASTA.

For this six-dimensional trace-space coordinate system, we can make use of transfer matrices $\mathbf{M}$ to convert from a set of initial coordinates $\overrightarrow{x_{0}}$ to final coordinates $\vec{x}$ via $\overrightarrow{x_{f}}=\mathbf{M} \tilde{\mathbf{x}_{0}}$, or more specifically,

$$
\left[\begin{array}{l}
x \\
x^{\prime} \\
y \\
y^{\prime} \\
z \\
\delta
\end{array}\right]=\left[\begin{array}{llllll}
R_{11} & R_{12} & R_{13} & R_{14} & R_{15} & R_{16} \\
R_{21} & R_{22} & R_{23} & R_{24} & R_{25} & R_{26} \\
R_{31} & R_{32} & R_{33} & R_{34} & R_{35} & R_{36} \\
R_{41} & R_{42} & R_{43} & R_{44} & R_{45} & R_{46} \\
R_{51} & R_{52} & R_{53} & R_{54} & R_{55} & R_{56} \\
R_{61} & R_{62} & R_{63} & R_{64} & R_{65} & R_{66}
\end{array}\right]\left[\begin{array}{c}
x_{0} \\
x_{0}^{\prime} \\
y_{0} \\
y_{0}^{\prime} \\
z_{0} \\
\delta_{0}
\end{array}\right] .
$$

where $R_{i j}$ denote the first-order correlation from initial coordinate $j$ to final coordinate $i$. For more details on this formalism, please see Appendix A. 
The reference particle travels along the ideal path through the particle accelerator. The distance it has traveled is denoted by $s$, and it is common to describe a parameter as a function of $s$, i.e. $E_{r}(s)$ would be the reference particle's energy at position $s$ along the beamline.

The correlation between each of the six dimensions for the entire bunch is described by the beam matrix. This relates the correlations between each of the six dimensions. The triangular brackets \langle\rangle denote the mean value of the enclosed product, i.e. $\langle x y\rangle$ is the average value of each particle's $x$ coordinate multiplied by its $y$ coordinate. Likewise, $\left\langle x^{2}\right\rangle$ is the mean each particles $x$ coordinate squared. The full 6 -by- 6 beam matrix is the covariance matrix,

$$
\Sigma=\langle\mathbf{X} \widetilde{\mathbf{X}}\rangle=\left[\begin{array}{cccccc}
\left\langle x^{2}\right\rangle & \langle x x \prime\rangle & \langle x y\rangle & \left\langle x y^{\prime}\right\rangle & \langle x z\rangle & \langle x \delta\rangle \\
\left\langle x^{\prime} x\right\rangle & \left\langle x^{\prime 2}\right\rangle & \left\langle x^{\prime} y\right\rangle & \left\langle x^{\prime} y^{\prime}\right\rangle & \left\langle x^{\prime} z\right\rangle & \left\langle x^{\prime} \delta\right\rangle \\
\langle y x\rangle & \left\langle y x^{\prime}\right\rangle & \left\langle y^{2}\right\rangle & \left\langle y y^{\prime}\right\rangle & \langle y z\rangle & \langle y \delta\rangle \\
\left\langle y^{\prime} x\right\rangle & \left\langle y^{\prime} x^{\prime}\right\rangle & \left\langle y^{\prime} y\right\rangle & \left\langle y^{\prime 2}\right\rangle & \left\langle y^{\prime} z\right\rangle & \left\langle y^{\prime} \delta\right\rangle \\
\langle z x\rangle & \left\langle z x^{\prime}\right\rangle & \langle z y\rangle & \left\langle z y^{\prime}\right\rangle & \left\langle z^{2}\right\rangle & \langle z \delta\rangle \\
\langle\delta x\rangle & \left\langle\delta x^{\prime}\right\rangle & \langle\delta y\rangle & \left\langle\delta y^{\prime}\right\rangle & \langle\delta z\rangle & \left\langle\delta^{2}\right\rangle
\end{array}\right]
$$

where $\widetilde{\mathbf{X}}$ is the transpose of $\mathbf{X}$. The beam matrix is symmetric and positive definite. For simplicity, this is sometimes represented as a 3-by-3 matrix,

$$
\Sigma=\left[\begin{array}{ccc}
\Sigma_{x x} & \Sigma_{x y} & \Sigma_{x z} \\
\Sigma_{y x} & \Sigma_{y y} & \Sigma_{y z} \\
\Sigma_{z x} & \Sigma_{z y} & \Sigma_{z z}
\end{array}\right]
$$


where each term denotes its respective $2 \times 2$ part of the full beam matrix, i.e.

$$
\Sigma_{x y}=\left[\begin{array}{cc}
\langle x y\rangle & \left\langle x y^{\prime}\right\rangle \\
\left\langle x^{\prime} y\right\rangle & \left\langle x^{\prime} y^{\prime}\right\rangle
\end{array}\right] .
$$

When the three dimensions are uncorrelated $(\langle x y\rangle=0$, etc.), the beam matrix is block diagonal. The beam matrix is then of the form

$$
\Sigma=\left[\begin{array}{ccc}
\Sigma_{x x} & 0 & 0 \\
0 & \Sigma_{y y} & 0 \\
0 & 0 & \Sigma_{z z}
\end{array}\right] .
$$

This demonstrates how having each of the three dimensions uncorrelated from the others leads to drastic simplification of the beam mechanics. The final $x$ and $x^{p}$ rime parameters are functions of only the initial $x$ and $x^{\prime}$, and likewise for $y-y^{\prime}$ and $z-\delta$.

It is often useful to regard only specific aspects of the particle phase space at a time. We refer to the phase space with all six coordinates as the 6D-phase space. Likewise, each dimension has its own 2D phase space, and some combinations of two dimensions are used as 4D phase spaces.

In such cases, the $6 \times 6$ matrix may further be simplified down to smaller $4 \times 4$ matrices- that with only the $\mathrm{x}-\mathrm{y}$ elements $\left(x, x^{\prime}, y, y^{\prime}\right)$, for when discussing the transverse dynamics, or one with only the $\mathrm{x}-\mathrm{z}$ elements $\left(x, x^{\prime}, z, \delta\right)$, when discussing bunch compressors or phase space exchangers. This is valid because two of the dimensions are correlated, while the third is uncorrelated. Similarly, the $2 \times 2$ transfer matrix for a single dimension is sometimes presented.

We use the root-mean-squared (RMS) of the particle distribution in each dimension as its RMS beam size, which is just the distribution's standard deviations, is 
represented as $\sigma_{x, y, z}$. Typically, a bunch's "full-width" length is considered to be between around four and eight RMS sizes.

\subsection{Courant-Snyder Parameters}

The transfer maps described in the previous sections are applicable for singleparticle tracking, but it is useful and oftentimes necessary to analyze the full bunch distribution. This is done by dealing with the evolution of the bunch phase space, and by coming up with a new set of variables that is appropriate in describing that

phase space. The betatron function, denoted by $\beta_{x, y}$ is generalized measure of the beam size, while its partial derivative along the bunch trajectory is given by

$$
\alpha_{x, y}=-\frac{\beta_{x, y}^{\prime}}{2}
$$

where the ' denotes the derivative along the path length, $\frac{d}{d s}$. While the beam parameter known as the emittance is itself described in the following section, it will suffice to state that the betatron function relates to the beam's transverse geometric emittances, $\tilde{\varepsilon}_{x, y}$, and its RMS bunch size as

$$
\sigma_{x, y}=\sqrt{\beta_{x, y} \tilde{\varepsilon}_{x, y}}
$$

Motion of electrons within a bunch are not uniform. Each of the billions of particles has its own relative motion, as represented in the trace space diagrams shown in Figure 2.2. Therefore, it becomes vital to come up with descriptions to characterize the beam. In the previous section, I presented RMS values and the Courant-Snyder (C-S) parameters, i.e., the $\beta$ and $\alpha$ of a bunch. However, there 
are still more valuable ways to describe the beam. $\beta$ and $\alpha$ describe the state of the beam at a particular point, but during beam transport the bunch expands, focuses, and defocuses as it passes through many beamline elements and drifts, and demonstrate nothing of the quality of the particle beam. This demonstrates why it is necessary to develop a statistical representation of the beam quality that remains invariant through basic beam transport.

\subsection{Emittance and Eigen Emittances}

In Hamiltonian systems, the area of the phase space ellipse is conserved [11] as a consequence of Liouville's theorem, making it an ideal quantity to use to use as a figure of merit for the beam quality. If each of the three dimensions are independent from each other (i.e., the beam matrix is block anti-diagonal), the canonical emittances are

$$
\begin{aligned}
\epsilon_{x} & \equiv \frac{1}{m_{e} c} \sqrt{\left\langle x^{2}\right\rangle\left\langle p_{x}{ }^{2}\right\rangle-\left\langle x p_{x}\right\rangle^{2}}, \\
\epsilon_{y} & \equiv \frac{1}{m_{e} c} \sqrt{\left\langle y^{2}\right\rangle\left\langle p_{y}{ }^{2}\right\rangle-\left\langle y p_{y}\right\rangle^{2}}, \text { and } \\
\epsilon_{z} & \equiv \frac{1}{m_{e} c} \sqrt{\left\langle z^{2}\right\rangle\left\langle p_{z}{ }^{2}\right\rangle-\left\langle z p_{z}\right\rangle^{2}} .
\end{aligned}
$$

For an electron beam with a direction of motion that is strongly preferred, we can convert this to our previous defined $6 \mathrm{D}$ coordinate system and make use of the 
approximations $p_{x} \sim x^{\prime}\left\langle p_{z}\right\rangle$ and $\gamma \sim \frac{\left\langle p_{z}\right\rangle}{m_{e} c}$. Likewise, we use the change of coordinate $p_{z} \equiv(\delta+1)\left\langle p_{z}\right\rangle$ to approximate to the normalized emittances,

$$
\begin{aligned}
& \varepsilon_{x} \equiv \beta \gamma \sqrt{\left\langle x^{2}\right\rangle\left\langle x^{\prime 2}\right\rangle-\left\langle x x^{\prime}\right\rangle^{2}}, \\
& \varepsilon_{y} \equiv \beta \gamma \sqrt{\left\langle y^{2}\right\rangle\left\langle y^{\prime 2}\right\rangle-\left\langle y y^{\prime}\right\rangle^{2}}, \text { and } \\
& \varepsilon_{z} \equiv \beta \gamma \sqrt{\left\langle z^{2}\right\rangle\left\langle\delta^{2}\right\rangle-\langle z \delta\rangle^{2}} .
\end{aligned}
$$

The normalized emittances are invariant under acceleration. However, in actuality the transverse beam size of Equation 2.9 focuses (decreases) under acceleration. Removing the factor of $\beta \gamma$ gives the geometric emittances,

$$
\begin{aligned}
& \tilde{\varepsilon}_{x} \equiv \sqrt{\left\langle x^{2}\right\rangle\left\langle x^{\prime 2}\right\rangle-\left\langle x x^{\prime}\right\rangle^{2}}, \\
& \tilde{\varepsilon}_{y} \equiv \sqrt{\left\langle y^{2}\right\rangle\left\langle y^{\prime 2}\right\rangle-\left\langle y y^{\prime}\right\rangle^{2}}, \text { and } \\
& \tilde{\varepsilon}_{z} \equiv \sqrt{\left\langle z^{2}\right\rangle\left\langle\delta^{2}\right\rangle-\langle z \delta\rangle^{2}} .
\end{aligned}
$$

Geometric emittances are the formalism of emittances that actually matters for Equation 2.9, as they are the type of emittances that can be experimentally measured using standard beam diagnostic techniques. However, there is an important shortcoming to such a formalism.

The full six-dimensional emittance is then just the product of the three normalized emittances as

$$
\Gamma=\varepsilon_{x} \varepsilon_{y} \varepsilon_{z}
$$


The beam brightness, $B$, is defined as

$$
B=\frac{Q}{\Gamma}
$$

where $Q$ is the bunch charge. Brightness is an important figure of merit particle accelerators, as it incorporates both the charge and the emittance. We can also define the transverse brightness, using only normalized emittances.

$$
B_{\perp}=\frac{Q}{\varepsilon_{x} \varepsilon_{y}}
$$

This sometimes appears with some normalization factor, which we will introduce later as needed. It is useful for specific cases for which the longitudinal emittance matters less than the transverse emittances.

The importance of emittance and its related values are that it allows us a basic and quantitative measure of the electron beam's quality. It will be used repeatedly throughout this dissertation due to its versatility and importance.

The normalized emittances, as defined in Equations 2.13-2.15, are useful due to their invariance under many beamline elements, such as drifts, quadrupoles, and acceleration. Low-emittance beams can be controlled more uniformly and may emit radiation more coherently, which makes the generation and preservation of low emittances an area of continuous research as there are ever-present phenomena that generally increase the beam emittance. These include the interaction of charged particles with the fields of other charged particles known as collective effects, "higher-order effects" where transfer matrices are not linear (which are explored in more detail in Appendix A.2), and specific beamline elements may increase the emittances. Others may add correlations between $x, y$, and $z$, such that the beam 
matrix in Equation 2.4 is no longer block diagonal, and the standard definitions of emittances do not apply.

If the beam matrix of Equation 2.4 has correlations, we must come up with a more general invariant to be used as a figure of merit, as the original three emittance definitions are no longer invariant under quadrupoles and other common beamline elements. The so-called "eigen emittances" are the emittances of a matrix that are recovered from a coupled beam matrix, after it has been diagonalized with a diagonalizing matrix [12]. The name is a reference to the underlying method being based on finding the eigenvalues of the beam matrix. When motion is coupled between the $x$ and $y$ plane, for example, the previously defined emittances are variants. However, their product remains an invariant. The product of the three emittances of a $6 \mathrm{D}$ phase space is the general invariant, but the assumptions that we are allowed to make regarding their formalisms depends on coupling within the system.

If the photocathode is immersed in a large axial magnetic field, the motion in $x$ and $y$ is correlated, which breaks the block-anti-diagonality that was utilized for defining the canonical momenta. A set of three quadrupoles downstream that are rotated $45^{\circ}$ to their normal orientation, known as "skew quadrupoles," can remove this correlation. This is due to the torque they provide, as their focusing and defocusing forces are coupled between $x$ and $y$. The newly uncoupled beam will then have a very large ratio between the transverse emittances. For example, if the emittances are on the order of $5 \mu \mathrm{m}$ without the flat-beam transformation, then the emittances after the FBT will have a large ratio, such as 50 and $0.01 \mu \mathrm{m}$. The emittance ratio, $\frac{\varepsilon_{x}}{\varepsilon_{y}}$, may be on the order of 500 , which may be useful for various experiments $[13,14,15]$. For example, a simple case where it is useful is when the emittances (and thus beam sizes) are of unequal importance, such as fitting between 
two dielectric sheets, for which the beam size has tight requirements in one of the transverse planes.

\subsection{Beam Dynamics and Transport}

The methodology and formalism of accelerator physics is, in many ways, similar to that used in traditional radiative optics, wherein the beam's evolution may be described with a series of transfer matrices.

An electron beam, even with a very small emittance, does not remain perfectly collimated over long distances. Due to the natural spread of the beam, it will eventually expand, leading to either beam loss into the pipe walls, or become large enough such that the transfer is no longer linear, resulting in emittance growth. This dilemma necessitates controlled beam transport. At the very least, the beam must be controlled over long distances with quadrupole magnets, which focus the beam in one of the transverse dimensions while defocusing the beam in the other transverse dimension. The behavior is otherwise very similar to optics, with focusing magnets working similar to a convex lens and defocusing magnets like a concave lens.

The other basic beamline element are dipole magnets, that bend the trajectory of the beam in a single dimension. Dipole magnets are used not only for largescale bends, but also as corrector magnets to make slight adjustments to the beam alignment. 


\subsection{Bunch Compression}

For many beams physics applications, it is useful to have longitudinally-compressed bunches. Many factors can contribute positively or detrimentally to the bunch length at beam creation or throughout the experiment. One of the key methods used in compressing a bunch is a chicane bunch compressor. The chicane is comprised of four dipole magnets to induce an energy-dependent path length on the electrons inside the bunch. Higher energy electrons have a shorter path length due to their larger cyclotron radius and the geometry of the chicane (see Figure 2.3 for an example). This is a novel but simple example of the bending radius' dependence on the particle energy, despite all of the electrons traveling at very near the speed of light. This energy-dependent path length property leads to bunch compression if there

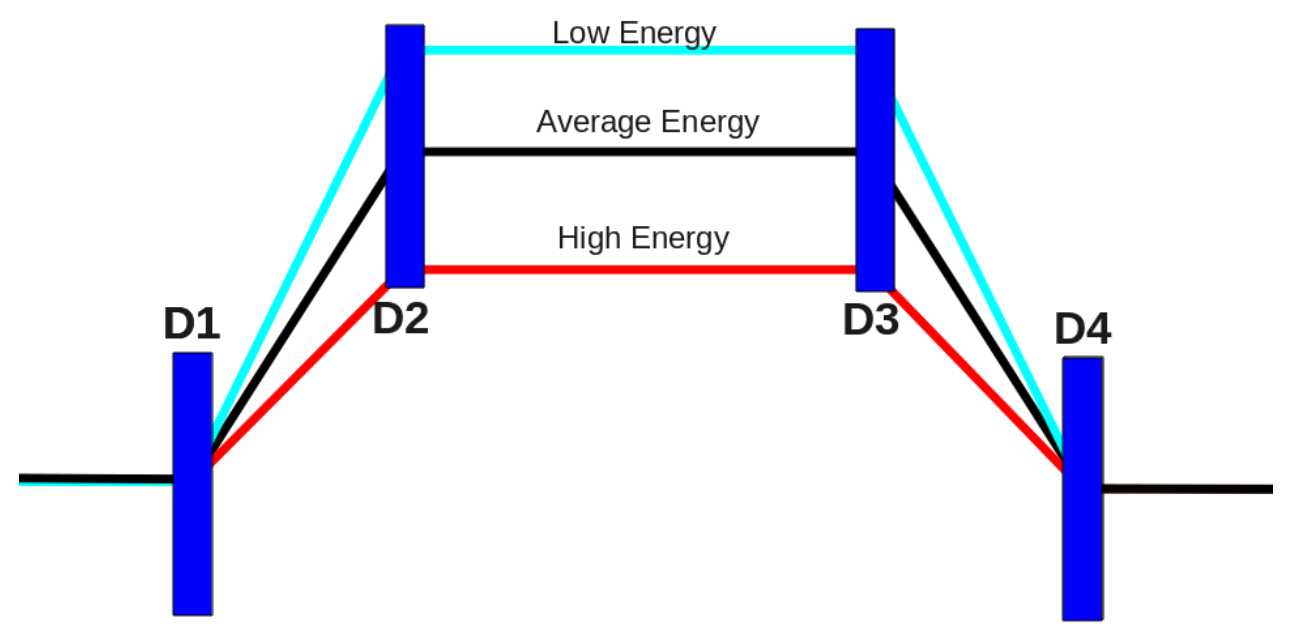

Figure 2.3: An example schematic of a chicane bunch compressor. The blue rectangles D1-D4 are dipole magnets. The colored lines denote paths traced by different energy particles. Higher-energy particles bend less than lower-energy particles, and thus have a shorter path length.

is a systematic energy difference between the head and tail of the bunch, which is achieved when the RF accelerating cavities are operated off-crest, which means that 
the center of the bunch is not receiving maximum acceleration and the accelerating voltage is monotonically increasing or decreasing within the bunch as a function of $z$. The compression inside the bunch compressor is characterized by its $R_{56}$, the term of the 6-dimensional transfer matrix that correlates the initial longitudinal fractional energy deviation at entrance to final longitudinally spatial deviation at the exit.

\subsection{Collective Effects}

There are three main categories of collective effects that are relevant to the status of electron beams. These are space charge (SC) effects where particles directly interact with eachother's fields, synchrotron radiation which is emitted by charged particles as they travel through bends and wakefields in which the fields of a bunch reflect off of structures and materials in the beamline and interact with other particles. A single particle of charge $Q$ has the relativistically distroted fields shown in Equations 1.1.

While the transverse electric field increases with energy, the net transverse force is reduced due by the self-magnetic field, which results in the remaining transverse EM force scaling as $\frac{1}{\gamma^{2}}$. That this scales down with the square of energy is a fundamental aspect of accelerator physics that dictates many aspects of beamline design. Doubling the energy reduces the force by a factor of four, while increasing the energy by a factor of ten reduces it by a factor of one hundred. 
The longitudinal space charge (LSC), the effect of the electric field only in the longitudinal direction, can be written as [16]

$$
\left(\frac{\partial E}{\partial s}\right)_{L S C}=-\frac{2 e}{\gamma^{2}} \frac{\partial \lambda(z)}{\partial z}
$$

where $e$ is the election charge. Due to its dependence on energy, $\propto \frac{1}{\gamma^{2}}$, many experiments hope to take advantage of an increase in energy as a way to reduce the influence of space charge.

When a bunch traverses a dipole magnetic bend, the synchrotron radiation emitted from the tail overtakes the head of the bunch, imparting an energy modulation along the bunch's length. Due to coherent amplification at long wavelengths [17], this coherent synchrotron radiation (CSR) may have a significant impact on the beam's energy. Since this energy modulation occurs within a dispersive location (i.e. a region in which an energy deviation correlates into a spatial spread), it locally "breaks" the achromaticity of the chicane and results in a transverse emittance growth. A one-dimensional model of the corresponding energy redistribution along a bunch traveling on a curved trajectory is detailed in Reference [17]. This model serves as the basis for the CSR models implemented in several of the simulation codes discussed here. The energy redistribution associated with the CSR longitudinal force is

$$
\left(\frac{\partial E}{\partial s}\right)_{C S R}=\frac{2 Q}{R^{2 / 3} 3^{1 / 3}} \int_{-\infty}^{z} \frac{1}{\left(z-z_{w}\right)^{1 / 3}} \frac{\partial \lambda\left(z_{w}\right)}{\partial z_{w}} d z_{w}
$$

where $Q$ is the bunch charge, $R$ is the bend radius, $\lambda(z)$ is the longitudinal projection of the charge distribution, $z$ is the position of the source particle, and $z_{w}$ is that of the witness particle. For a sufficiently long dipole and a bunch with a longitudinal 
charge distribution that has a Gaussian density function, $\lambda(z)=\frac{Q}{\sigma \sqrt{2 \pi}} \exp -\frac{z^{2}}{2 \sigma_{z}^{2}}$, this approximation is characterized by an energy gain near the head of the bunch and an average fractional energy loss of [18]

$$
\Delta \delta=-3.505 \frac{r_{e} Q L_{b}}{e \gamma \sigma_{z}^{4 / 3} R^{2 / 3}}
$$

where $r_{e}$ is the classical electron radius, $Q$ is bunch charge, $e$ is the election charge, and $L_{b}$ is the path length through the dipole. This uncorrelated energy-spread leads to a growth in the transverse emittance due to the change in particle energy mid-chicane, which correlates into a change in $x$ and $x^{\prime}$ at the exit of the chicane.

This semi-analytical approach to CSR assumes the electron bunch as a line charge distribution so that the transverse dimensions of the beam are ignored. The validity of this model is quantified by Derbenev's criterion,

$$
\frac{\sigma_{x}}{\sigma_{z}} 2^{1 / 3}\left[3 \frac{R^{2}}{\sigma_{z}^{2}}\right]^{1 / 3} \ll 1
$$

where $\sigma_{x}$ and $\sigma_{z}$ are the transverse and longitudinal bunch sizes, and $R$ is the bending radius of the dipole. For the beamlines and bunches presented in this dissertation, Derbenev's criterion may be met, not met, or may transition from one regime to another as the bunch passes through a bunch compressor or emittance exchanger. Therefore simulations based on the one-dimensional model should be considered with care.

The characteristic forces of LSC during a drift and CSR inside a dipole bend are shown in Fig. 2.4. Although both of these effects predominantly affect the longitudinal phase spaces, they can couple to the transverse phase space when occurring, for example, in a dispersive section. In addition, the transverse phase space is also 
altered by transverse space charge forces. The key difference between the energy losses is the lack of energy-dependence in the simple 1-D CSR model.

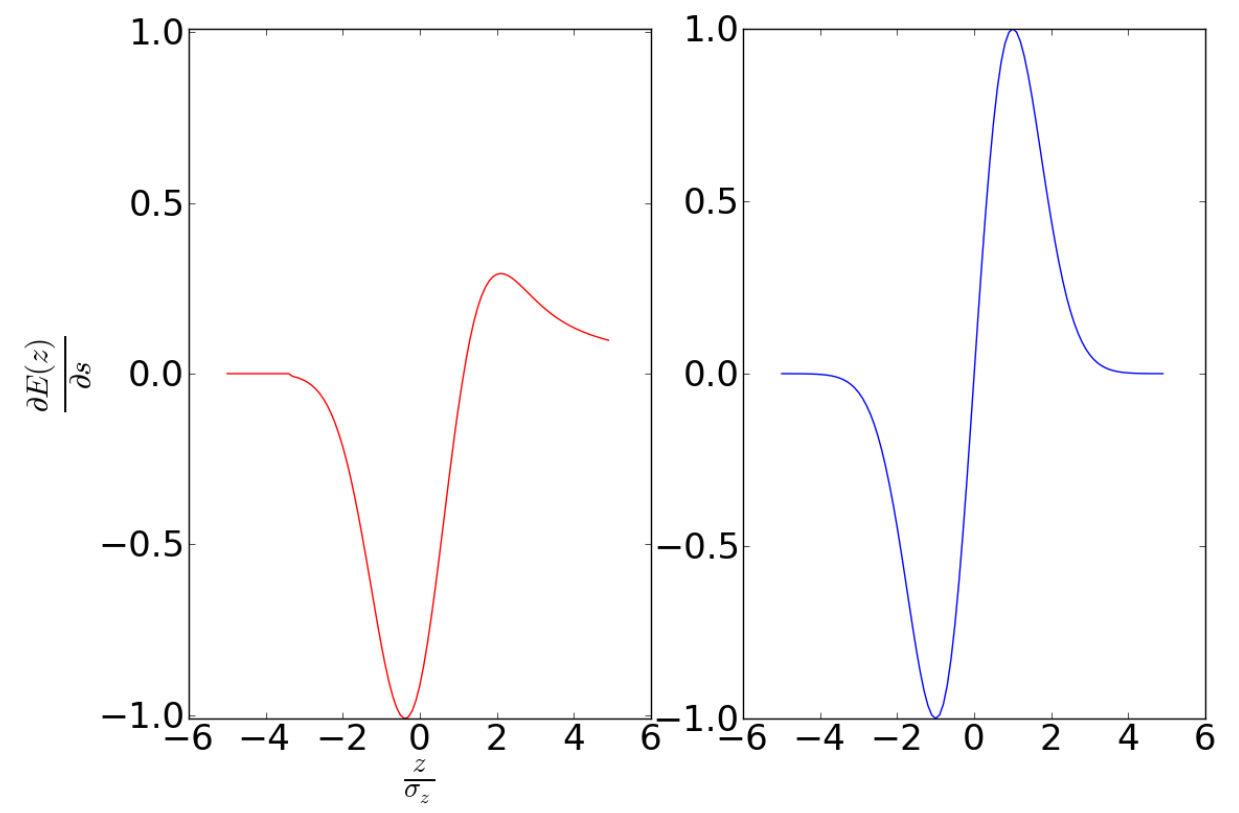

Figure 2.4: Characteristic forces of CSR in a dipole (left) and LSC in a drift (right). To fit on a similar axis, the CSR force is normalized here to $\frac{23 Q}{\sigma_{z} R^{2 / 3} 3^{1 / 3} \sqrt{(2 \pi)}}$, and the $\mathrm{SC}$ force is normalized to $\frac{2 Q e^{-1 / 2}}{\sigma_{z} \gamma^{2}}$.

\subsection{Wakefields}

While CSR is generally considered a tail-to-head effect, wakefields are the opposite, where the field of a particle can interact strongly with those behind it, due to interactions with the material that comprises the beamline. These can interact with both particles in the same bunch, as well as weakly with bunches that occur letter, if the bunches come with a high frequency. For axial-symmetric structures, wakefields may be calculated analytically with many free simulation codes. However, 
axial-asymmetric structures, such as many flanges, bellows, and beam crosses, are difficult to model and require more elaborate particle-in-cell (PIC) codes to model, which oftentimes come with their own difficulties and shortcomings.

\subsection{RF Acceleration}

In linear accelerators, charged particles are accelerated by cavities filled with RF EM fields. The phase of the RF field is timed such that an incoming particle experiences only accelerating fields as it traverses the time-dependent fields of the cavity. Due to the highly relativistic nature of electrons (energies in excess of $100 \mathrm{keV}$ at which point they are travelling at over $90 \%$ of the speed of light), the particle velocity remains roughly the same regardless of how much its momentum and energy increase. This allows the particles to stay in phase with other particles and, perhaps more importantly, in phase with the accelerating cavity even after its energy has increased by a factor of ten or more.

A single charged particle, even at very high energies, has insufficient power and cross-section to be experimentally useful. Instead, many particles are accelerated and transported together as a bunch. The total length of the bunch must be less than half of the RF wavelength that accelerates them, or else some will be decelerated when other particles are accelerated, though the practical limit is much, much smaller, due to the phase-dependent energy gain that arises between particles accelerating on-crest and those accelerating far off-crest. Of particular interest for the NML experiment are 9-cell superconducting RF (SRF) cavities made of niobium, which are then further bundled into groups of eight cavities with a shared cooling and power system, known as a cryomodule. A cryomodule is eight nine-cell cavities 
bound together and sharing a klystron power-supply. The ASTA facility will use a varying number of cryomodules throughout its lifetime, to reach energies ranging from $200 \mathrm{MeV}$ to $900 \mathrm{MeV}$. 


\section{CHAPTER 3 \\ THE ADVANCED SUPERCONDUCTING TEST ACCELERATOR}

The design and construction of the ASTA facility has been undergoing since 2005 [19], and will serve as a testbed for (AAR\&D). In this Chapter, I will present a design overview of ASTA and several studies that aided in the facility's design.

\subsection{The ASTA Facility}

On Fermilab's far North end is the New Muon Laboratory. The facility, seated at the far end of one of the long beamlines shooting North from the main accelerator complex, has had a historic role at Fermilab, but mostly unused for years prior to ASTA's conception. The beamline sits in the basement and related high-bay, fully enclosed by concrete slabs referred to as a "cave". Electronics, power supplies, computing, and the laser laboratory that power and control the experiment are situated outside of the cave, and can be accessed while beam is being created and sent. Due to radiation and high-voltage elements, the cave cannot be accessed while the experiment is running, and numerous safeguards (interlocks) are in place to prevent this.

Due to the length of the ASTA linac (approximately $130 \mathrm{~m}$ ), the NML building is too short for the entire experimental beamline. To accommodate a longer 
beamline, an annex was built across the street to the building's north, along with an underground tunnel connecting the two.

The facility's "cave" is a secure, radiation-shielded zone that cannot be entered during operation due to "interlocks" that turn the accelerator off if the cave gate is opened. For the purposes of this paper, we describe the beamline as several general areas. A diagram of the facility is shown in Figure 3.1. The initial section of ASTA

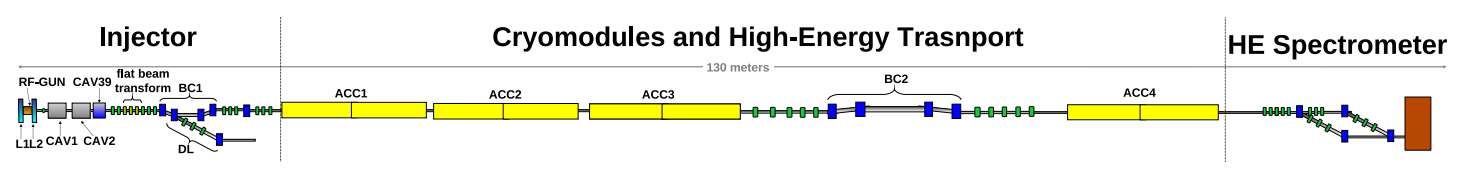

Figure 3.1: Full overview of one possible configuration of the ASTA beamline, divided into three regions, which are the injector, cryomodule and high-energy transport, and high-energy spectrometer. There are experimental areas in both the lowenergy and high-energy areas. Quadrupole magnets are displayed in green, dipole magnets in blue, and accelerating cavities in gray and yellow, For a blow-up of the injector, see Figure 4.1

is known as the injector, which creates and accelerates the beam to high-enough of an energy to mitigate collective effects. It is shown in detail in Figure 4.1. Following the injector is the high-energy line, which contains further acceleration and beam manipulation, before reaching the final diagnostic line and beam dump. Shooting off from the main linac are two experimental areas, one of which is at the same energy as the injector (tens of $\mathrm{MeV}$ ), while the other is placed after acceleration from several superconducting cryomodules (hundreds of $\mathrm{MeV}$ ).

The ASTA injector begins with a photo-emission electron source consisting of a cesium telluride $\left(\mathrm{Cs}_{2} \mathrm{Te}\right)$ photocathode located on the back plate of a $1+1 / 2$ cell radio-frequency $(\mathrm{RF})$ cavity operating at $1.3 \mathrm{GHz}[20]$ (commonly referred to as the RF "gun"). The cathode is illuminated with a 3-ps ultraviolet laser pulse with uniform radial distribution and a Gaussian temporal profile. The optimum laser RMS spot size for minimal emittance depends on the bunch charge and ranges from 
$80 \mu \mathrm{m}$ (for $20 \mathrm{pC}$ ) to $1.3 \mathrm{~mm}$ (for $3.2 \mathrm{nC}$ ). The $\mathrm{RF}$ gun is surrounded by two solenoidal lenses that control the beam's transverse size and emittance, and a third that may be used to aid in flat-beam transformations [21]. The gun system emits the electrons at a typical beam energy of $\sim 5 \mathrm{MeV}$. A photograph of the gun system is shown in Figure 3.2.

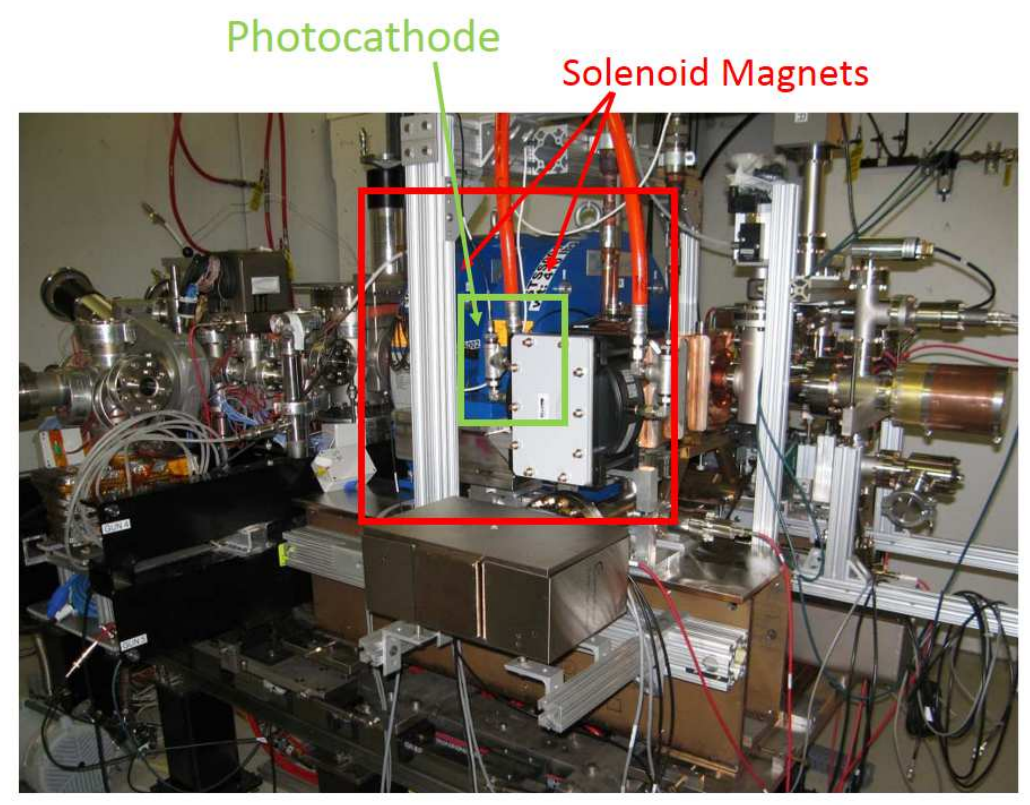

Figure 3.2: Picture of the ASTA electron source gun from Reference [?].

Immediately downstream of the gun are two accelerating cavities, CAV1 and CAV2. Together, these accelerate the beam from $5 \mathrm{MeV}$ to $50 \mathrm{MeV}$. In addition, CAV2 imparts the longitudinal energy correlation (energy chirp) that allows for bunch compression. A third SRF cavity (CAV39) operating at $3.9 \mathrm{GHz}$ will eventually be incorporated to correct for nonlinear longitudinal phase space distortions [22, 23, 24], as discussed in Chapter 2. It will not be included in the beamline at the time of initial beam conditioning due to restrictions on the $3.9 \mathrm{GHz}$ RF system, which places limits on how much compression can be achieved in the bunch 
compressor for the first few months or year of operation. A bare cavity and a cryomodule that contains a cavity are shown in Figure 3.3.
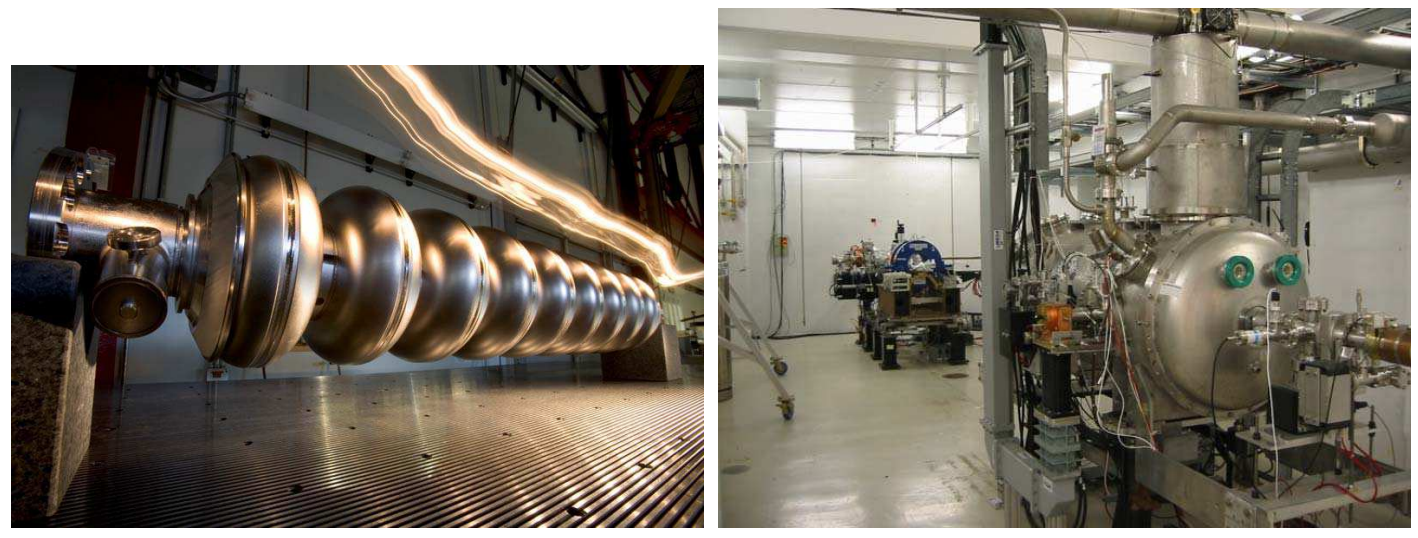

Figure 3.3: (left) A picture of a bare niobium nine-cell cavity, before its insertion into a shell for cryogenics, and (right) the cryo shell that contains one such cavity as placed in ASTA, from Reference [?].

Downstream of the booster and linearizer cavities (CAV1, CAV2, and CAV39), but before the bunch compressor, are a set of seven quadrupole magnets. Four of these are placed to control the transverse beam dynamics, while the other three are "skew" quadrupole magnets used in the round-to-flat-beam transformation (RFBT), which produce coupled forces between the horizontal and vertical dimensions. In particular this can remove the correlation imparted by L2, resulting in a beam with a large ratio of transverse emittances.

The longitudinal phase space behavior of the beam after the gun, RFBT, and bunch compressor will eventually be explored with a spectrometer, which is a a system of a transverse deflecting cavity followed by a bending magnet. This functions as a way to view the longitudinal phase space pulse-by-pulse [25]. This will not be implemented during beam commissioning, but will instead be added in a later phase of ASTA operation due to its use of a $3.9 \mathrm{GHz} \mathrm{RF}$ system that is not 
yet installed. For a more detailed overview of the design and performance of the longitudinal spectrometer, see Chapter 5.

Whereas CAV1 and CAV2 were each single "9-cell cavities" [?], a cryomodule is eight such cavities combined into a single unit with shared power supplies cooling systems. ASTA will serve as a test facility for cryomodules that will be implemented in future accelerator facilities.

Downstream of the injector is the high-energy beamline. This consists of a variable number of superconducting cryomodules, beam transport, and a high-energy spectrometer. Most of these components are analogous to components in the injector, but with some key differences and different functionality.

Due to the varying number of cryomodules that will be included at ASTA, the beamline downstream of them must be flexible as its requirements change with the number of cryomodules to be used, or potential user experiments. A flexible FODO lattice was determined to be a viable solution, and a detailed design study is presented later in this chapter.

The HE beamline consists of a dispersive section followed by a beam transport line to the high-power beam dump. The purpose of this dispersive section is to measure the beam energy and energy spread. The dispersive section is composed of a dogleg with two dipoles of $\pm 15 \mathrm{deg}$, shown as D600 and D604 in Figure 3.4.

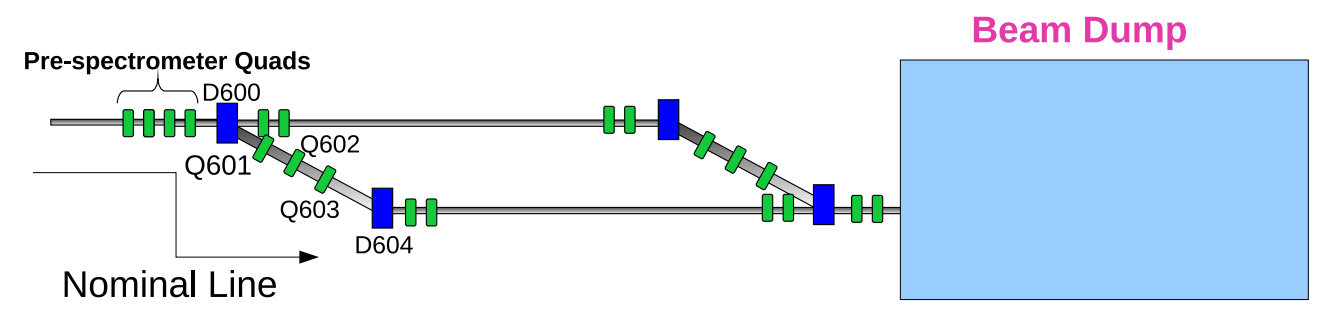

Figure 3.4: Schematic diagram of the two spectrometers. 
In order for the beam's energy to be dissipated safely at the end of its transport, it must be diverted into a beam dump, which is a large block of concrete and steel [?]. ASTA contains two beam dumps. One is at low-energy, at the end of the longitudinal spectrometer system prior to CM1, which is designed for $50 \mathrm{MeV}$ beams. The other beam dump is downstream of the high-energy beamline, to absorb beam energies of hundreds of $\mathrm{MeV}$.

\subsection{Planned Advanced Accelerator Research and Development}

ASTA has been in the planning, construction, and commissioning stages since around 2007 [19]. As a result of the facility's intersection between the National, International Llinear Collider (ILC), and Fermilab physics programs, its purpose is multi-faceted. Even at the time of this writing, in 2013, the plans and funding are in flux. As the original plan for ASTA was to function as an ILC test accelerator, the cryomodules and their configurations have been a significant feature. Over the years, the target number of cryomodules, types, and energies have changed. Overtime, plans have been floated for anywhere between one-to-six cryomodules, with a group of three representing one ILC "unit", that may share cooling and power systems. In addition, a second bunch compressor (BC2) similar to BC1 may be included downstream of the cryomodule, which would be used to impart energy chirp to the beam in the same way that CAV2 is used for the low-energy bunch compressor.

The implementation of the overall facility, at the time of this writing, is as follows [?]. In Phase I, only a single cryomodule will be in operation, allowing beam 
energies up to $\sim 300 \mathrm{MeV}$. It is during this phase that the Integrable Optics Test Accelerator (IOTA) will be implemented [?], for the testing of non-linear optics systems. In Phase II, a second and third cryomodule will be in place, allowing beam energies up to $800 \mathrm{MeV}$. Later phases may allow for a fourth cryomodule [?], or a second bunch compressor. The implementation of user experiments will vary per phase.

Due to the variable number of cryomodules and the fixed location of the highenergy beam dump, a transport solution was needed that could transport the beam long distances (around 100 meters at its maximum), use few quadrupole magnets, and be scaled down as the beamline becomes filled in with cryomodules or other components. A study including several such designs is presented in the following section.

\subsection{First Beam Design of the High-Energy Transport Line}

As part of my work, I designed a flexible beamline between the cryomodules and the high-energy spectrometer [26]. The design was made with several key constraints. The beamline used to transport the beam between the cryomodule and the HE beamline should be simple, easily reconfigurable (e.g., to accommodate the 2nd and 3rd cryomodules when they are installed), and able to support the first set of planned experiments. It should also include provisions for beam diagnostics, especially transverse emittance measurements. The design must incorporate a limited number of high-energy (HE) quadrupole magnets. A FODO lattice was chosen as it meets our requirements, can be assembled from the available beamline components, 
and is easily scalable for reconfigurations (e.g., when other accelerating modules become available).

Between the exit of the first cryomodule and the first dipole of the HE beamline, the lattice incorporates a total of twelve quadrupole magnets: $(i)$ four to match the beam C-S parameters to the FODO lattice, $(i i)$ four to actually comprise the FODO lattice, and (iii) four to match the beam to the desired C-S parameters at the spectrometer entrance. A schematic diagram of the FODO lattice appears in Figure 3.5.

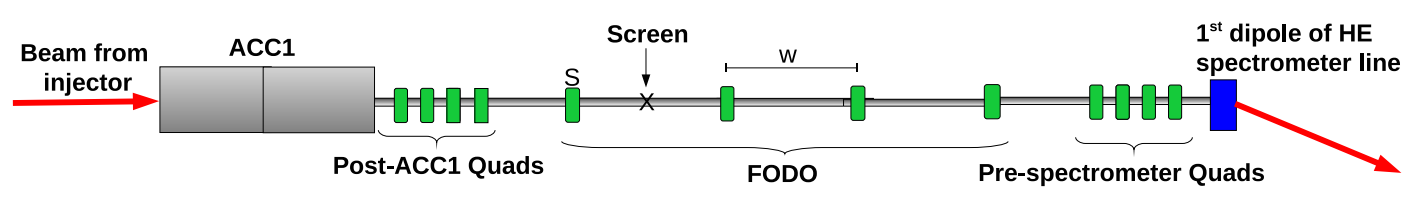

Figure 3.5: Schematic of the FODO lattice, showing the four quadrupole magnets after the cryomodule, the four quadrupole magnets that comprise the FODO lattice itself, and the four quadrupole magnets that match into the spectrometer. The "S" and "X" symbols respectively refer to the quadrupole magnet used for the quadrupole magnet scanned and the profile monitor used for the transverse emittance measurements.

Given the available length for the FODO lattice, the small number of allotted quadrupole magnets places constraints on the betatron function. The design study was performed using the simulation code ELEGANT; the code is discussed in more detail in Appendix C. The minimum and maximum values of the betatron functions in the FODO cell can be parametrized as a function of the betatron phase advance per cell $\Psi$ as [27]

$$
\begin{aligned}
& \beta_{\text {max }}=\frac{w}{\sin \frac{\Psi}{2}} \sqrt{\frac{1+\sin \frac{\Psi}{2}}{1-\sin \frac{\Psi}{2}}}, \text { and } \\
& \beta_{\text {min }}=\frac{w}{\sin \frac{\Psi}{2}} \sqrt{\frac{1-\sin \frac{\Psi}{2}}{1+\sin \frac{\Psi}{2}}},
\end{aligned}
$$


Table 3.1: Parameters concerning the FODO lattice.

\begin{tabular}{lccc}
\hline \hline Parameter & Symbol & Value & Unit \\
\hline Bunch Charge & $\mathrm{Q}$ & 3.2 & $\mathrm{nC}$ \\
Effective Quadrupole Magnet Length & $\mathrm{L}$ & 0.417 & $\mathrm{~m}$ \\
Quadrupole Magnet Separation (center-to-center) & $w$ & 16.4 & $\mathrm{~m}$ \\
Phase Advance & $\Psi$ & 63 & $\mathrm{deg}$ \\
Screen Position from Quadrupole Magnet & $D$ & 8.4 & $\mathrm{~m}$ \\
min betatron function & $\beta_{\min }$ & 20 & $\mathrm{~m}$ \\
max betatron function & $\beta_{\max }$ & 60 & $\mathrm{~m}$ \\
$\max$ spot size for $\epsilon_{n}=0.1 \mu \mathrm{m}$ at $300 \mathrm{MeV}$ & $\sigma_{\max }$ & 0.10 & $\mathrm{~mm}$ \\
$\max$ spot size for $\epsilon_{n}=0.1 \mu \mathrm{m}$ at $40 \mathrm{MeV}$ & $\sigma_{\max }$ & 0.28 & $\mathrm{~mm}$ \\
$\max$ spot size for $\epsilon_{n}=1 \mu \mathrm{m}$ at $300 \mathrm{MeV}$ & $\sigma_{\max }$ & 0.32 & $\mathrm{~mm}$ \\
$\max$ spot size for $\epsilon_{n}=1 \mu \mathrm{m}$ at $40 \mathrm{MeV}$ & $\sigma_{\max }$ & 0.88 & $\mathrm{~mm}$ \\
$\max$ spot size for $\epsilon_{n}=10 \mu \mathrm{m}$ at $300 \mathrm{MeV}$ & $\sigma_{\max }$ & 1.01 & $\mathrm{~mm}$ \\
$\max$ spot size for $\epsilon_{n}=10 \mu \mathrm{m}$ at $40 \mathrm{MeV}$ & $\sigma_{\max }$ & 2.77 & $\mathrm{~mm}$ \\
$\max$ spot size for $\epsilon_{n}=50 \mu \mathrm{m}$ at $300 \mathrm{MeV}$ & $\sigma_{\max }$ & 2.26 & $\mathrm{~mm}$ \\
$\max$ spot size for $\epsilon_{n}=50 \mu \mathrm{m}$ at $40 \mathrm{MeV}$ & $\sigma_{\max }$ & 6.19 & $\mathrm{~mm}$ \\
\hline \hline
\end{tabular}

where $w$ is the half-period of the lattice. Tab. 3.1 shows the selected values for FODO lattice, and the maximum spot size for several normalized emittances and energies, and Figure 3.6 presents $\beta_{\max }$ and $\beta_{\min }$ as functions of $\Psi$ and $w$.

The transverse beam diagnostics place additional upper and lower boundaries on the betatron functions. The total beam size $\sim 8 \sigma$ should fit within the field of view of the imaging system used to measure the beam size. In addition, the beam size should be at least twice as large as the resolution $\sigma_{r e s}$ of the beam size measurement method. These two constraints place limits on the lattice functions at the screen $\beta_{\text {screen }}$

$$
\frac{4 \gamma \sigma_{r e s}^{2}}{\epsilon_{n}} \leq \beta_{\text {screen }} \leq \frac{d_{F o V} \gamma}{64 \epsilon_{n}}
$$



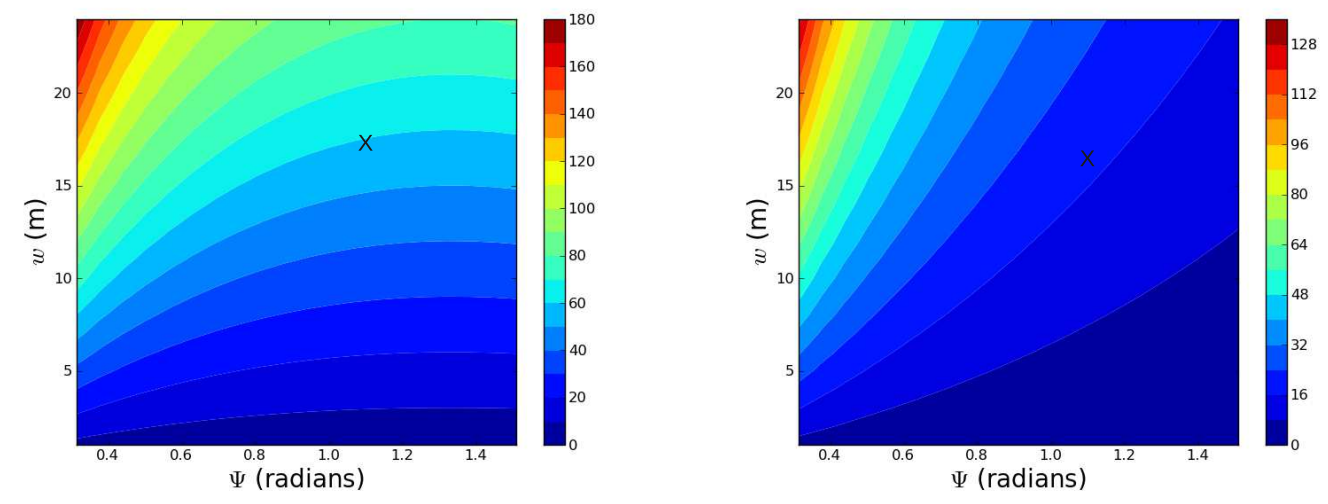

Figure 3.6: Maximum (left) and minimum (right) values of the betatron function in the FODO lattice as functions of the betatron phase advance $\Psi$ and the center-tocenter distance between quadrupole magnets, $w$. The "X" symbol at $(\Psi, w) \simeq(1.1$, $17 \mathrm{~m}$ ) indicates the operating point of the FODO lattice selected for the first beam configuration.

where $d_{F o V}$ is the field of view of the imaging system, $\sigma_{\text {res }}$ is the resolution of the measurement, $\gamma$ is the beam's relativistic mass factor, and $\epsilon_{n}$ is the transverse normalized emittance; these constraints are shown in Figure 3.7.

The C-S parameters entering the cryomodule-to-HE beamline depend on the cryomodule operating parameters as significant ponderomotive focusing occurs in the cryomodule. The transverse ponderomotive force [28] is given by

$$
F_{r}=\frac{\chi_{i} \phi\left(e E_{0}\right)^{2} r}{8 \gamma m_{0} c^{2}} \propto r
$$



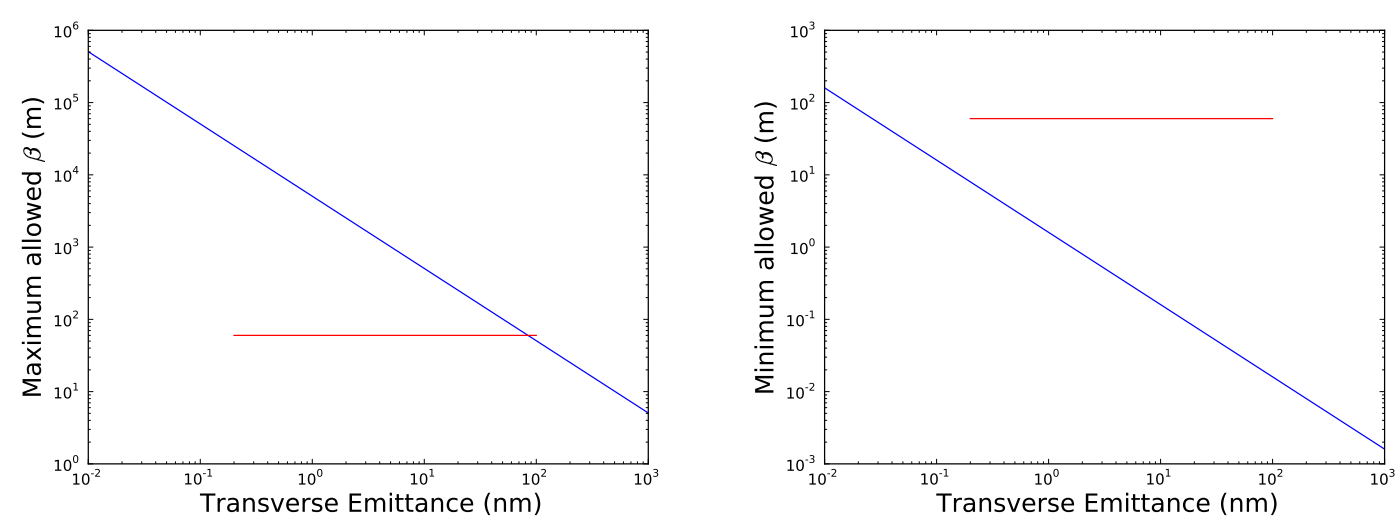

Figure 3.7: Maximum (left) and minimum (right) acceptable values for the betatron function (blue traces) at the transverse profile monitor as functions of the transverse geometric (unnormalized) emittance, $\epsilon$. The red line denotes the operating range of the FODO channel specified in Tab. 3.1. The maximum (resp. minimum) value of the betatron function is determined by the size (resp. the resolution) of the transverse profile monitor used for beam size measurement.

where $\gamma m_{0} c^{2}$ is the total particle energy ( $\gamma$ is the Lorentz factor), $\phi$ is the particle's phase with respect to the maximum accelerating phase, $r$ is the distance from the cryomodule axis, $e$ is the electric charge, $E_{0}$ is the average accelerating field, and $\chi_{i}$ describes the harmonic content of the spatial field distribution. We take $\chi_{i} \sim 1$ for a standing wave structure which is significant in the first accelerating cavities of the cryomodule as $E_{0} \approx 32 \mathrm{MV} / \mathrm{m}$ and $\gamma m_{0} c^{2} \approx 40 \mathrm{MeV}$. Therefore we should expect the C-S parameter to be significantly affected by the cryomodule parameters, e.g., its operating phase. Though we expect to never operate more than $30^{\circ}$ off-crest, we present more extreme cases in this section to illustrate the robustness of our design.

A possible solution involves focusing the beam at the cryomodule entrance (i.e., $\alpha>0)$ and entering the cryomodule with a small beam size $(\beta<20 \mathrm{~m})$. Such a beam would lower the value of $r$ in Eq. 3.4 and thereby reduce the sensitivity to the ponderomotive force as the particles are transported through the cryomodule. A divergent beam will therefore exit the cryomodule. This solution has the overall 
benefit of reducing the transverse focusing effect of the RF fields, which allows for more consistent behavior across varying peak fields and operating phases. Figure 3.8 shows a comparison of the evolution of the betatron function inside the cryomodule for two different initial values of $\beta$. The smaller beam size offers more uniformity of roughly half the phase-dependent variation in $\beta$ and one-third less variation in $\alpha$, which reduces the amount of re-matching that must be done to match into the FODO channel. Transmission through the cryomodule and the C-S parameters at its exit as functions of the C-S parameters at its entrance for various operating phases are shown in Figs. 3.9-3.12, using an effective normalized emittance $\epsilon_{n}$ of $100 \mu \mathrm{m}$. The chosen large value for the emittance gives a safe-side estimate and accounts for possible bunch-to-bunch jitter and beam misalignment.
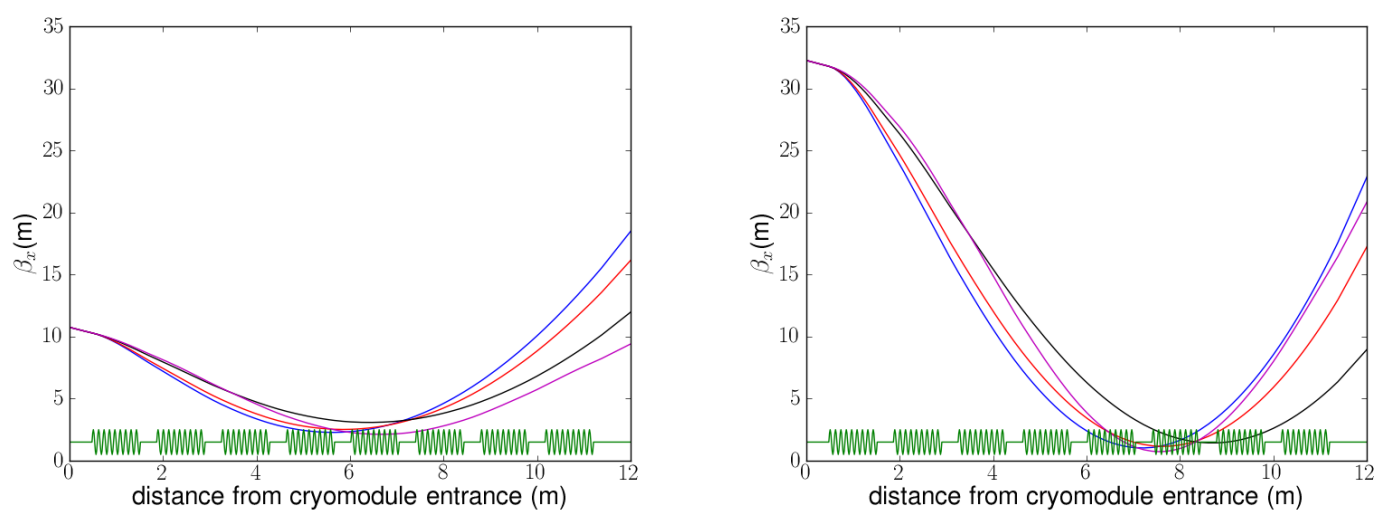

Figure 3.8: Lattice functions inside the cryomodule for different operating phases [0 "on-crest" (red), 30 (blue), 60 (black) and 90 (magenta)] off-crest for $(\alpha, \beta)=$ $(0.5,10.75 \mathrm{~m})$ (left) and $(\alpha, \beta)=(0.5,32.25 \mathrm{~m})$ (right). The green line indicates the position of the eight ILC cavities (each cell shown as a wiggle) composing the cryomodule. A higher initial betatron function leads to beams with larger and more divergent betatron function values downstream of ACC1. The final correlation is $\alpha \in[-2.5,-1.0]$ (left) while $\alpha \in[-4.6,-2.4]$ for the larger initial betatron-function value (right). 


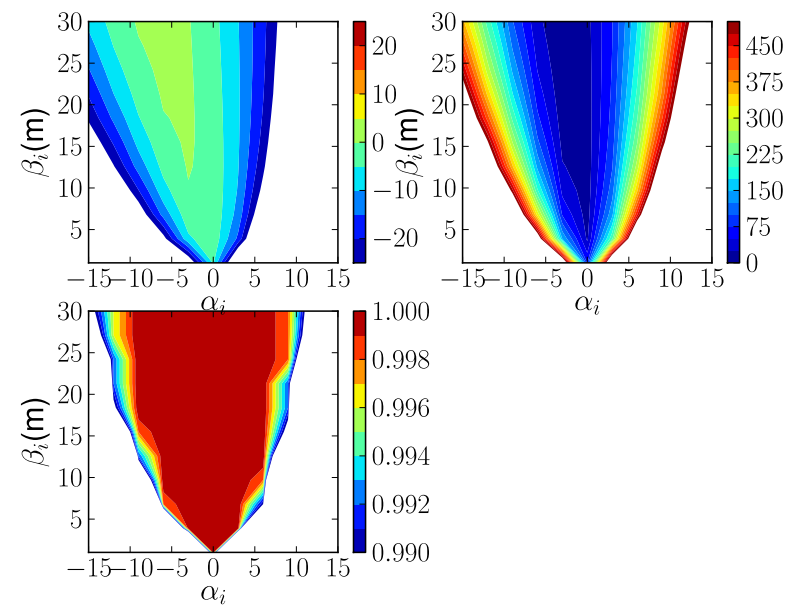

Figure 3.9: Contour plots for $\alpha_{f}$ (upper left), $\beta_{f}$ (upper right) and transmission through the cryomodule (lower plot) at the cryomodule exit for the nominal initial C-S parameters $(\alpha, \beta)=(0.5,11 \mathrm{~m})$, with ACC1 operated on-crest $\left(\phi=0^{\circ}\right)$. The units for the $\beta_{f}$, and the transmission contour plots are respectively meters and percents.

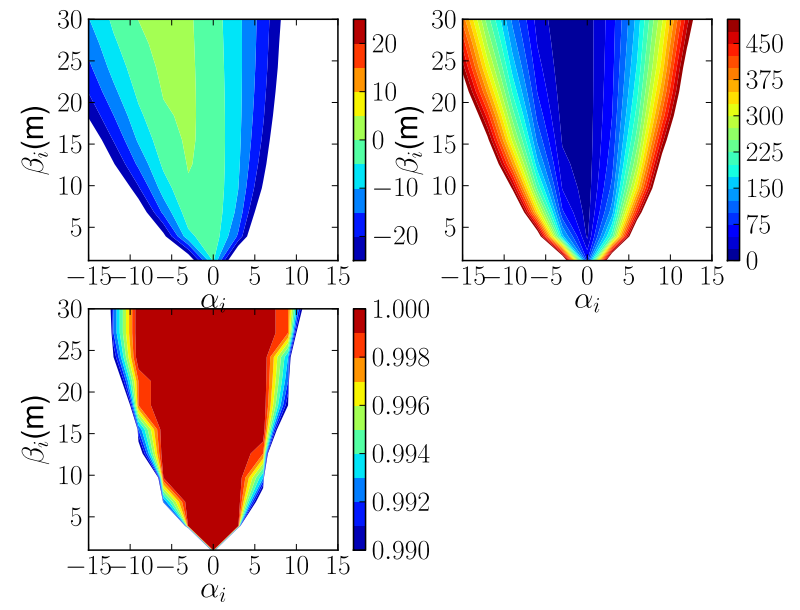

Figure 3.10: Contour plots for $\alpha_{f}$ (upper left), $\beta_{f}$ (upper right) and transmission through the cryomodule (lower plot) at the cryomodule exit for the nominal initial C-S parameters $(\alpha, \beta)=(0.5,11 \mathrm{~m})$, with ACC1 operated at $\phi=30^{\circ}$ off-crest. The units for the $\beta_{f}$, and the transmission contour plots are respectively meters and percents. 


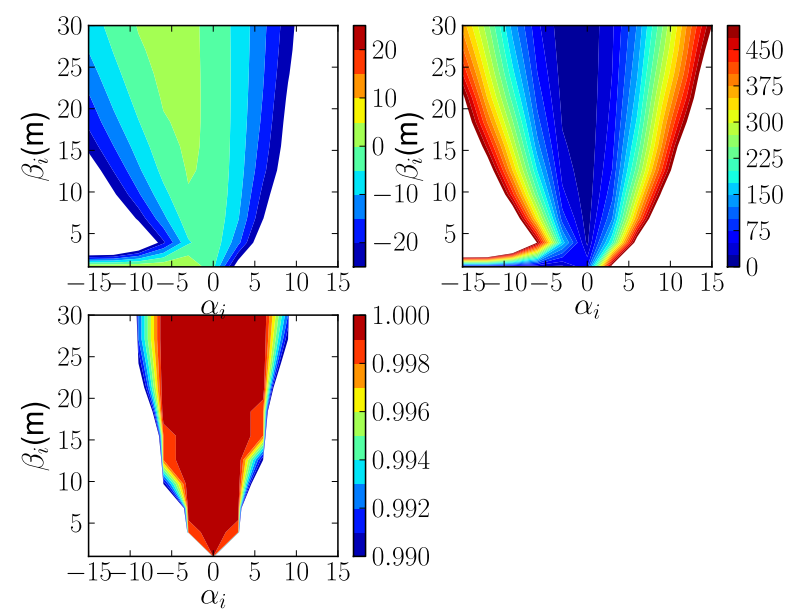

Figure 3.11: Contour plots for $\alpha_{f}$ (upper left), $\beta_{f}$ (upper right) and transmission through the cryomodule (lower plot) at the cryomodule exit for the nominal initial C-S parameters $(\alpha, \beta)=(0.5,11 \mathrm{~m})$, with ACC1 operated $\phi=60^{\circ}$ off-crest. The units for the $\beta_{f}$, and the transmission contour plots are respectively meters and percents.

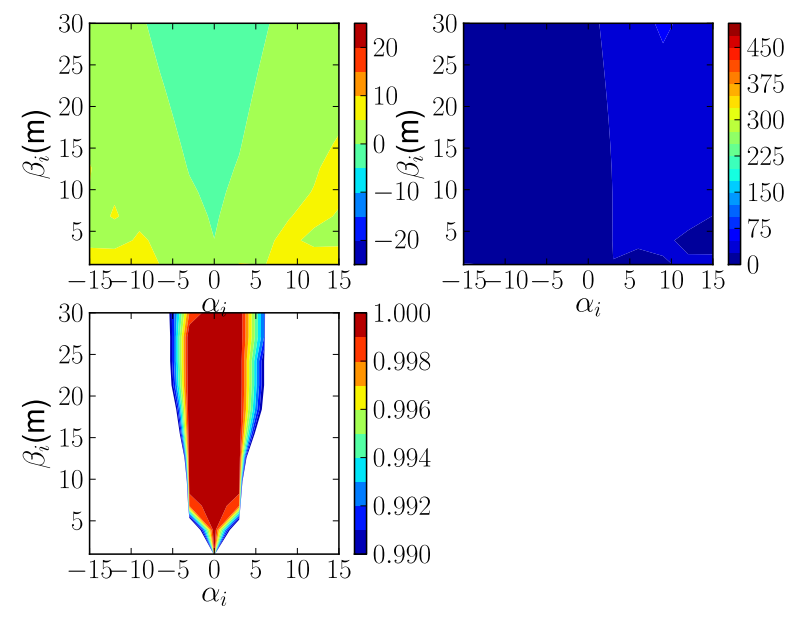

Figure 3.12: Contour plots for $\alpha_{f}$ (upper left), $\beta_{f}$ (upper right) and transmission through the cryomodule (lower plot) at the cryomodule exit for the nominal initial C-S parameters $(\alpha, \beta)=(0.5,11 \mathrm{~m})$, with $\mathrm{ACC} 1$ operated $\phi=90^{\circ}$ off-crest. The units for the $\beta_{f}$, and the transmission contour plots are respectively meters and percents. 


\subsection{HE Spectrometer and Dump}

Downstream of the cryomodules and transport line is a dispersive section followed by a beam transport line to the high-power beam dump, which is used to measure the beam energy and energy spread. It is shown in Figure 3.4.

The dipoles are rectangular dipoles tilted by 7.5 deg to relax the requirement on field uniformity over a smaller transverse region (the beam entrance and exit angles are $+7.5 \mathrm{deg}$ ). Two quadrupole magnets (Q601 and Q603) inserted between the dipoles provide ensure that the horizontal dispersion function and its slope $\left(\eta_{x}, \eta_{x}^{\prime}\right)$ vanish downstream of the second dipole (D604). Such settings provide a dispersion function with two extrema within the dogleg with values of $\pm 0.6 \mathrm{~m}$ (see Figure 3.13).
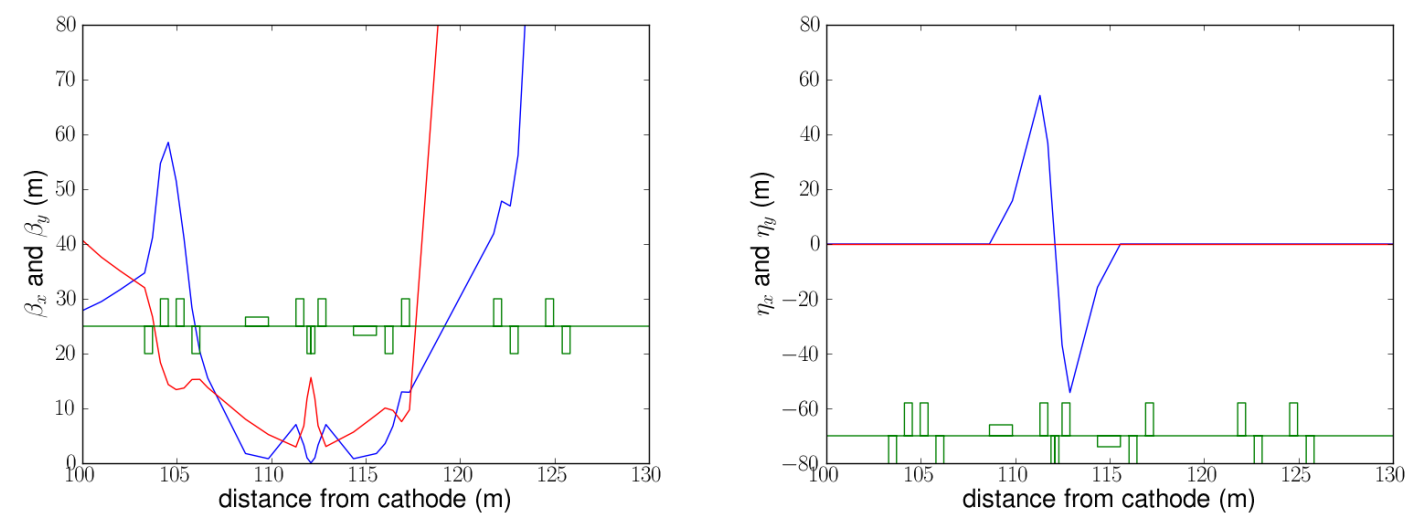

Figure 3.13: Evolution of the betatron functions $\beta_{x}$ (red) and $\beta_{y}$ (blue) [left] and dispersion function $\eta_{x}$ (red) and $\eta_{y}$ (blue) [right] along the HE spectrometer line.

A third quadrupole (Q602) located at the vanishing dispersion point within the dogleg enables further control of the betatron functions. Finally, six quadrupole magnets located downstream of the dogleg are used to defocus the beam on the 
dump window at $(\mathrm{z}=131 \mathrm{~m})$. In a dispersive section the RMS beam size, at location $s$, is given by

$$
\sigma_{x}=\sqrt{\beta_{x}(s) \epsilon_{x}+\left(\eta_{x}(s) \sigma_{\delta}\right)^{2}} \approx \eta_{x} \sigma_{\delta}
$$

The right-hand-side approximation assumes that $\left(\eta_{x} \sigma_{\delta}\right)^{2} \gg \beta_{x} \epsilon_{x}$. Considering a $5 \mathrm{~cm}$ diameter beam pipe and given that $4 \sigma$ of the beam should be contained within the beam pipe's aperture, we find a limit of $\sigma_{x} \leq 1.25 \mathrm{~cm}$. The high dispersion point places a limit on the FES of $\sigma_{\delta} \leq 2 \%$ (RMS). As a comparison, a cryomodule with accelerating voltage $V_{R F}$ operated with an off-crest phase $\phi$ results in a RMS correlated energy spread $\sigma_{\delta_{f}}$

$$
\begin{aligned}
\sigma_{\delta_{f}} & =\sqrt{A+B+C}, \\
A & =\left(\frac{E_{i}}{E_{i}+e V_{R F} \cos \phi}\right)^{2} \sigma_{\delta i}^{2}, \\
B & =\left(\frac{e V}{E_{i}+e V \cos \phi}\right)^{2}\left(k^{2} \sigma_{z, i}^{2}\right) \sin (\phi)^{2}, \\
C & =\frac{3 k^{4}}{4} \sigma_{z, i}^{4} \cos (\phi)^{2} .
\end{aligned}
$$

where $\sigma_{\delta i}$ is the initial FES, $k \equiv \frac{2 \pi}{\lambda}$ is the wave vector modulus, $\sigma_{z, i}$ is the initial RMS bunch length, $E_{i}$ is the initial energy, $e$ is the electron charge, and $\phi$ is the accelerating phase with 0 corresponding to on-crest. Final energy spread is shown as a function of operating phase for various bunch lengths in Figure 3.14. 


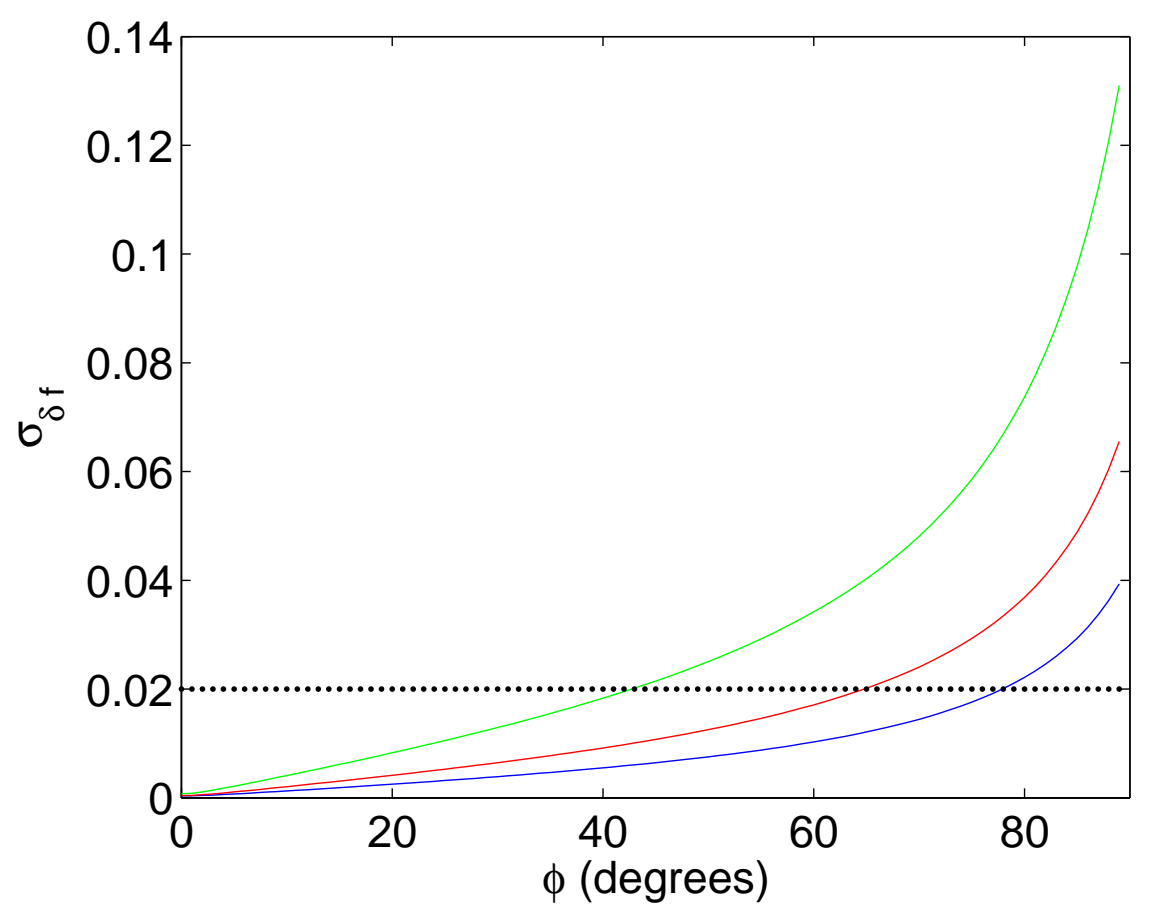

Figure 3.14: Final relative fractional energy spread $\sigma_{\delta f}$ as a function of off-crest phase $\phi$, for $\sigma_{z, i}=300$ (blue), 500 (red) and 1000 (green) $\mu \mathrm{m}$, with $E_{i}=40 \mathrm{MeV}$, $V_{R F}=210 \mathrm{MV}$, and $\sigma_{\delta, i}=0.22 \%$. The black dotted line shows the upper-limit of $2 \%$ that the dispersive section places on our energy spread.

\subsection{Performance Studies}

The performance of the designed lattice has been checked for several operating phases of the cryomodules. Two operating scenarios have been explored: One where the upstream telescope is used to rematch the beam into the FODO lattice as the cryomodule phase is varied, and another where the strength of the quadrupoles is adjusted only to account for the varying beam energy. The results shown in Figure 3.15-3.17, demonstrating the ability of the proposed beamline to accommodate ACC1 phase over the full range of anticipated phases (conservatively up to $60^{\circ}$ offcrest, though we expect to never run more than $30^{\circ}$ off-crest). This FODO design 
allows for intact transport of the beam for a variety of tested cryomodule phases, of at least $30^{\circ}$ off-crest with or without the re-matching of the four quadrupole magnets downstream of the cryomodule exit. The selected spacing of the quadrupole magnets sets minimum and maximum beta functions that can be easily matched for the tested range of phases. For optimal behavior, the quadrupoles should be re-matched when the phase of the cryomodule is changed to account for the different RF-focusing, but even at $30^{\circ}$ off-crest the deviation from the proper FODO behavior is small (Figure 3.16) and the spot size at the beam dump is within 10\% of the on-crest behavior. Since the rematching would require an iterative procedure involving several emittance measurements, it is more appealing from the operational standpoint not to have to rematch.

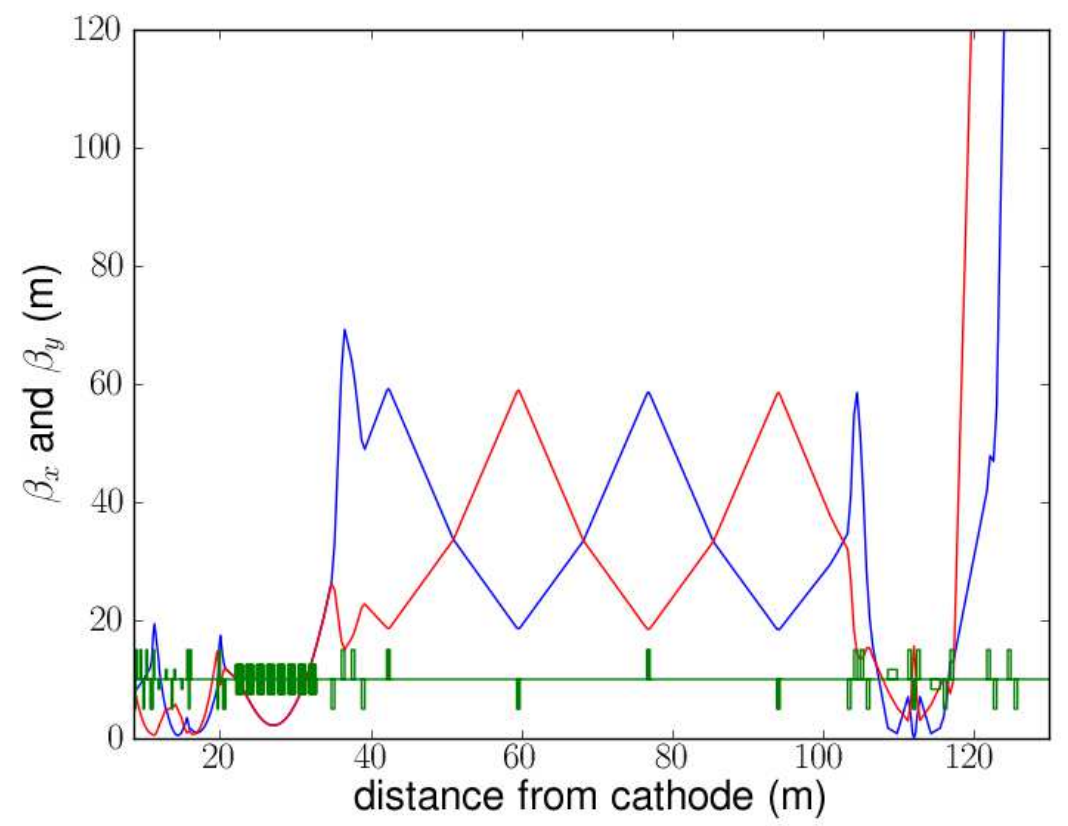

Figure 3.15: Betatron functions along the beamline, with ACC1 operated on-crest $\left(\phi=0^{\circ}\right)$. 

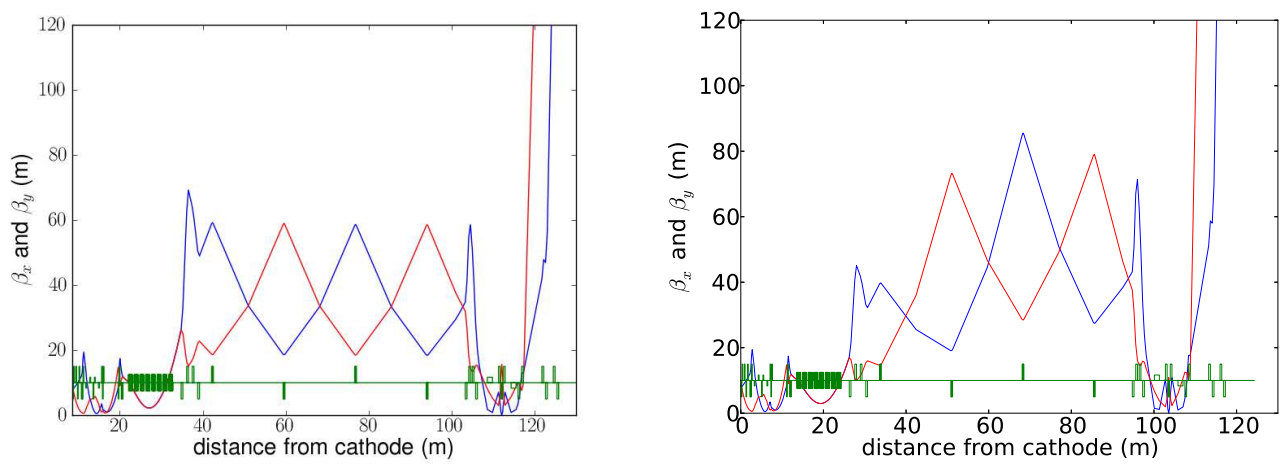

Figure 3.16: Betatron functions along the beamline, with ACC1 operated thirtydegrees off-crest $\left(\phi=30^{\circ}\right)$, with (left) and without (right) rematching.
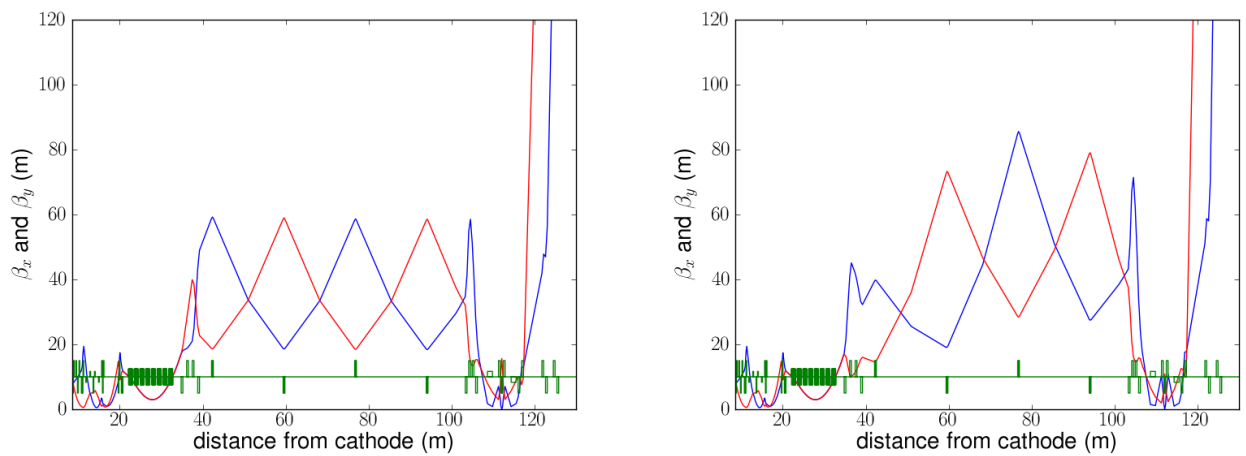

Figure 3.17: Betatron functions along the beamline, with ACC1 operated sixtydegrees off-crest $\left(\phi=60^{\circ}\right)$, with (left) and without (right) rematching.

\subsection{Transverse emittance measurements}

Downstream of ACC1, the beam's transverse emittances are foreseen to be measured using the quadrupole magnet scan method [29]: a quadrupole magnet's field will be scanned and the beam size recorded on a downstream optical transition radiation (OTR) viewer or scintillator screen [30]; see Figure 3.5. The OTR beam profiling system, to be constructed by RadiaBeam Technologies to Fermilab's specifications, is characterized to have a $14 \mu \mathrm{m}$ and $7 \mu \mathrm{m}$ resolutions for a field of view 
of 15 and $5 \mathrm{~mm}$ respectively [31], though this design is still tentative. Based on the position of the screen $8.63 \mathrm{~m}$ after the first quadrupole magnet, we use the four quadrupole magnets immediately downstream of the cryomodule to focus to C-S parameters at "S" such that $\frac{\alpha_{x, y}}{\beta_{x, y}}=\frac{1}{D}$ where $D$ is the drift length from the quadrupole magnet's center to the downstream screen. This condition ensures that the beam size on the screen is the smallest at the middle of the scan, i.e., when the quadrupole magnet's field strength $k_{1}$ is set to 0 , and reduces the effect of error.

The quadrupole magnet scan method was simulated in ELEGANT by scanning the strength of the first quadrupole magnet of the FODO lattice and recording the RMS beam size at the location of the profiling system. From the spot size and the strengths of the quadrupole magnets, we are able to recover the beam emittance at the exit of the cryomodule using a least-squares fit method.

To account for resolution effects, we quadratically add the resolution to the simulated beam sizes.

$$
\sigma=\sqrt{\sigma_{\text {sim }}^{2}+\sigma_{r e s}^{2}}
$$

We simulate possible statistical error from many potential sources by adding a random fluctuation to the beam size

$$
\sigma_{\text {meas }}=\sigma(1+\Delta \zeta)
$$

where $\Delta$ is the relative amplitude of the statistical uncertainty and $\zeta$ is a random variable following a Gaussian distribution with variance of 1.

As a first case we simply took the nominal beam line settings and scanned the quadrupole magnet's strength. Such a configuration leads to asymmetric evolution 
of the horizontal and vertical beam sizes; see Figure 3.18 (right). On the other hand, when the four preceding quadrupole magnets are tuned such that the condition $\frac{\alpha}{\beta}=\frac{1}{D}$ is satisfied, one obtains a symmetric evolution of the beam sizes which is optimal for achieving similar resolution in the horizontal and vertical emittance measurements.
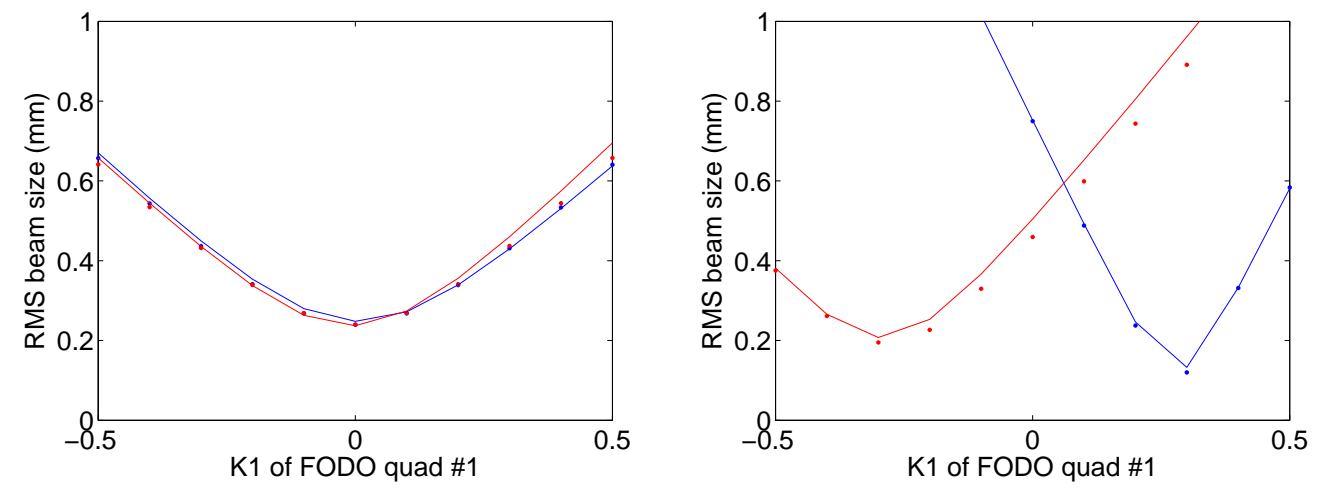

Figure 3.18: RMS beam size evolution as a function of scanned quadrupole strength $k_{1}$ with [left] and without [right] satisfying the condition $\frac{\alpha_{x, y}}{\beta_{x, y}}=\frac{1}{D}$ and for simulated $20 \mu \mathrm{m}$ resolution and $10 \%$ of RMS beam jitter/statistical randomness, for a normalized $x$ emittance $\epsilon_{x}$ of $4.62 \mu \mathrm{m}$ (blue) and a normalized $y$ emittance $\epsilon_{y}$ of $4.67 \mu \mathrm{m}$ (red). On both plots, the solid lines represent least-squares fits.

Finally, the system

$$
\left(\begin{array}{c}
\sigma_{\text {meas }, 1} \\
\vdots \\
\sigma_{\text {meas }, n}
\end{array}\right)=\left(\begin{array}{ccc}
R_{11,1}^{2} & -2 R_{11,1} R_{12,1} & R_{12,1}^{2} \\
\vdots & \\
R_{11, n}^{2} & -2 R_{11, n} R_{12, n} & R_{12, n}^{2}
\end{array}\right)\left(\begin{array}{c}
\beta_{\text {meas }} \epsilon_{\text {meas }} \\
\alpha_{\text {meas }} \epsilon_{\text {meas }} \\
\gamma_{\text {meas }} \epsilon_{\text {meas }}
\end{array}\right)
$$

is solved by a least-squares fit to recover $\alpha_{\text {meas }}, \beta_{\text {meas }}$ and $\epsilon_{\text {meas }}$, where $R_{11}$ are $R_{12}$ are the $x-x$ and $p_{x}-x$ transfer matrix elements, respectively, which are related to the strengths of the quadrupole magnets used in the scans. The initial parameters $\alpha_{0}, \beta_{0}$ and $\epsilon_{0}$ are compared with the inferred beam from the simulation of the measurements $\alpha_{\text {meas }}, \beta_{\text {meas }}$ and $\epsilon_{\text {meas }}$. The figure of merit $\frac{\epsilon_{\text {meas }}}{\epsilon_{0}}-1$ is plotted in 
Figure 3.19. For these simulations, $\sigma_{\text {res }}=20 \mu \mathrm{m}$. Our calculation follows that derived in Reference [29] using the uncertainty equal to the resolution, $\Delta \sigma=20 \mu \mathrm{m}$.

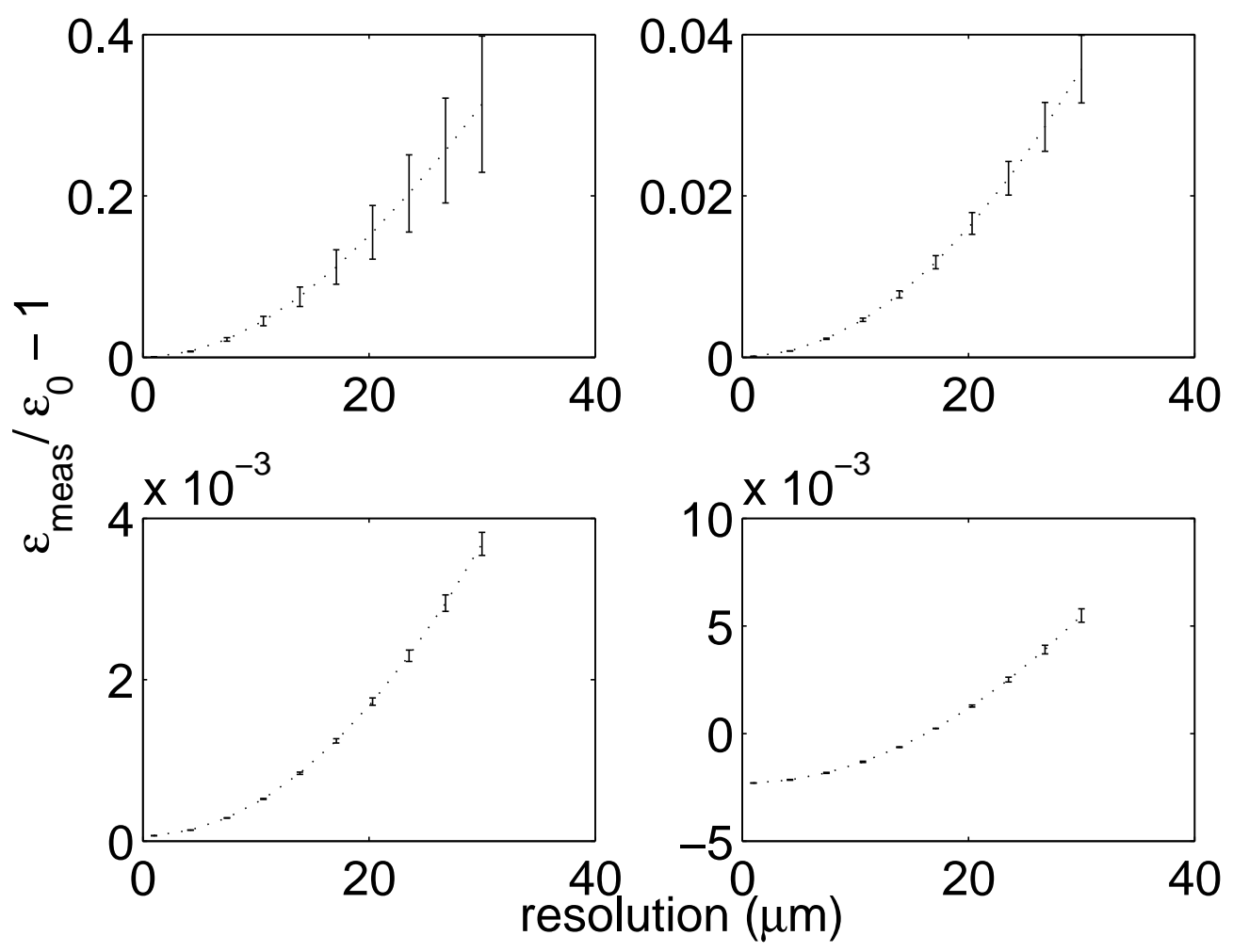

Figure 3.19: Relative error on measured emittance $\epsilon_{\text {meas }}$ with respect to initial emittance $\epsilon_{0}$. The value of $\epsilon_{\text {meas }}$ is obtained from a full simulation of the quadrupole magnet field scan technique described in this note. The four plots correspond to initial emittance values of 0.1 (upper left), 1.0 (upper right), 10.0 (lower left), and 4.6 (lower right) $\mu \mathrm{m}$, with a simulated resolution of $\Delta \sigma=20 \mu \mathrm{m}$, without random jitter.

For low emittance values, the retrieved emittance is overestimated, and the associated error bars are large (on the order of 20\%). A histogram of 5000 simulations with random RMS jitter equal to $10 \%$ of measured beam (following the procedure presented in Reference [32]) gives an indication of what type of statistical variation we can expect in our measurements (Figure 3.20). 

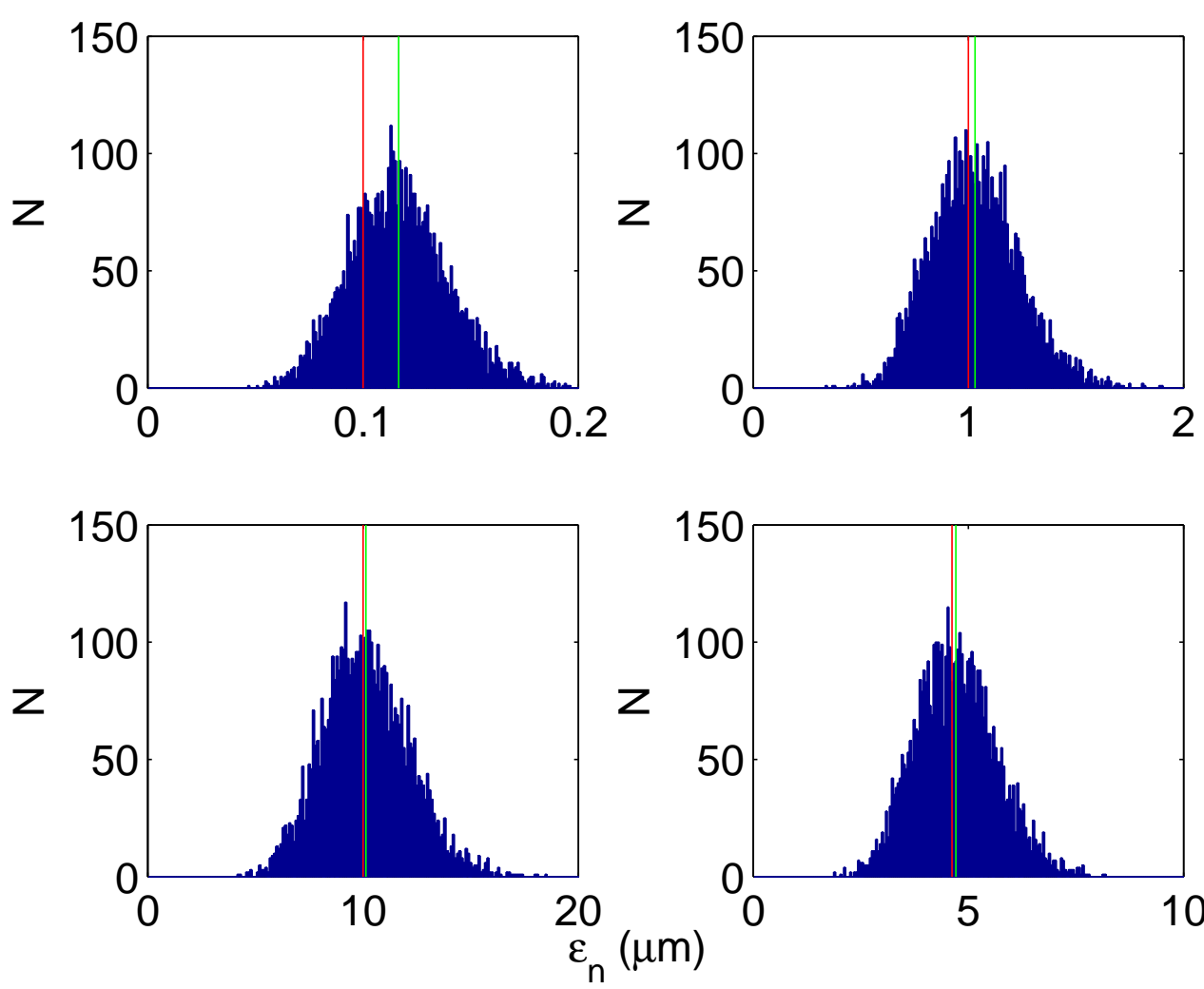

Figure 3.20: Histograms of 5000 emittance measurements for 0.1 (upper left), 1.0 (upper right), 10.0 (lower left), and 4.6 (lower right) $\mu \mathrm{m}$ emittances, with a simulated resolution of $\Delta \sigma=20 \mu \mathrm{m}$, and an uncertainty factor $10 \%$ the RMS beam size, showing the statistical deviation that may be expected in the emittance measurements. The red lines are the simulated normalized emittances, while the green lines are the mean of the 5000 measurements.

\subsection{Quadrupole Magnet Scan Experiment at A0}

In the weeks prior to the shutdown of the A0 photoinjector in 2011, a camera that would eventually go on to be used at ASTA and the developed software were tested using the A0 beamline. The camera was installed at Radiabeam Cross XUR1. The quadrupole that we used to perform the scan was Q3AX14. The bunch charge used during the measurement was 150pC. The experimental setup is shown in Figure 3.21. 

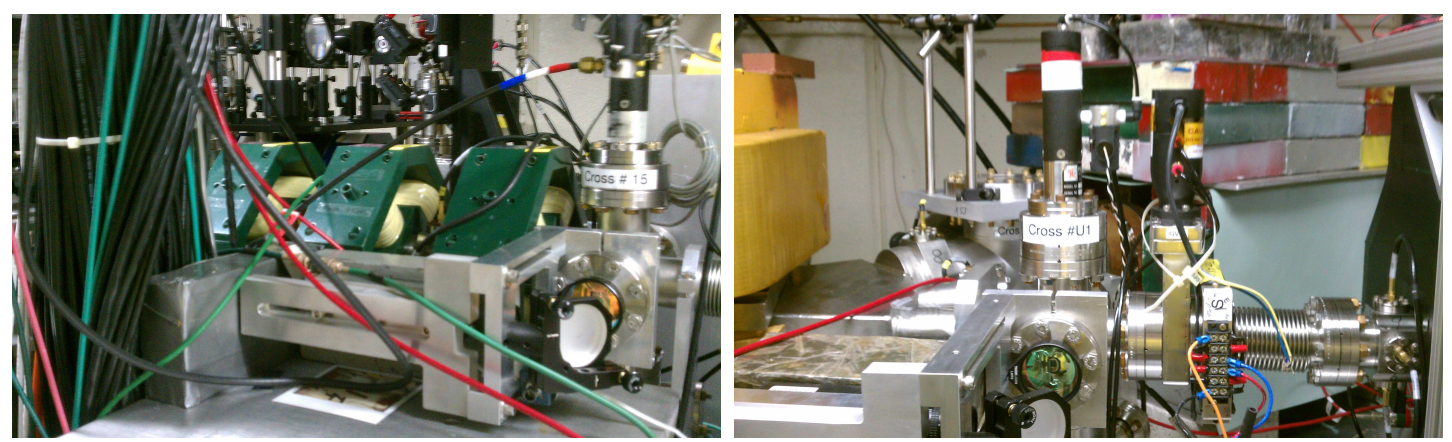

Figure 3.21: Experimental configuration at A0. (left) The quadrupole magnets used for the scan. (Right) The cross containing the screen on which the images were taken.

The developed software was designed to determine the RMS width of the signal in either $\mathrm{X}$ and $\mathrm{Y}$, in units of pixels. Using a test screen (shown in Figure 3.22, I was able to determine the conversion rate of 8.85 microns per pixel, or 113 pixels per millimeter. With the beam on, we then focused the beam in $x$.
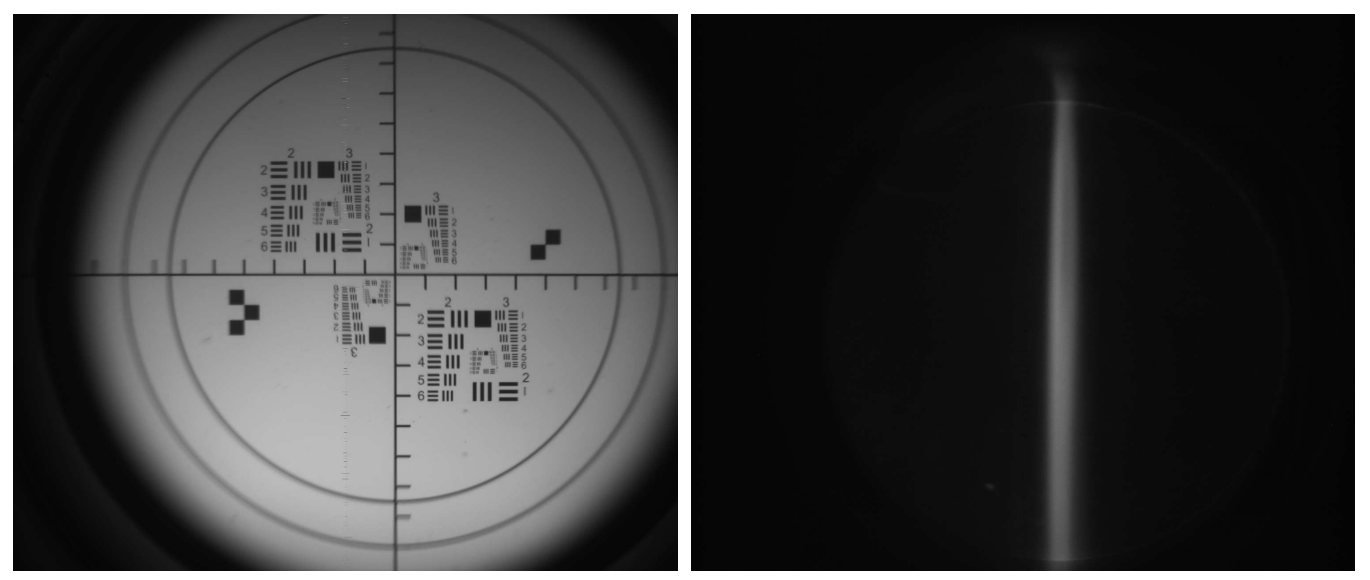

Figure 3.22: Images taken from the screen via the capture software. (left) the calibration screen used to figure out the correspondence between pixels and spatial size, as illuminated by a flashlight. (right) The beam hitting the OTR screen, used for the quadrupole scan measurement, with the scale as indicated on the calibration screen. The measured dimension here is $x$, which grows and shrinks as the quadrupole magnet strengths are changed.

Prior to the scan, I performed an automatic de-gaussing process on the quadrupoles to be used in the measurement. I recorded the current in Amps for each point in the 
scan, and then used the known conversion of $0.79 \mathrm{~T} / \mathrm{A}$ to calculate the K1 value. Once the $\mathrm{K} 1$ value is known, the $R_{11}$ values and $R_{12}$ values of the quadrupole-plusdrift system from the magnet to the screen are calculated and a least squares fit is performed to calculate the emittance $\varepsilon_{x}$, from Eq. 3.13.

$$
\sigma_{x}=\sqrt{\varepsilon_{x}\left(\beta R_{11}^{2}+2 \alpha R_{12} R_{12}+R_{12}^{2} \frac{1+\alpha}{\beta}\right)} .
$$

The least-squares fit was performed using the built-in MatLAB [33] functions, though equivalent functions exist in nearly any other scripting language.

Due to time limitations in the weeks leading to the September 30th, 2011 shutdown of the original A0 beamline, we performed the scan only for the $x$ dimension. While it is possible to scan both dimensions simultaneously, it requires specific quadrupole strength setting at for several magnets, which was not viable for the limited time-frame. In simulations of the similar configuration at ASTA, we retuned the upstream quadrupoles such that both $x$ and $y$ could be scanned at the same time, i.e., their local minimas in plots of beam-size versus the focusing parameter K1 overlapped. The data from the scan is presented in Table 3.2.

I plotted the data in MATLAB, and performed the least-squares fit, which is shown in Figure 3.23.

The emittance measurement agrees reasonably well with the slit measurement camera method. The calculated value of $\varepsilon_{x}$ based on the quadrupole scan was $3.03 \mu \mathrm{m}$. The value given by another system in the beamline, the streak camera, was $3.72 \mu \mathrm{m}$. The discrepancy can be attributed to several issues. These include the limited number of points used for the scan, the asymmetry of the scan about 
Table 3.2: Data from A0 quadrupole scan.

\begin{tabular}{cccccc}
\hline \hline Quad. Curr. (A) & $\mathrm{K} 1$ & $R_{11}$ & $R_{12}$ & $\sigma_{x}$ (Pixels) & $\sigma_{x}(\mathrm{~mm})$ \\
\hline .299 & 4.66 & 2.01 & -2.04 & 113 & 1.0 \\
.340 & 5.30 & 2.15 & -2.03 & 84 & 0.7 \\
.380 & 5.92 & 2.28 & -2.03 & 57 & 0.5 \\
.420 & 6.55 & 2.42 & -2.02 & 25.5 & 0.2 \\
.460 & 7.17 & 2.55 & -2.01 & 21.5 & 0.2 \\
.500 & 7.79 & 2.68 & -2.01 & 40 & 0.4 \\
.540 & 8.42 & 2.81 & -2.00 & 67 & 0.6 \\
.580 & 9.04 & 2.95 & -1.99 & 98 & 0.9 \\
.620 & 9.67 & 3.08 & -1.99 & 133 & 1.2 \\
.660 & 10.29 & 3.21 & -1.98 & 162 & 1.4 \\
.700 & 10.91 & 3.34 & -1.97 & 220 & 1.9 \\
\hline \hline
\end{tabular}

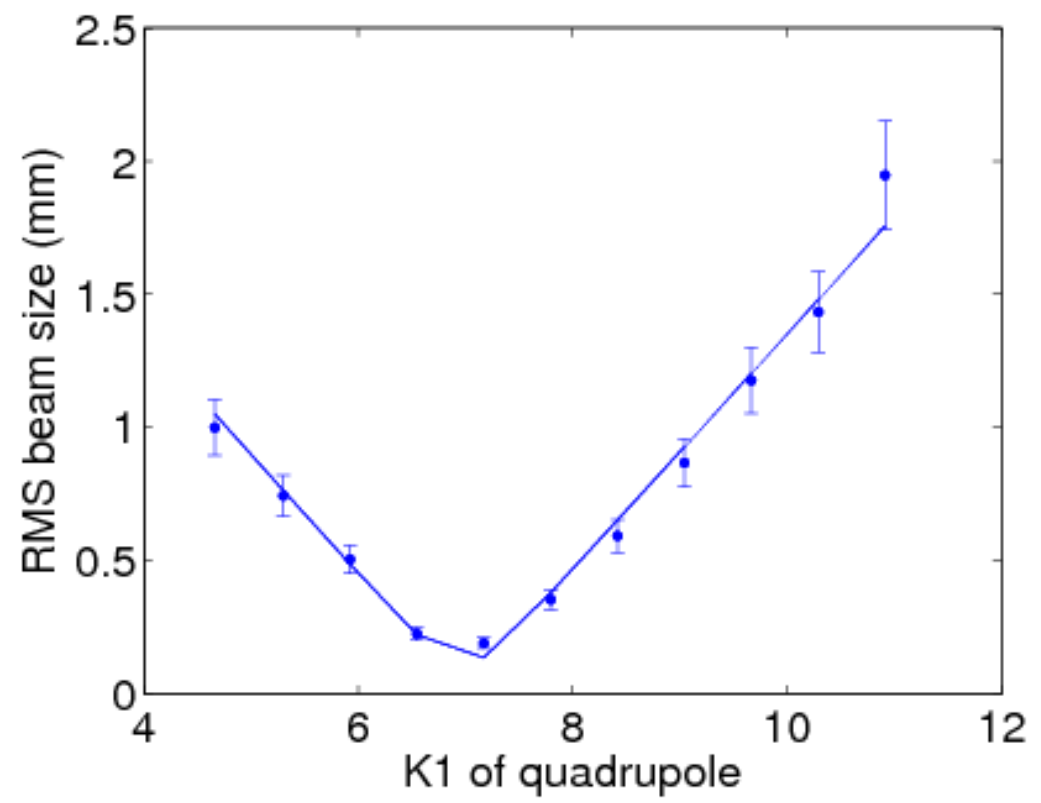

Figure 3.23: Data from the A0 quadrupole magnet scan experiment, RMS beam size vs. K1 setting of the quadrupole magnet in units of $\frac{1}{m^{2}}$. The line is a least-square fit that was performed in MATLAB. 
the minima, and that I did not take the average of many data samples to account for beam jitter. The data shown in the figure is not in perfect agreement, and is worse than the simulated data-set that was used for Figure 3.18. 


\section{CHAPTER 4}

\section{NUMERICAL STUDIES OF LOW-ENERGY BUNCH COMPRESSION AT ASTA}

The compression of low-energy bunches prior to the entrance of the cryomodules is necessary for the creation of short, high-brightness bunches. As discussed in

Chapter 3, the ASTA facility contains a bunch compressor at low-energies along with room for an additional one at high-energies, downstream of the cryomodules. To investigate the range of beam parameters that could be used for user experiments at both high- and low- energies, I performed a detailed study of the low-energy bunch compressor under the influence of collective effects.

\subsection{Introduction to Bunch Compression and Bunch Compressor Design}

The compression of $\mathrm{a} \sim 40-\mathrm{MeV}$ electron bunch via magnetic compression is investigated for the case of the ASTA photoinjector diagrammed in Figure 4.1, though the final beam energy has been upgraded to $\sim 50-\mathrm{MeV}$ after this study was completed in the Fall of 2012. The parameters used for the study are based on this in the simulation overview discussed in Chapter 3.

The ASTA bunch compressor consists of four 0.2-m rectangular dipoles (B1, B2, B3, B4) with respective bending angles of $(+,-,-,+) 18^{\circ}$. The longitudinal dispersion of $\mathrm{BC} 1$ is $R_{56}=-0.19 \mathrm{~m}$. Downstream of the bunch compressor is a a single-shot 
LPS diagnostic system [34]. that combines a transverse-deflecting cavity (TDC) with a vertical spectrometer, and is discussed further in Chapter 5.

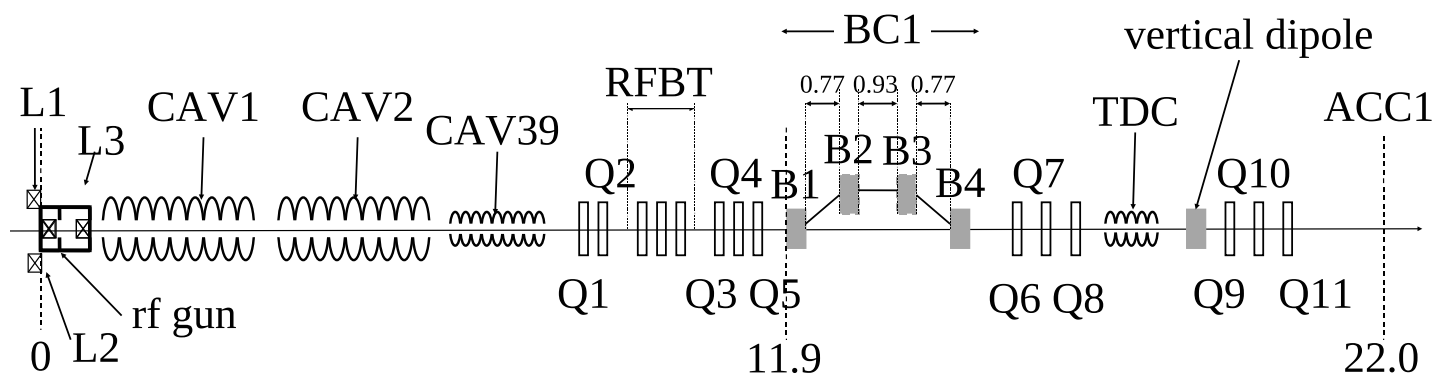

Figure 4.1: Injector configuration at ASTA. The "RF gun", "L1" and "L2" respectively correspond to the gun cavity and surrounding solenoid magnets, "CAV1", "CAV2", and "CAV39" are superconducting RF cavities, "RFBT" is the round-toflat beam transformer, and "BC1" refers to the magnetic bunch compressor, and B1-4 are the dipoles of the chicane, with distance between the dipoles marked in the figure. The number below the beamline indicates the axial positions in meters w.r.t. the photocathode surface.

The beam dynamics through CAV2 were simulated with ASTRA and optimized using a genetic optimizer for several cases of charge and photocathode drive-laser configurations [?]. The resulting phase space distributions are used as a starting point for transport and compression through the beamline downstream of CAV39. The quadrupoles settings were optimized for the various operating charges using the single-particle dynamics program ELEGANT [35].

In this chapter, I explore the performance of the $\mathrm{BC} 1$ low-energy bunch compressor for various bunch charges. I focus on investigating a possible trade-off between high-peak current and transverse-emittance preservation, a problem is vital to many electron accelerators. The numerical studies are performed with several models to account for CSR and/or SC available in the beam dynamics program ELEGANT, IMPACT-Z, and CSRTRACK; overviews of the simulation codes are presented in Ap- 
pendix C. The programs used for our simulations are, when possible, benchmarked against each other.

\subsection{Longitudinal Beam Dynamics in the Injector}

Prior to injection in the cryomodule, the bunch can be longitudinally compressed using the magnetic bunch compressor (BC1) which, consists of four 0.2-m rectangular dipoles (B1, B2, B3, B4) with bending angles of $(+,-,-,+) 18^{\circ}$; see Figure 4.1 for distances between the dipoles. This study is based on an early design for the low-energy bunch compressor; the chicane's dipole lengths and drift distances may change for the final experiment, and the bunch energy in the injector may be increased up to $\sim 50 \mathrm{MeV}$. In this process, an electron has its initial LPS coordinate $\left(z_{0}, \delta_{0}\right)$ upstream of $\mathrm{BC} 1$ mapped to the final LPS coordinates downstream of BC1

$$
\left\{\begin{array}{l}
z_{f}=z_{0}+R_{56} \delta_{0} \\
\delta_{f}=\delta_{0}
\end{array}\right.
$$

where $R_{56}$ is the longitudinal dispersion associated with $\mathrm{BC} 1$ taken to be negative for a chicane-type compressor. Here we neglect possible collective effects and only considered the first-order transfer map. The final root-mean-square (RMS) bunch length is

$$
\begin{aligned}
\sigma_{z, f} & =\left[\sigma_{z, 0}^{2}+R_{56}\left\langle z_{0} \delta_{0}\right\rangle+R_{56}^{2} \sigma_{\delta, 0 u}^{2}\right]^{1 / 2} \\
& =\sigma_{z, 0}\left[1+R_{56} \mathcal{C}+R_{56}^{2} \frac{\sigma_{\delta, 0 u}^{2}}{\sigma_{z, 0}^{2}}\right]^{1 / 2}
\end{aligned}
$$


Therefore, with appropriate initial LPS $\operatorname{chirp}, \mathcal{C} \equiv-\left\langle z_{0} \delta_{0}\right\rangle / \sigma_{z, 0}^{2}$, the bunch compression can be varied. When $\mathcal{C}=-1 / R_{56}$ the bunch length reach its minimal value limited by the uncorrelated fractional momentum spread $\sigma_{\delta, 0 u}$. The $R_{56}$ of the chicane is $19 \mathrm{~cm}$, which indicates that the greatest compression for a perfectly linearized beam will occur for $\mathcal{C} \approx 5.25 \mathrm{~m}^{-1}$. We operate CAV1 on-crest, and use only-CAV2 to impart the energy-chirp, to avoid velocity bunching and its complications. Therefore a simple model of the longitudinal dynamics can be developed.

Consider an electron emitted by the RF gun with energy and position related by

$$
E(z)=E_{g}+a z+b z^{2},
$$

where $E_{g}, a$, and $b$ are parameters that depend on the RF gun operating parameters. Taking the average beam energy to be $E_{g}$ downstream of the gun and considering the acceleration voltage through CAV1 and CAV2 to be

$$
V(z)=V_{1} \cos \left(k z+\varphi_{1}\right)+V_{2} \cos \left(k z+\varphi_{2}\right),
$$

and expanding in $z$, we have (to second order) the energy gain from CAV1 and CAV2

$$
\begin{aligned}
\Delta E_{1+2}(z) & =e V_{1} \cos \left(\varphi_{1}\right)+e V_{2} \cos \left(\varphi_{2}\right) \\
& -e V_{1} k z \sin \left(\varphi_{1}\right)-e V_{2} k z \sin \left(\varphi_{2}\right) \\
& -\frac{1}{2} e V_{1} k^{2} z^{2} \cos \left(\varphi_{1}\right)-\frac{1}{2} e V_{2} k^{2} z^{2} \cos \left(\varphi_{2}\right) .
\end{aligned}
$$


The total energy downstream of CAV2 is then

$$
\begin{aligned}
E(z) & =E_{g}+a z+b z^{2}+V_{1} \cos \left(\varphi_{1}\right)+V_{2} \cos \left(\varphi_{2}\right) \\
& -V_{1} k z \sin \left(\varphi_{1}\right)-V_{2} k z \sin \left(\varphi_{2}\right) \\
& -\frac{1}{2} e V_{1} k^{2} z^{2} \cos \left(\varphi_{1}\right)-\frac{1}{2} e V_{2} k^{2} z^{2} \cos \left(\varphi_{2}\right) .
\end{aligned}
$$

Linearization of the LPS requires that the sum of the $z^{2}$ terms goes to zero, which we accomplish with a third harmonic cavity, CAV39, operating at $3.9 \mathrm{GHz}$, which can be similarly expanded as

$$
\Delta E_{39}(z)=e V_{39} \cos \left(\varphi_{39}\right)-e V_{39} k z \sin \left(\varphi_{39}\right)-\frac{1}{2} e V_{39} k_{39}^{2} z^{2} \cos \left(\varphi_{39}\right)
$$

If we are to use CAV39 for linearization, we then have the requirement

$$
b-\frac{1}{2} e V_{1} k^{2} \cos \left(\varphi_{1}\right)-\frac{1}{2} e V_{2} k^{2} \cos \left(\varphi_{2}\right)-\frac{1}{2} e V_{39} k_{39}^{2} \cos \left(\varphi_{39}\right)=0
$$

which can be rearranged

$$
V_{39} \cos \left(\varphi_{39}\right)=\frac{1}{9}\left[\frac{2 b}{e k^{2}}-V_{1} \cos \left(\varphi_{1}\right)-V_{2} \cos \left(\varphi_{2}\right)\right]
$$

In addition to the linearization, we have a second condition imposed by our requirement of maximum compression, so that the terms linear in $z$, i.e. the LPS chirp, is related to the $R_{56}$ of the chicane:

$$
\frac{a-e V_{1} k \sin \left(\varphi_{1}\right)-e V_{2} k \sin \left(\varphi_{1}\right)-e V_{39} 3 k \sin \left(\varphi_{39}\right)}{E_{g}+e V_{1} \cos \left(\varphi_{1}\right)+e V_{2} \cos \left(\varphi_{2}\right)+e V_{39} \cos \left(\varphi_{39}\right)}=-\frac{1}{R_{56}}
$$


Substituting Equation 4.8 into the denominator gives

$$
\frac{a-e V_{1} k \sin \left(\varphi_{1}\right)-e V_{2} k \sin \left(\varphi_{2}\right)-e V_{39} 3 k \sin \left(\varphi_{39}\right)}{E_{g}+\frac{8}{9} e V_{1} \cos \left(\varphi_{1}\right)+\frac{8}{9} e V_{2} \cos \left(\varphi_{2}\right)+\frac{2 b}{9 k^{2}}}=-\frac{1}{R_{56}}
$$

Rearranging to isolate $V_{39} \sin \left(\varphi_{39}\right)$, we find

$$
\begin{aligned}
V_{39} \sin \left(\varphi_{39}\right)= & \frac{1}{3 k R_{56}}\left[E_{g}+\frac{8}{9} e V_{1} \cos \left(\varphi_{1}\right)+\frac{8}{9} e V_{2} \cos \left(\varphi_{2}\right)+\frac{2 b}{9 k^{2}}\right] \\
& +\frac{1}{3 k} V_{1} \cos \left(\varphi_{1}\right)+\frac{1}{3 k} V_{2} \cos \left(\varphi_{2}\right)-\frac{1}{3 k} a .
\end{aligned}
$$

Combining Eqs. 4.8 and 4.12, we obtain the expression for the operating phase,

$$
\varphi_{39}=\tan ^{-1}\left(\frac{V_{T}}{V_{B}}\right) .
$$

where

$$
\begin{aligned}
V_{T} & =\frac{1}{3 k R_{56}}\left[E_{g}+\frac{8}{9} e V_{1} \cos \left(\varphi_{1}\right)+\frac{8}{9} e V_{2} \cos \left(\varphi_{2}\right)+\frac{2 b}{9 k^{2}}\right]+\frac{1}{3 k} V_{1} \cos \left(\varphi_{1}\right)+\frac{1}{3 k} V_{2} \cos \left(\varphi_{2}\right)-\frac{1}{3 k} a, \\
V_{B} & =\frac{1}{9}\left[\frac{2 b}{e k^{2}}-V_{1} \cos \left(\varphi_{1}\right)-V_{2} \cos \left(\varphi_{2}\right)\right] .
\end{aligned}
$$

The recovered operating phase can then be substituted back into Equation 4.8 to find the voltage.

We now consider our nominal operating condition where CAV1 is on-crest $\left(\varphi_{1}=\right.$ 0 ) and CAV2's phase $\varphi_{2}$ is used to impart the LPS chirp. Typical operating parameters for the ASTA photoinjector are $E_{g}=5 \mathrm{MeV}, V_{1}=12 \mathrm{MeV}$, and $V_{2}=22 \mathrm{MeV}$, and we take $\varphi_{2}=-27^{\circ}$. For simplicity, we ignore initial LPS linear and quadratic terms arising from the $\mathrm{rf}$ gun $(a=b=0)$. Following the previous equations, the phase and voltage for CAV39 are then $\varphi_{39}=170^{\circ}$ and $V_{39}=3.6 \mathrm{MeV}$. 
Since we will not always be phasing the cavities to achieve maximum compression, we show in Figure 4.2 the operating phase $\phi_{39}$ and voltage $V_{39}$ for CAV39 to linearize the LPS as functions of the phase of CAV2.
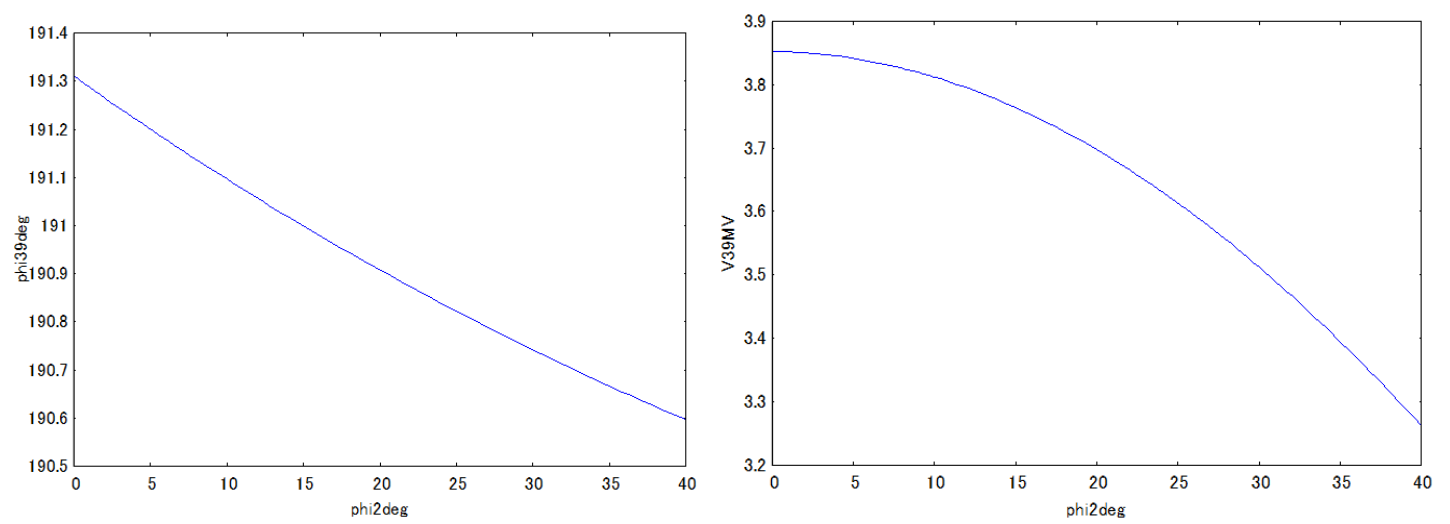

Figure 4.2: The operating phase $\phi_{39}$ (left) and voltage $V_{39}$ (right) for CAV39 as functions of the operating phase of CAV2, $\phi_{2}$, using its voltage $V_{2}=22 \mathrm{MeV}$.

When collective effects are taken into account the beam dynamics can significantly deviate from the simple model just discussed. In the case of $\mathrm{BC} 1$ in particular, because the electrons travel on a curved trajectory while within the field of a dipole magnet, they emit synchrotron radiation. This radiation leads to a net energy loss (which is insignificant in the case of $\mathrm{BC} 1$ ) but can also feedback on the beam and introduce an energy redistribution.

\subsection{Bunch Compressor Beam Optics}

The code ELEGANT was used to model and optimize the single-particle dynamics throughout the ASTA beamline. Two examples of LPS distributions tracked through $\mathrm{BC} 1$ for an ideal compression, i.e. in absence of collective effects, appear in Figure 4.3. The simulations, carried out with ELEGANT, illustrate the benefits of 
the LPS linearization using CAV39 toward significantly improving the peak current. For these simulations, the LPS is modeled upstream of CAV2 with ASTRA as a 3.2$\mathrm{nC}$ bunch, which is then loaded into ELEGANT with matching functions performed via GlueTrack, with and without the linearization process detailed earlier. The linearization of the LPS produced by a 3.9-GHz cavity is modeled with the transformation $\delta \rightarrow \delta_{0}-b z_{0}^{2}$ via a set of Python scripts called GlueTrack, where the parameter $b$ is obtained from a polynomial fit of the incoming LPS distribution $\left(z_{0}, \delta_{0}\right)$. GlueTrack is also used to match the Courant-Snyder (C-S) parameters and energies of the distributions, and to adjust the energy chirp of the bunch, as the original AstRA simulations which were used to generate the different distributions were not optimized to produce distributions of equal energy or C-S parameters, and because re-running the ASTRA simulations to generate new distributions would be time-prohibitive.

Without collective effects, the peak currents for a linearized $3.2 \mathrm{nC}$ bunch may exceed $12 \mathrm{kA}$, and is limited by the uncorrelated energy spread $\hat{I} \simeq \frac{Q c}{R_{56} \sigma_{\delta u} \sqrt{2 \pi}}$, where $\sigma_{\delta u}$ is the uncorrelated fractional momentum spread.

To determine the optimal C-S parameters for the minimization of CSR effects, we performed a scan of initial C-S parameters using CSRTRACK's 1DP model (shown in Figure 4.4) to find the operating regimes of least emittance-growth. The "valley" of minimum growth in the lower-right hand corner of the plot coincides with the C-S parameters that lead to a waist between the third and fourth dipole, at which its "projected" slice length is greatest [36].

The upper "valley" of the scan corresponds to a waist between the 1st and 2nd dipoles, though simulations show that such a solution may lead to large-valued betatron functions at the chicane exit which would complicate the beam optics downstream of the chicane and render the lattice more prone to aberrations, including 

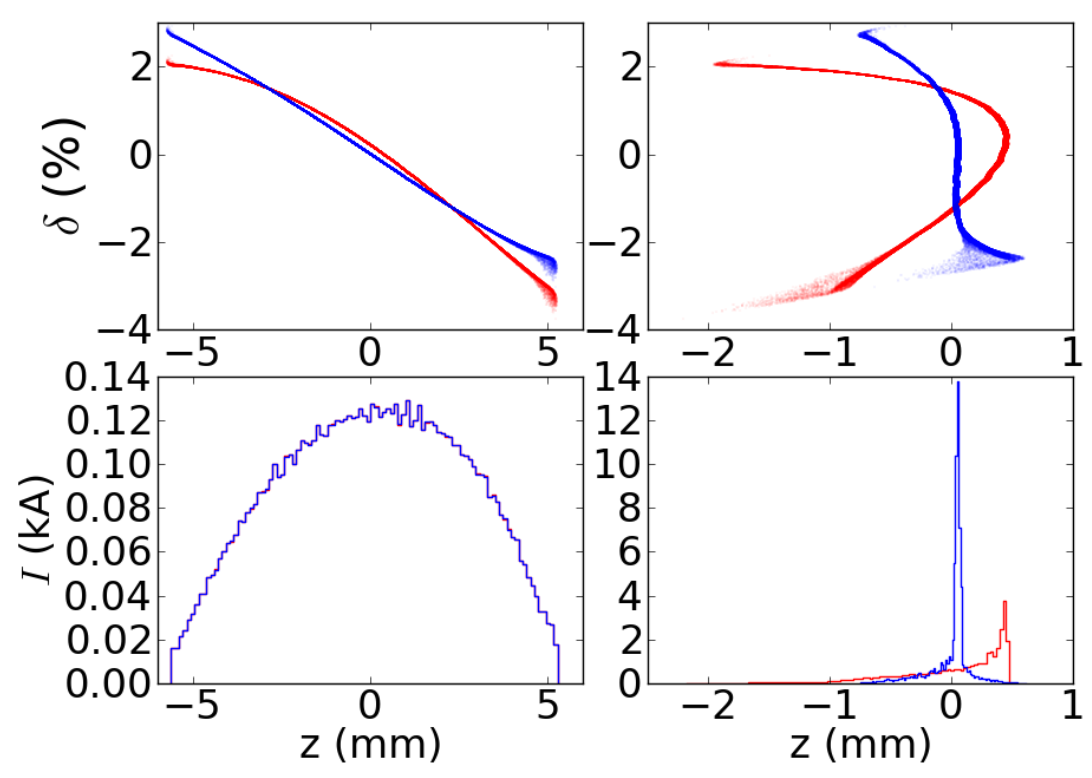

Figure 4.3: LPS distributions (top row) and associated current profiles (bottom row) before (left column) and after (right column) BC1, without collective effects. The red and blue traces correspond respectively to the linearized and nominal initial LPS of a 3.2-nC bunch, showing the vastly higher peak currents that become available by using the third-harmonic cavity for LPS linearization.

second-order effects, which are included for the simulations presented here. This is seemingly a byproduct of the specific LPS structure of the distribution used, and disappears for scans with completely-linearized bunches, such as those generated directly from ELEGANT

The beam dynamics through CAV2 were simulated with ASTRA and optimized using a genetic optimizer for several cases of charge and photocathode drive-laser configurations; see Reference [?]. The resulting phase space distributions are used as a starting point for transport and compression through the beamline downstream of CAV39. The quadrupoles settings were optimized for the various operating charges using the single-particle dynamics program ELEGANT. The evolution of the nominal 

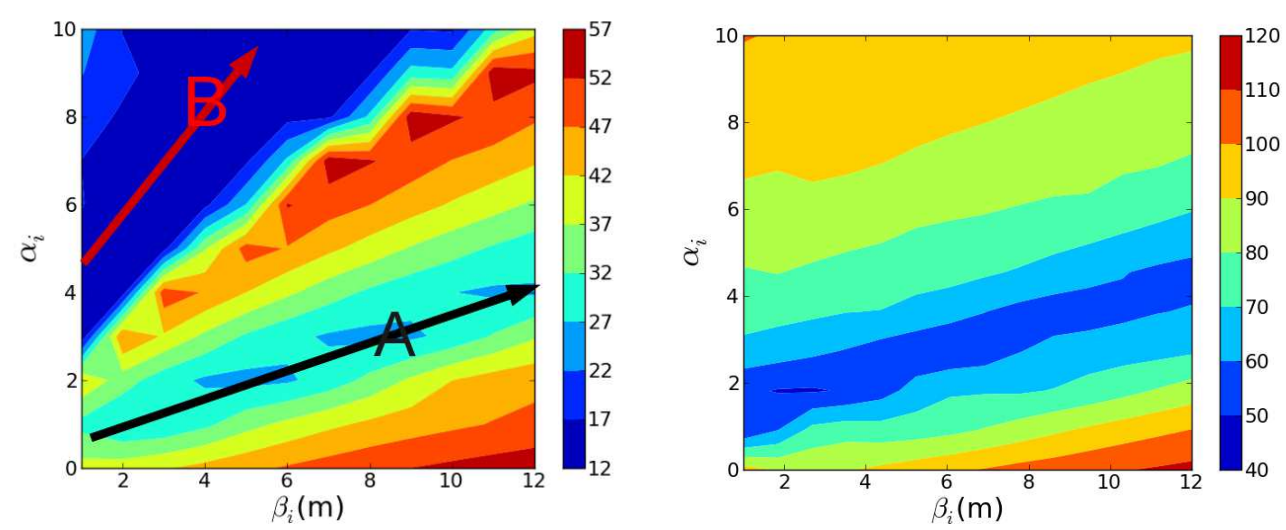

Figure 4.4: Contour plot of the final normalized horizontal emittance $\left(\varepsilon_{x}\right.$ in $\left.\mu \mathrm{m}\right)$ a function of the initial C-S parameters $\beta_{i}$ and $\alpha_{i}$ for the nominal (left) and linearized (right) incoming LPS. The simulations are performed with CSRTRACK's 1D-Projected model. The black arrow indicates a region where the beam reaches a waist in $\mathrm{x}$ between the 3rd and 4th dipoles, and the red arrow indicates where the beam reaches a waist between the 1st and 2 nd dipoles. The label "A" indicates the operating point for most of the simulations performed in this report.

betatron functions downstream of CAV39 up to the cryomodule entrance is plotted in Figure 4.5.

Simulations were performed for four cases of bunch charges ranging from $3.2 \mathrm{nC}$ to $20 \mathrm{pC}$. For each charge the transverse emittance was optimized with ASTRA [?]. The distributions were manipulated using GLUETRACK to adjust several parameters including their Courant-Snyder (C-S) parameters, and longitudinal phase space (LPS) $\operatorname{chirp} \mathcal{C} \equiv-\left\langle z_{i} \delta_{i}\right\rangle / \sigma_{z, i}^{2}$ where $\left(z_{i}, \delta_{i}\right)$ are the coordinates in the LPS, the $\langle u\rangle$ indicates the statistical averaging of variable $u$ over the LPS distribution. We also modeled the effect of CAV39 by numerically removing the second-order correlation in the LPS distribution. While a realistic model of the cavity should include ponderomotive RF focusing [?], this contribution is relatively small in our case, and does not result in appreciable emittance growth. The impact of transverse focusing would be only a slight changes in the initial C-S parameters that would need to 


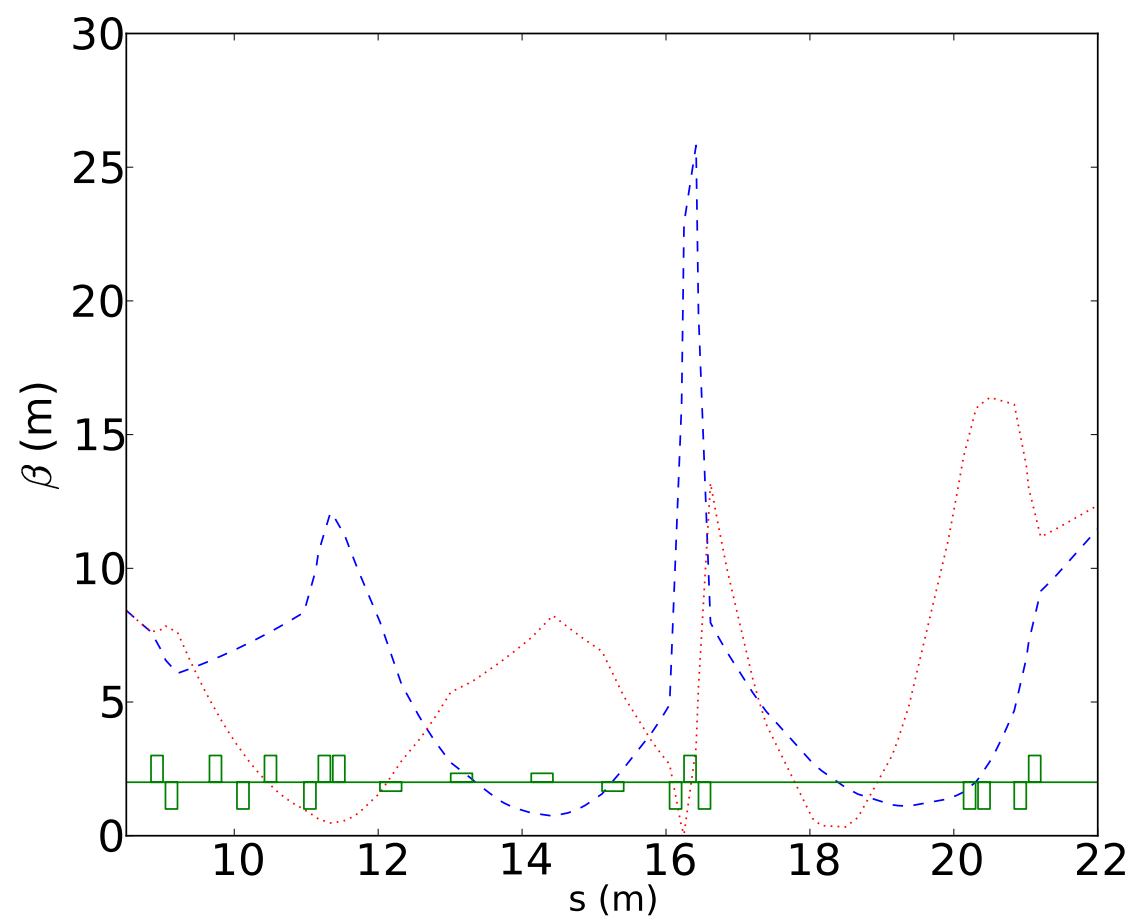

Figure 4.5: Evolution of the horizontal (dashed line) and vertical (dotted line) betatron functions through the ASTA injector. The lower line and rectangles indicate the location of quadrupole and dipole (smaller rectangles) magnets. The BC1 compressor is located at $s \in[11.9,15.1] \mathrm{m}$. The origin of the horizontal axis $(s=0 \mathrm{~m}$, not shown) corresponds to the photocathode surface.

be matched to the nominal parameters of the bunch compressor using the first few quadrupole magnets.

The charge-independent beam parameters computed 0.1-m upstream of dipole magnet B1 are summarized in Table 4.1. The beam distribution was matched to achieve the transverse C-S parameters listed in Table 4.1 (see discussion below) upstream of BC1. The initial LPS chirp was tuned by removing the second-order correlation (as discussed earlier) and adding a first-order correlation between the LPS coordinates such that the final fractional momentum offset of a particle depends on its position following $\delta_{i, c}=\delta_{i, u}+\mathcal{C} z_{i}$ where $\delta_{i, u}$ is the uncorrelated fractional mo- 
Table 4.1: Transverse and longitudinal beam parameters 0.1-m upstream of B1 dipole entrance face. Only the Courant-Snyder parameters were fixed while the other parameters depend on the bunch charge or upstream beamline settings.

\begin{tabular}{ccc}
\hline Parameter & Value & Units \\
\hline \hline$\beta_{x, i}$ & 8 & $\mathrm{~m}$ \\
$\alpha_{x, i}$ & 3 & - \\
$\beta_{y, i}$ & 1.6 & $\mathrm{~m}$ \\
$\alpha_{y, i}$ & -1.6 & - \\
$\mathcal{C}$ & {$[1.0,6.0]$} & $\mathrm{m}^{-1}$ \\
total energy & 38.6 & $\mathrm{MeV}$ \\
\hline
\end{tabular}

mentum offset obtained after removal of the first and second-order correlation. The other LPS parameters and emittances (shown for the four charges in Table 4.2) are inherent to the generation process and were not adjusted. The initial LPS distribution for each of the four charges appears in Figure 4.6 with its linear correlation removed. The $S$-shaped LPS is a remnant of space charge effects during the bunch generation and transport before acceleration in CAV1 and CAV2 [37]. As expected, larger charges yield higher total fractional momentum spread.

Table 4.2: Initial normalized transverse $\varepsilon_{x / y, i}$ and longitudinal $\varepsilon_{z, i}$ emittances and RMS bunch length $\sigma_{z, i}$ for the four cases of charge considered in this paper. The parameters are computed 0.1-m upstream of dipole magnet B1's entrance face.

\begin{tabular}{ccccc}
\hline $\mathrm{Q}(\mathrm{nC})$ & $\varepsilon_{x, i}(\mu \mathrm{m})$ & $\varepsilon_{y, i}(\mu \mathrm{m})$ & $\varepsilon_{z, i}(\mu \mathrm{m})$ & $\sigma_{z, i}(\mathrm{~mm})$ \\
\hline \hline 3.2 & 4.43 & 4.58 & 82.19 & 2.56 \\
1.0 & 2.20 & 2.22 & 33.41 & 1.95 \\
0.250 & 0.580 & 0.576 & 14.37 & 1.93 \\
0.020 & 0.296 & 0.297 & 2.54 & 1.26 \\
\hline
\end{tabular}

For 3D simulations, such a comprehensive scan was too demanding, so we performed scattered simulations, with several different numbers and sizes for the Gaussian sub-bunches. Table 4.3 shows the results for the simulation models considered 

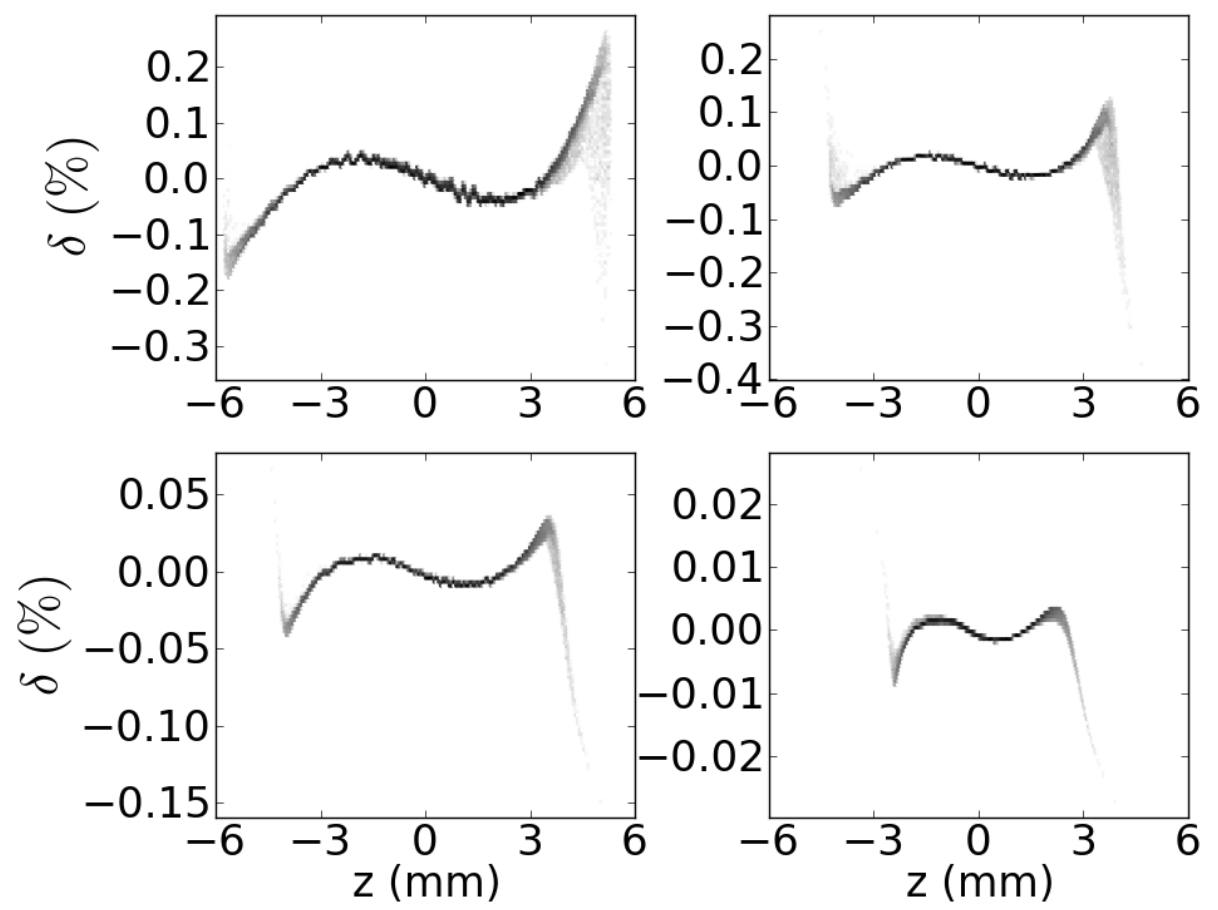

Figure 4.6: LPS distributions 0.1-m upstream of dipole magnet B1's entrance face for 3.2 (a), 1.0 (b), 0.25 (c) and $0.02 \mathrm{nC}$ (d) bunches. The distributions were obtained from simulations of the photoinjector beam dynamics in ASTRA.

in this report. The settings and parameters for a 3.2-nC bunch with linearized LPS are shown. In IMPACT-Z, the number of bins in each of the three dimensions that is used for SC and CSR calculations needed to be determined. Likewise, CSRTRACK requires RMS sizes for the sub-bunches in each of the three dimensions; $\sigma_{h}$, $\sigma_{v}$ and $\sigma_{\|}$are respectively the horizontal, vertical and longitudinal RMS sizes of the sub-bunches. For a more detailed overview of the simulation parameters and justifications for the selected parameters, see the study in Appendix C.

To inspect the numerical variation from the the process of randomly cutting up to $97.5 \%$ of the particles from the initial $200 \mathrm{k}$ distribution, we repeated the 
Table 4.3: Simulated beam parameters downstream of BC1 with IMPACT-Z ("IMPZ") and CSRTRACK ("CSRT") the model used is appended to the program's name. The parameter column and indicates the number of bins, or the absolute or relative (in \%) Gaussian particle size in the longitudinal dimension, $\sigma_{\|}$. These entries were generated for $\mathcal{C}=$ at $5.22 \mathrm{~m}^{-1}$, so that the bunch is near maximum compression.

\begin{tabular}{lccccc}
\hline Model & $N$ & Parameter & $\varepsilon_{x}(\mu \mathrm{m})$ & $\delta(\%)$ & $\hat{I}(\mathrm{~A})$ \\
\hline \hline IMPZ-SC+CSR & $2 \cdot 10^{5}$ & $256^{a}$ & 77.5 & 3.00 & 7.99 \\
\hline CSRT-1DP & $2 \cdot 10^{5}$ & $1 \mu \mathrm{m}$ & 55.4 & 1.85 & 6.16 \\
CSRT-1DP & $2 \cdot 10^{5}$ & $10 \%$ & 54.9 & 1.83 & 8.04 \\
CSRT-1DP & $2 \cdot 10^{5}$ & $5 \%$ & 54.5 & 1.87 & 8.78 \\
CSRT-1DP & $2 \cdot 10^{5}$ & $1 \%$ & 55.3 & 1.87 & 7.73 \\
\hline CSRT-P2P & $5 \cdot 10^{3}$ & $10 \%$ & 101 & 2.81 & 6.37 \\
CSRT-P2P ${ }^{b}$ & $5 \cdot 10^{3}$ & $10 \%$ & 103 & 3.03 & 6.65 \\
CSRT-P2P & $1 \cdot 10^{4}$ & $10 \%$ & 102 & 2.89 & 6.57 \\
CSRT-P2P & $2 \cdot 10^{4}$ & $10 \%$ & 94.6 & 2.91 & 6.44 \\
CSRT-P2P & $3 \cdot 10^{4}$ & $10 \%$ & 98.4 & 2.86 & 6.44 \\
CSRT-P2P & $2 \cdot 10^{4}$ & $5 \%$ & 97.8 & 2.80 & 5.95 \\
\hline
\end{tabular}

${ }^{a}$ number of longitudinal bins; ${ }^{b}$ a different statistical sample of the $5 \times 10^{3}$ particles was used compared to previous line.

simulations several times with different sets of random particles. This effect, over the limited studies performed here, appears to be minimal.

The P2P model in CSRTRACK, with 10\% RMS Gaussian sub-bunches, has little dependence on the number of macroparticles used over the range presented here, owing to the smoothing that the gaussian sub-bunches offer; see Figure 4.7. Simulations with $5 \mathrm{k}$ particles take around an hour to perform, simulations with 10k particles take several hours (feasible for parametric scans), while the 30k-particle simulations take several days on a 48-CPU cluster, which is reasonable for limited studies.

We choose $10 \mathrm{k}$ macroparticles with $\sigma_{B}=0.1 \sigma_{z}$ as the standard for our 3D simulations based on these results. Using a 48-CPUs on the NICADD cluster, these simulations take around three to four hours to complete. Due to the computa- 

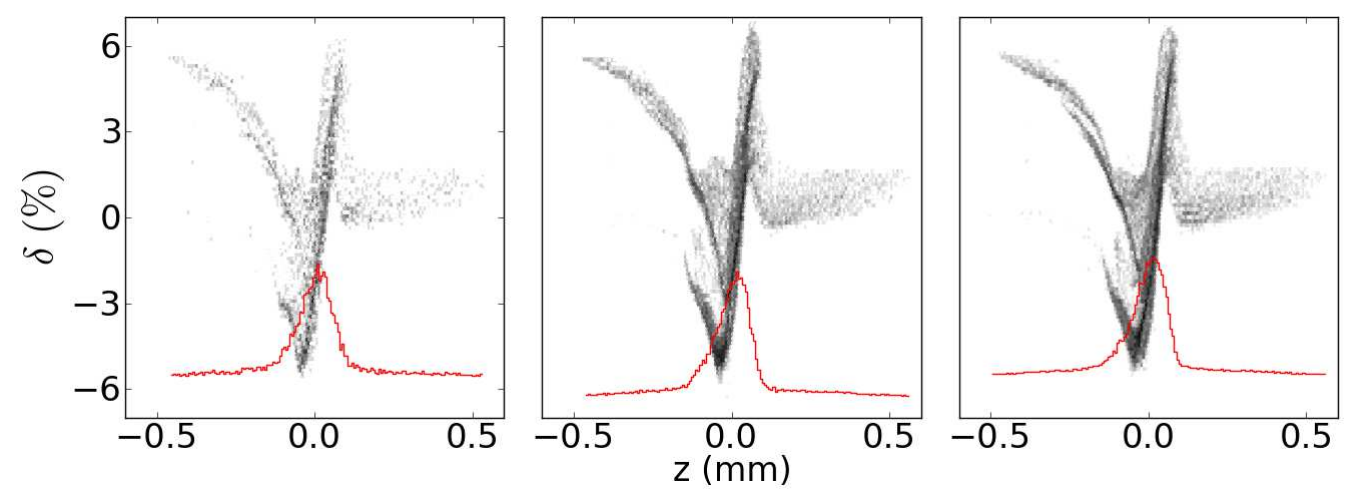

Figure 4.7: LPS downstream of the bunch compressor and associated longitudinal charge distribution (red trace) in arbitrary units using CSRTRACK's P2P model, with $5 \mathrm{k}$ (left), 20k (middle), and 30k (right) macroparticles randomly down-sampled from the same $200 \mathrm{k}$ particle distribution, with $\sigma_{B}=0.1 \sigma_{z}$, for initial LPS chirp of $5.22 \mathrm{~m}^{-1}$ using a $3.2 \mathrm{nC}$ bunch charge. The red line is the longitudinal current projection.

tional intensiveness of the P2P model, only $10^{4}$ sub-bunches were used compared to $2 \times 10^{5}$ used with the 1 DP model. This relatively low number of sub-bunches requires a large $\sigma_{\|}$of $5 \%$ of the longitudinal bunch length $\sigma_{z}$ for the 1DP simulations and $10 \%$ for the P2P simulations. The P2P model requires parameters for the subbunches in the horizontal and vertical dimensions, $\sigma_{h}$ and $\sigma_{v}$. We chose $\sigma_{v}=0.10 \sigma_{y}$ and $\sigma_{\|}=0.10 \sigma_{z}$. However, CSRTRACK does not allow $\sigma_{h}$ to be set as a variable of the horizontal RMS width, so we instead chose $0.1 \mathrm{~mm}$, which is on the order of $10 \%$ of the RMS size $\sigma_{x}$ for all four of the bunch charges presented here. Both of CSRTRACK's models neglect collective forces in the vertical dimension. Convergence studies for some of these parameters are presented in Appendix C. 


\subsection{Code Comparisons and Parametric Studies}

At ASTA, the bunch charge will be variable from a few pCs to several nCs. Some applications, e.g. the test of ILC subsystems for ILC bunch-parameters, calls for a $3.2 \mathrm{nC}$, while other experiments, such as high-brilliance X-ray generation via channeling radiation, require very low charge. It is therefore of interest to assess the performance of the $\mathrm{BC} 1$ over the anticipated range in charge. Following Refer-

ence [38], we introduce the transverse brightness $B_{\perp}=\frac{\hat{I}}{4 \pi^{2} \varepsilon_{x} \varepsilon_{y}}$. The charges used and their initial transverse normalized emittances are shown in Table 4.2.

Each of the simulation codes discussed in this dissertation use different methods of modeling the phase-space evolution of particles throughout the chicane, and account for collective effects in distinct ways. The most accurate simulations should be those that contain both space-charge effects and CSR effects. Both CSRTRACK's P2P model and ImpaCt-Z's SC+CSR model contain both CSR and SC effects, and each of these simulations has an advantage over the other. IMPACT-Z allows for a greater number of macroparticles, while CSRTRACK models transverse CSR effects, as well as the fact that CSR fields follow the bunch downstream of a dipoles exit. In this section, we compare the behavior between the various models for various energy chirps, $\mathcal{C}$, and bunch charges, $Q$.

For each of the bunch charges specified earlier, we performed scans of the LPS chirp. For IMPACT-Z's SC and SC+CSR models, as well as CSRTRACK's 1-D Projected model, we use the range of $\mathcal{C} \in[1.0,6.0]$, and 200k macroparticles. For CSRTRACK P2P model, we focus on a smaller range because of for the drastically increased execution-time required for these simulations, and use only 10k macroparticles with $\sigma_{B}=0.1 \sigma_{z}$. 
The bunch length downstream of BC1 depends on the initial distribution and the LPS chirp. Figure 4.8 shows the bunch lengths downstream of BC1 versus LPS chirp, for each of the four bunch charges and for both of IMPACT-Z's models. Shorter bunch lengths can be achieved for lower bunch charges and without CSR.

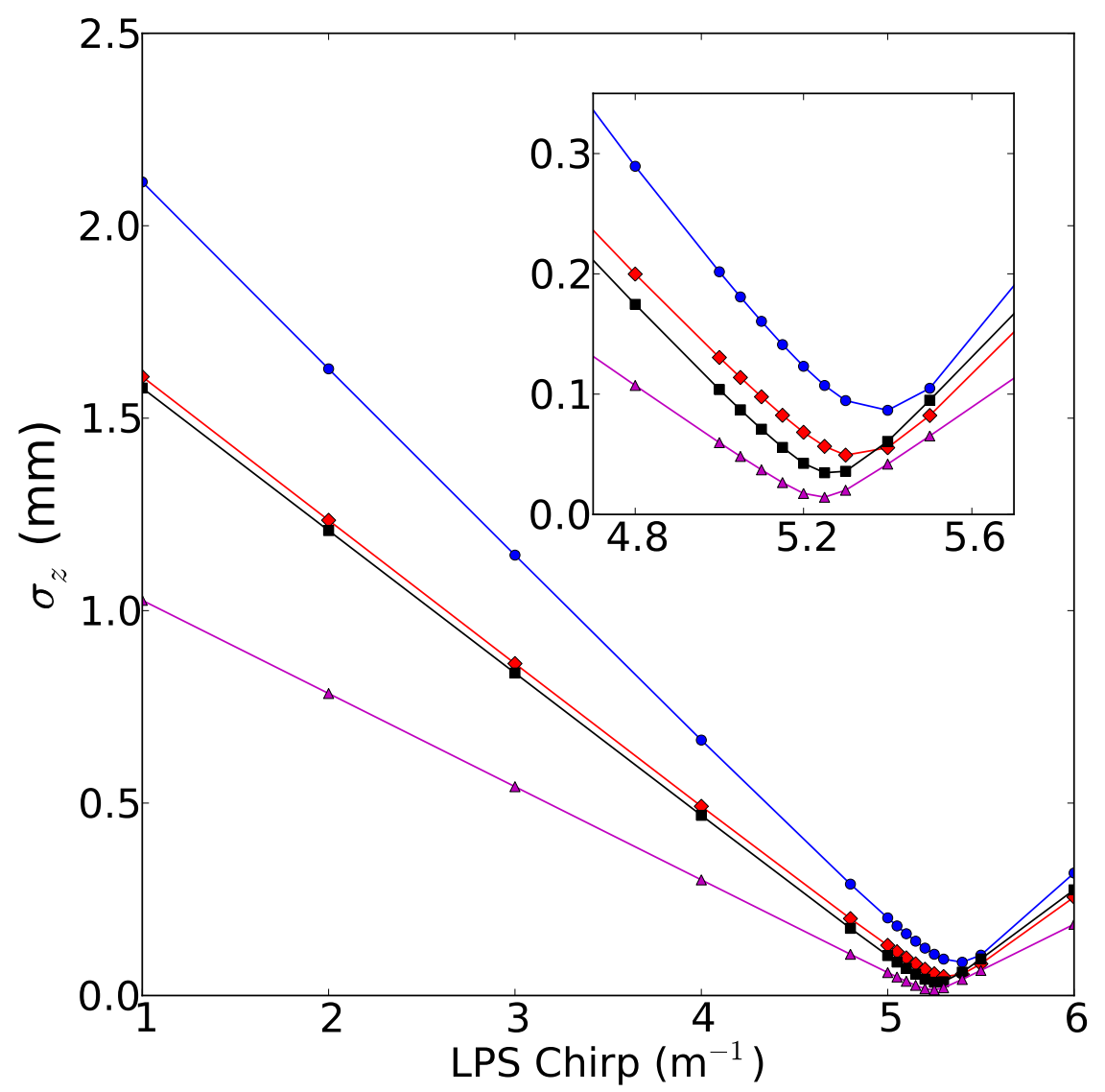

Figure 4.8: RMS bunch length $\sigma_{z}$ downstream of BC1 as a function of the LPS chirp for various bunch charges using IMPACT-Z's CSR+SC model model, for 3.2$\mathrm{nC}$ (blues), 1.0-nC (red), 250-pC (green), 20-pC (magenta) bunch charges. The inset plot corresponds to a close-up around chirp values that achieve minimum RMS bunch lengths.

At higher bunch charges, CSR has a greater contribution to emittance-dilution, while at low bunch charges, the space-charge effects are a greater contribution, as 
demonstrated in the reversal of the lines representing Impact-Z's SC-only model and CSRTRACK's 1DP model.

The agreement in emittance growth between the $\mathrm{SC}+\mathrm{CSR}$ and $\mathrm{P} 2 \mathrm{P}$ models is reasonable, and a visual comparison of the longitudinal phase-spaces shows many of the similar characteristics, shown in Figure 4.9. The CSRTRACK simulations are presumably the more accurate of the two, but IMPACT-Z's simulations take only a small fraction of the time, with vastly more particles.
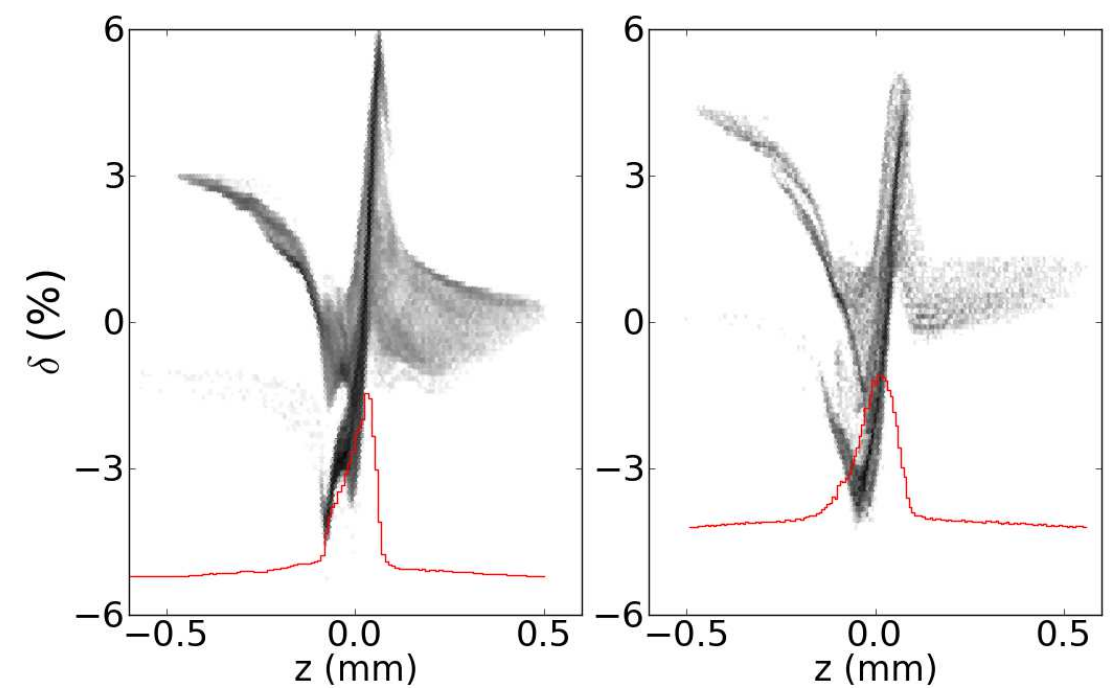

Figure 4.9: LPS at the end of the bunch compressor and associated longitudinal charge distribution (red trace) in arbitrary units, with IMPACT-Z using 200k particles (left) and CSRTRACK's P2P model with 30k particles and 10\% RMS subgaussians (right), for an initial chirp of $5.22 \mathrm{~m}^{-1}$ and bunch charge of $3.2 \mathrm{nC}$.

Simulations were performed for LPS chirps $\mathcal{C} \in[1.0,6.0] \mathrm{m}^{-1}$ with the bulk of the simulations performed around the maximum-compression value $\mathcal{C}=-1 / R_{56} \simeq$ $5.2 \mathrm{~m}^{-1}$, corresponding to enhanced collective effects.

The transverse dynamics through a bunch compressor are known to influence the relative emittance growth $[39,40]$. The initial values for the betatron functions were selected such that the beam experiences a waist between the third and fourth 
dipole [41]. Simulations performed with the 1DP CSRTRACK model also confirmed there is a set of horizontal C-S parameters that minimizes the bending-plane emittance growth as displayed in Figure 4.4. The upstream magnets were tuned to provide the incoming horizontal C-S parameter $\left(\beta_{x, i}, \alpha_{x, i}\right)=(8.0 \mathrm{~m}, 3.0)$ shown in Figure 4.5. Lastly, the final RMS bunch lengths as a function of the initial LPS chirp are given in Figure 4.8 as obtained using IMPACT-Z's SC+CSR model. In addition, there are slight variations in the bunch length between the four different models due to their collective effects inhibiting compression to varying degrees.

\subsection{Benchmarking of numerical models}

The beam dynamics simulations through $\mathrm{BC} 1$ were performed for several degrees of bunch compression (controlled with the LPS chirp) for the four cases of bunch charges that will be used at the ASTA. Four simulation algorithms were used as described earlier. Because of its computational effort, CSRTRACK's P2P model simulations were restrained to a smaller range of values for $\mathcal{C}$. The LPS 1.0$\mathrm{m}$ downstream of the $\mathrm{B} 4$ dipole simulated with IMPACT-Z ( $\mathrm{SC}+$ one-dimensional CSR models) and CSRTRACK (P2P model) are shown in Figure $4.10[(\mathrm{a})-(\mathrm{d})]$ and [(e)-(f)] respectively for the four charges listed in Table 4.2.

Despite the vastly different algorithms used by these two programs, the LPS distributions displayed very similar distortions including those at the small-scale levels. Figure 4.11 summarizes the evolution of peak current as a function of the initial LPS chirp for the four numerical models. Likewise the longitudinal emittances computed with IMPACT-Z (SC+CSR) and CSRTRACK (P2P) for the case of maximum compression are in decent agreement; see Table 4.4. 

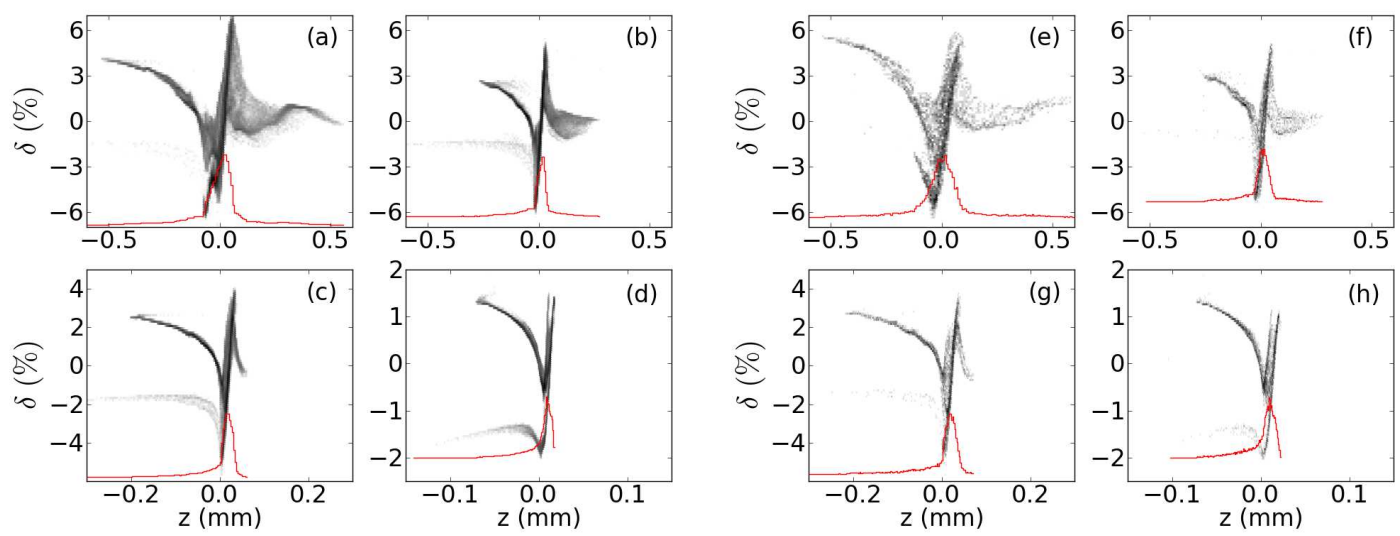

Figure 4.10: LPS at BC1 exit for IMPACT-Z's (a-d) and CSRTRACK's (e-h) 3D models, for 3.2-nC (a,e), 1-nC (b,f), 250-pC (c,g), and 20-pC (d,h) bunch charges, zoomed in to show details, for $\mathcal{C}=5.2 \mathrm{~m}^{-1}$. (black line) Longitudinal current projection, with arbitrary scale and offset. Note that the horizontal and vertical axis ranges are different for each plot. The ordinates $z>0$ correspond to the head of the bunch.

The transverse-emittance after compression is shown in Figure 4.12. CsRTRACK's P2P model consistently yields the greatest emittance growth, followed by IMPACT-Z's SC+CSR model, as these are the only two models that account for both SC and CSR effects. However, the P2P model also includes transverse CSR forces and a more elaborate model for longitudinal CSR. Our previous study [42] showed that the influences on final emittance from using too-few macroparticles as well

Table 4.4: Final normalized longitudinal $\varepsilon_{z}$ at maximum compression $\left(\mathcal{C}=5.2 \mathrm{~m}^{-1}\right)$ simulated with impact-z (SC+CSR) and CSRTRACK (P2P).

\begin{tabular}{ccc}
\hline & $\begin{array}{c}\text { IMPACT-Z } \\
\text { SC+CSR }\end{array}$ & $\begin{array}{c}\text { CSRTRACK } \\
\text { P2P }\end{array}$ \\
$\mathrm{Q}(\mathrm{nC})$ & $\varepsilon_{z}(\mu \mathrm{m})$ & $\varepsilon_{z}(\mu \mathrm{m})$ \\
\hline \hline 3.2 & 267 & 261 \\
1.0 & 118 & 105 \\
0.250 & 61.5 & 57.8 \\
0.020 & 10.5 & 11.6 \\
\hline
\end{tabular}



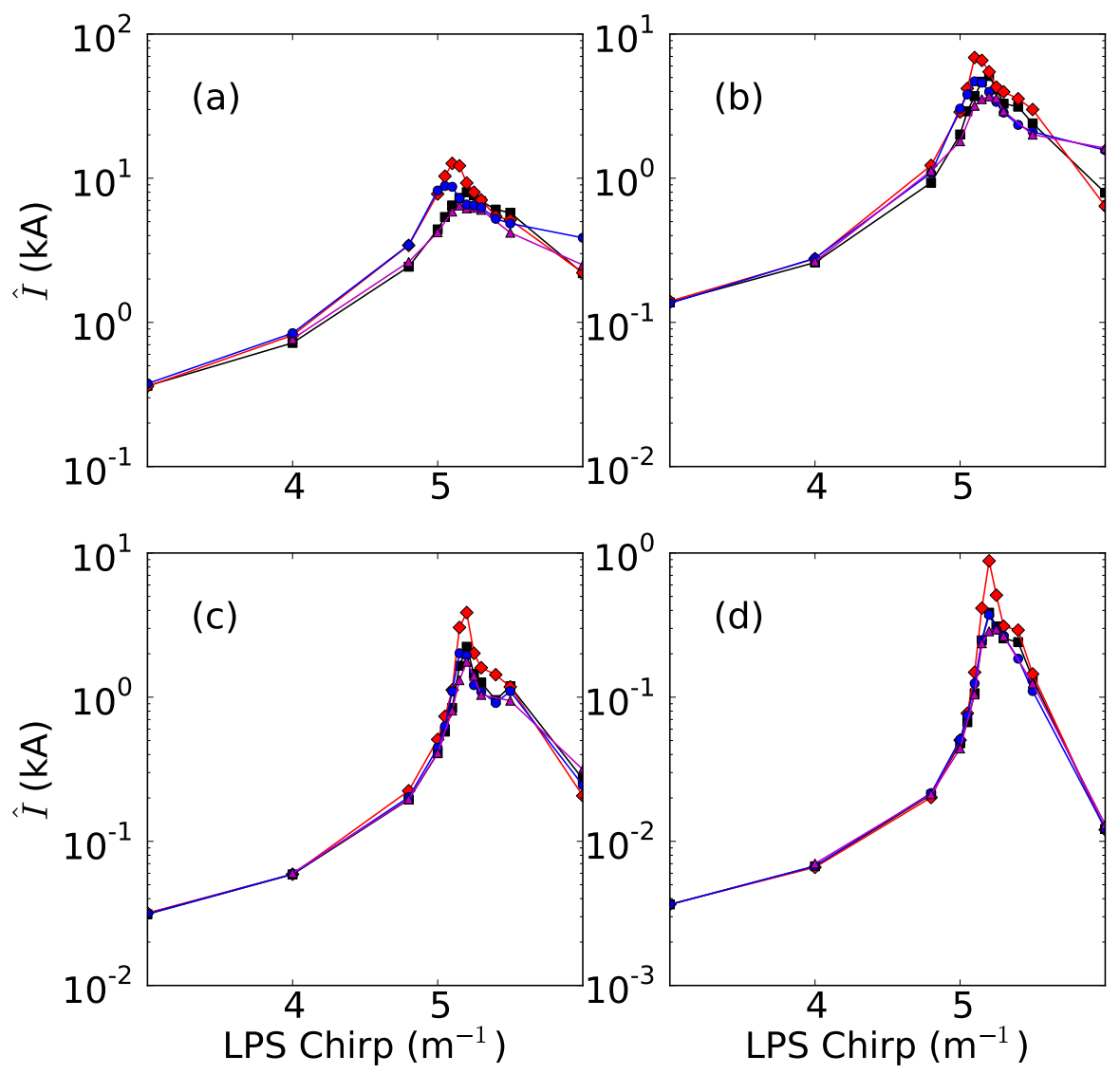

Figure 4.11: Peak currents $\hat{I}$ versus energy chirp for IMPACT-Z's SC+CSR (squares), IMPACT-Z's SC (circles), CSRTRACK's 1DP (diamonds), and CSRTRACK's P2P (triangles) models, for 3.2-nC (a), 1.0-nC (b), 250-pC (c), and 20-pC (d) bunch charges.

as from the randomization in the down-sampling of initial distributions were both much smaller than the discrepancy between the SC+CSR and P2P models. The emittance growth observed from CSRTRACK's 1D and IMPACT-Z's SC-only model indicates that CSR accounts for most of the emittance dilution at higher charge. For the low-charge simulations $(Q=250$ and $20 \mathrm{pC})$ the relative importance is reversed, with $\mathrm{SC}$ contributing more to the emittance degradation than CSR. 
Of the models presented here, only IMPACT-Z's include SC in both the vertical and the horizontal planes, and are shown in Figure 4.13. As vertical emittance growth is entirely the result of SC, the inclusion of IMPACT-Z's CSR model reduces vertical emittance growth due to the reduced compression.

Finally, the evolution of the slice parameters during compression were explored. For this analysis the beam is divided into axial slices of equal longitudinal length $\delta z=20 \mu \mathrm{m}$. A statistical analysis on the population contributing to each slice was performed to yield the slice emittances, energy spread and peak current. A comparison of the slice bending-plane emittance and energy spread between IMPACT$\mathrm{Z}$ and CSRTRACK's P2P model of slice-emittance as the chirp is varied appears in Figure 4.14. The level of agreement between the two programs is of the same order as what observed for the bunch parameters. Figure 4.15 summarizes the evolution of the slice horizontal emittance in the slice with the highest peak current within the bunch. When the beam is greatly over-compressed $\left(\mathcal{C}=6.0 \mathrm{~m}^{-1}\right)$, the LPS may be double-peaked, and the transverse brightness as defined here may not be an appropriate measure of the bunch's utility. The curve demonstrates how little slice emittance growth occurs for partial compression.

\subsection{Expected beam dilution and trade-offs}

A large number of accelerator applications require beams with high-peak-currents and low-transverse-emittances. These requirements conflict with each other as collective effects, which dilute the beam's phase space and emittances, increase with peak current. A commonly used figure of merit is peak transverse brightness $B_{\perp} \equiv \frac{\hat{I}}{4 \pi^{2} \varepsilon_{x} \varepsilon_{y}}$ [38]. Figure 4.16 summarize the evolution of $B_{\perp}$ as function of the LPS 
chirp for the four cases of bunch charges. The figure combines the data provided in Figure 4.11 and Figure 4.12. Despite the lower-charge bunch result in smaller peak current at lower charges (see Figure 4.11), the transverse brightness increases with lower bunch charges. The main factor at play in this reduction is the lower initial transverse emittances, and more importantly, the reduced dilution of the transverse emittances during compression in $\mathrm{BC} 1$ due to the weaker collective effects (CSR and $\mathrm{SC})$.

In addition we note that the maximum achieved transverse brightness does not necessarily occur at maximum compression. This is due to the larger peak currents at maximum compression that drive collective effects, wherein the relative emittance growth driven by collective effects is greater than the relative increase in peak current, a trade-off similar to that of going down to lower bunch charges. A summary of the achieved maximum value of $B_{\perp}$ appears in Figure 4.17.

The trade-off between obtained peak current and $\varepsilon_{x}$ is shown in Figure 4.18; only data associated to LPS chirp up to maximum compression at $\mathcal{C} \leq 5.2 \mathrm{~m}^{-1}$ are displayed, as over-compression results in lower peak currents with generally larger emittance dilutions.

The simulation data points to several conclusions about the parametric trade-offs that must be considered. First, the emittance growth, particularly the slice emittance, is greatly reduced at lower degrees of compression, particularly $\mathcal{C}<4 \mathrm{~m}^{-1}$, which corresponds to compression to around one-third of the initial bunch length. Second, using lower bunch-charges is preferred for experiments that require high transverse brightnesses, due to the lower emittance growth from collective effects justifying the lowered peak current. For the 20-pC bunch charge, the regime with $\mathcal{C}<4 \mathrm{~m}^{-1}$ results in horizontal emittance growth. Each of the different simulation codes is applicable for different parts of the beamline. Astra has the most accurate 
SC modeling, but is also the most computationally intensive, offers little benefit in tracking at the beam energy post-CAV1 and CAV2, and incapable of simulating bunch compressors. CsRTRACK is specifically designed to model bending magnets, and is thus inappropriate for tracking at low energies, through basic beam transport, or for low-energy simulations near the photocathode. IMPACT-Z is the most computationally efficient of the three simulations, but is inaccurate at very low energies, such as prior to CAV1 and CAV2. For these reasons, we use ASTRA.wth that is under $10 \%$ of the initial horizontal emittance, regardless of which of the simulation codes is used.

\subsection{High-Energy Limit}

To help demonstrate the differences between the SC and CSR models implemented in the codes, we increase the energy of the particles to $400 \mathrm{MeV}$ while keeping the emittances the same, and perform the same scan of LPS chirp as described earlier with IMPACT-Z's SC+CSR and CSRTRACK's P2P model using the same chicane parameters.

As transverse SC effects are proportional to $\gamma^{-2}$, SC effects are almost completely suppressed at $400 \mathrm{MeV}$, with IMPACT-Z's SC model showing near-zero emittance growth. The other three simulations show varying degrees of emittance growth due to the different models used by each one- IMPACT-Z's version of the Saldin method, CSRTRACK's 1DP's kernel method, and CSRTRACK's multidimensional model. Due to the suppression of SC effects, we can infer that the resulting discrepancies are only functions of the different SC models. While CSRTRACK's 1-D Projected and Particle-to-Particle models are in good agreement, the Saldin model 
for CSR implemented in IMPACT-Z appears to be significantly weaker than the energy-dependent model used in CSRTRACK. This may be a result of both of CSRTRACK's models including some transverse forces, which are absent from the Saldin model.

\subsection{Bunch Shaping}

Over-compression may allow one to create current profiles that are well matched to some potential AARD applications. For instance, linearly-ramped current profiles can be used to explore beam-driven acceleration methods with an improved transformer ratio $[43,44,6]$. Likewise, a double-peaked distribution could prove useful to investigate and optimize the longitudinal beam dynamics along the ASTA accelerator. Such a double-peaked distribution could also support the test of beam-driven acceleration where the first bunch generates wakefields with sub-mm wavelengths and a second, lower-charge "witness" bunch experiences the accelerating fields produced by the first bunch. Such a distribution turns out to be readily achievable with $\mathrm{BC} 1$, as shown in Figure 4.21. The distribution displayed in Figure 4.21 is obtained by over-compressing a $3.2-\mathrm{nC}$ bunch with $\mathcal{C}=5.50 \mathrm{~m}^{-1}$. The obtained bunch separation $\Delta z \simeq 0.3 \mathrm{~mm}$ is consistent with the period of wakefields produced in dense plasma [45] or dielectric-wakefield waveguides [7].

\subsection{High peak current production}

Despite their relatively poor transverse emittance, fully compressed bunches could be employed to generate copious amounts of radiation via a variety of elec- 
tromagnetic processes. The spectral-angular fluence emitted by a bunch of $N \gg 1$ electrons from any electromagnetic process is related to the single-electron spectral fluence, $\left.\frac{d^{2} W}{d \omega d \Omega}\right|_{1}$, via

$$
\left.\left.\frac{d^{2} W}{d \omega d \Omega}\right|_{N} \simeq \frac{d^{2} W}{d \Omega d \omega}\right|_{1}\left[N+N^{2}|S(\omega)|^{2}\right]
$$

where $\omega \equiv 2 \pi f$ ( $f$ is the frequency) and $S(\omega)$, the bunch form factor (BFF), is the intensity-normalized Fourier transform of the normalized charge distribution $S(t)$ [46]. The former equation assumes the bunch can be approximated as a line charge distribution and is practically valid as long as the rms bunch duration $\sigma_{t}$ and transverse size $\sigma_{\perp}$ satisfy $\sigma_{\perp} \ll c \sigma_{t} / \gamma$ where $\gamma$ is the Lorentz factor and $c$ is the velocity of light. When the BFF approaches unity, $\left.\frac{d^{2} W}{d \omega d \Omega}\right|_{N} \propto N^{2}$ and the radiation is termed "coherent radiation".

At ASTA the availability of a superconducting linac coupled with a non-interceptive radiation-generation mechanism (e.g., diffraction radiation [47],[48]) could lead to the production of single-cycle $\mathrm{THz}$ pulses repeated at $3 \mathrm{MHz}$ over 1-ms. As an example we consider the worst-case scenario of a fully compressed 3.2-nC bunch; the dependency of the BFF over frequency appears in Figure 4.22 (left plot). The BFF starts to take off at frequency lower than $f \simeq 1 \mathrm{THz}$, thereby supporting the generation of coherently-enhanced radiation at these frequencies. A limitation might come from the large transverse emittance that would prevent the beam to be focused to a transverse spot RMS size below the required $\sigma_{\perp} / \gamma$ value. However, a statistical analysis indicates that the central part of the beam containing approximately $15 \%$ of the beam population (or 500-pC out of the original 3.2-nC bunch) has emittances below $10 \mu \mathrm{m}$ resulting in beam $\sigma_{\perp} \leq 100 \mu \mathrm{m}$; see Figure 4.22 (right plot). It should be pointed out that the lower-charge cases investigated in the previous section would 
result in shorter pulses with associated BFFs that contain higher-frequency content (see also Figure 4.8).

\subsection{Compressed flat-beam generation}

An important asset of the ASTA photoinjector is its capability to generate beams with high-transverse emittance ratios known as flat beams. Immersing the photocathode in a magnetic field introduces a canonical angular momentum $\langle L\rangle=e B_{0} \sigma_{c}^{2}$, with $B_{0}$ the magnetic field on the photocathode surface, and $\sigma_{c}$ the RMS transverse size of the drive-laser spot on the photocathode [13]. As the beam exits the solenoidal field provided by lenses L1 and L2, the angular momentum is purely kinetic, resulting in a beam coupled in the two transverse planes. Three skew quadrupoles in the beamline can apply the torque necessary to cancel the angular momentum $[14,15]$. As a result, the final beam's transverse emittance partition is given by

$$
\left(\varepsilon_{x, i}, \varepsilon_{y, i}\right)=\left(\frac{\varepsilon_{u}^{2}}{2 \beta \gamma \mathcal{L}}, 2 \beta \gamma \mathcal{L}\right),
$$

where $\varepsilon_{u}$ is the normalized uncorrelated emittance of the magnetized beam prior to the transformer, $\beta$ and $\gamma$ the Lorentz factors, $\mathcal{L} \equiv\langle L\rangle / 2 p_{z}$, and $p_{z}$ is the longitudinal momentum. Note that the product $\varepsilon_{x, i} \varepsilon_{y, i}=\left(\varepsilon_{u}\right)^{2}$. If compressed, these flat beams may have applications in Smith-Purcell FELs [49] or for beam-driven acceleration techniques using asymmetric structures [50]. It may also be possible to mitigate the emittance growth in $\mathrm{BC} 1$ by having a beam that is wide in the direction of the chicane bend.

In this section, we explore the behavior of flat beams in the low-energy bunch compressor at ASTA, for different initial emittance ratios $\rho \equiv \varepsilon_{x, i} / \varepsilon_{y, i}$. In order to 
Table 4.5: Initial parameters for different aspect ratios.

\begin{tabular}{ccc}
\hline Aspect Ratio & $\varepsilon_{x i}(\mu \mathrm{m})$ & $\varepsilon_{y i}(\mu \mathrm{m})$ \\
\hline \hline 1 & 5 & 5 \\
5 & 11 & 2.2 \\
25 & 25 & 1 \\
50 & 35 & 0.70 \\
100 & 50 & 0.5 \\
200 & 71 & 0.35 \\
400 & 100 & 0.25 \\
\hline
\end{tabular}

produce these bunches, we took the 3.2-nC bunch presented earlier and numerically scaled the macroparticle coordinates to produce the desired transverse emittance ratios while constraining the product $\varepsilon_{x, i} \varepsilon_{y, i}=5^{2} \mu \mathrm{m}^{2}$. Specific values for the partitioned emittances are shown in Table. 4.5.

Due to the large transverse aspect ratio of the bunches, the criterion given in Equation C.5 is generally not satisfied and it is therefore anticipated that the projected CSR model is inadequate; thus we use CSRTRACK's P2P model to simulate the flat beams and neglect the 1DP model. The parameters used for flat beam simulations follow those used in the previous section, with the exception of the macroparticle horizontal size used in the CSRTRACK P2P model. Due to the much greater transverse dimension we set $\sigma_{h}=0.2 \mathrm{~mm}$. In addition, IMPACT-Z SC+CSR simulations were also performed to evaluate the emittance growth in the vertical plane. The simulated emittance growth is shown in Figure 4.25 for a 3.2-nC bunch with an initial LPS chirp of $\mathcal{C}=5.2 \mathrm{~m}^{-1}$. As expected, the relative emittance dilution is reduced as the initial emittance ratio $\rho$ increases. The agreement between CSRTRACK and IMPACT-Z for the bending-plane emittance dilution is remarkable (within $\sim 30 \%$ ) given the large transverse horizontal beam sizes. IMPACT-Z predicts that the vertical emittance increases by a factor of 1.5 to 1.8 over the range 
of considered initial emittance ratios $\rho \in[1,500]$. The four-dimensional transverse emittance growth $\varepsilon_{4} \equiv \sqrt{\epsilon_{x} \epsilon_{y}}$ is mitigated for the larger initial flat-beam emittance ratios. As these simulations were performed by numerically stretching the same initial 3.2-nC bunches used for the simulations presented elsewhere in this paper, the further investigation of the compression of more realistic flat beam distributions is warranted.

\subsection{Double-bunch generation}

The production of shaped electron bunches has a large number of applications including the investigation of wakefield and beam-driven acceleration techniques. Operating the low energy bunch compressor with LPS chirp $\mathcal{C}>5.5 \mathrm{~m}^{-1}$ leads to over compression and results in a structured longitudinal charge distribution. Figure 4.26 confirms, for the case of $Q=3.2 \mathrm{nC}$, that a bi-modal distribution could be generated with a separation between its peaks $(\sim 300 \mu \mathrm{m})$ consistent with requirements from beam-driven acceleration such as plasma-wakefield and dielectricwakefield acceleration techniques. In addition the distance between the peaks could be controlled to some degree by slight changes over the initial LPS chirp. The fullbunch and slice-at-peak-current horizontal emittance at $\mathcal{C} \sim 5.5 \mathrm{~m}^{-1}$ are 67.0 and $75.1 \mu \mathrm{m}$, respectively, compared to 106 and $107 \mu \mathrm{m}$ for the maximum-compression case $\left(\mathcal{C} \sim 5.2 \mathrm{~m}^{-1}\right)$. These bending-plane normalized emittances of $\sim 75 \mu \mathrm{m}$ can still be focused to a sub-mm or sub-100- $\mu \mathrm{m}$ transverse spot size at respectively $\sim 40 \mathrm{MeV}$ and $\sim 250 \mathrm{MeV}$ (the latter energy corresponds to acceleration of the 40 $\mathrm{MeV}$ beam into one of ASTA accelerating cryomodules). Detailed investigations of 
further acceleration of these types of exotic bunches through the ASTA lattice will need to be performed.

\subsection{BC1 Summary}

Several simulation codes and models were tested for the design parameters of a low-energy bunch compressor at the ASTA facility, over a wide range of simulation

parameters, including bin-size, number of particles, energy chirp, and length of Gaussian sub-bunches, and across various models in IMPACT-Z and CSRTRACK. There is reasonable agreement between the two SC+CSR models, and their final LPSs show much of the same behavior. Their accuracy will be compared against the early experimental results when the ASTA facility begins operation this year.

The large emittance growth and subsequent loss of brightness at high compression and high bunch charges indicates that user experiments that require highbrightness beams should explore using lower bunch charges and/or compression should be staged with a second bunch compressor at a higher energy after subsequent acceleration in the cryomodules. 

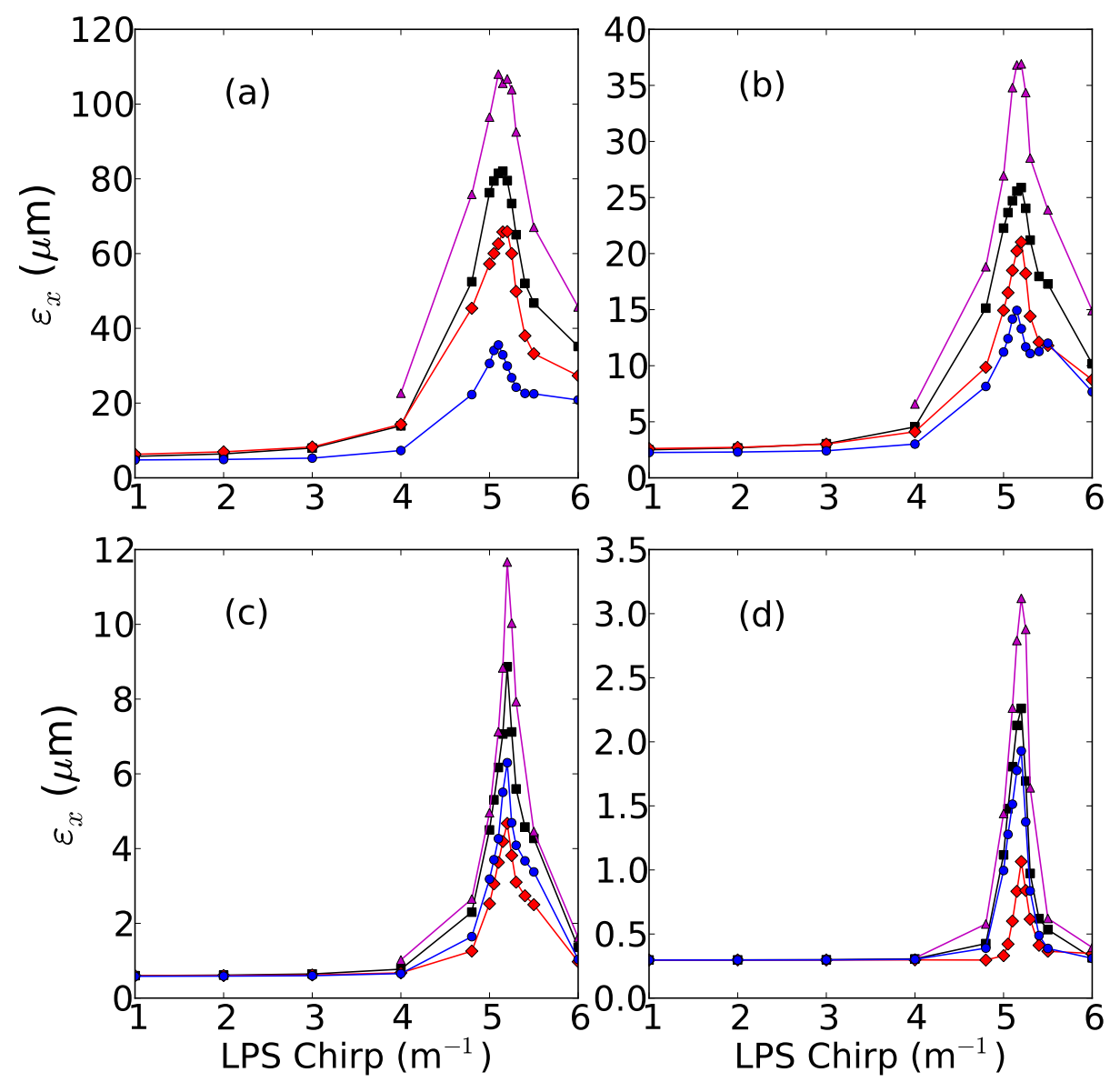

Figure 4.12: Final horizontal emittances for each of the different bunch charges with IMPACT-Z's SC-only model (blue), CSRTRACK's 1D CSR-model (red), IMPACT-Z's $\mathrm{SC}+\mathrm{CSR}$ model (green), and CSRTRACK's P2P model (magenta), for 3.2-nC (a), 1-nC (b), 250-pC (c), and 20-pC (d) bunch charges. 

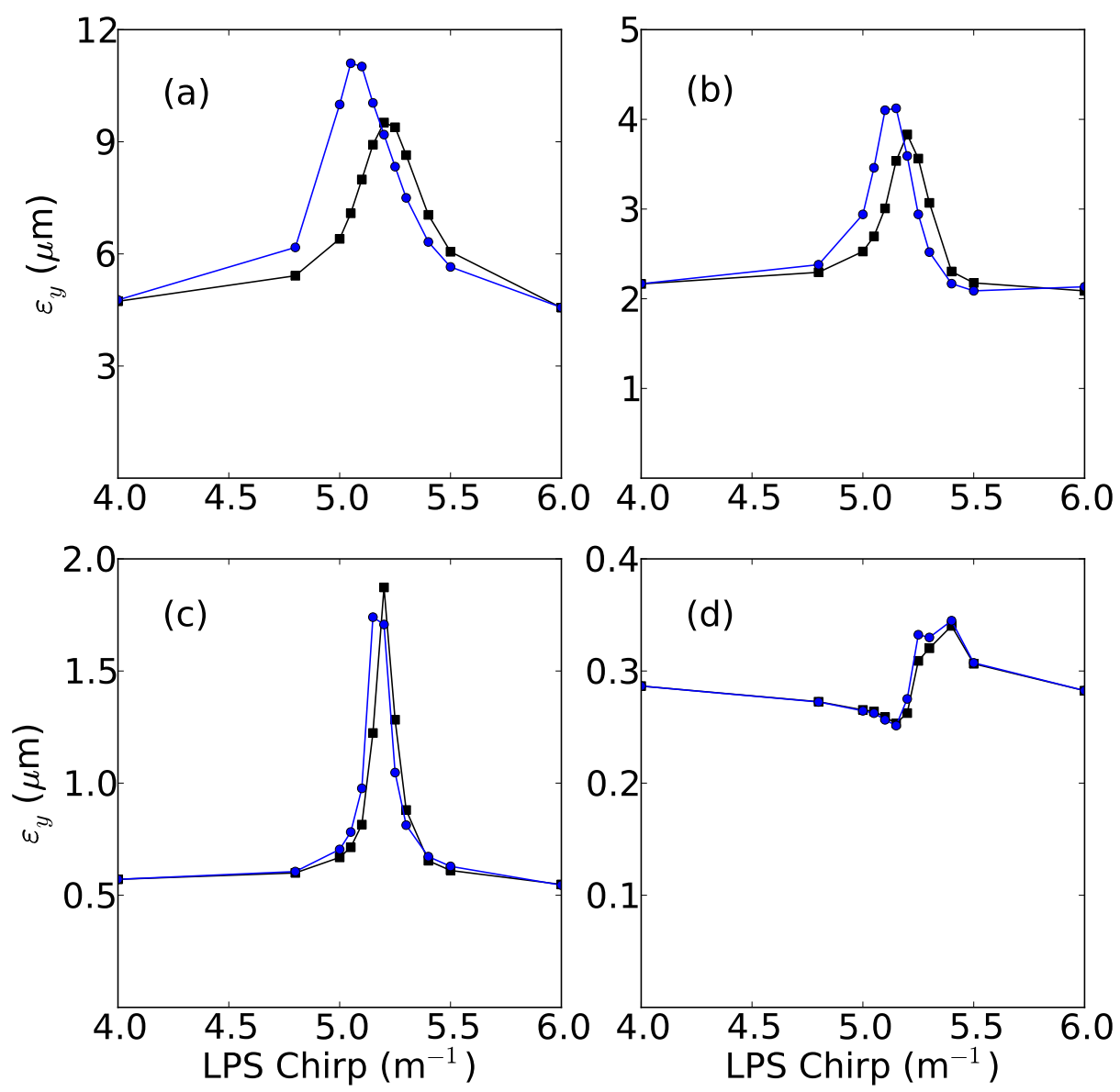

Figure 4.13: Final vertical emittances for each of the different bunch charges with IMPACT-Z's SC-only model (circles) and IMPACT-Z's SC+CSR model (squares), for 3.2-nC (a), 1-nC (b), 250-pC (c), and 20-pC (d) bunch charges. CsrTrack does not compute vertical forces, so the emittance remains roughly constant along the bunch compressor. 

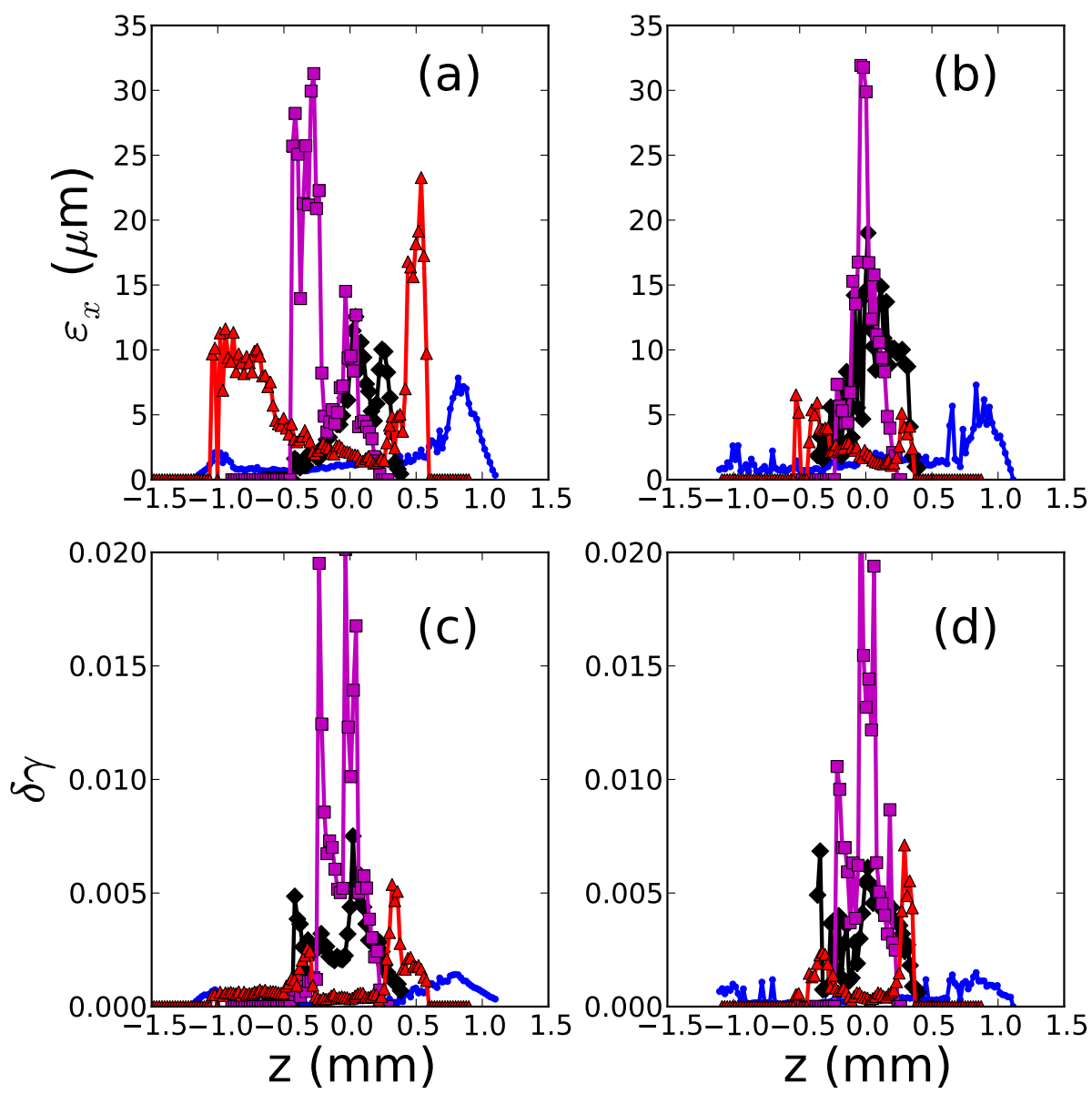

Figure 4.14: Example of final normalized transverse slice emittances (top row) and energy spread (bottom row) evolution within a 1-nC bunch for four cases of compression $\mathcal{C}=4.0,5.0,5.25$, and $6.0 \mathrm{~m}^{-1}$ respectively shown as dots, diamonds, triangles and square data points). Plots (a) and (c) correspond to IMPACT-Z simulations while plots (b) and (d) are results from CSRTRACK's P2P model. Emittance and energy spread values associated to slices that contain too-few number of macroparticle for meaningful statistical analysis are set to zero. The heads and tails of the bunches are sparsely populated (see Figure 4.10 for reference), particularly for the $\mathrm{P} 2 \mathrm{P}$ simulations which use only $5 \%$ of the number of particles used in the IMPACT-Z simulations. 

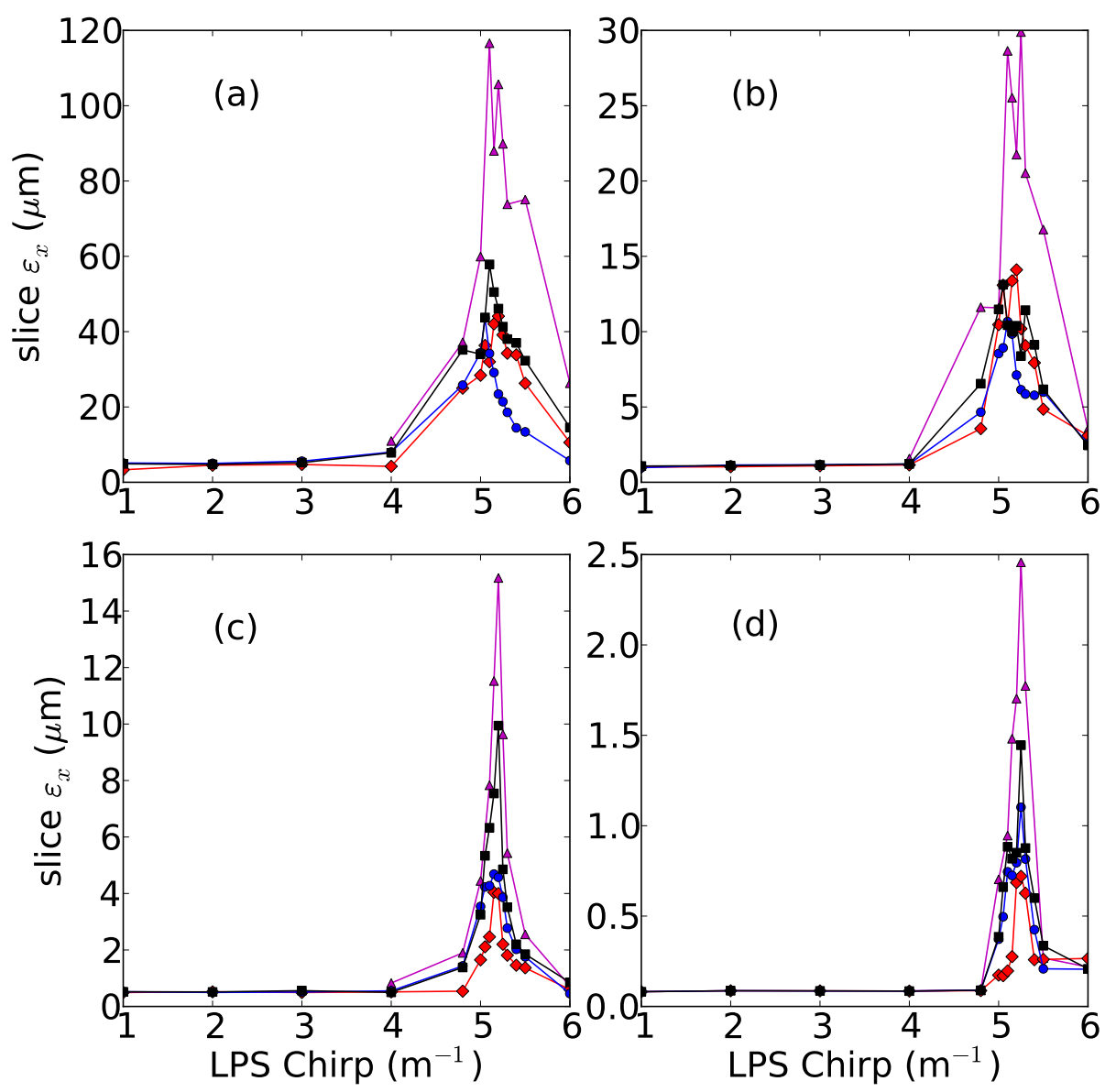

Figure 4.15: Final normalized transverse slice emittances $\varepsilon_{x, M}$ in the slice with the highest peak current computed with IMPACT-Z's SC-only model (circles), CSRTRACK's 1D CSR-model (diamonds), and IMPACT-Z's SC+CSR model (squares), and CSRTRACK's P2P model (triangles), for 3.2-nC (a), 1-nC (b), 250-pC (c), and 20-pC (d) bunch charges. 

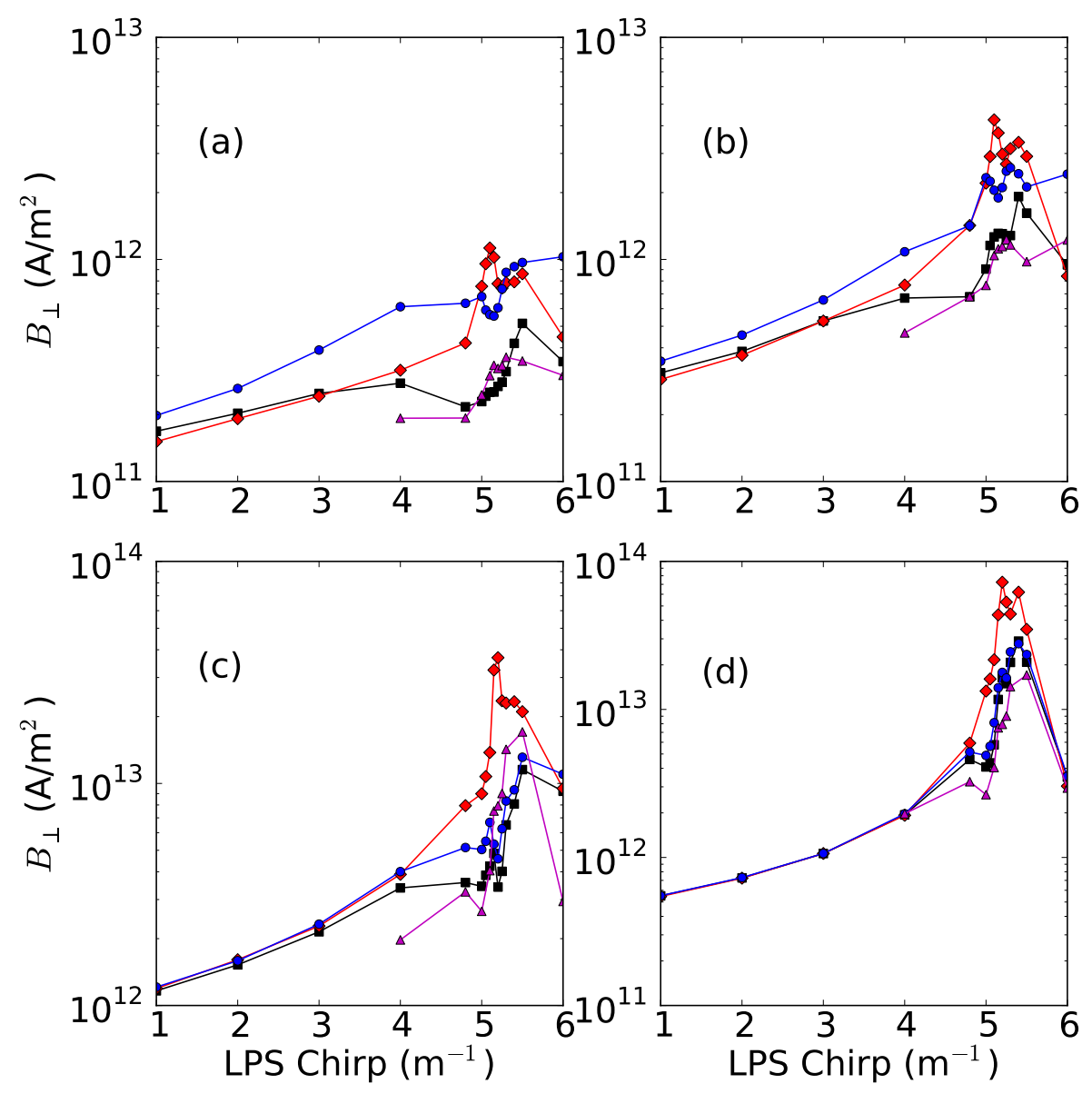

Figure 4.16: Peak transverse brightness $B_{\perp}=\frac{\hat{I}}{4 \pi^{2} \varepsilon_{x} \varepsilon_{y}}$ versus energy chirp for IMPACTZ's SC+CSR (squares), IMPACT-Z's SC (circles), CSRTRACK's 1DP (diamonds), and CSRTRACK's P2P (triangles) models, for 3.2-nC (a), 1.0-nC (b), 250-pC (c), and 20-pC (d) bunch charges. 


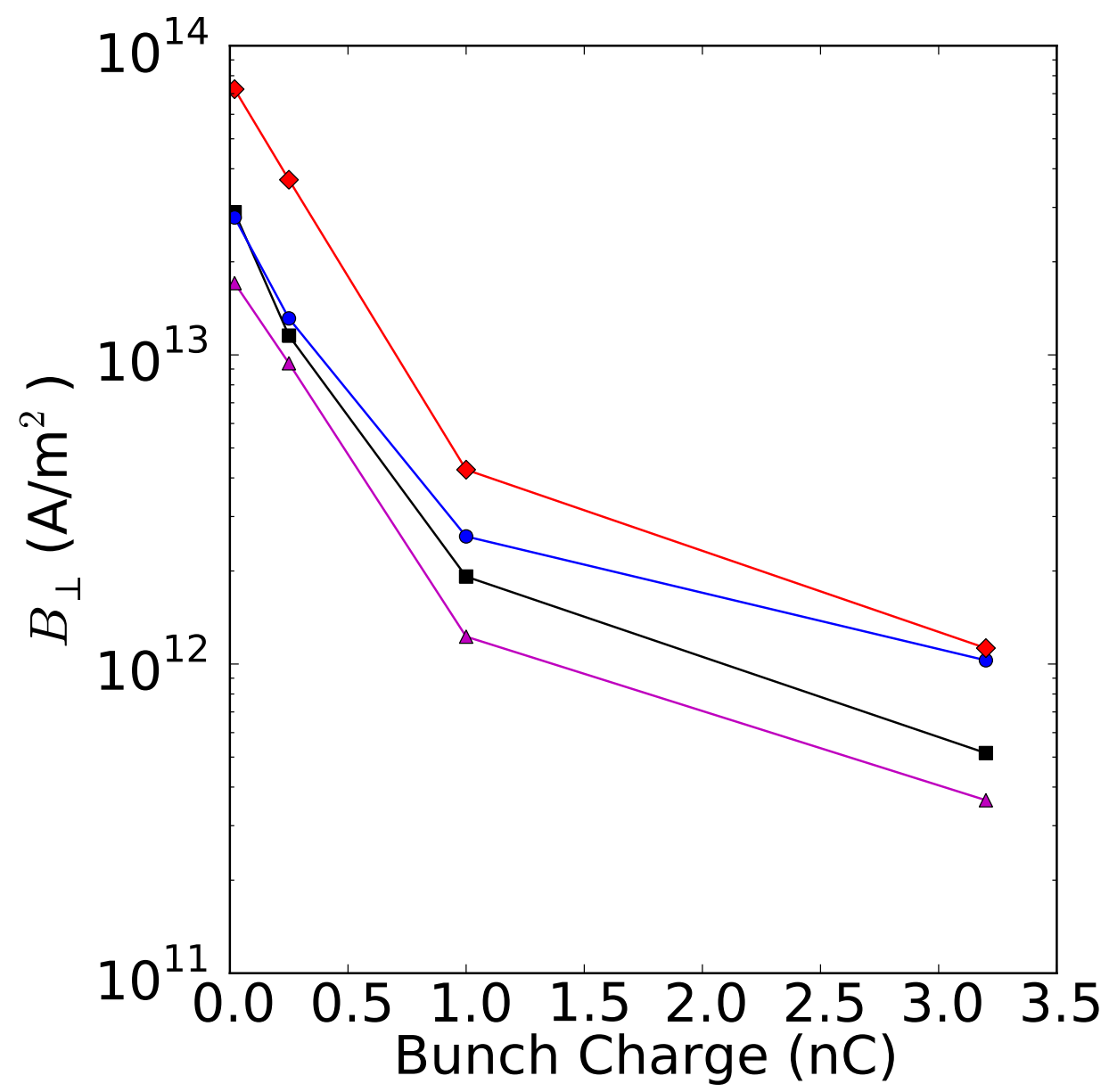

Figure 4.17: Maximum peak transverse brightness $B_{\perp}=\frac{\hat{I}}{4 \pi^{2} \varepsilon_{x} \varepsilon_{y}}$ versus bunch charge for IMPACT-Z's SC+CSR (squares), IMPACT-Z's SC (circles), CSRTRACK's 1DP (diamonds), and CSRTRACK's P2P (triangles) models. Each data point is a maximum from each line in Figure 4.16. 

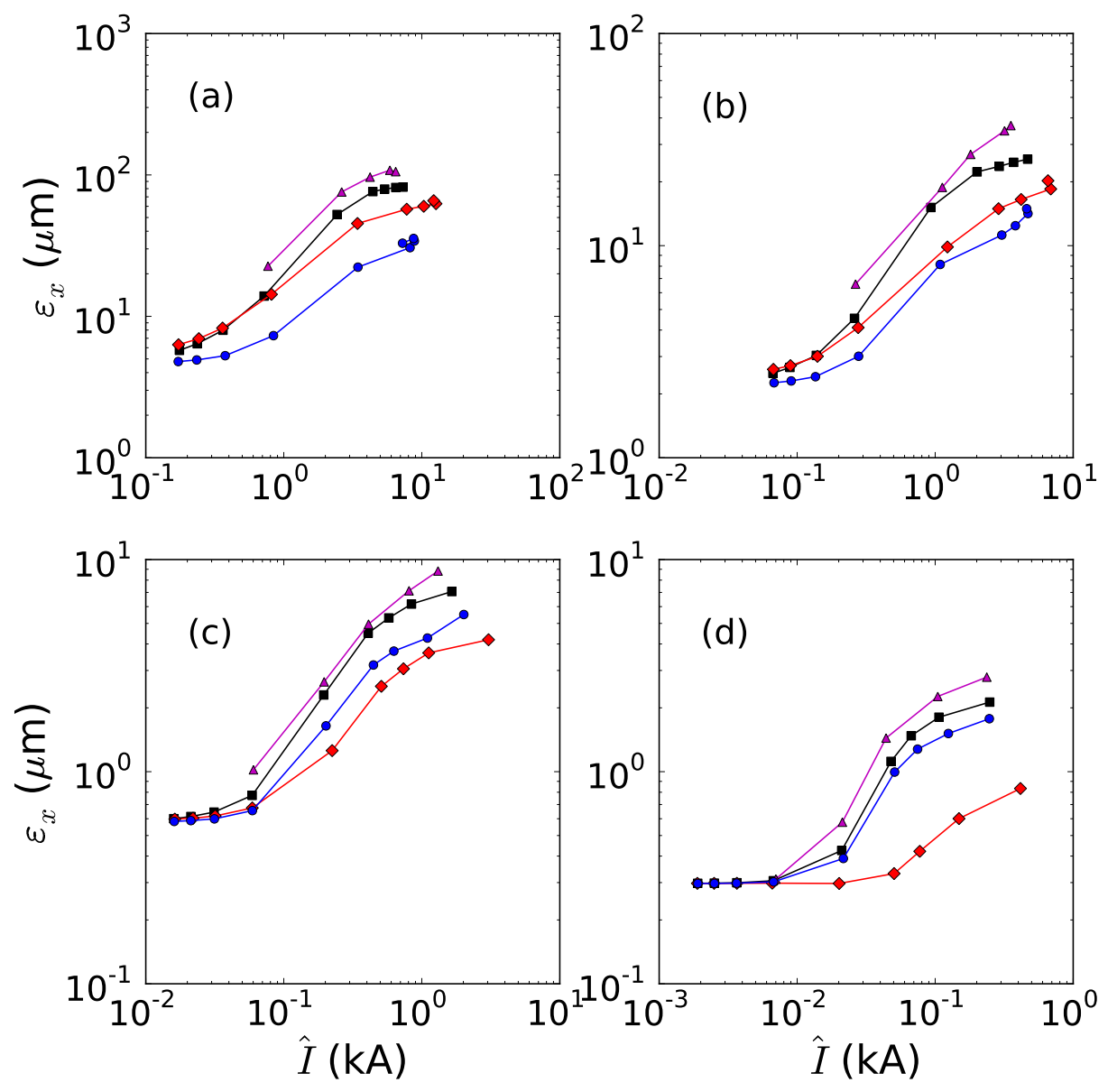

Figure 4.18: Final normalized transverse emittances $\varepsilon_{x}$ versus peak currents $\hat{I}$ for LPS chirps using IMPACT-Z's SC+CSR (squares), IMPACT-Z's SC (circles), CSRTRACK's 1DP (diamonds), and CSRTRACK's P2P (triangles) models, for 3.2-nC (a), 1.0-nC (b), 250-pC (c), and 20-pC (d) bunch charges. Only data corresponding to chirp values $\mathcal{C} \in[1.0,5.2] \mathrm{m}^{-1}$ are displayed. 

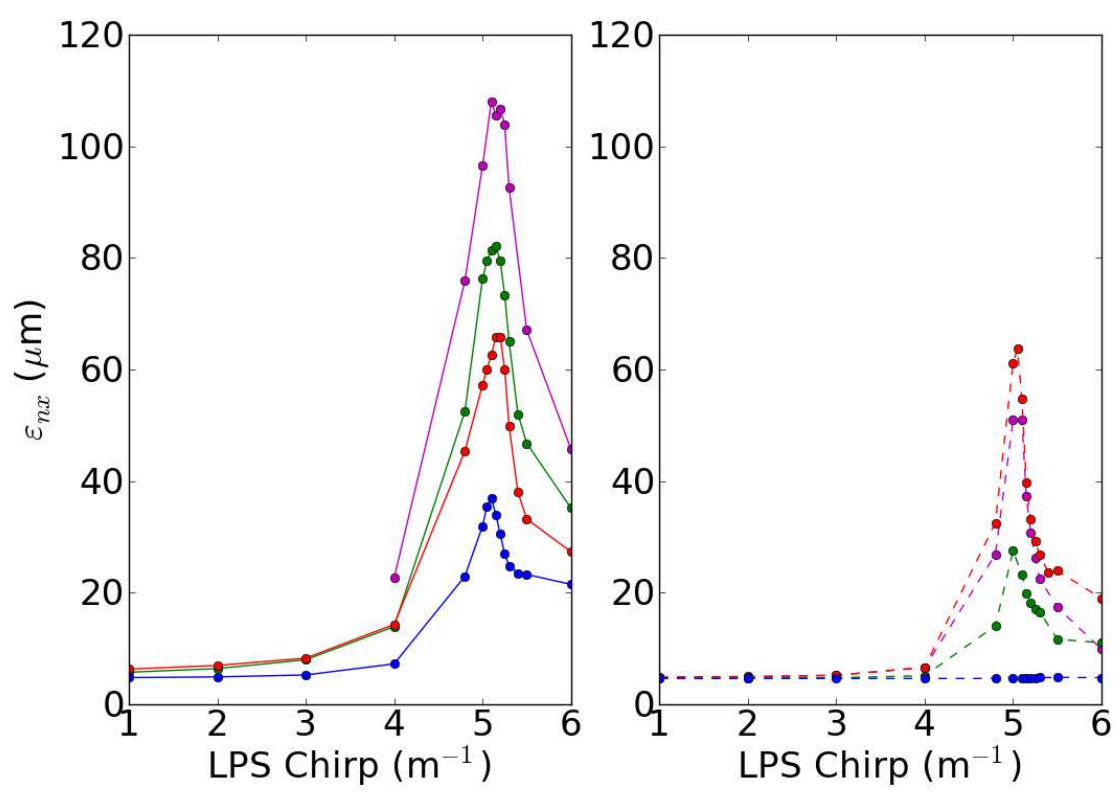

Figure 4.19: Final transverse emittance $\varepsilon_{x}(\mu \mathrm{m})$ versus LPS chirp using IMPACTZ's SC+CSR (green), ImpaCT-Z's SC (blue), CSRTRACK's 1DP (red), and CSRTRACK's P2P (magenta) models, at 38.6 MeV (left) and $400 \mathrm{MeV}$ (right). SC-driven emittance growth is almost totally mitigated at such high energies, while the three models of CSR offer varying emittance growths, with reasonable agreement.
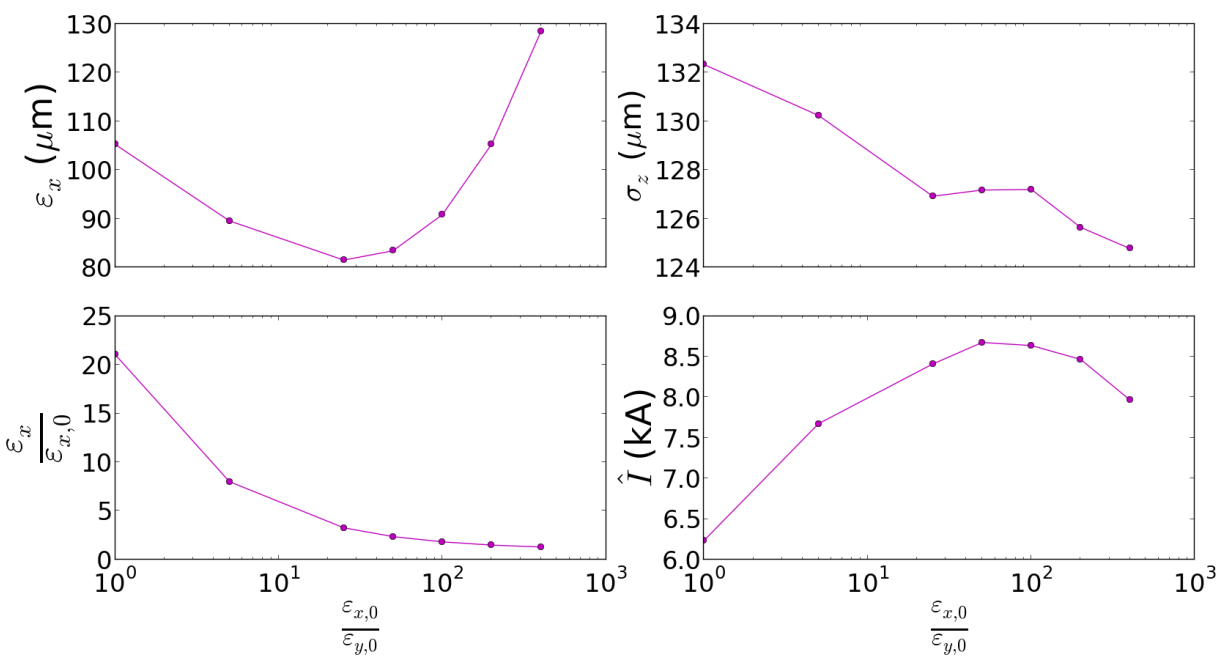

Figure 4.20: Final transverse emittances $\varepsilon_{x}$, relative emittance growths $\frac{\varepsilon_{x}}{\varepsilon_{y, 0}}$, bunch lengths $\sigma_{z}$, and peak currents at the exit of $\mathrm{BC} 1$ as functions of the initial aspect ratio, $\frac{\varepsilon_{x, 0}}{\varepsilon_{y, 0}}$ 

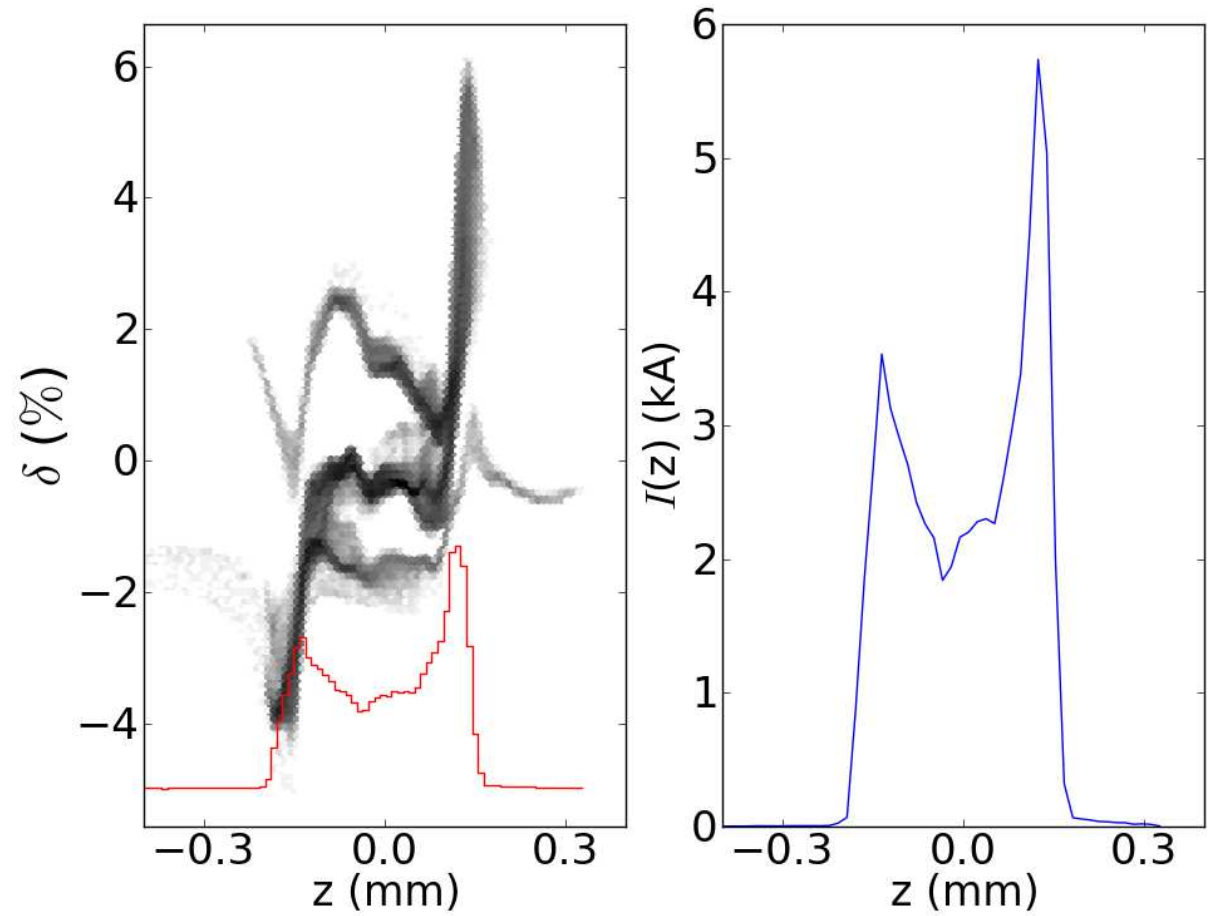

Figure 4.21: LPS and associated longitudinal charge distribution (red trace) in arbitrary units (left) and current profile (right) for a $3.2-\mathrm{nC}$ bunch over-compressed with $\mathcal{C}=5.50 \mathrm{~m}^{-1}$, simulated in IMPACT-Z. 


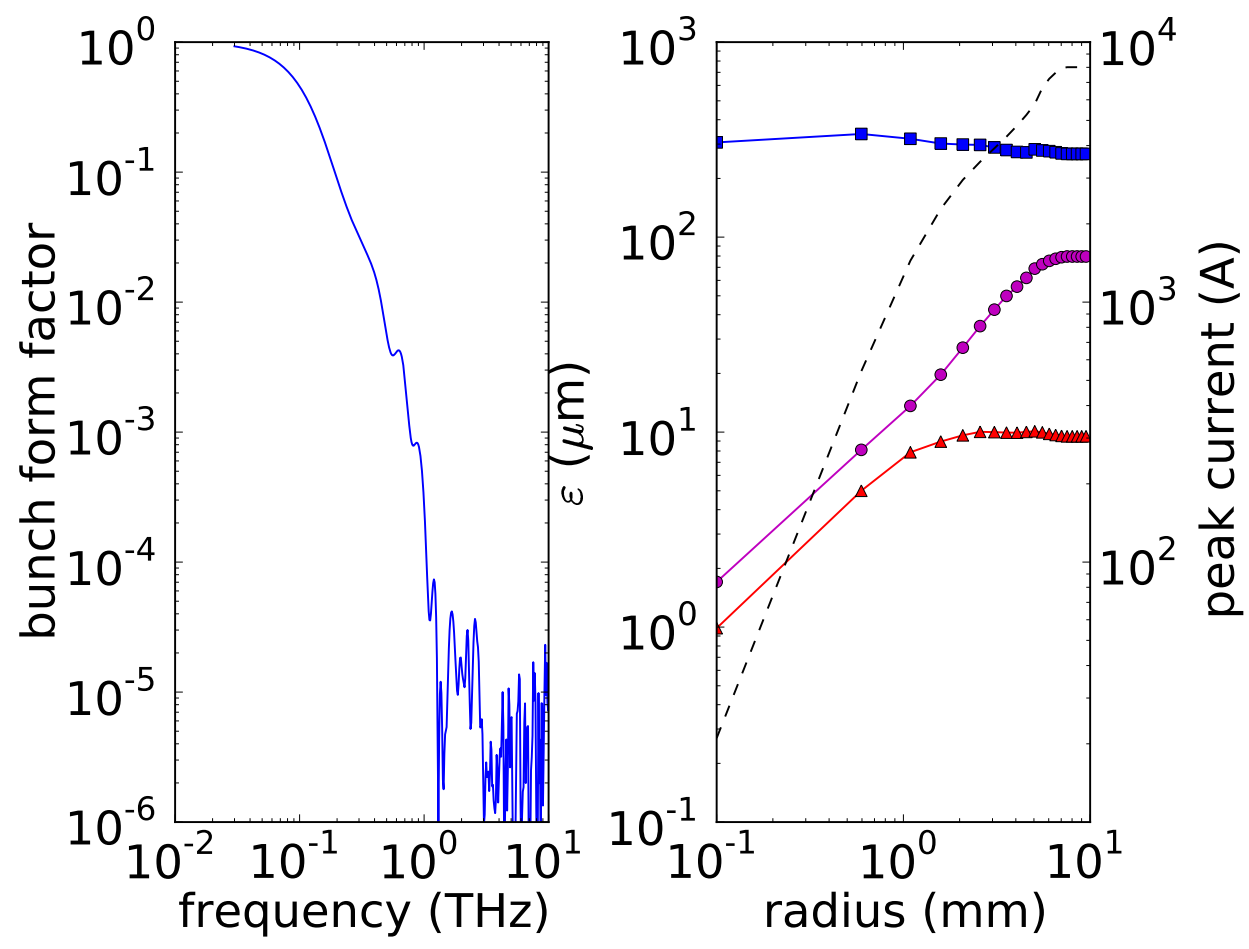

Figure 4.22: Bunch form factor associated to the 3.2-nC fully-compressed electron bunch (left plot) and final horizontal (circles), vertical (triangles), and longitudinal (squares) normalized emittances (left vertical axis) and peak current (dashed lines and dots, right axis) of the bunch within a selected transverse radius (right plot). These simulations were performed near maximum compression with $\mathcal{C}=5.2 \mathrm{~m}^{-1}$ and 3.2-nC. 

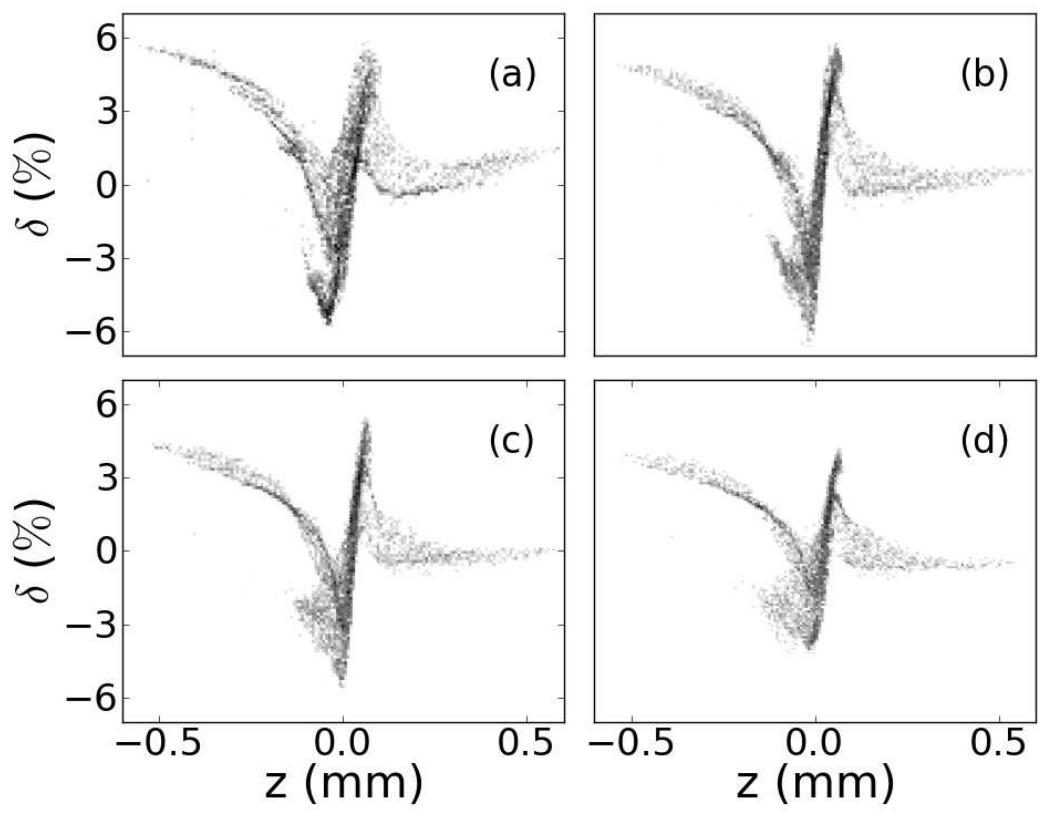

Figure 4.23: Final LPS for initial flat beam aspect ratios of 1 (a), 25 (b), 100 (c), and $400(\mathrm{~d})$. 


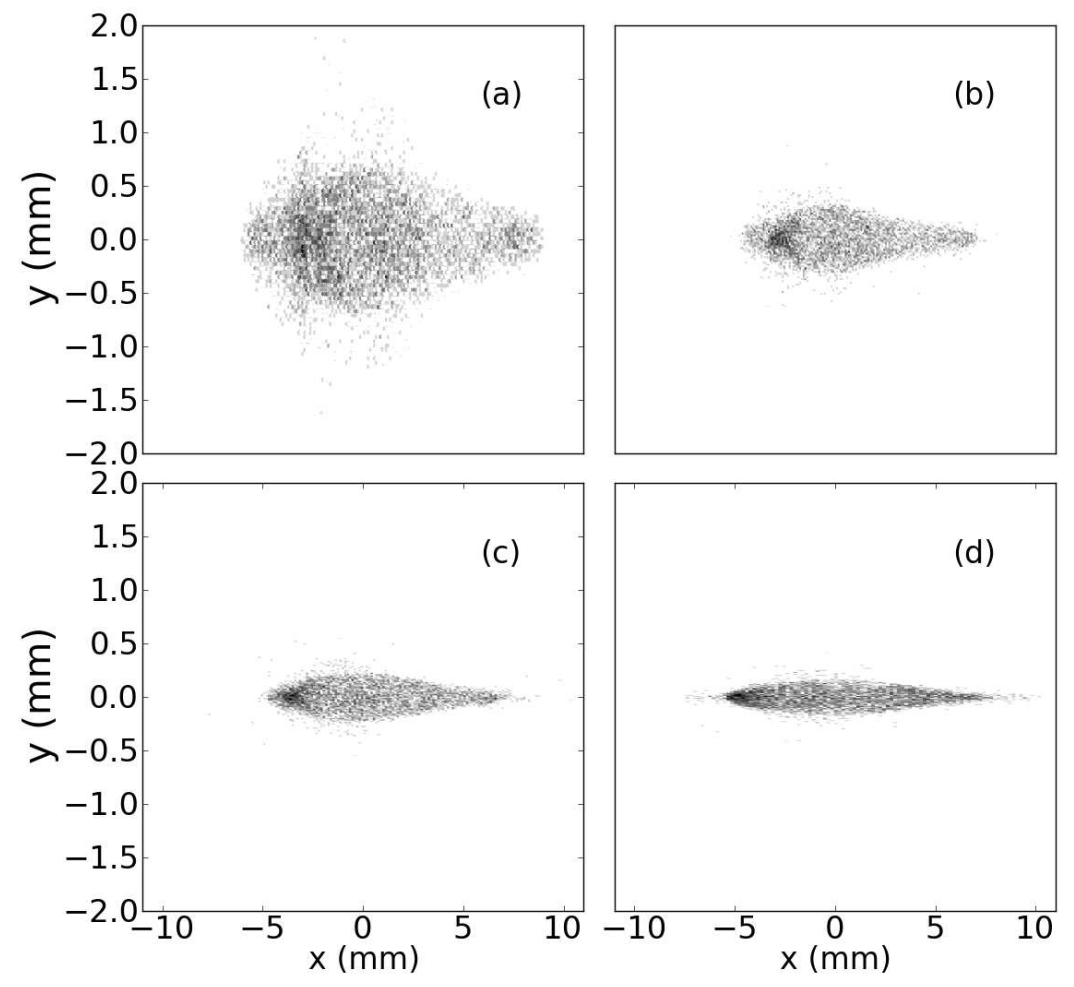

Figure 4.24: Final $x$-y profiles for initial aspect ratios of 1 (a), 25 (b), 100 (c), and 400 (d). 


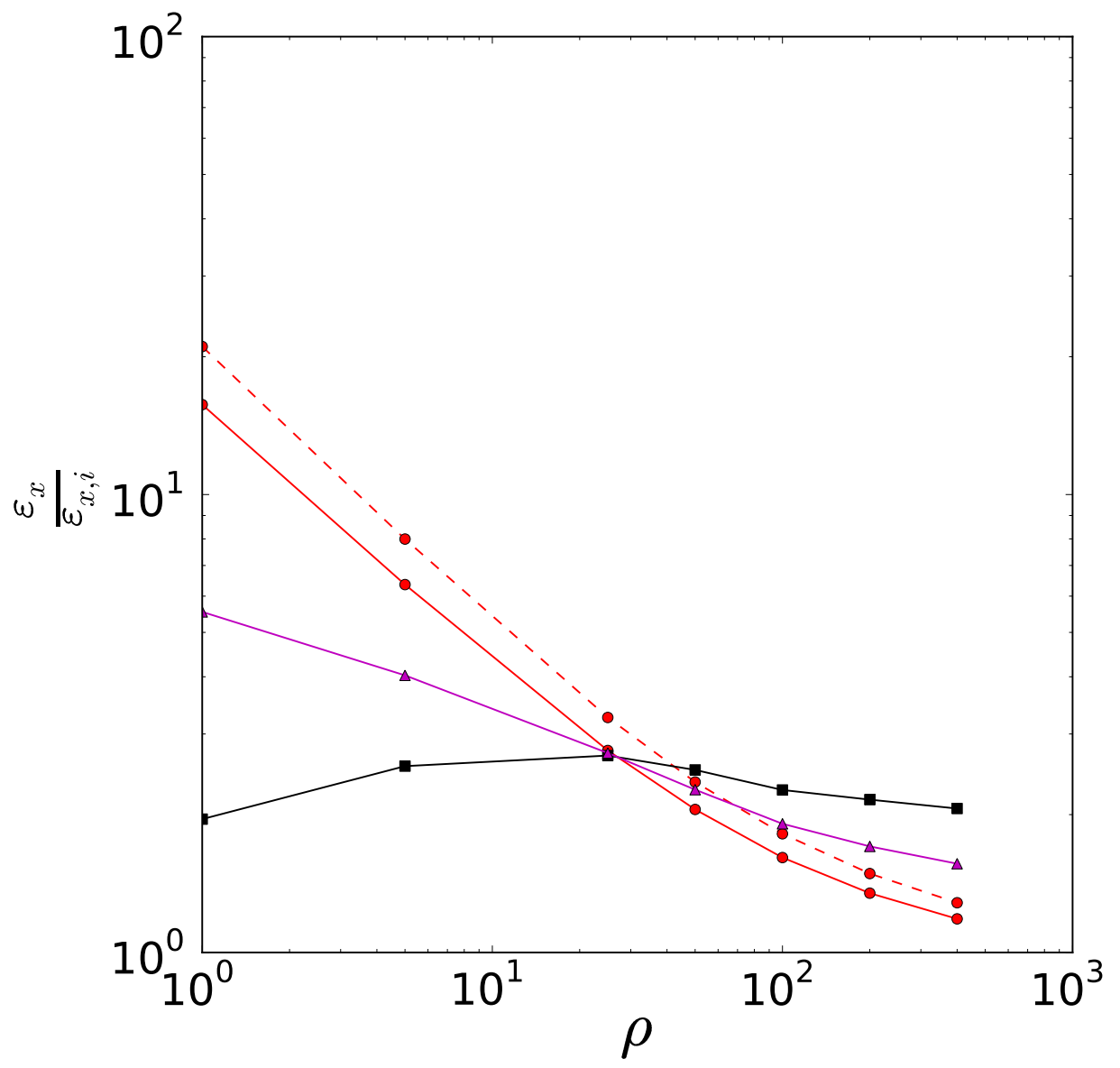

Figure 4.25: Bending plane transverse emittance $\frac{\varepsilon_{x}}{\varepsilon_{x, i}}$ growth in BC1 (circles) simulated with CSRTRACK (dashed line) and IMPACT-Z (solid lines) as functions of the initial emittance ratio $\rho \equiv \frac{\varepsilon_{x, i}}{\varepsilon_{y, i}}$. Corresponding IMPACT-Z results for the vertical emittance $\left(\frac{\varepsilon_{y}}{\varepsilon_{y, i}}\right.$, squares $)$, and four-dimensional transverse emittance $\left(\frac{\varepsilon_{4}}{\varepsilon_{4, i}}\right.$, triangles $)$. 

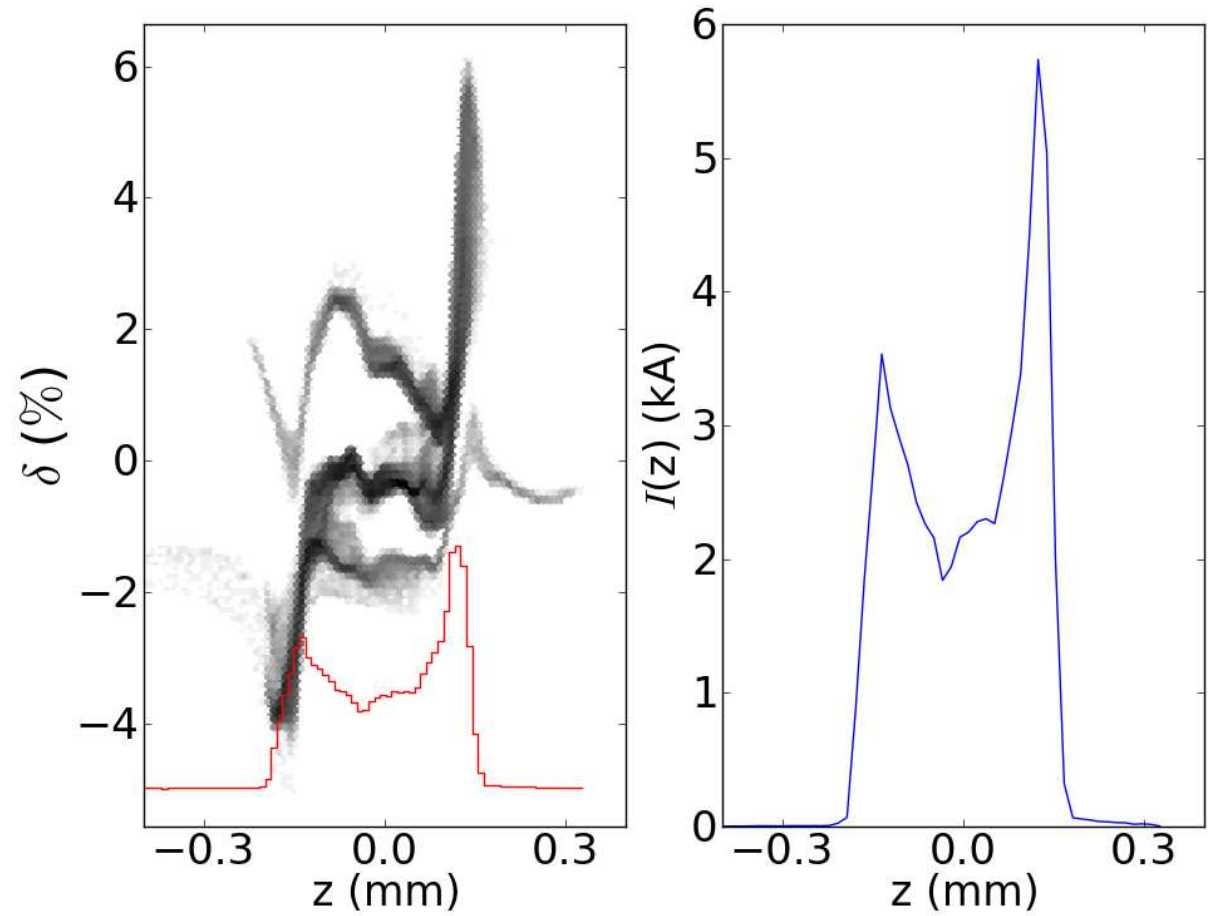

Figure 4.26: LPS distribution (gray colormap in left plot) and current projection (lower trace and right plot) associated to over-compressed bunches with an incoming LPS chirp of $\mathcal{C}=5.5 \mathrm{~m}^{-1}$. The ordinates $z>0$ correspond to the head of the bunch. 


\section{CHAPTER 5}

\section{LONGITUDINAL PHASE SPACE MEASUREMENT}

In the first operating phase, this $\mathrm{BC}$ will be the only one and will most probably be operated to maximally compress the bunch resulting in peak currents in excess of $10 \mathrm{kA}$ as predicted by simulations [51]. Eventually this low-energy BC will be part of the multi-stage compression scheme. To investigate the performance of this low-energy BC, the experiment is set to include LPS diagnostics comprised of a transverse-deflecting cavity (TDC) followed by a vertical spectrometer. In this chapter, we discuss the design, optimization, and anticipated performances of the diagnostics with the help of single-particle-dynamics simulations carried out with ELEGANT. The capabilities and limitations of the diagnostics are also illustrated for realistic LPS distribution simulated with ASTRA.

\subsection{Beamline Design}

The single-shot LPS is comprised of a TDC operated at zero-crossing that shears the beam in the horizontal plane, followed by a dipole magnet that energy disperses the beam in the vertical plane. The two processes combine to map the longitudinal phase space (LPS) in to the $(x, y)$ configuration space that can then be directly 
measured using a standard density-monitor screen [31]. Therefore at the observation location we have

$$
\begin{aligned}
x & \simeq \mathcal{S}_{x} z_{0}+\mathcal{O}\left(x_{0}, x_{0}^{\prime}\right) \\
y & \simeq \eta_{y} \delta_{0}+\mathcal{O}\left(y_{0}, y_{0}^{\prime}\right)
\end{aligned}
$$

where $\left(z_{0}, \delta_{0}\right)$ are the coordinates in the LPS $(\delta$ is the relative fractional momentum offset), and $\eta_{y}$ and $\mathcal{S}_{x}$ are the dispersion and shearing factor computed at the screen location. The latter equation ignores remaining coupling as discussed below, and the subscript 0 indicates quantities upstream of the TDC. In the thin-lens approximation $\mathcal{S}_{x}=\kappa L$ where $L$ is the distance between the TDC and observation screen and the normalized deflecting strength $\kappa$ are respectively the electronic charge, the speed of light, the beam momentum, the integrated deflecting voltage, and the wavelength of the deflecting mode.

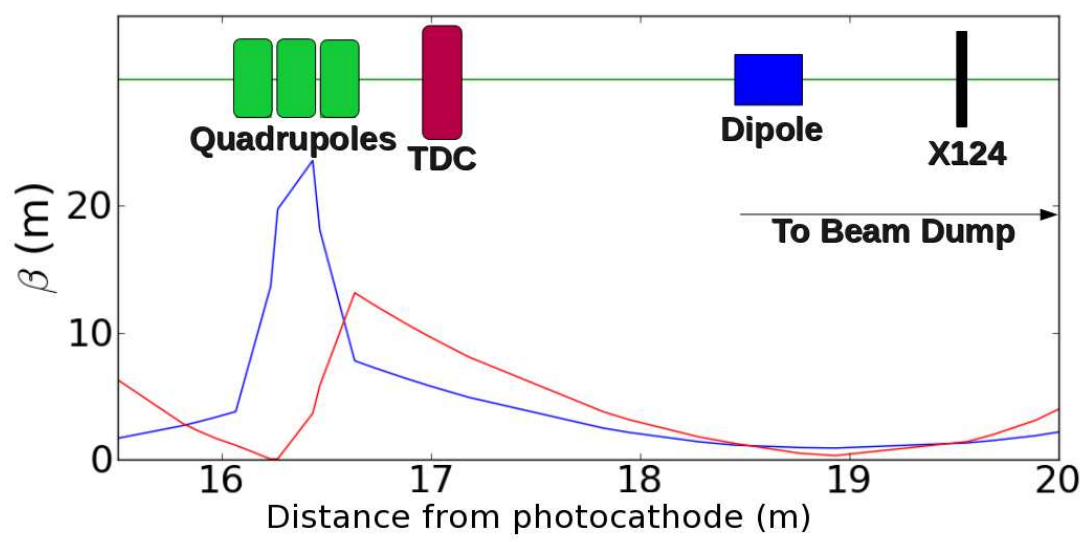

Figure 5.1: Overview of the LPS diagnostic section with associated horizontal (blue) and vertical (red) betatron function. The dispersion at X124 is $\eta_{y}=0.43 \mathrm{~m}$.

Due to space constraints, the observation screen (X124 in Figure 5.1) is located at a distance $L=2.5 \mathrm{~m}$ downstream of the TDC. One of the requirements is the 
ability to quickly diagnose the beam without significantly disrupting the photoinjector magnetic lattice. Several options were considered (including the optimization of the spectrometer dipole entrance/exit face angle) and the most flexible solution that could accommodate the large variation in beam parameters arising from the wide range of operating charges (typically $Q \in[0.02,3.2] \mathrm{nC}$ ) was to use a triplet of quadrupole magnets located upstream of the TDC. The diagnostics beamline is diagrammed in Figure 5.1, beginning downstream of the exit of the low-energy bunch compressor. Given the incoming Courant-Snyder (C-S) parameters, the quadrupole triplet is set to minimize the betatron functions in both planes at X124's location. The TDC is a 5-cell 3.9-GHz cavity [?] similar to the one used at the Fermilab's A0 photoinjector [?]. In its initial design it will be a Nitrogen-cooled normal-conducting cavity and will eventually be upgraded to a superconducting cavity.

Several limiting factors prevent an exact mapping of the LPS into the $(x, y)$ space. First, as noted in Eq. 5.1 and 5.2, contributions from the initial transverse emittances [the $\varepsilon_{x, 0}$ in $\mathcal{O}\left(x_{0}, x_{0}^{\prime}\right)$ and $\varepsilon_{y, 0}$ in $\mathcal{O}\left(y_{0}, y_{0}^{\prime}\right)$ terms] call for small betatron functions at X124. Second, the TDC actually imparts a correlation within the LPS due to its non-vanishing $R_{65} \equiv\langle\delta \mid z\rangle$ [52]. Finally, a significant increase in energy spread can arise from the transverse dependence of the axial electric field $\left(E_{z} \propto x\right)$ in the TDC. These limiting effects impose conditions on the beam parameters that can be casted as

$$
\begin{array}{r}
\eta_{y}^{2} \sigma_{\delta, 0}^{2} \gg \beta_{y} \tilde{\varepsilon}_{y, 0}, \text { and } \\
\mathcal{S}_{x} \sigma_{z, 0}^{2} \gg \beta_{x} \tilde{\varepsilon}_{x, 0},
\end{array}
$$

where the $\beta$ s and $\sigma \mathrm{s}$ are respectively the betatron function at X124 and the initial root-mean square (RMS) beam parameters and the $\tilde{\varepsilon}$ s are the geometric emittances. 
For the simulations presented in this paper, a strength of $\kappa=2 \mathrm{~m}^{-1}$ is assumed as it corresponds to the maximum achievable value given the available RF power and past experience. Given the simulated macroparticle distribution at X124, the coordinates of the macroparticles in the reconstructed LPS $\left(z_{r}, \delta_{r}\right)$ are found from the transformations

$$
\begin{aligned}
z_{r} & =\frac{x}{\kappa L}, \text { and } \\
\delta_{r} & =\frac{y}{\eta_{y}}-R_{65} z .
\end{aligned}
$$

To illustrate the effect of emittance, we use a grid-like LPS, as shown in Figure 5.2, which gives a clear insight as to the underlying mechanism and complications that arise from the transverse emittance. In the case of an ideal zero-emittance beam, the LPS maps perfectly on to the $x-y$ plane, with a "tilt" due to the nonvanishing $R_{65}$ component, which can be corrected when scaling back down to recover the initial LPS.

When a transverse emittance is added, it contributes to the spot size, as demonstrated in Figure 5.2. This effect increases the recovered bunch length and energy spread, and its uncorrelated nature with respect to $z$ increases error in the longitudinal emittance measurement.

\subsection{Transverse Collimation}

In addition to the emittance itself, transversely-dependent energy gains from the TDC map the incoming $x-x^{\prime}$ distribution to the $y$ plane. Collimation can be 

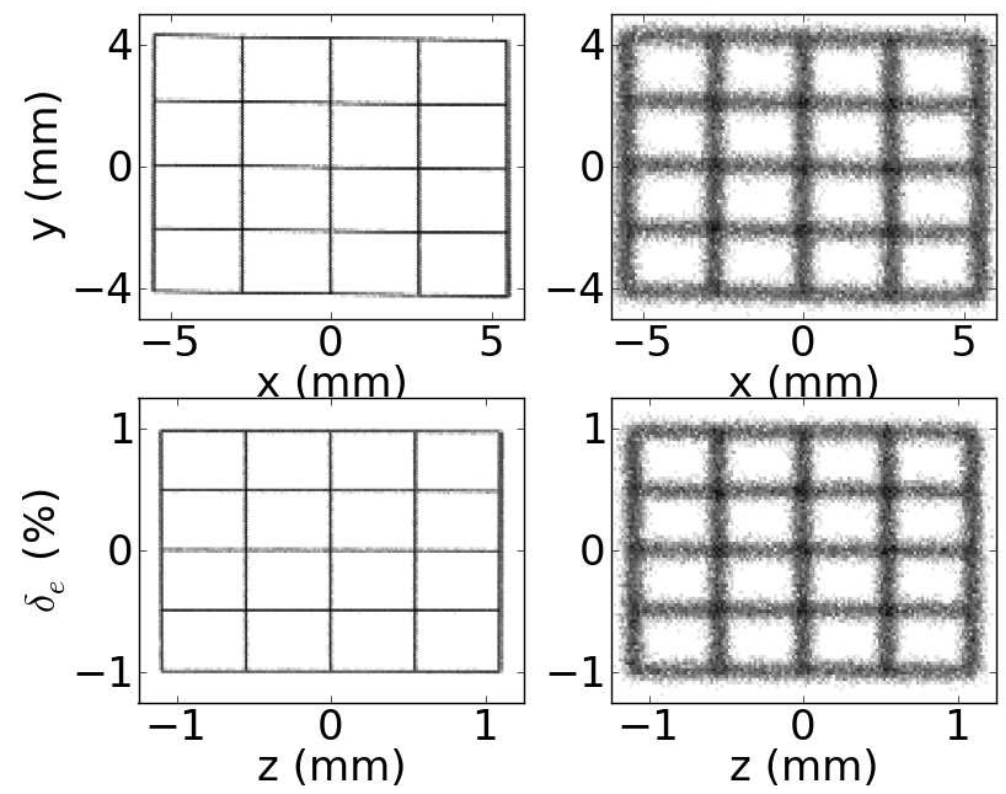

Figure 5.2: The beam profile at the screen (top row) and reconstructed LPS distributions (bottom row) for transverse normalized emittances $\varepsilon_{x}=\varepsilon_{x}=0.0075 \mu \mathrm{m}$ (left column) and $0.75 \mu \mathrm{m}$ (right column), showing the blurring effect that results from the transverse distribution that increases with the emittance, the tilt that is a result of the $R_{65}$ term of the TDC transfer matrix, and the reconstruction process.

used to reduce these transverse contributions to the spot imaged at the screen. In Figure 5.3, we us a realistic bunch distribution obtained from AsTRA simulations of the ASTA photoinjector. For these simulations the charge per bunch is $Q=3.2$ $\mathrm{nC}$ and the bunch is compressed using a magnetic bunch compressor. The observed LPS distortion arises from the quadratic correlation imposed by the RF wave form during acceleration in the photoinjector; see Reference [34]. The distribution is tracked using ELEGANT throughout the LPS-diagnostics beamline. A screen with a $100 \mu \mathrm{m}$ square hole placed at the entrance of the TDC significantly improves the accuracy of the recovered image.

As the aperture size increases, the reconstructed slice energy spectrum smears out, resulting in an over-estimate of the RMS fractional energy spread. This ef- 


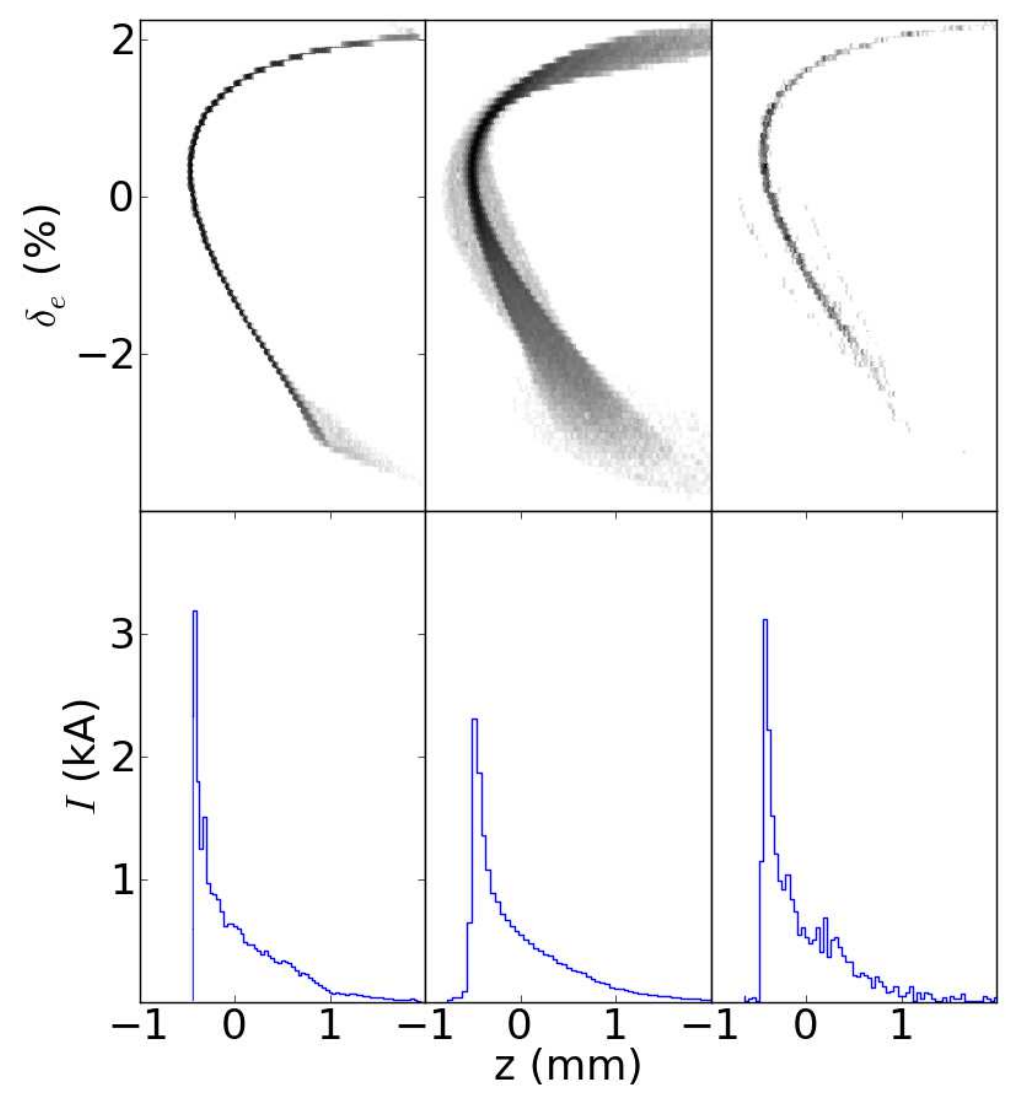

Figure 5.3: LPS distributions (top row) generated using AsTRA after BC1 (left column), reconstructed from the screen without transverse collimation (middle column), and reconstructed with collimation using a 100- $\mu \mathrm{m}$ square aperture (right column), with each LPS's current profile beneath it in the bottom row.

fect is illustrated in Figure 5.4 where energy histograms of macroparticles in the center-most $180-\mu \mathrm{m}$ longitudinal slice (the one with the highest current) of the reconstructed LPS distribution are compared for different collimator sizes with the original LPS. For these simulation an idealized Gaussian bunch with following parameters was considered: RMS bunch length $\sigma_{z}=2.56 \mathrm{~mm}$, RMS slice energy spread $\sigma_{\delta_{e}}=0.04 \%$, energy $\operatorname{chirp} \mathcal{C}=2 \mathrm{~m}^{-1}$, and a normalized transverse emittances of $\varepsilon_{x, y}=5$ $\mu \mathrm{m}$. 


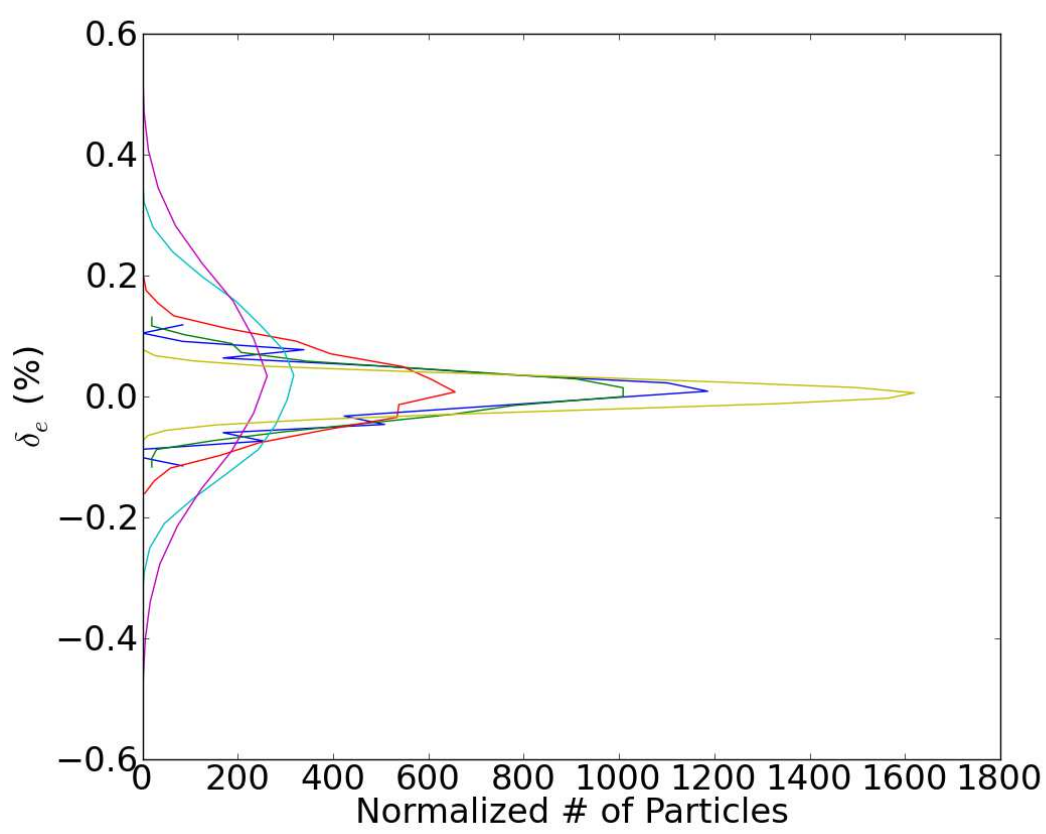

Figure 5.4: Area-normalized fractional-energy-spread histograms of macroparticles located in the center-most $180-\mu \mathrm{m}$ longitudinal slice of the reconstructed LPS for a chirped Gaussian beam, for square collimators with sizes of 50 (blue), 140 (green), 387 (red), 1000 (cyan), and 3000 (magenta) $\mu \mathrm{m}$, and the pre-TDC LPS (yellow). The histograms are normalized to account for the number of particles that are lost to the collimation.

\subsection{Resolution}

In order to quantify the resolution of the proposed beamline we consider a pointlike initial LPS distribution $\Phi\left(z_{0}, \delta_{0}\right)=N \delta\left(\delta_{0}\right) \delta\left(z_{0}\right)$ where the number of macroparticles $N \in\left[2.5 \times 10^{4}, 2.5 \times 10^{5}\right]$, the upper limit being used only for the case of a square collimator due to the large percentage of particles that are lost. The macroparticles are distributed according to a Gaussian distribution in the transverse trace spaces with appropriate initial C-S parameters (which match the ones displayed in Figure 5.1) and variable transverse emittances. The final RMS sizes of 
the recovered LPS using the data simulated at X124 provides the resolution of the LPS diagnostics. After proper calibration, the RMS size in $x$ and $y$ respectively provide the resolution in energy and longitudinal coordinate (or time). In these studies we also investigate the effect of collimation using slits or apertures, of types similar to those presented in the previous section. Figure 5.5 summarizes the inferred time (left plot) and energy (right plot) resolutions for the different configurations. As expected, collimating the initial distribution in the $x$ direction can considerably improve the overall resolution and is especially beneficial in improving the energy resolution.
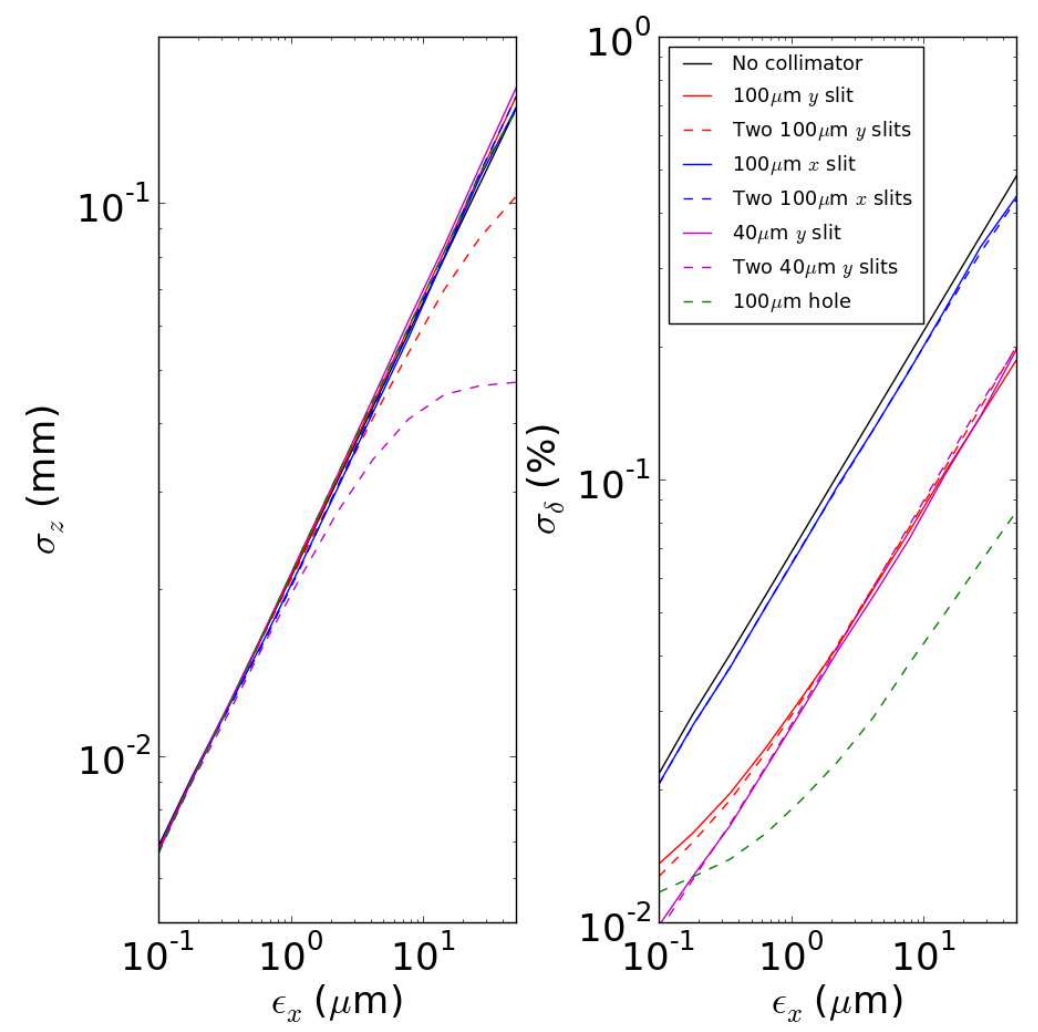

Figure 5.5: Longitudinal-coordinate (left) and fractional energy spread (right) resolutions as a function of initial normalized emittances, $\varepsilon_{x}=\varepsilon_{y}$, for different collimation scenarios (see legend). 


\subsection{Testing at HBESL}

A single-shot LPS measurement system with a similar configuration was tested at A0/HBESL. It is of the same basic configuration as that shown earlier in Figure 5.1, but with different properties. In Table 5.1, beam and configuration parameters are listed to summarize the difference between the two system, with reference to the parameters in Figure 5.6.

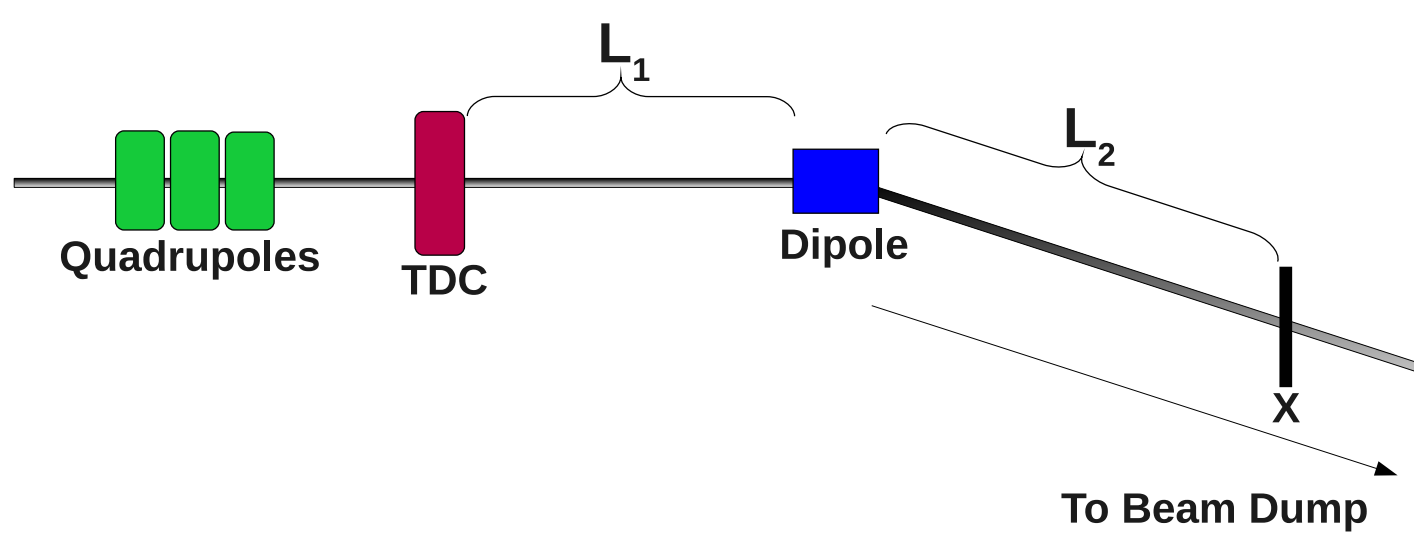

Figure 5.6: Generic layout of the LPS diagnostic system. Comparative values between A0 and ASTA are shown in Table 5.1.

Preparation for a full test of the LPS diagnostic system required several preliminary studies and tunings. These included tuning of the overall beamline, as well as testing the performance of the transverse deflecting cavity. The screen (named X07 and shown in Figure 5.7) is used to find the relationship between length and number of pixels. The screen is tilted $45^{\circ}$ in $y$ (which is the horizontal dimension for the beam), and is one inch in each of the two dimensions. 
Table 5.1: Comparison of bunch and beamline parameters relevant to the LPS measurement system.

\begin{tabular}{cccc}
\hline \hline Property & Value (A0) & Value (ASTA) & Units \\
\hline Beam Energy & 3.5 & 40 & $\mathrm{MeV}$ \\
Bunch Charge & 1.5 & 3.2 & $\mathrm{nC}$ \\
$\mathrm{L}_{1}$ & 1.38 & 2.43 & $\mathrm{~m}$ \\
$\mathrm{~L}_{2}$ & 1.05 & 1.09 & $\mathrm{~m}$ \\
Dispersion at screen & & 0.42 & $\mathrm{~m}$ \\
Bend Angle & 45 & 22.5 & degrees \\
$f_{T} D C$ & 1.3 & 3.9 & $\mathrm{GHz}$ \\
$\lambda_{T} D C$ & 0.2306 & 0.0769 & $\mathrm{~m}$ \\
\hline \hline
\end{tabular}

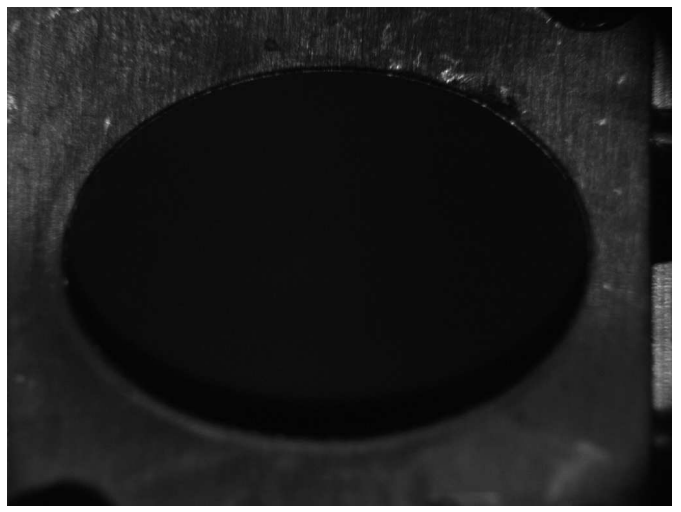

Figure 5.7: Photograph of cross X07 illuminated with a light bulb. The center screen has a diameter of, and is tilted $45^{\circ}$ in $y$.

From the image, the correlations of $0.0307 \mathrm{~mm} /$ pixel in $x$ and $0.0435 \mathrm{~mm} /$ pixel in $y$ were recovered. The horizontal extent $y$ is greater than that in $x$ by roughly a factor of $\frac{1}{\cos (45)}$ due to the tilt of the screen. With the calibration known, we could then proceed onto analyzing the kick strength of the TDC scan.

At the time of the phase scan study, the beam jitter was still significant. As can be seen in Figure 5.8, there is a fairly substantial bunch-to-bunch fluctuation. This is partially from the laser and accelerating cavity, as well as possible timing between the TDC and the bunch. We took five data points at each phase and 
removed extreme outliers. Additional error is introduced from the weak signal and its interaction with the fitting algorithm utilized by the scanning software. In some circumstances, the signal is weak relative to the noise, and a Gaussian fit products what is essentially a spurious solution. However, these effects do not seem to have had a negative impact on our ability to discern the kick strength or deflecting voltage.

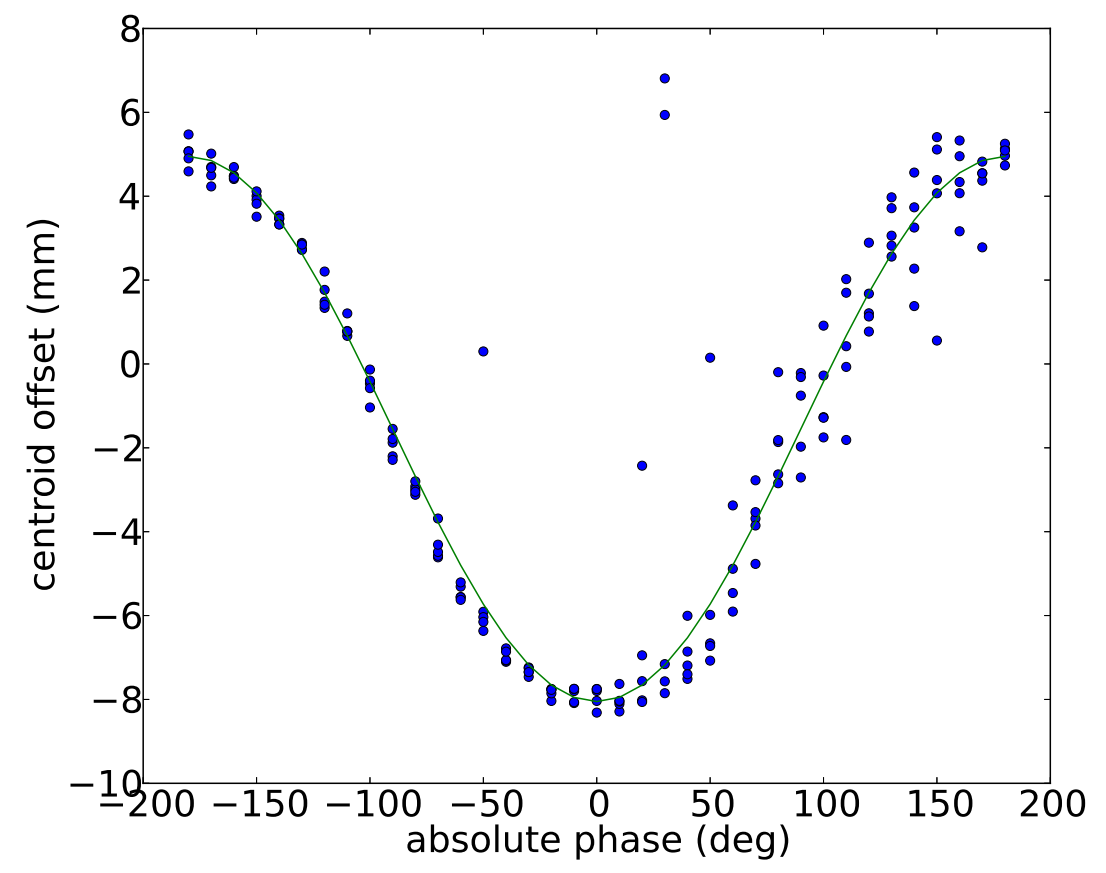

Figure 5.8: Scan of centroid offset versus TDC phase at A0 during original commissioning of the TDC. Several outlier points have been removed. There are a few that are results of erroneous fitting.

With a drift length $L$ between the TDC and X07 of $1.22 \mathrm{~m}$, a beam energy $E$ of 3.5 MeV, a peak-to-peak deflection of $13 \mathrm{~mm}$ (calculated by average of points at 0 and 180 degrees), we can find the deflecting voltage $V_{\perp}$ via the relationship

$$
V_{\perp}=\frac{x_{\max } E}{L}
$$


So, the deflecting voltage was $18.6 \mathrm{keV}$. This corresponds to a kick strength, $\kappa$, of $0.435 \mathrm{~m}^{-1}$.

The implementation of the LPS measurement at ASTA will not be ready for its first-beam implementation, as installation of the $3.9 \mathrm{GHz}$ power supplies are at a lower priority than more fundamental parts of the beamline. However, the spectrometer magnet down to the beam dump is still in place. 


\section{CHAPTER 6}

\section{A DESIGN OF AN IN-LINE TRANSVERSE-TO-LONGITUDINAL EMITTANCE EXCHANGER}

\subsection{Introduction}

Advanced applications of particle accelerators generally rely on phase space manipulations to tailor the phase space distributions to match the requirements of the front-end applications. Recently, there has been an increasing demand for more precise phase space control. In particular, electron bunches with tailored temporal distributions are often desired. For instance, the production of trains of microbunches with a given bunch-to-bunch separation is foreseen to pave the road toward compact light source operating in the super-radiant regime at wavelengths comparable to or larger than the typical microbunch length [53].

In addition, novel accelerator concepts based on beam-driven acceleration mechanisms, e.g., plasma or dielectric wakefield-based acceleration [54, 55, 56, 45], would greatly benefit from linearly ramped or trapezoidal current profiles $[43,57]$ to significantly increase the transformer ratio - the energy gain of the accelerated bunch over the energy loss of the driving bunch [54].

To address these needs, several techniques aimed at tailoring the current profile of electron bunches have been explored. Linearly-ramped current profiles can be produced by imparting nonlinear distortions in the longitudinal phase space 
(LPS) $[58,59,44]$. The generation of a train of microbunches by shaping the temporal profile of the photocathode drive laser in a photo-emission electron source were explored via numerical simulations $[60,61,62]$. An alternative method using an interceptive mask located in a dispersive section, first proposed in Reference [63], was experimentally demonstrated [64].

Recently, techniques relying on the exchange of transverse and longitudinal phase space have emerged. The emittance exchange was first proposed in the context of B-factory [65] as a way to achieve very small $\beta^{*}$ values at the interaction point. The scheme was later explored as a possible alternative for mitigating microbunching instability in bright electron beams [66] or for improving the performance of single-pass FELs [67]. There are several solutions capable of performing this phase space exchange $[68,69,70]$. The simplest solution devised to date consists of a horizontally-deflecting cavity, operating on the $\mathrm{TM}_{110}$ mode, flanked by two identical horizontally-dispersive sections henceforth referred to as "dogleg" [67]; see Figure 6.1 .

An experiment conducted at the Fermilab's A0 photoinjector facility demonstrated the exchange of transverse and longitudinal emittances using the latter "double-dogleg" emittance exchanger (DDEEX) configuration [71] along with its ability to shape the longitudinal distribution [72]. A major disadvantage of the DDEEX beamline stems from the resulting offset of the beam's direction which has significant impact on linac design as an elaborate dispersion correction scheme would be needed to operate the beamline in its non emittance-exchanging configuration.

To circumvent this major limitation, chicane- and arc-like emittance exchangers have been proposed [70] based on earlier work on general emittance-exchange conditions [68]. Geometrically, one possible solution consists of a beamline with its second dogleg flipped compared to the DDEEX configuration; see Figure 6.1. Compared to 

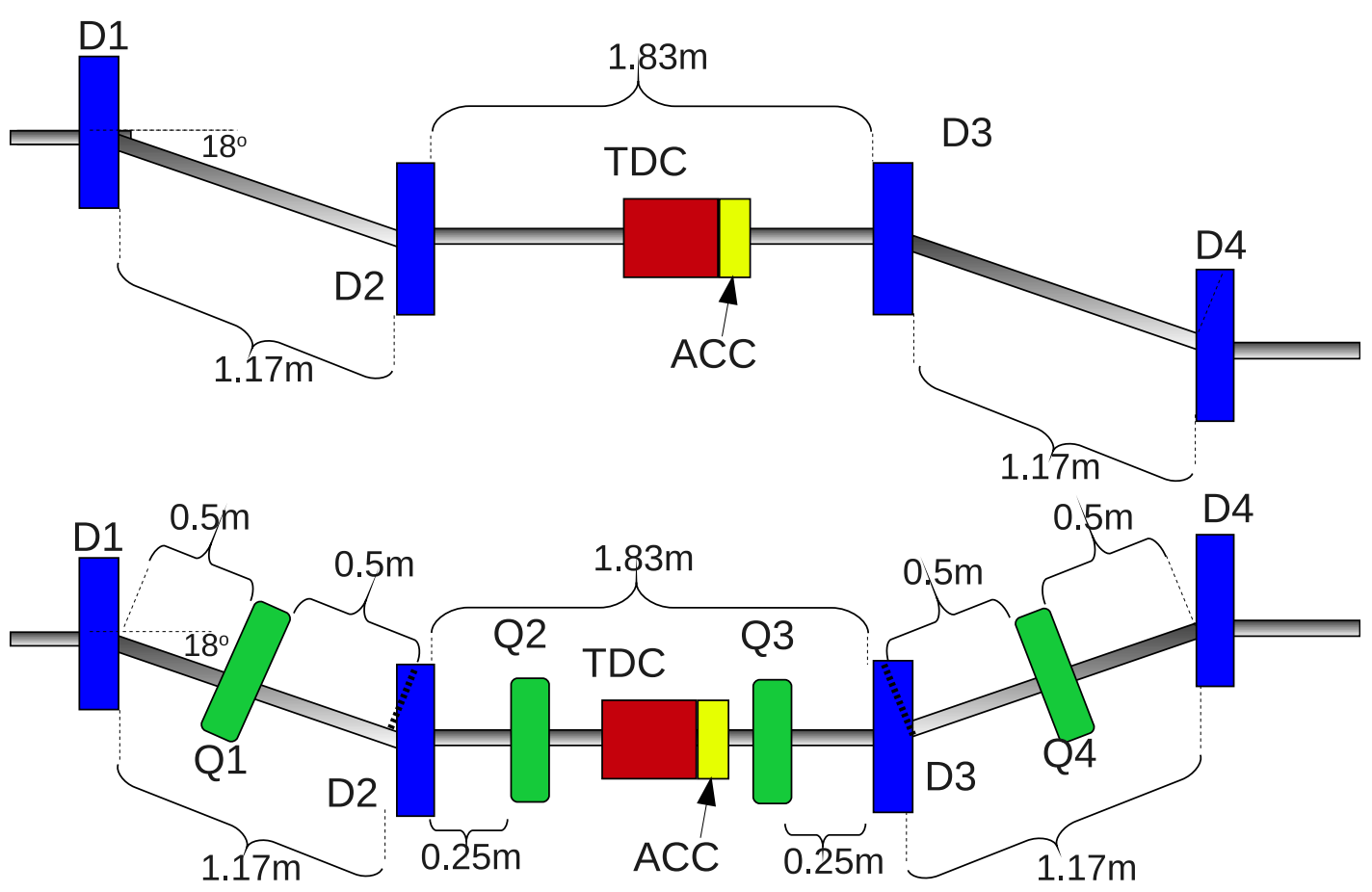

Figure 6.1: The DDEEX (top) and chicane-like (bottom) emittance-exchanger configurations. The two use the same lengths, elements, and bend angles, but the NDEEX reverses the bend angles of the downstream dogleg and includes additional quadrupole magnets. The quadrupole magnets may also be used to increase the dispersion at the TDC location, which relaxes the requirements on the cavity strength. Dipole magnets have a projected length of $0.30 \mathrm{~m}$, and the quadrupole magnets are $0.17 \mathrm{~m}$ long. The TDC is centered between B2 and B3, and an accelerating mode cavity is placed immediately downstream.

the initial design devised in Reference [66], the chicane-like EEX includes quadrupole magnets for dispersion and beam-envelope control and the exchange can be made exact to first order (contrary to the chicane initially devised in Reference [66]).

The main goal of this study is to provide a comprehensive comparison between the DDEEX and chicane-like configurations. We first review the emittance exchange conditions and discuss two possible chicane-like EEX configurations. We then present numerical simulation of the beam dynamics of different EEX beamline including collective effects such as space charge (SC) and coherent synchrotron ra- 
diation (CSR). Our studies focus on attempting an ideal exchange of the horizontal and longitudinal emittances and on producing electron bunch with shaped current profiles.

\subsection{Basics of Phase-space-Exchanging beamlines}

In this Section we summarize the required conditions for phase space exchange and detail the three beamline configurations we investigate. References $[67,73,70]$ give a thorough description of the matrix formulation of EEX beamlines. Here we note some difference with previous work and follow the notation introduced in [73]. We define the six-dimensional trace space coordinates as $\left[x, x^{\prime}, y, y^{\prime}, z, \delta\right]$ where the prime refer to the divergence, e.g., $x^{\prime} \equiv p_{x} / p_{z}$ [where $p_{i}(i \in[x, y, z])$ are the components of the momentum] and $\delta \equiv p /\langle p\rangle-1$ is the fractional momentum spread. We take the EEX beamline to exchange phase space coordinates between the horizontal $\left(x, x^{\prime}\right)$ and longitudinal $(z, \delta)$ phase spaces. Hence for most of the paper we introduce the four-dimensional coordinate $\widetilde{\mathbf{X}} \equiv\left(x, x^{\prime}, z, \delta\right)$ where $\sim$ corresponds to the transposition operator. In this four-dimensional sub-space and under linear dynamics, a particle with initial coordinate vector $\mathbf{X}_{\mathbf{0}}$ is transformed according to

$$
\mathbf{X}_{\mathbf{0}} \stackrel{R}{\longrightarrow} \mathbf{X}=\left(\begin{array}{cccc}
R_{11} & R_{12} & R_{15} & R_{16} \\
R_{21} & R_{22} & R_{25} & R_{26} \\
R_{51} & R_{52} & R_{55} & R_{56} \\
R_{61} & R_{62} & R_{65} & R_{66}
\end{array}\right) \mathbf{X}_{\mathbf{0}}
$$

where $R$ is the usual transport matrix with its elements $R_{i j}$ indexed to conform with the $6 \times 6$ matrix convention. 
An ideal EEX beamline has an anti-diagonal transfer matrix of the form $R=$ $\left(\begin{array}{ll}0 & B \\ C & 0\end{array}\right)$ where $B$ and $C$ are $2 \times 2$ matrices. The anti-diagonal nature of the transfer matrix exchange phase space coordinate between the horizontal and longitudinal degrees of freedom and consequently the horizontal and longitudinal emittances.

Considering a beamline composed of a deflecting cavity flanked by two dispersive sections, the condition for phase space exchanges can be summarized as

$$
\begin{aligned}
\mathbf{D}_{+} & =\left(\begin{array}{cc}
R_{11,+} & R_{12,+} \\
R_{21,+} & R_{22,+}
\end{array}\right) \mathbf{D}_{-}, \text {and } \\
\kappa & =-1 / \eta_{-}
\end{aligned}
$$

where the "+" and "-" signs refer to values associated to respectively the downstream and upstream dispersive sections and $\widetilde{\mathbf{D}} \equiv\left(\eta, \eta^{\prime} \equiv d \eta / d s\right)$ is the dispersion vector. In Eq. 6.2 and 6.3 we took the transfer matrix of the deflecting cavity (with its length $L$ set to zero) as

$$
R_{T D C}(\kappa, L)=\left(\begin{array}{cccc}
1 & L & \kappa \frac{L}{2} & 0 \\
0 & 1 & \kappa & 0 \\
0 & 0 & 1 & 0 \\
\kappa & \kappa \frac{L}{2} & \kappa^{2} \frac{L}{4} & 1
\end{array}\right),
$$

where $\kappa$ is the TDC normalized strength [52] defined as $\kappa \equiv \frac{2 \pi}{\lambda} \frac{e V_{x}}{p c}$, where $e, c$, $p_{0}, V_{x}$ and $\lambda$ are respectively the electron charge, speed of light, beam momentum, integrated deflecting voltage, and the wavelength of the deflecting mode. A cell of 
length $L=\frac{\lambda}{2}$ is typically used such that the fields experienced are zero as the beam enters and exits the cavity.

A full TDC is comprised of multiple cells, each of which has a proportionally smaller kick $\kappa$. This has the effect of reducing the $R_{65}$ from the $\kappa^{2}$ dependence.

Note that in Eq. 6.3, $\eta_{-}$is the dispersion generated by the upstream dispersive section at the TDC location. When thick-lens effects $(L \neq 0)$ are taken into account the non-vanishing $R_{56}$ element in the TDC transfer matrix introduces residual coupling that degrades the phase-space exchange (a complete phase-space exchange is possible pending the beam's incoming longitudinal phase space chirp is properly selected [67]). It was shown that placing an accelerating cavity operating at zero-crossing downstream of the TDC cancels the residual coupling and render the transfer matrix of the EEX beamline exactly block anti-diagonal [74].

It should be emphasized that Eq. 6.3 implies a TDC deflecting voltage amplitude $\left|e V_{x}\right|=\frac{2 \pi \eta_{-}}{\lambda p c}$ which can be substantial for high-energy beams. This requirement can be relaxed by boosting the value of the dispersion $\eta_{-}$at the cavity location at the expense of a larger horizontal beam size at the TDC location. Beamline designs that allow for higher dispersions are explored in this paper.

In addition to swapping the emittances between the transverse and longitudinal degrees of freedom, an EEX beamline can also act as a longitudinal pulse shaper and support the production of bunches with arbitrarily-shaped current profiles. Assuming an ideal anti-diagonal transfer matrix for the EEX beamline, the final LPS coordinates $\left(z_{f}, \delta_{f}\right)$ of a particle are solely functions of the incoming transverse coordinates $\left(x_{0}, x_{0}^{\prime}\right)$ following

$$
\begin{aligned}
z_{f} & =R_{51} x_{0}+R_{52} x_{0}^{\prime}, \\
\delta_{f} & =R_{61} x_{0}+R_{62} x_{0}^{\prime} .
\end{aligned}
$$


Therefore a shaped initial transverse-phase space distribution obtained by, e.g., using an interceptive mask, can be converted into a shaped current profile downstream of the EEX beamline. For EEX beamlines with $R_{52}=0$, any horizontal shaping imparted onto the beam is ideally mapped into the longitudinal profile with a scaling factor $R_{51}$. Finally, it is often desirable to have an uncorrelated final LPS to minimize the final bunch duration or energy spread. The LPS chirp was defined in Section 4.2.

The final LPS chirp, $\mathcal{C}_{z, f}$, can be expressed as function of the initial horizontal phase space (HPS) $\operatorname{chirp} \mathcal{C}_{x, 0} \equiv\left\langle x_{0} x_{0}^{\prime}\right\rangle / \sigma_{x, 0}^{2}$ as

$$
\mathcal{C}_{z, f}=\frac{R_{61}+\mathcal{C}_{x, 0} R_{62}}{R_{51}+\mathcal{C}_{x, 0} R_{52}}
$$

where we have taken the initial HPS to be correlated such that $x_{0}^{\prime}=\mathcal{C}_{x, 0} x_{0}+\widetilde{x_{0}^{\prime}}$

with $\widetilde{x_{0}^{\prime}}$, the uncorrelated transverse divergence, assumed to verify $\widetilde{x_{0}^{\prime}} \ll \sigma_{x^{\prime}, 0}$. Note that the HTS chirp can be related to the initial Courant-Snyder (C-S) parameter via $\mathcal{C}_{x}=-\alpha_{x} / \beta_{x}$. To obtain a final uncorrelated LPS two conditions are possible:

$$
\mathcal{C}_{x, 0}=-\frac{R_{62}}{R_{61}}, \text { and } \mathcal{C}_{x, 0}=-\frac{R_{52}}{R_{51}}
$$

The latter equations respectively correspond to the case of a minimum final bunch length and minimum final fractional momentum spread. These equations provide some guidance on the required HPS C-S parameters.

Furthermore, we can use additional quadrupole magnets placed upstream of the first dogleg to control the overall $R_{51}, R_{52}, R_{61}$, and $R_{62}$ of the new system to shape the final longitudinal phase space. Likewise, quadrupole magnets placed after the 
emittance exchanger can control the overall $R_{15}, R_{16}, R_{25}$, and $R_{26}$ of the system to shape the final HPS.

\subsection{Simulation Methods}

\subsubsection{Overview}

To carry a comparative beam-dynamics study of the designs detailed in Section 6.4 we use the computer programs ELEGANT [35], IMPACT-Z [75] and, for a reduced set of cases, CSRTRACK [76].

ELEGANT was used to perform single-particle-dynamics simulations. The latticefitting features of ELEGANT were extensively used to seek beamlines with transfer matrices that verify Eq. 6.2. Besides lattice design (which were done to first order), ELEGANT also enabled us to perform aberration studies. We explored second-order effects via particle tracking. For all cases, our studies were limited to the case of initial Gaussian distributions with parameters specified in Table 6.1. The first (case 1 ) is a smaller test set, while the second (case 2) based on partially compressed beam from study in Reference [77].

ELEGANT includes several models for the TDC: it can be modeled using a userprovided transfer matrix, or it can be simulated with the beamline element RFDF. The three models were implemented; the transfer matrix models uses Eq. 6.4 with $R_{T D C}(\kappa, L)$ and $R_{T D C}(\kappa, L=0)$ for respectively the thick- and thin-lens models. A comparison between the various models appears in Appendix B.

Simulations using the program IMPACT-Z were performed using the lattice optimized in ELEGANT to investigate their performances when collective effects are 
Table 6.1: Two sets of initial beam parameters.

\begin{tabular}{ccccc}
\hline Parameter & symbol & $\begin{array}{c}\text { Value } \\
\text { (case 1) }\end{array}$ & $\begin{array}{c}\text { Value } \\
\text { (case 2) }\end{array}$ & Units \\
\hline \hline hor. emittance & $\varepsilon_{x, 0}$ & 1.0 & 10.0 & $\mu \mathrm{m}$ \\
vert. emittance & $\varepsilon_{y, 0}$ & 1.0 & 10.0 & $\mu \mathrm{m}$ \\
long. emittance & $\varepsilon_{z, 0}$ & 10.0 & 39.1 & $\mu \mathrm{m}$ \\
bunch length & $\sigma_{z, 0}$ & 0.4 & 0.8 & $\mathrm{~mm}$ \\
LPS chirp & $\mathcal{C}_{z, 0}$ & 0.0 & 0.0 & $\mathrm{~m}^{-1}$ \\
frac. mom. spread & $\sigma_{\delta, 0}$ & $2.5 \times 10^{-4}$ & $5.0 \times 10^{-4}$ & - \\
\hline
\end{tabular}

taken into account. The capabilities of both ELEGANT and IMPACT-Z are discussed in Appendix C.

Given that ELEGANT has only simply and highly restrictive models for collective effects, we use the tracking code IMPACT-Z for advanced studies, which utilizes a kick-step particle-in-cell method to simulate space charge effects, in addition to an implementation of the 1D CSR model [17]. After ELEGANT is used to determine the strength of the quadrupole magnets, we use a script to convert the design and quadrupole magnet strengths into an IMPACT-Z input file. For the TDC and accelerating cavity, we use field maps generated with HFSS, a high-frequency structure simulator from Ansys.

\subsection{Overview of EEX Designs}

In this Section we summarize the properties of the four beamlines considered through out this paper. Each of the designs must generally satisfy three different requirements, one for each of the three parts described in Eqs. 6.2 and 6.3. For section $M_{-}$, we must control of the upstream dispersive section's $x$ dispersion, $\eta_{-}$, 
and its derivative, $\eta_{-}{ }^{\prime}$. For section $M_{T D C}$, we must control of the strength of the TDC as $\kappa=-\frac{1}{\eta_{-}}$, operated at zero-crossing. For section $M_{+}$, the dispersive section's transfer matrix must be finely controlled. We explore two geometries that satisfy this condition, and variations of one.

\subsubsection{Conventional double-dogleg emittance-exchange (DDEEX) beamline}

The most basic design is the Double Dogleg EEX (DDEEX), which was initially derived in Reference [67]. This design was experimentally demonstrated at A0. The dispersion $\eta$ downstream of each dogleg is $0.5 \mathrm{~m}$, and the TDC is flanked between the doglegs. An accelerating-mode cavity is placed immediately downstream of the TDC to correct for the $R_{65}$ term induced by the TDC. The dipole magnets are rectangular, and the relevant lengths and distances are reported in Figure 6.1 and Table. 6.2.

\subsubsection{Chicane-like, nominal dispersion, emittance-exchange (NDEEX) beamline}

A similar design is the Nominal Dispersion EEX (NDEEX), in which the bend angles and geometry of each dogleg are the same, except the bend angles of one of them are reversed such that it forms a chicane rather than a pair of symmetric doglegs. Such a configuration has imperfect emittance exchange with residual terms in the diagonal blocks of the transfer matrix, and was the first EEX configu- 
ration proposed [8]. For our design, we use four quadrupole magnets placed inside the chicane between each of existing elements. These tune the dispersion and its derivative to satisfy Eq. 6.2. The quadrupole magnets are used to control the sign of the dispersion the upstream dogleg, while the quadrupole magnets of the downstream dogleg are used to meet the new requirements on the transfer matrix from the adjusted Equation 6.2. For the simplest case, we choose the new dispersion of the upstream dogleg, $D_{-}$, to have the same magnitude as that of the DDEEX, i.e., $\eta_{-}=-0.5$

\subsubsection{Chicane-like, boosted dispersion, emittance-exchange (BDEEX) beamline}

The basic configurations are shown in Figure 6.1, while beamline parameters are presented in Table 6.2. Drifts lengths, bend angles, and the overall scale are selected so that it may fit into multiple locations of the ASTA facility.

Table 6.2: Beamline parameters of the EEX configurations in Figure 6.1

\begin{tabular}{ccc}
\hline Parameter & Value & Units \\
\hline \hline Dipole Magnet Length & 0.30 & $\mathrm{~m}$ \\
Dipole Magnet Bend Angle & 18 & degrees \\
Beam Energy & 50 & $\mathrm{MeV}$ \\
$\left|\eta_{-}\right|$ & {$[0.5,1.0,1.5,2.0,2.5,3.0]$} & $\mathrm{m}$ \\
$\left|\eta_{-}^{\prime}\right|$ & 0.0 & $\mathrm{~m}$ \\
$|\kappa|$ & {$[2.0,1.1,0.66,0.5,0.4,0.33]$} & $\mathrm{m}^{-1}$ \\
\hline
\end{tabular}

The EEX configurations represent the basic structure of the DDEEX, NDEEX, and BDEEX configurations that we use for the simulation comparisons in the following section. 
The boosted-dispersion emittance exchanger utilizes the same basic geometry as the NDEEX design, with the key difference being that the strengths of the quadrupole magnets, Q1, Q2, Q3, and Q4, are adjusted to satisfy Eqs. 6.2 and 6.3 for different values of $\eta_{-}$. The TDC strength $\kappa$ is reduced accordingly. As $\eta_{-}$is increased, it becomes more difficult to find quadrupole magnet settings that still satisfy Eqs. 6.2 and 6.3. As $\eta_{-}$increases, it becomes more difficult to adjust $R_{56}$

\subsection{Note on TDC models}

We use several different models for the TDC, which differ in their treatments of the thick-cavity effects as well as the inclusion of higher-order effects. The simplest model is a simple, single-cell deflecting cavity. It has no term associated with its length, and models the deflecting as an instantaneous kick of strength $2.0 \mathrm{~m}^{-} 1=\frac{1}{\eta_{-}}$.. There is no $R_{65}$ term associated with the thin cavity model.

$$
M_{\text {Thin }}=\left(\begin{array}{cccc}
1.0 & 0.0 & 0.0 & 0.0 \\
0.0 & 1.0 & 2.0 & 0.0 \\
0.0 & 0.0 & 1.0 & 0.0 \\
-2.0 & -0.0 & 0.0 & 1.0
\end{array}\right)
$$

Next, the thick-cavity model, which contains finite terms associated with its length, and an $R_{65}$ term. The $R_{12}$ term is the length of the cavity used. 


$$
M_{\text {Thick }}=\left(\begin{array}{cccc}
1.000 & 0.192 & 0.192 & 0.0 \\
0.0 & 1.0 & 2.0 & 0.0 \\
0.0 & 0.0 & 1.00 & 0.0 \\
-2.0 & -0.192 & -0.192 & 1.0
\end{array}\right)
$$

ELEGANT has a deflecting cavity model implemented called RFDF (RF Deflecting cavity)

$$
M_{R F D F}=\left(\begin{array}{cccc}
1.00 & 0.192 & 0.192 & 0.0 \\
0.02 & 1.0 & 2.0 & 0.0 \\
0.0 & 0.0 & 1.00 & 0.0 \\
-2.0 & 0.192 & -0.128 & 1.0
\end{array}\right)
$$

The $R_{65}$ of the RFDF is based on the limit in which the TDC is comprised of multiple cells, as opposed to the thick-cavity matrix of Eq. 6.10 which is that of a single cell cavity. The RFDF also includes second-order effects. To correct for the $R_{65}$ leads to non-zero terms in Equation 6.1 diagonal blocks, so we place an accelerating mode cavity using ELEGANT's RFCA model immediately following the RFDF. The $R_{12}$ and $R_{15}$ terms are increased relative to $M_{R F D F}$ due to the additional length corresponding the accelerating mode cavity. 


$$
M_{R F D F+R F C A}=\left(\begin{array}{cccc}
1.000 & 0.309 & 0.426 & -0.182 \\
0.0 & 1.0 & 2.0 & 0.0 \\
0.0 & 0.0 & 1.00 & 0.0 \\
-2.0 & -0.192 & 0.0 & 1.00
\end{array}\right)
$$

We use a field map for IMPACT-Z of a 5-cell cavity [?], which is generated with HFSS. As in ELEGANT, an accelerating mode cavity is included downstream of the TDC, and is again implemented with a field map.

\subsection{First-Order Comparison of different EEX designs}

We inspect the transfer matrices of both the DDEEX and NDEEX emittance exchangers, and their alternatives without the accelerating mode cavity. The transfer matrix for the two designs, as well as their implementations for which an accelerating mode cavity is excluded, are

$$
M_{D D E E X} \times 10^{3}=\left(\begin{array}{cccc}
0.055 & 0.007 & -5885 & 349 \\
0.016 & -0.007 & -2006 & 289 \\
-289 & -335 & 0.014 & 0.0 \\
2006 & 5780 & -0.011 & 0.0
\end{array}\right) \text {, }
$$




$$
M_{D D E E X n o A c c} \times 10^{3}=\left(\begin{array}{cccc}
3.53 & 52.0 & -5800 & 335 \\
0.02 & 0.005 & -2006 & 0.289 \\
-289 & -330 & 0.019 & -2.67 \\
2006 & 5720 & -128 & 18.6
\end{array}\right)
$$

$$
M_{N D E E X} \times 10^{3}=\left(\begin{array}{cccc}
1.36 & 0.021 & 5870 & 780 \\
0.010 & 0.335 & 2012 & 98 \\
-1380 & -690 & 0.069 & -0.065 \\
4192 & 2823 & -0.101 & -0.690
\end{array}\right)
$$

$$
M_{\text {NDEEXnoAcc }} \times 10^{3}=\left(\begin{array}{cccc}
5.05 & 17.9 & 5800 & 780 \\
0.953 & 0.335 & 2012 & 98 \\
-1390 & -690 & 19.2 & 0.823 \\
4290 & 2860 & -130 & -5.46
\end{array}\right) \text {. }
$$

The matrices are multiplied by $10^{3}$ for succinctness. As expected, there is a significant increase in the off-diagonal transfer matrix terms when the accelerating mode cavity is disabled, notably the $R_{65}$ and the $R_{55}$ terms.

Once we had working designs for both the DDEEX and NDEEX, we analyzed the performance for several cavity and accelerating models with each set of emittances, using the initial C-S parameters of $\alpha_{x, 0} \beta_{x, 0}=7.5,5.0 \mathrm{~m}$ respectively for the NDEEX and $\alpha_{x, 0}, \beta_{x, 0}=-5.0,28.2 \mathrm{~m}$ for the DDEEX, based on the results of parametric scans shown in the following section. 
Table 6.3: Quality of Emittance Exchange for various simulations, varying configuration, simulation order, model for TDC+ACC cavities, and which emittances/lengths are used ("2" uses shorter bunches and lower emittances than "1")

\begin{tabular}{cccccc}
\hline Simulation & Order & Emittance Type & Cav Model & $\mathcal{F}_{x \rightarrow z}$ & $\mathcal{F}_{z \rightarrow x}$ \\
\hline \hline DDEEX & 1 & 1 & Thin Matrix & 1.000 & 1.000 \\
DDEEX & 1 & 1 & RFDF+RFCA & 1.026 & 1.001 \\
DDEEX & 2 & 1 & Thin Matrix & 1.17 & 1.003 \\
DDEEX & 2 & 1 & RFDF+RFCA & 1.24 & 1.005 \\
\hline NDEEX & 1 & 1 & Thin Matrix & 1.000 & 1.000 \\
NDEEX & 1 & 1 & RFDF+RFCA & 1.000 & 1.000 \\
NDEEX & 2 & 1 & Thin Matrix & 1.003 & 1.001 \\
NDEEX & 2 & 1 & RFDF+RFCA & 1.003 & 1.001 \\
\hline \hline DDEEX & 1 & 2 & Thin Matrix & 1.000 & 1.000 \\
DDEEX & 1 & 2 & RFDF+RFCA & 1.006 & 1.000 \\
DDEEX & 2 & 2 & Thin Matrix & 1.045 & 1.000 \\
DDEEX & 2 & 2 & RFDF+RFCA & 1.066 & 1.000 \\
\hline NDEEX & 1 & 2 & RFDF+RFCA & 1.000 & 1.000 \\
NDEEX & 2 & 2 & RFDF+RFCA & 1.000 & 1.000 \\
\hline
\end{tabular}

For both configurations, the combination of a thin-cavity matrix and only firstorder effects results in perfect emittance exchange, while the smaller emittances and bunch lengths for the "A0"-scale emittances help reduce the emittance degradation from second-order effects. Likewise, the RFDF model contains second-order effects that are not accounted for in first-order matrix approximation, which results in the RFDF model giving worse numeric exchange.

The mitigation of higher-order effects, under which emittances are not preserved, are greatly important for the preservation of low emittances through any beamline. To determine operational parameters that minimize emittance growth, we perform scans of initial C-S parameters, $\beta_{x, 0}$ and $\alpha_{x, 0}$, in both the horizontal and vertical planes to find the optimal operating parameters for each of the different scenarios and configurations. For discussing the results of C-S scans, we define a general term, 
the acceptance, to define the range of C-S parameters that give good exchange of emittances. These are often chosen to best show the data presented in the simulations so that it is clearer to see the major conclusions. For the contour plots that represent the results of the C-S scans, we use a finite range of the $\mathrm{F}$ to display, so it is easier to discern the relative acceptances of different designs and configurations, as well as to show the gradient over acceptable emittance exchanges.

Units for the color scales are $\mu \mathrm{m}$ when presenting the normalized emittances, mm for RMS bunch sizes, and unitless when presenting the "exchange quality", $\mathcal{F}_{x \rightarrow z} \equiv \frac{\varepsilon_{z, f}}{\varepsilon_{x, 0}}$ and $\mathcal{F}_{z \rightarrow x} \equiv \frac{\varepsilon_{x, f}}{\varepsilon_{z, 0}}$, where perfect emittance exchange occurs at $\mathcal{F}=1$. In Figure 6.2, we demonstrate the quality of the emittance exchange as functions of the initial C-S parameters at using the NDEEX for several different scenarios to demonstrate the resulting higher-order effects and different TDC models.

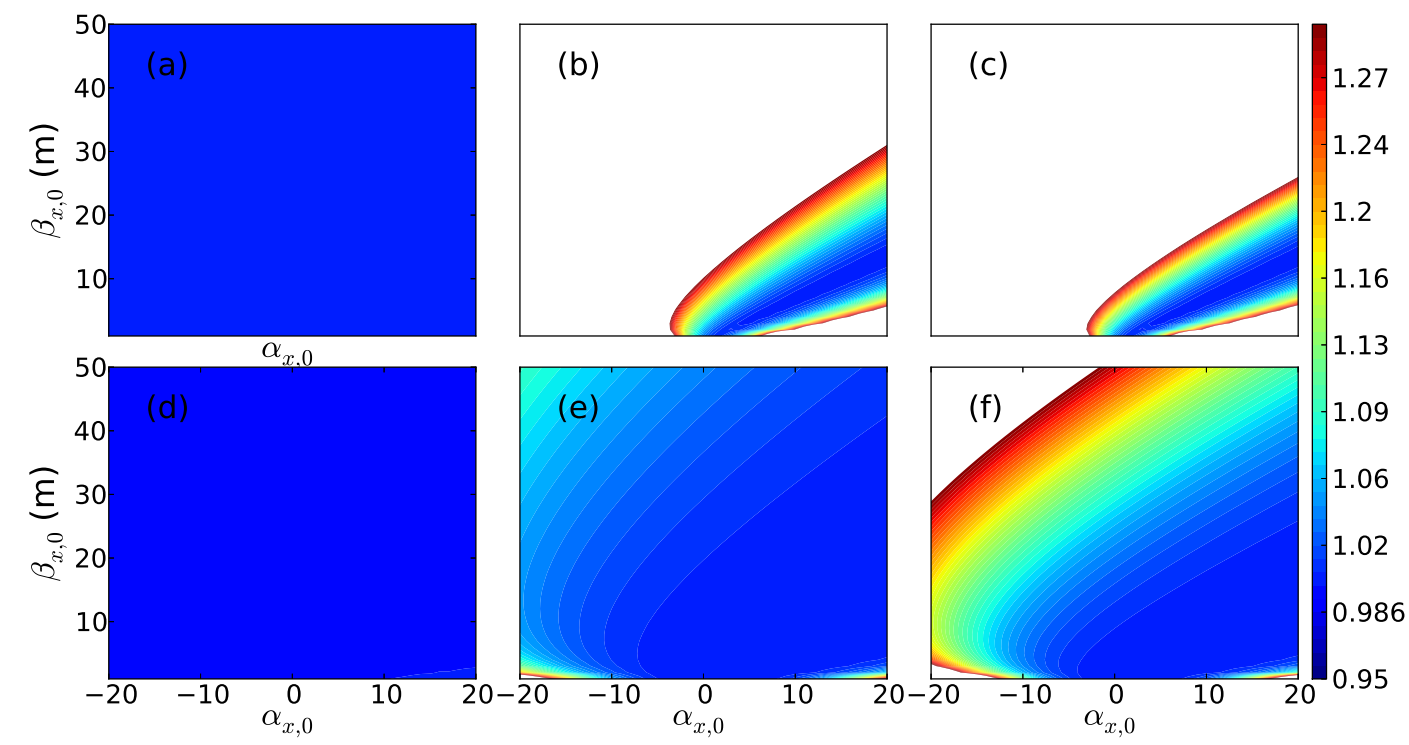

Figure 6.2: Contour plot of $\mathcal{F}_{x \rightarrow z}(\mathrm{a}, \mathrm{b}, \mathrm{c})$ and $\mathcal{F}_{z \rightarrow x}(\mathrm{~d}, \mathrm{e}, \mathrm{f})$ as a function of the initial $x$ C-S parameters for the NDEEX design with thin-cavity but without second order effects (a, d), second order effects with thin matrix TDC model (b, e), and with second order effects and RFDF model $(c, f)$. The addition of more second-order effects introduces and lowers the acceptance on the C-S parameters. 
Without a thin-matrix model for the TDC and only-first order effects, the quality of the emittance exchange is almost completely uniform over the range of C-S parameters that was scanned. When second-order effects are added for dipole magnets and quadrupole magnets, the quality of the emittance exchange starts to take the defining contour that is demonstrated in the majority of the C-S scans, which indicates the penalty to $\mathcal{F}$ associated with poor operating parameters. Finally, the RFDF+RFCA model is implemented, which introduces additional higher-order effects in the cavity fields, further restricting the acceptance for C-S parameters but still allowing for near-perfect exchange at the optimal operating parameters.

\subsection{DDEEX vs NDEEX in ELEGANT}

To compare the NDEEX and DDEEX designs, we must judiciously select fair operating parameters and geometry, as the two configurations have far different beam optics. We compare the core emittance exchanger designs for equivalent doglegs with dispersion of $\left|\eta_{x}\right|=0.5 \mathrm{~m}$. Figure 6.3 shows an initial comparison between the two configurations in ELEGANT using second-order effects and the RFDF TDC model.

The two configurations have far different optimal C-S parameters. The acceptance for the two configurations is comparable, but the DDEEX functions as a better bunch compressor, as can be noted due to its smaller $R_{51}$ terms, as portrayed in Eq. 6.13 compared to Eq. 6.15. The horizontal C-S ratio that minimizes the final bunch length is not the same ratio that has the best emittance exchange equality. For the chicane design, these are not far off from each other, while for the double dogleg design the ratios are in notably different directions. 

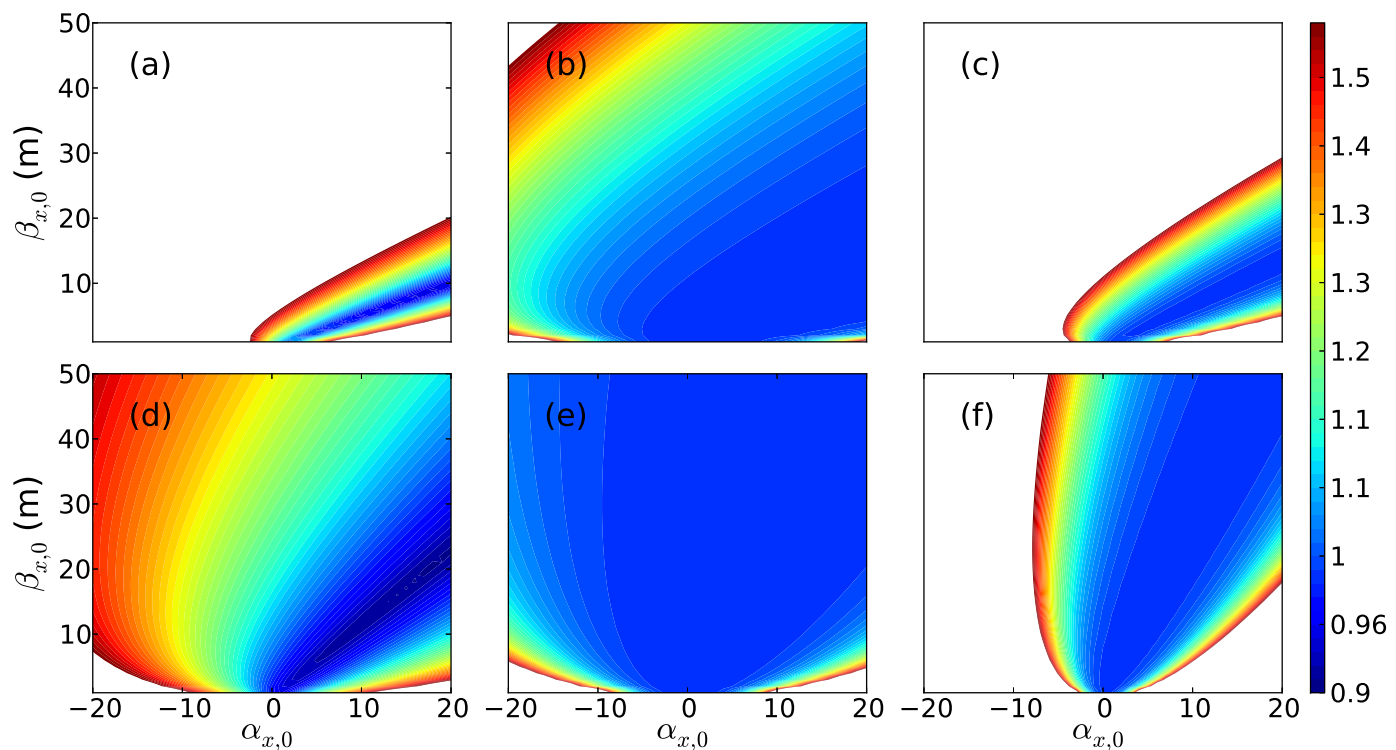

Figure 6.3: Contour plot of final bunch lengths $\sigma_{z}(\mathrm{a}, \mathrm{d}), \mathcal{F}_{x \rightarrow z}(\mathrm{~b}, \mathrm{e})$ and $\mathcal{F}_{z \rightarrow x}$ (c, f) as a function of the initial $x$ C-S parameters for the NDEEX (a,b,c) and DDEEX design (d,e,f), in ELEGANT, using the RFDF model and second order effects.

Second-order effects reduce the quality of the emittance exchange for both configurations and increase the dependence on beam optics. The first-order simulations, shown in Figure 6.2, are stable over a greater range, and the addition of higher-order effects is a stark degradation.

We also prefer a similar scan for the vertical $y$ C-S parameters, which shows far less interesting behavior due to the lack of interaction with the TDC or significant focusing-defocusing quadrupole magnets in this sense, the optics of the vertical plane are similar to that of traveling through a variable- $R_{56}$ bunch compressor. The results of the vertical scan are shown in Figure 6.4.

The valley of parameters that minimizes the final $y$ size falls within around $1.0 \leq$ $\frac{\alpha_{y}, 0}{\beta_{y}, 0} \leq 1.5$. We choose a value of 1.3 for the NDEEX and a value of -0.6 for DDEEX. For the geometry explored, the DDEEX maintains and produces a smaller beam size 


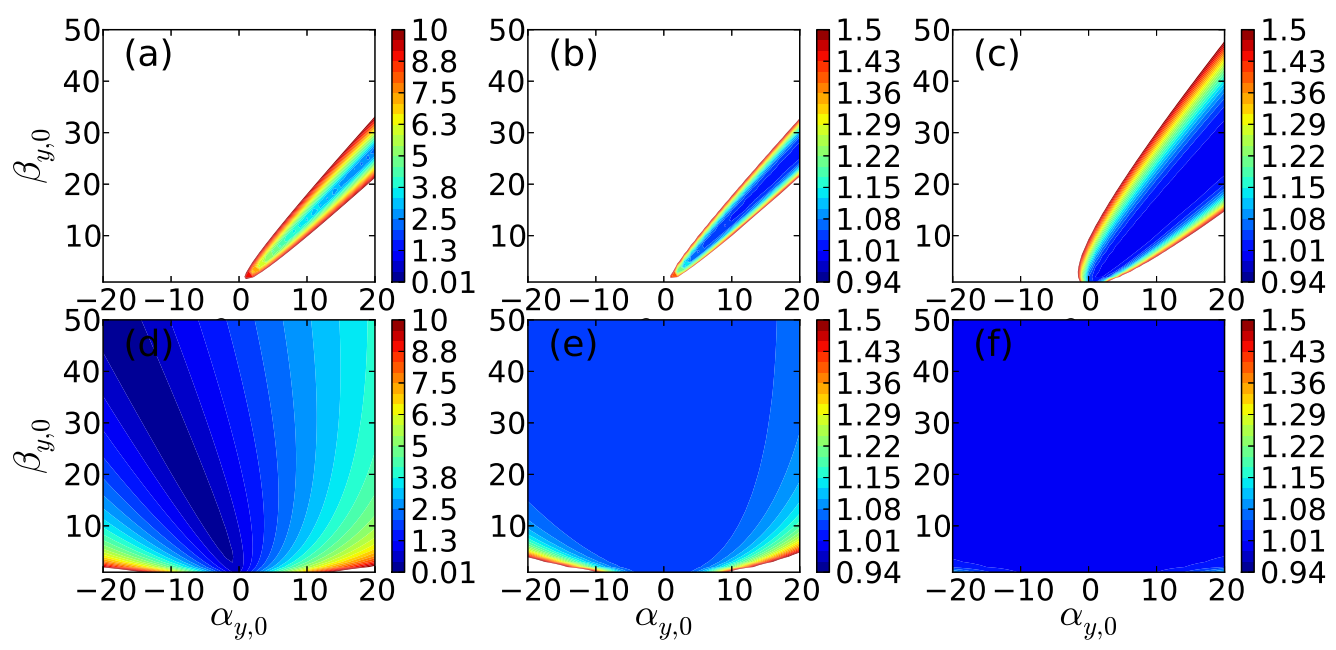

Figure 6.4: Contour plot of final vertical parameters $\sigma_{y}(\mathrm{a}, \mathrm{d}), \mathcal{F}_{x \rightarrow z}(\mathrm{~b}, \mathrm{e})$ and $\mathcal{F}_{z \rightarrow x}$ $(\mathrm{c}, \mathrm{f})$ as a function of the initial $y \mathrm{C}-\mathrm{S}$ parameters for the NDEEX (a-c) and DDEEX (d-f) designs.

Table 6.4: Optimal $\frac{\alpha_{x, 0}}{\beta_{x, 0}}$

\begin{tabular}{ccc}
\hline Simulation & Optimal for $\sigma_{z}$ & Optimal for $\varepsilon_{z}$ \\
\hline \hline NDEEX & 2.0 & 1.5 \\
DDEEX & 0.8 & 0.4 \\
\hline
\end{tabular}

in $y$, and bears relatively little influence on the exchange ratio, unlike the vertical parameters of the NDEEX.

It is beneficial to choose an operating parameter and EEX configuration that allows for both the creation of upright beams as well as the minimization of longitudinal emittance. However, this may be dictated by the design, as the two regions do not naturally overlap each other for any of the designs studied here, and, as we show later, are strongly influenced by CSR. We make note of the C-S parameters that lead to the minimization of final bunch length, $\sigma_{z}$, and the exchange quality $\mathcal{F}_{x \rightarrow z}$, in Table 6.4 . 


\subsection{Comparative Studies in IMPACT-Z}

We repeat the methodology for C-S scans presented in the previous section in the simulation code IMPACT-Z. Simulations were performed without collective effects, and then with collective effects. Here, we explore the boosted-dispersion configuration using $\eta_{-}=1.0 \mathrm{~m}$; greater values are explored in a later section. Note that for highly divergent beams, such as those in the lower corners of some C-S scans, there is significant particles loss in IMPACT-Z and the simulations fail to complete without error. We zero-out these simulations, which fall within the white regions in the lower corners of some IMPACT-Z plots. Comparisons to the ELEGANT simulations indicate that these entirely fall within unacceptable regions of poor emittance quality. The exchange qualities $F_{z \rightarrow x}$ and $F_{x \rightarrow z}$ are shown in Figs. $6.5 \& 6.6$, respectively, for both the NDEEX and DDEEX, using ELEGANT and several different IMPACT-Z.

As the transverse and longitudinal dynamics change depending on the initial C-S parameters, an example of the different dynamics for four sets of C-S parameters are presented in Figure 6.7. The final bunch length is controlled by the initial C-S parameters, while the final $\sigma_{x}$ is dominated by the kick from the TDC. This can be controlled by altering the initial energy chirp to the beam, as it controls the bunch length at the TDC. However, increasing the chirp to achieve compression and a smaller final bunch length also makes the transverse beam size larger in the first dogleg and at the TDC location, due to the energy dispersion. This introduces increased second-order degradation from the upstream beamline. This demonstrates the complex nature of trade-offs in the selection of operating parameters.

In Figure 6.8, the DDEEX, NDEEX, and BDEEX are compared for the case of no collective effects. Boosting the dispersion causes a notable decrease in the 

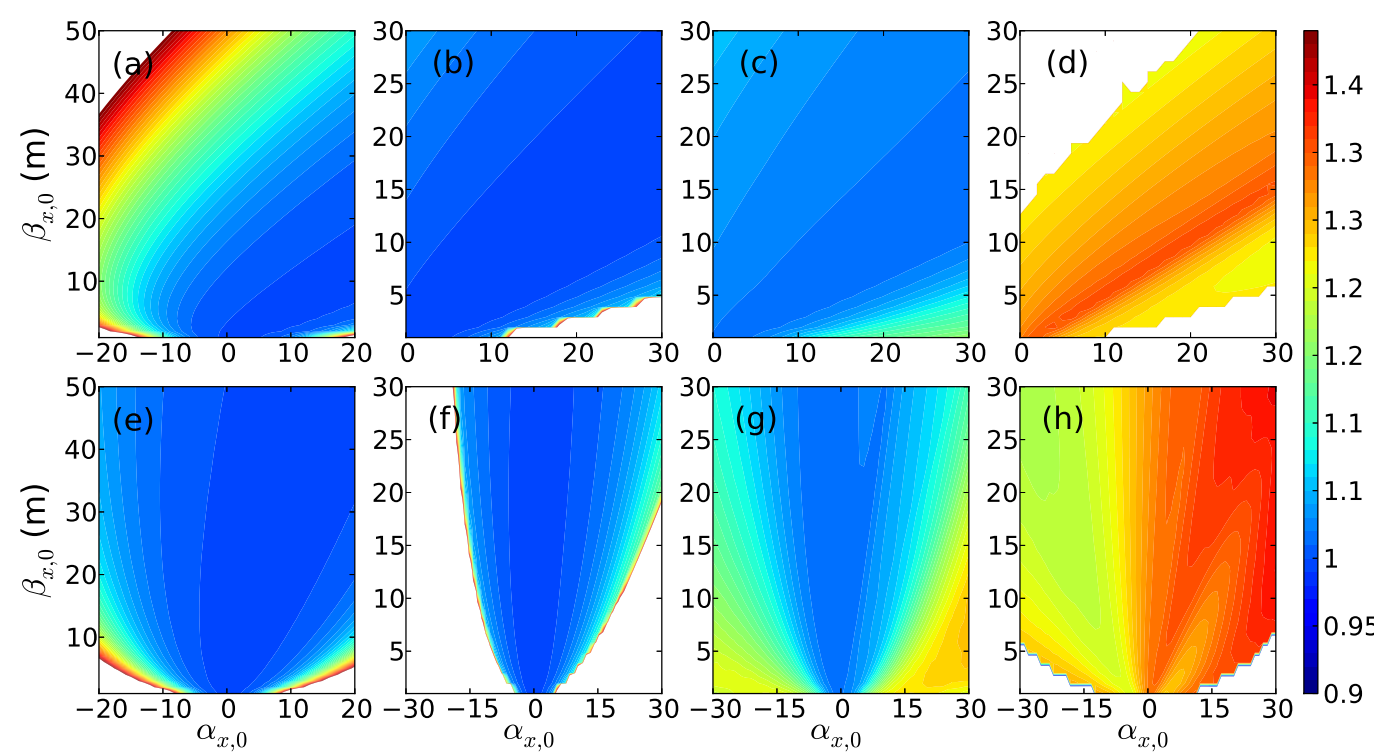

Figure 6.5: Contour plots of $F_{z \rightarrow x} \operatorname{NDEEX}(\mathrm{a}-\mathrm{d})$ and DDEEX (e-h) designs in ELEGANT (a,e) and Impact-Z for $0 \mathrm{nC}(\mathrm{b}, \mathrm{f}), 1 \mathrm{nC} \mathrm{SC}(\mathrm{c}, \mathrm{g})$, and $1 \mathrm{nC} \mathrm{SC}+\mathrm{CSR}(\mathrm{d}, \mathrm{h})$ as functions of the C-S parameters at the EEX entrance, $\beta_{x, 0}$ and $\alpha_{x, 0}$. Note the different scales for $\alpha$, which is dictated by region of interest as determined by prior simulations.

acceptance of initial C-S parameters, as the quadrupole magnet settings within the chicane cause the transverse beam sizes to be more erratic along the beamline.

When collective effects are introduced, as shown in Figure 6.9, the acceptance is reduced for all three configurations. Comparisons of $\sigma_{z}$ to the contour plots for the emittance exchange quality reveal a significant degradation to the emittance exchange quality that occurs when the beam size is at its minimum, i.e., when the final LPS is upright, a vital part of achieving optimal beam shaping.

This indicates a significant barrier to achieving optimal shaping while also achieving optimal emittance exchange, and that there is some significant trade-off that must be determined when setting the EEX settings. 

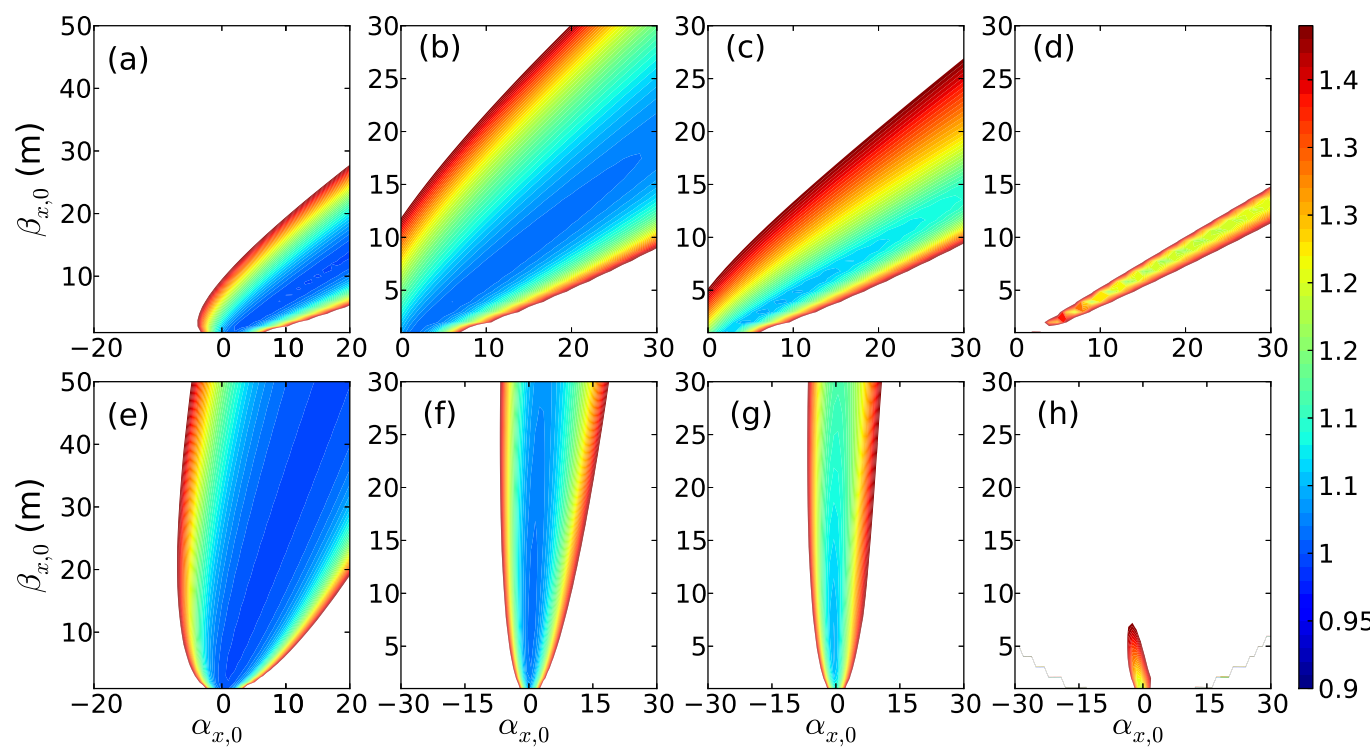

Figure 6.6: Contour plots of $\mathcal{F}_{x \rightarrow z} \operatorname{NDEEX}(\mathrm{a}-\mathrm{d})$ and DDEEX (e-h) designs in ELEGANT (a,e) and Impact-Z for $0 \mathrm{nC}(\mathrm{b}, \mathrm{f}), 1 \mathrm{nC} \mathrm{SC}(\mathrm{c}, \mathrm{g})$, and $1 \mathrm{nC} \mathrm{SC}+\mathrm{CSR}(\mathrm{d}, \mathrm{h})$ as functions of the C-S parameters at the EEX entrance, $\beta_{x, 0}$ and $\alpha_{x, 0}$. Note the different scales for $\alpha_{x}$, which is dictated by region of interest as determined by prior simulations.

To study the effects of space charge, we use the Debye length, which is given by

$$
\lambda_{D}(s)=\frac{\varepsilon_{x, y}(s)}{2} \sqrt{\gamma(s) \beta(s) \frac{I_{A}}{I(s)}}
$$

where $\gamma$ and $\beta$ are the relativistic parameters, $I_{A}$ is the Alfvren current of $17 \mathrm{kA}$, and $I(\mathrm{~s})$ is the current of the bunch, we we take as the bunch charge divided by $\sqrt{5}$, which is an approximation for a Gaussian bunch. When $\lambda_{D}>>\sigma_{x, y}$, the beam is said to be space-charge dominated, while if $\lambda_{D}<<\sigma_{x, y}$ the beam is said to be emittance dominated. We analyze IMPACT-Z SC+CSR simulations in Figure 6.10 to demonstrate the shift in regimes. 


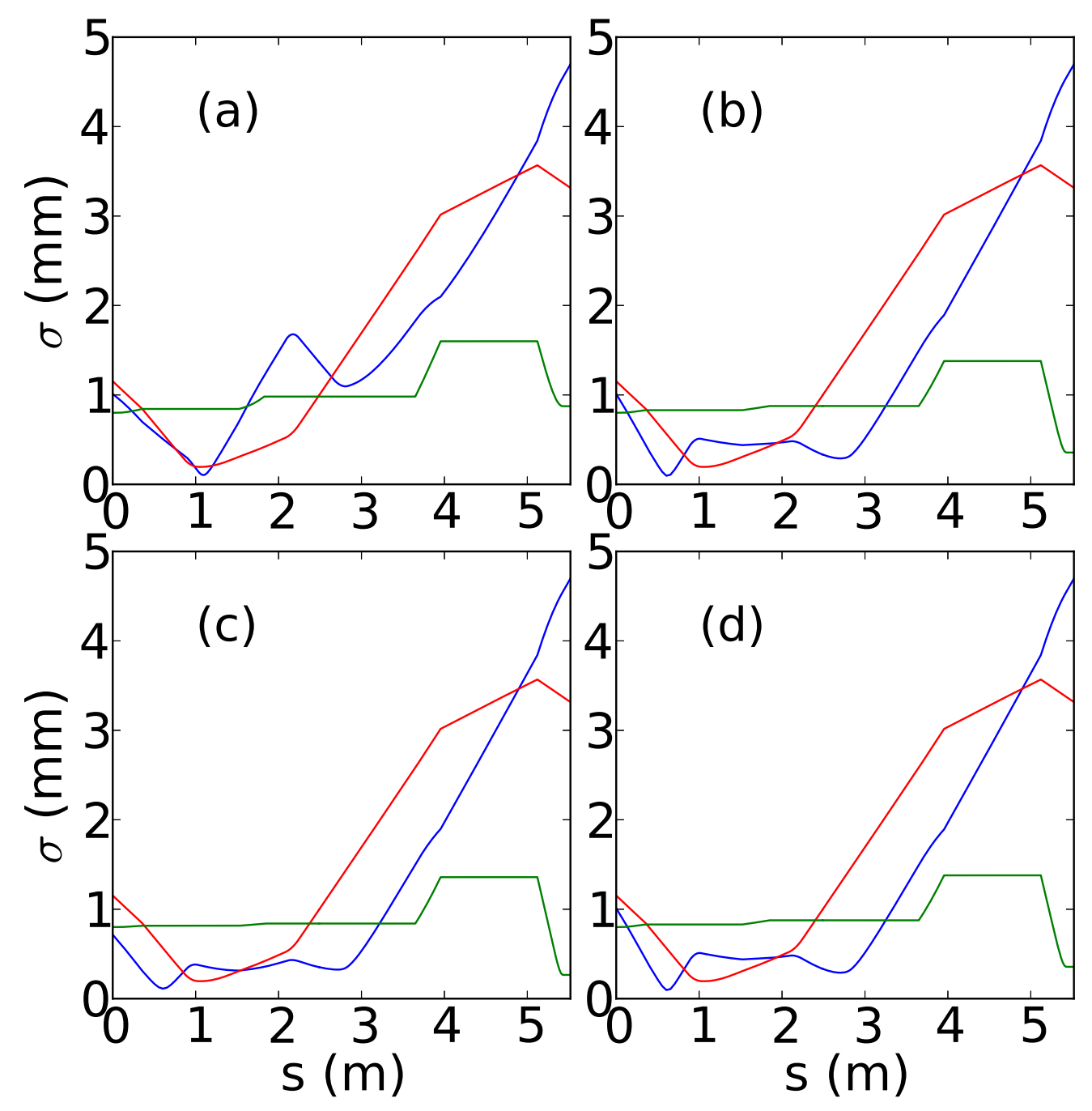

Figure 6.7: Horizontal (blue), vertical (green), and longitudinal (red) RMS beam sizes along the EEX for C-S parameters of $\alpha_{x, 0}, \beta_{x, 0}=7.5,10 \mathrm{~m}$ (a), 15,10 m (b),

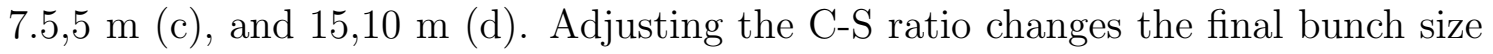
and, due to second order effects, the overall quality of the emittance exchange.

When the Debye length is analyzed, we can see that beam transitions from an emittance dominated regime to a space-charge dominated regime after traveling through the emittance exchanger.

Figs. 6.2-6.9 show the range of acceptable parameters. The overall band of acceptable values is of great use when considering other shaping conditions, as is 

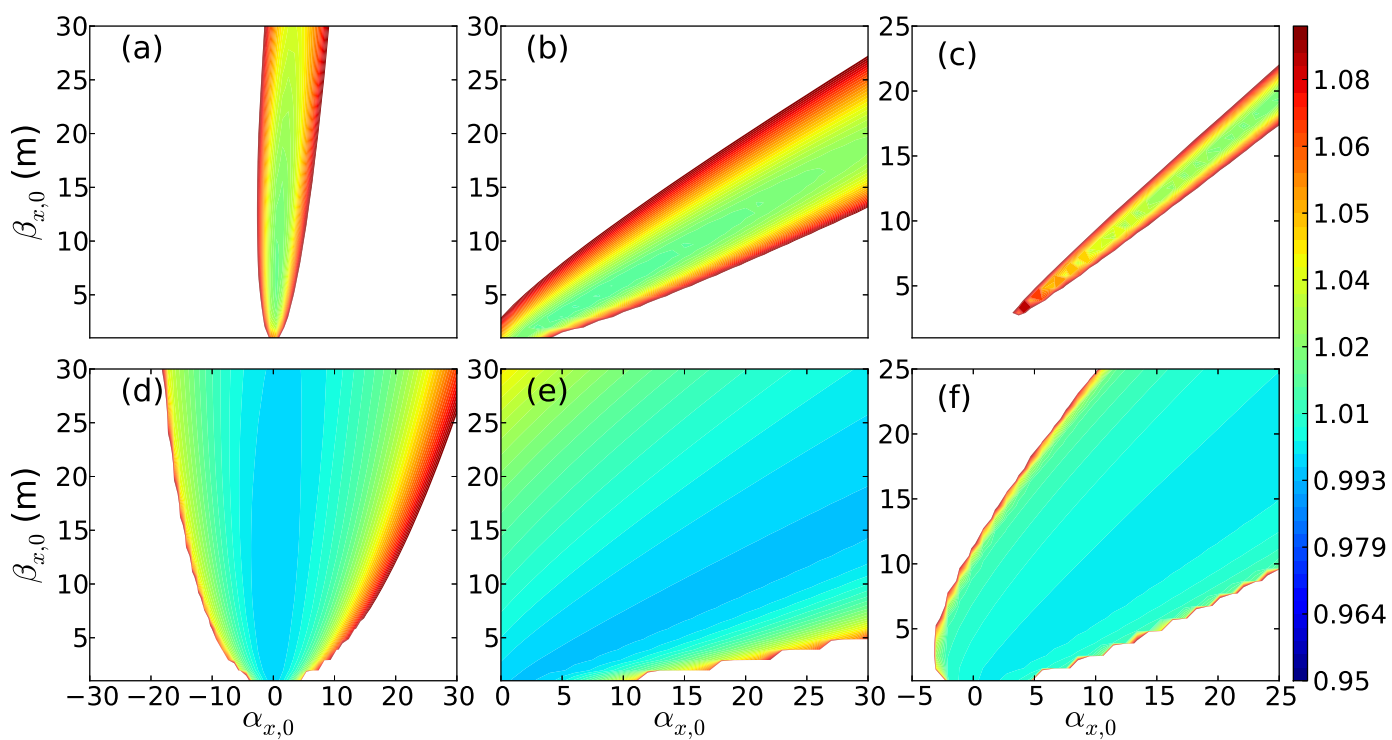

Figure 6.8: Contour plots comparison of three models (DDEEX (a,d), NDEEX $(\mathrm{b}, \mathrm{e})$, and $\operatorname{BDEEX}(\mathrm{c}, \mathrm{f}))$ with IMPACT-Z for $0 \mathrm{nC}$ for both $\mathcal{F}_{x \rightarrow z}$ (a-c) and $\mathcal{F}_{z \rightarrow x}$ (d-f), as functions of the C-S parameters at the EEX entrance, $\beta_{x, 0}$ and $\alpha_{x, 0}$. Note the different scales for $\alpha$, which is dictated by region of interest as determined by prior simulations.

discussed in the following section. In a perfectly linear system, all of the designs and TDC models, with the appropriate $R_{65}$ correction, give perfect emittance exchange, which is well-demonstrated in our simulations.

The chicane design appears to have a larger acceptance of C-S parameters than the double dogleg design. This is best demonstrated by Figures 6.5 and 6.6. When limiting the range of acceptable $\mathrm{F}$ to be under 1.4, with $1 \mathrm{nC}$ and $\mathrm{SC}+\mathrm{CSR}$ enabled, the DDEEX's acceptance for $\mathcal{F}_{x \rightarrow z}$ is small, but still allows for region of good exchange. 


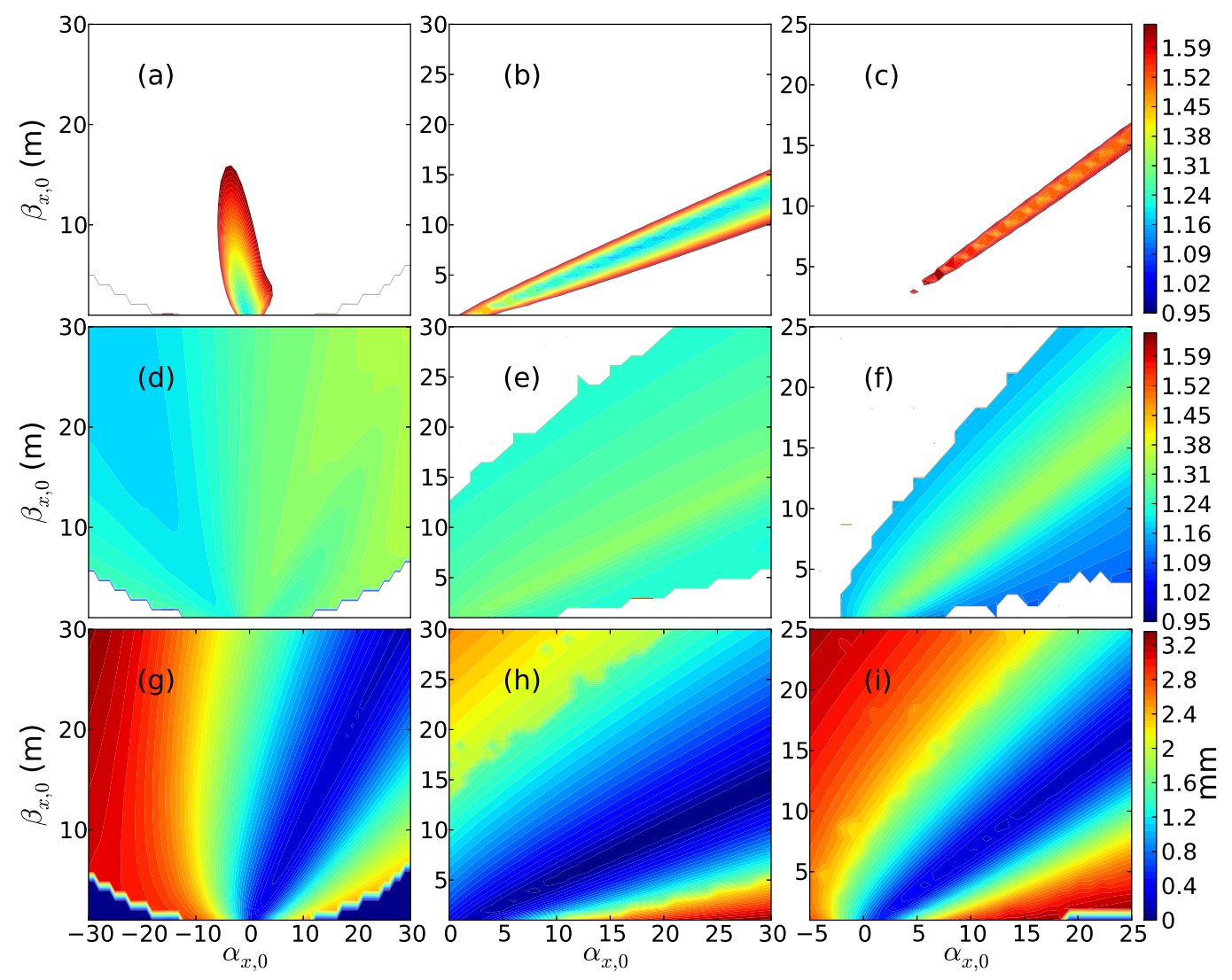

Figure 6.9: Contour plots comparison of three models (DDEEX, NDEEX, and BDEEX) with IMPACT-Z for $0 \mathrm{nC}$ for both $\mathcal{F}_{z x}$ and $\mathcal{F}_{x z}$, as functions of the C$\mathrm{S}$ parameters at the EEX entrance, $\beta_{x, 0}$ and $\alpha_{x, 0}$. Note the different scales for $\alpha$, which is dictated by region of interest as determined by prior simulations. 

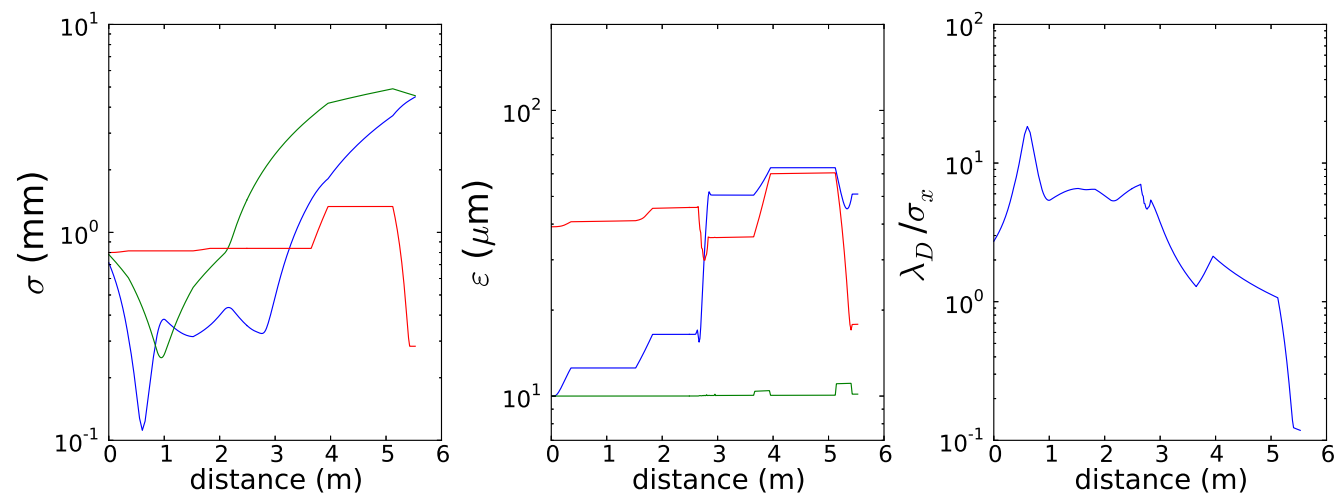

Figure 6.10: Bunch size (left), emittances (middle) and Debye length over RMS bunch size (right) for the NDEEX design simulated with IMPACT-Z with 1nC $\mathrm{SC}+\mathrm{CSR}$, with $x$ (blue), $y$ (red) and $z$ (green) plotted together.

\subsection{Code Benchmarking}

In addition to the detailed simulation study performed with ELEGANT and IMPACT-Z, simulations with CSRTRACK were performed for limited cases. Unlike the other two codes, CSRTRACK has neither a built-in model of a transverse deflecting cavity nor the capability of using user-defined scripts. To simulate the TDC, the NDEEX design was split in two halves and linked together using GLUETRACK, with a thin-cavity model implemented as a matrix between the two sections. In Table 6.5, the results of the CSRTRACK simulations are presented in comparison to Elegant and Impact-Z simulations using the same initial beam parameters, using CSRTRACK's particle-to-particle (P2P) model, its 1-D projected (1DP) model of CSR, and its model without collective effects.

In the absence of collective effects, the emittance exchange in CSRTRACK is perfect, which verifies the geometry and functionality of the CSRTRACK model. With collective effects, the emittance exchange becomes considerably worse. Even with only the 1DP model, the exchange is worse than the IMPACT-Z studies. 
Table 6.5: Exchange quality for various simulations for the NDEEX, at $\beta_{x, 0}=5 \mathrm{~m}$ and $\alpha_{x, 0}=7.5$.

\begin{tabular}{cccc}
\hline Simulation & Bunch Charge $(\mathrm{nC})$ & $\mathcal{F}_{x \rightarrow z}$ & $\mathcal{F}_{z \rightarrow x}$ \\
\hline \hline Elegant & - & 1.0 & 1.0 \\
Impact-Z SC+CSR & 1.0 & 1.3 & 1.3 \\
CSRtrack & 0.0 & 1.0 & 1.0 \\
CSRtrack 1DP & 1.0 & 1.7 & 1.9 \\
CSRtrack P2P & 1.0 & 1.6 & 2.3 \\
\hline
\end{tabular}

\subsection{Dispersion Boosting}

Boosting the dispersion up to $6 \mathrm{x}$ of the nominal value (in this case, $3.0 \mathrm{~m}$ ) introduces significant complications to beam control. As the $x$ transfer matrix in the second dogleg must be controlled to specifically satisfy the basic requirements for perfect emittance exchange, the $y$ dynamics becomes difficult to control. For an example of optimized function, see Figure 6.11, using both ASTA and A0-style beam emittances.

The key difference between these simulations and those of the nominal-dispersion and double-boosted-dispersion is that $\sigma_{y}$ is large and divergent, in addition to $\sigma_{x}$, which is large and divergent due to the kick from the TDC. Due to the focusingdefocusing nature of quadrupole magnets, it is easier to deal with one large-anddiverging transverse dimension than it is to deal with both together, as shown in Figre 6.12. This EEX design becomes more feasible for smaller emittances, due to the beam size being smaller, though $\beta_{y}$ will still grow considerably.

The key feature of dispersion boosting is the reduced requirements on the TDC, so while the quadrupole magnet strengths are increased, the transverse beam size is less dominated by the TDC kick. The trade-offs that must be considered are not 

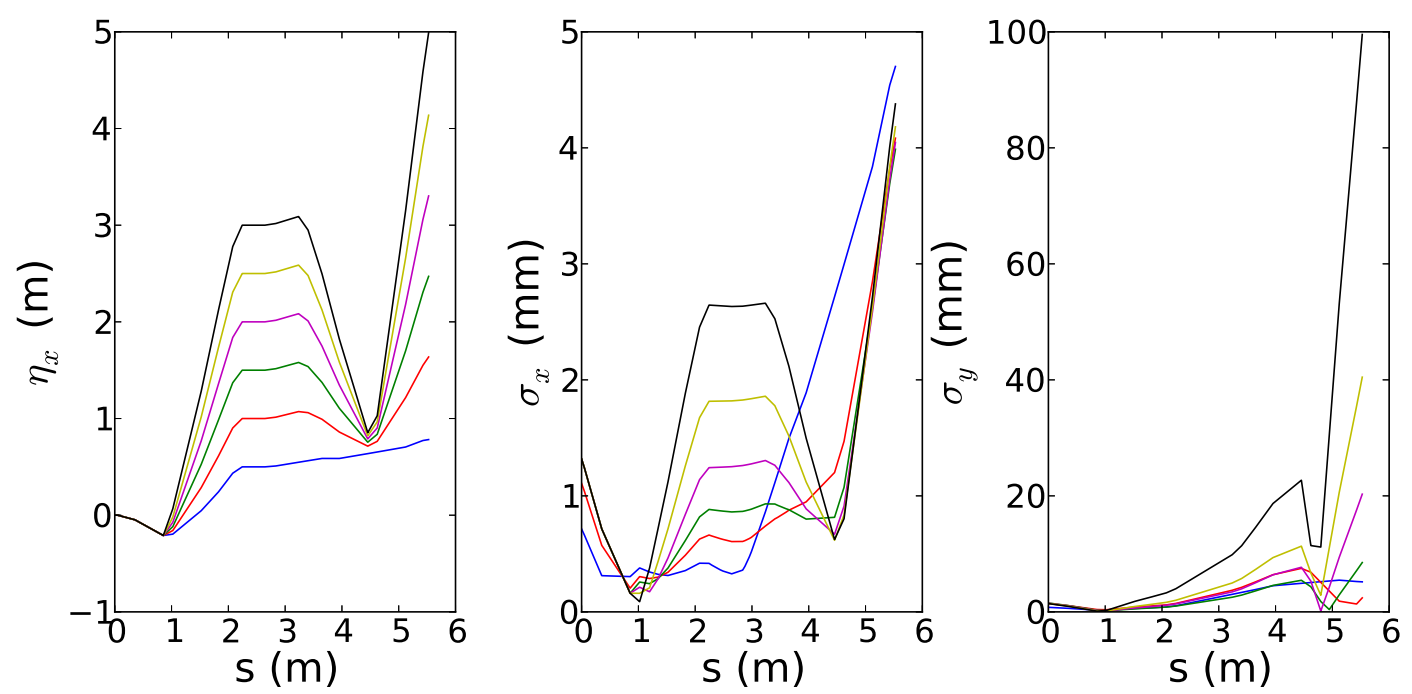

Figure 6.11: Horizontal dispersion $\eta_{x}$ (left), $\sigma_{x}$ (middle), and $\sigma_{y}$ (right) along the NDEEX and BDEEX designs for $\eta_{-}=0.5 \mathrm{~m}$ (blue), $1.0 \mathrm{~m}$ (red), $1.5 \mathrm{~m}$ (green), $2.0 \mathrm{~m}$ (magenta), $2.5 \mathrm{~m}$ (yellow) and $3.0 \mathrm{~m}$ (black). In the absence of higher-order effects, each of the emittance qualities are near $\mathrm{F}=1$. However, as the quadrupole magnets inside the EEX are adjusted to control the dispersion and $x-z$ transfer matrix, the vertical beam size becomes increasingly less-constrained, with the $3.0 \mathrm{~m}$ case being highly divergent and unusable.

simple, and depend greatly on both initial bunch length and transverse emittances. Dispersion-boosting up to $3 \mathrm{x}$ of the baseline dispersion is possible with ASTA-scale beam parameters, but greater dispersion is feasible for lower emittance regimes. The emittance exchange quality for various levels of dispersion boosting is shown in Table 6.6.

\subsection{Shaping Studies}

Quadrupole magnets placed upstream of the first dipole magnet of the EEX can be used to control the overall $R_{51}$ and $R_{52}$ of the emittance exchanger system. Setting $R_{52}$ to zero makes the initial transverse distribution translate over exactly 

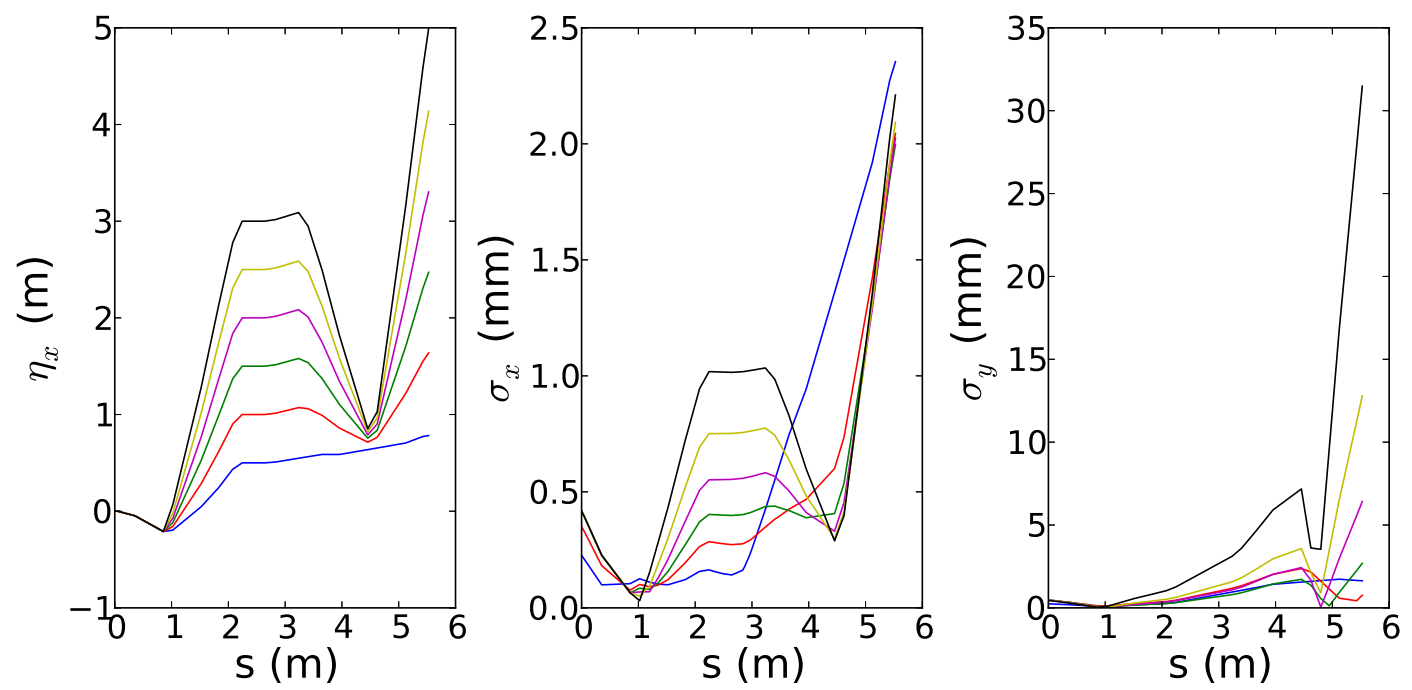

Figure 6.12: Horizontal dispersion $\eta_{x}$ (left), $\sigma_{x}$ (middle), and $\sigma_{y}$ (right) along the NDEEX and BDEEX designs for $\eta_{-}=0.5 \mathrm{~m}$ (blue), $1.0 \mathrm{~m}$ (red), $1.5 \mathrm{~m}$ (green), $2.0 \mathrm{~m}$ (magenta), $2.5 \mathrm{~m}$ (yellow) and $3.0 \mathrm{~m}$ (black). In the absence of higher-order effects, each of the emittance qualities are near $\mathrm{F}=1$, using the smaller set of emittances.

into a current profile, with $R_{51}$ acting a scaling factor. This is useful for shaping ramped pulses for dielectric wakefield acceleration [6].

With the previous discussion on the optimal ratio, we can come up with a set of parameters that should be met. There are four key goals that must be met for shaping. First, the horizontal C-S ratio must allow for a reasonable proximity of perfect exchange, i.e., small enough to mitigate second-order effects. Second, the vertical C-S ratio must allow for a reasonable proximity of perfect exchange, i.e., keeps the beam small. This could be achieved by setting a maximum value for the transverse beam size, but the $\mathrm{C}-\mathrm{S}$ scans in the previous section reveal appropriate range rather than specific values. Third, $R_{52}=0$, for shaping, such that the fourth condition, control of $R_{51}=\mathrm{D}$, can act as a stretching factor for exact translation of the transverse projection into a longitudinal current profile. 
Table 6.6: Quality of Emittance Exchange for various dispersions using the BDEEX configuration, along with which emittances were used ("A0" is shorter bunch and lower emittance than "ASTA")

\begin{tabular}{cccc}
\hline$\eta_{-}(\mathrm{m})$ & Beam Type & $\mathcal{F}_{x \rightarrow z}$ & $\mathcal{F}_{z \rightarrow x}$ \\
\hline \hline 0.5 & 1 & 1.00 & 1.00 \\
0.5 & 2 & 1.00 & 1.00 \\
1.0 & 1 & 1.04 & 1.02 \\
1.0 & 2 & 1.01 & 1.00 \\
1.5 & 1 & 1.08 & 1.01 \\
1.5 & 2 & 1.01 & 1.000 \\
2.0 & 1 & 2.407 & 1.05 \\
2.0 & 2 & 1.22 & 1.000 \\
2.5 & 1 & 10.42 & 1.12 \\
2.5 & 2 & 3.43 & 1.01 \\
3.0 & 1 & 79.98 & 1.75 \\
3.0 & 2 & 25.35 & 1.04 \\
\hline
\end{tabular}

In addition, we must make some assumptions regarding the beam that is incoming to the shaping telescope. The beam dynamics and transfer matrices are over-constrained, and they need to be prioritized and weighted appropriately.

With these conditions considered, we achieve compression of $R_{51}=0.8$, while keeping the beam constrained reasonably well and $R_{52}<0.001 \mathrm{~m}$. As shown in Figure 6.13, collimation from $3.2 \mathrm{nC}$ to $1.0 \mathrm{nC}$, with reasonable C-S parameters, can result in a well-formed ramped bunch with a total bunch length on the order of $1 \mathrm{~mm}$, which is ideal for a possible dielectric wakefield experiment that is planned for ASTA.

For the transformation shown in Figure 6.13, the emittance exchanges are 1.0 and 1.07, respectively. This improves considerably when the fitting requirements are loosened, such as disregarding specific fitting of $R_{51}$. In Figure 6.14, we show the effects of collective effects on the final current profile. 

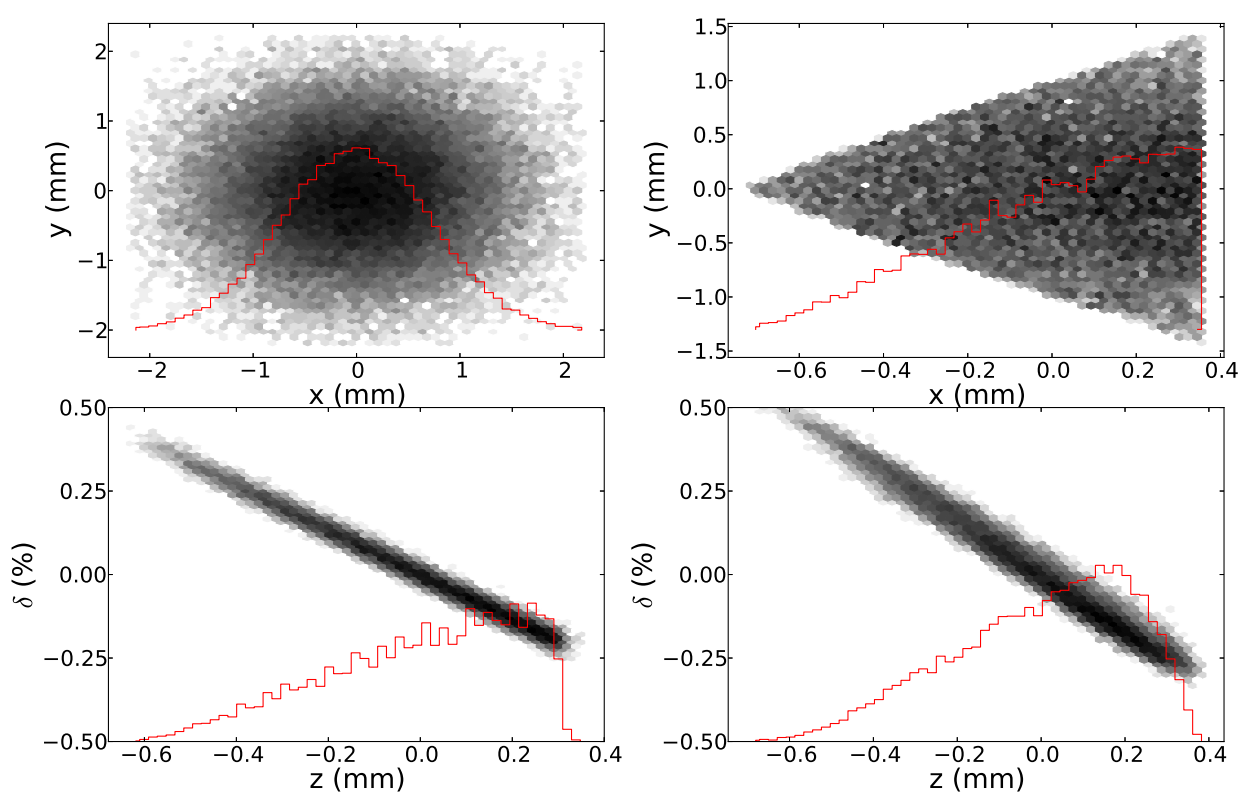

Figure 6.13: Pre-collimated (Upper Left), post-collimated (Upper Right) transverse profile, and post-EEX LPS without (lower left) and with lower right) collective effects, with projection of the $x$-axis shown in red. The collimation reduces the bunch charge from $3.2 \mathrm{nC}$ to $1.0 \mathrm{nC}$, and is based on a triangular shape with a slight exponential modulator such that it can cut the initial Gaussian into a triangular profile.

Generally, the introduction of collective effects reduces how well-defined the transported ramped bunch is by increasing the length of the tail. For a microbunch train, the projected length of each microbunch is increased, and beamlets become less well-defined, similar to the result of second-order effects that are presented earlier in this paper.

\subsection{High Energy Variant}

At ASTA there are two possible locations and energies for the emittance exchanger. There is the low-energy experimental area at $50 \mathrm{MeV}$, and downstream of 


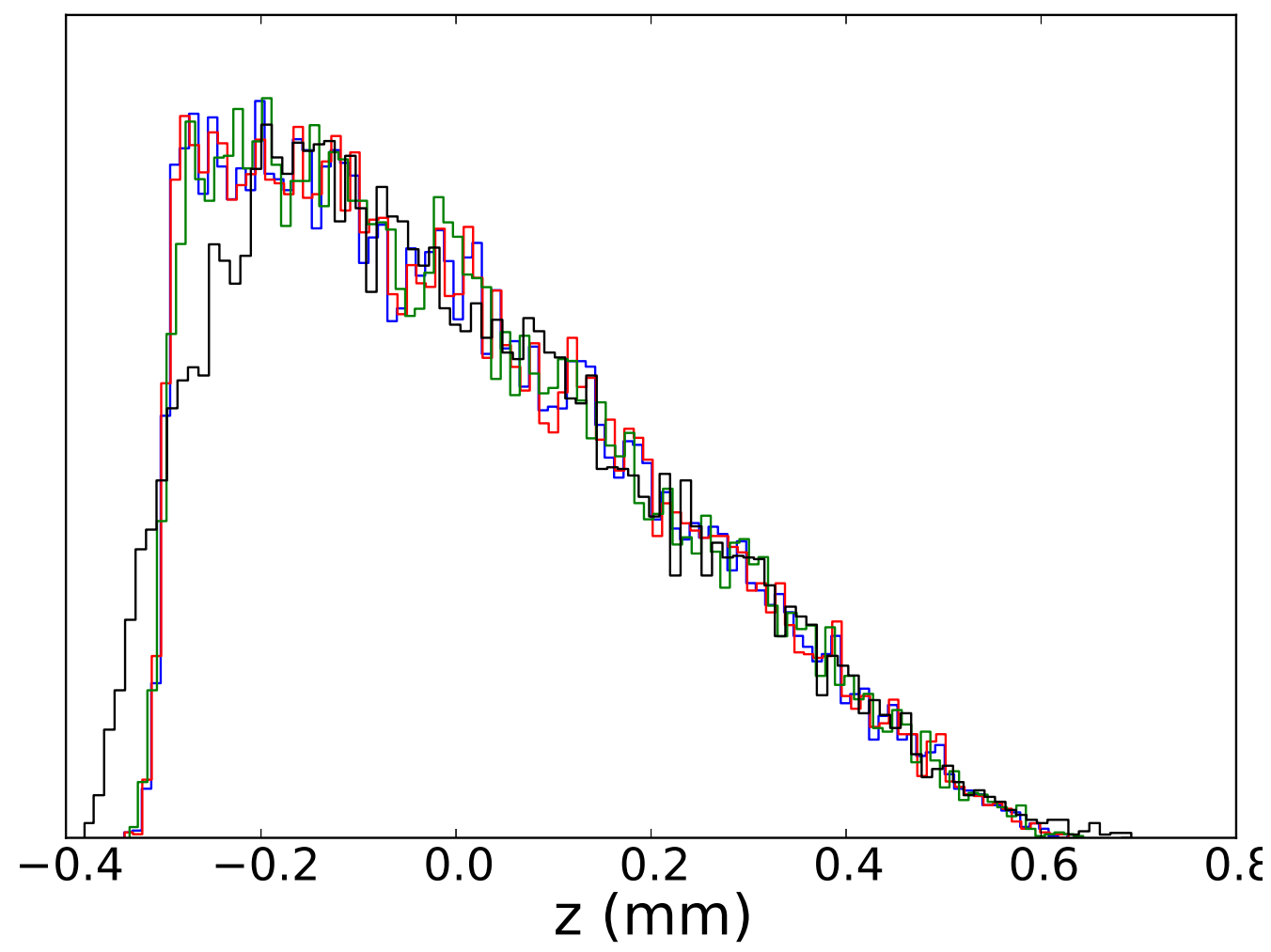

Figure 6.14: Post-EEX normalized density profiles for various levels of collective effects. The same bunch is tracked through IMPACT-Z with the level of SC and CSR adjusted to emulate various charges, with $0 \mathrm{nC}$ (red), $20 \mathrm{pC}$ (blue), $250 \mathrm{pC}$ (green) and $1.0 \mathrm{nC}$ (black). The hard edge drop-off in current at the tail of the bunch smooths out and elongates, at the expense of peak-density, as bunch charge increases.

the first cryomodule, as well as nominally $250 \mathrm{MeV}$, though it may be achieved in the downstream beamline at $50 \mathrm{MeV}$ by passing the beam through the cryomodule without acceleration. We perform C-S scans using a $250 \mathrm{MeV}$ beam similar to those demonstrated in the previous section, the results of which are shown in Figure 6.15.

Increasing the beam energy from $50 \mathrm{MeV}$ to $250 \mathrm{MeV}$ also increases the requirements on the TDC, which will require more power and associated cooling. We hope to counterbalance this with dispersion boosting, as discussed in the previous section. 

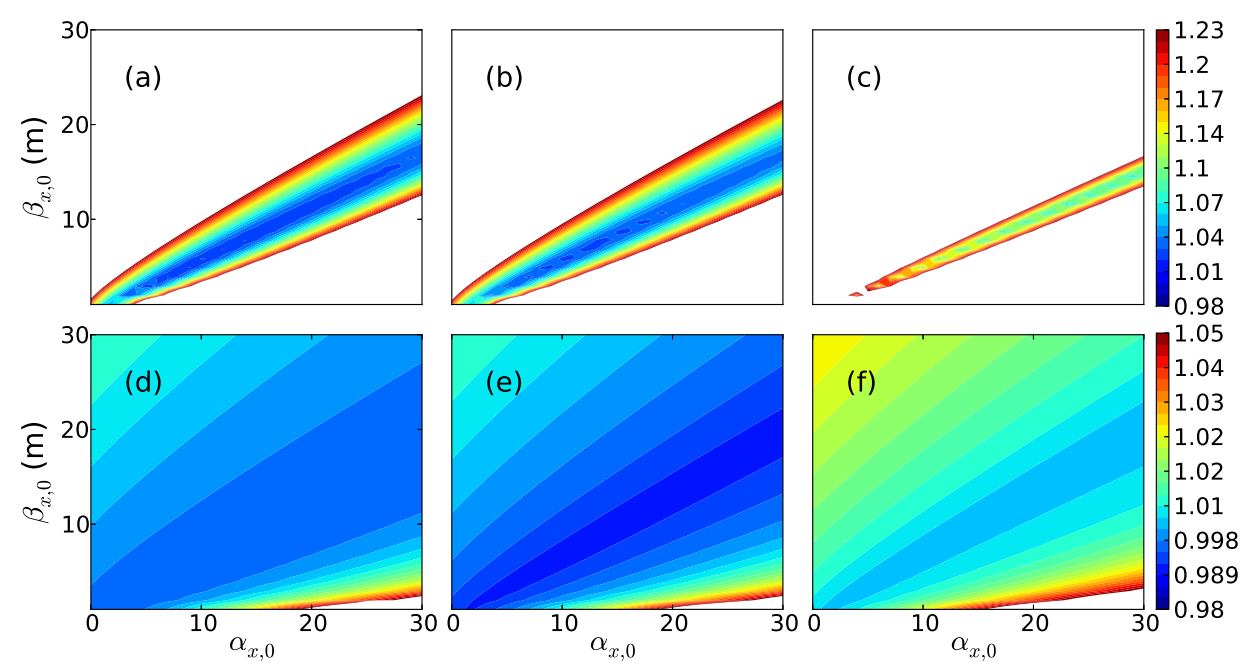

Figure 6.15: Contour plots comparison with IMPACT-Z for $0 \mathrm{nC}(\mathrm{a}, \mathrm{d}), 1 \mathrm{nC} \mathrm{SC}(\mathrm{b}, \mathrm{e})$, and $1 \mathrm{nC} \mathrm{SC+CSR}(\mathrm{c}, \mathrm{f})$ with both $\mathcal{F}_{x \rightarrow z}(\mathrm{a}-\mathrm{c})$ and $\mathcal{F}_{z \rightarrow x}(\mathrm{~d}-\mathrm{f})$, as functions of the C-S parameters at the EEX entrance, $\beta_{x, 0}$ and $\alpha_{x, 0}$, at a beam energy of $250 \mathrm{MeV}$.

CSR introduces a significant reduction in the exchange quality to $\mathcal{F}_{x \rightarrow z}$, more so than the change to $\mathcal{F}_{z \rightarrow x}$. This is because the bunch length is reduced in the second-dogleg relative to the first, which drives increased CSR, which increases the $\varepsilon_{z}$ more than it increases $\varepsilon_{x}$, though both are increased by longitudinal CSR due to its occurence during dispersive regions.

\subsection{Double Emittance Exchanger}

Lastly, we explore a Double Emittance Exchanger (2XEEX), which is two emittance exchangers placed in sequence with connecting optics to allow the exchange of longitudinal-to-transverse, then manipulate in the transverse plane, before exchanging back into the longitudinal.

In the long term, ASTA may also include a double emittance exchanger [74] and simulation studies are underway; an early design is shown in Figure 6.16. Second- 
order effects in particular are a significant obstacle to operation of a double EEX. The downside to the 2XEEX design is that keeping the beam constrained is far more difficult than the single EEX design.

For the 2XEEX, we use a telescope of four quadrupole magnets placed between the two emittance exchangers to match the out-coming beam of EEX1 to C-S parameters desirable for EEX2. This is a very constrained system. The concerns of keeping 2nd order effects reduce how much compression can be achieved. If the modulation is compressed in $x$ (as in Reference [74]) or in $x^{\prime}$, then the beam divergence or size increases proportionally, respectively. These approaches have the downsides of using the telescope. So, while these two scenarios are the simplest due to them presenting a clear conceptual way in which the overall compression is achieved, their neglect of the vertical dimension causes concerns regarding the blowup of $\sigma_{y}$. A mix of the two, with the initial longitudinal modulation exiting EEX1 with some overall correlation in the $x$ phase space, may be easier to control and compress, and may be a viable alternative to the earlier proposals.

For testing a 2XEEX, we create an initial bunch train, where each microbunch has some energy chirp, which is based through the NDEEX studied throughout this paper. We then develop a series of fit parameters to be solved with ELEGANT's optimizing functions. First, we aim for control of the horizontal C-S parameters, $\frac{\alpha_{x}, 0}{\beta_{x}, 0}=\frac{R_{51}}{R_{52}} \simeq 1.5$, such that the final beamlets have an upright LPS at the exit of EEX2. Then, the vertical C-S parameters must be controlled so that the $y$ beam envelope is well-behaved, so we choose $\frac{\alpha_{y}, 0}{\beta_{y}, 0} \simeq 2.0$. Then, we restrict that even further, choosing $\alpha_{y, 0}<10 \mathrm{~m}$, such that the beam remains small in the vertical dimension. We want $\beta_{x, 0}$ at the entrance of EEX2 to be "large", which increases the final energy spread while reducing the final bunch length, which assists in making 
the final microbunches well-defined as well as increasing the overall compression ratio.

We test the 2XEEX configuration using a train of microbunches having the properties shown in Table 6.7 .

Table 6.7: Initial beam parameters for the test microbunch train used for the Double EEX simulations.

\begin{tabular}{ccc}
\hline Parameter & Value & Units \\
\hline \hline$\varepsilon_{x, 0}$ & 1.0 & $\mu \mathrm{m}$ \\
$\varepsilon_{y, 0}$ & 0.1 & $\mu \mathrm{m}$ \\
$\sigma_{z, 0}$ & 10.0 & $\mu \mathrm{m}$ \\
Energy Chirp & 1.0 & $\mathrm{~m}^{-1}$ \\
$\sigma_{\delta, 0}$ & $0.02 \mathrm{e}-4$ & - \\
Beamlet Separation & 30.0 & $\mu \mathrm{m}$ \\
Number of Beamlets & 9.0 & - \\
\hline
\end{tabular}

These beam parameters do not match to specific configurations from the ASTA injector, and are used only to demonstrate the possible compression and influence of second-order effects.

The matching conditions are selected for the center-most beamlet. Then the same quadrupole magnet settings are used for the entire bunch train, as the priority is to achieve compression while ending up with the individual beamlets having upright final LPS. In Figure 6.16 the simplified design is shown, with and without second-order effects.

In the absence of second-order effects, there is over a 10x compression factor, while also making the final beamlets have a more distinct longitudinal compression by fitting for Eqs. 6.8 at the entrance of the second emittance exchanger. These benefits come at the expense of the increase energy spread, both for individual beamlets and of the total bunch train, which now has a net energy chirp. Second-order ef- 

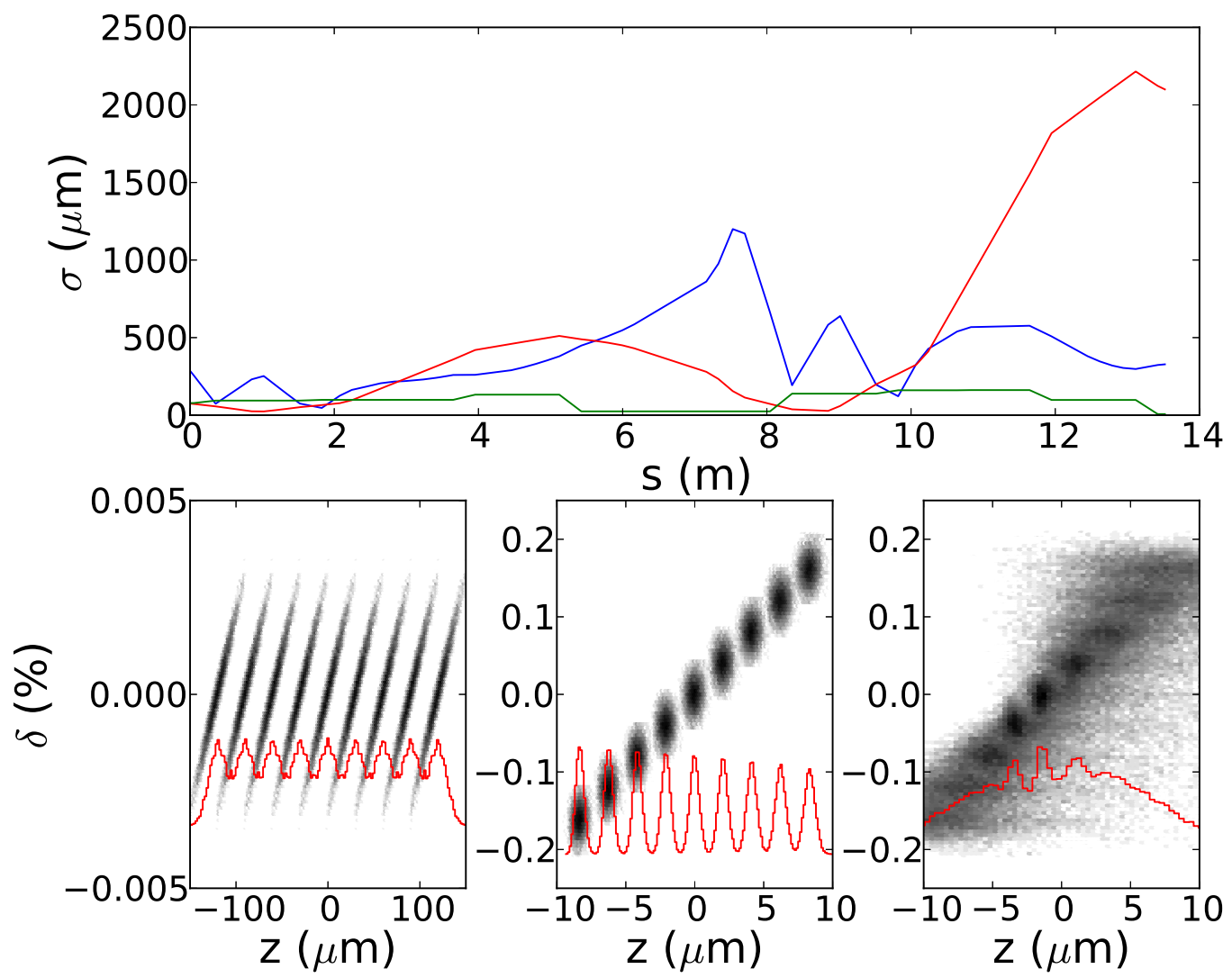

Figure 6.16: Double emittance exchanger beamline (Top) with horizontal (blue), vertical (red) and longitudinal (green) RMS beam sizes, with schematic overlay. LPS and projection (red) before first EEX (Lower Left) and after second EEX without second order effects (Lower Middle) and with second order effects (Lower Right), using only first-order transfer matrices and no SC or CSR. The telescope covers the range of $s=[5.5,8] \mathrm{m}$

fects significantly blur all but the central-most beamlets, which demonstrates the importance of controlling the transverse beam size, which is significantly increased due to both the transverse shear of the TDC as well as due to optics within the middle telescope. The center-most beamlet remains partially compressed, as the second-order effects it experiences are less than the beamlets further off-axis. 


\subsection{Conclusion}

A transverse-to-longitudinal emittance exchanger utilizing a variable- $R_{56}$ chicane has been found to function comparably well to the experimentally demonstrated double-dogleg design for the purposes of emittance exchange and its desired function as a bunch shaper. Advanced emittance-exchange concepts such as dispersionboosting were shown to be viable even when including realistic beamline dynamics. Collective effects including transverse space-charge, longitudinal space-charge, and longitudinal coherent synchrotron radiation have been shown to degrade the quality of emittance exchange as well as the ability to transmit and shape finely-structured beams. Proper control of beam dynamics through any emittance exchanger design is absolutely vital to the mitigation of both second-order effects as well as collective effects. Dispersion-boosting comes at the expense of shaping due to increased second-order effects.

Beam and operational parameters for emittance exchangers of any design must be judiciously selected, with the range of viable parameters decreasing as the target performance of the EEX becomes more stringent. Shaping, dispersion-boosting, and the numeric quality of the emittance come at trade-offs with each other due to mutually exclusive quadrupole magnet settings, second-order effects, and collective effects. 


\section{CHAPTER 7 CONCLUSION}

Experimental requirements demand demand the development of new acceleration and beam manipulation techniques which necessitates the completion of detailed simulation studies. The studies presented in this dissertation will contribute to the Advanced Superconducting Test Accelerator's position as a leading facility for such AAR\&D.

The need for high bunch currents requires bunch compression, but the realities of the ASTA facility require the compressor to be placed in the injector, where collective effects are a significant detriment to beam emittance. The detailed study performed for this dissertation explored in detail the tradeoffs between bunch charge, bunch length, compression, peak currents, and beam brightnesses for the purpose of presenting the full range of beam parameters that can be achieved by the facility. This study helped present to collaborators and future users what type of beam can be achieved for beam experiments. In particular, these studies revealed that lower bunch charges (i.e., those below the nominal $3.2 \mathrm{nC}$ goal of the ASTA facility) may

offer significant benefits over high bunch charges, due to the lower emittances and thus higher brightnesses that these allow.

The diagnostic capabilities of particle accelerators place limits on their experimental capabilities for several key reasons. An inability to measure low-charge beams due to thresholds on techniques, or coarse detection methods that cannot discern low emittance beams, may make it impossible to detect the beams before or after an experimental system. By completing a study of the proposed diagnos- 
tic system, I was able to demonstrate the range of beam parameters that can be succesfully be explored in the injector.

Transverse-to-Longitudinal emittance exchange will serve as a fundamental future for several future particle accelerators, and the design I completed for an in-line, dispersion-boosted exchanger demonstrated the system's technical feasibility for use in the ASTA beamline. Both the quantitative exchange of emittances and the more qualitative shaping translation open up new possibilities for beam-driven experiments such as dielectric wakefield acceleration, which is of particular interest both to our group at ASTA, as well as those at other national laboratories, namely the LANL and ANL groups that we have worked closely with. The studies, for the first time, demonstrated with simulations many of the key difficulties contained in controlling the transverse dynamics for dispersion-boosted configurations, or configurations that contain multiple emittance exchangers.

The start-to-end simulation tools and related analysis scripts that formed the backbone of the countless simulations performed for this dissertation will serve the ASTA group for years to come. In particular, my development work on GLUETRACK will enable my successors to more easily perform further detailed studies and modeling of the full ASTA facility. The barrier between the plethora of necessary codes, from their formats to broader issues regarding user friendliness, has served as a signifcant hindrance to the field of simulation accelerator physics, and it is my hope that my work has lowered the barriers to performing full start-to-end simulations. 


\section{REFERENCES}

[1] Walter Henning, Charles Shank, et al. Accelerators for America's future. Technical report, U.S. Department of Energy, 2012.

[2] CERN Communication Group. LHC: The guide. CERN-Brochure-2009-003Eng, 2009.

[3] R.R. Wilson. The Tevatron. FERMILAB-TM, 0763, 1978.

[4] Foote et al. The clinical case for proton beam therapy. Radiation Oncology 2012, 7:174, 2012.

[5] S.B. Felch, M.I. Current, and M.C. Taylor. Ion implantation for semiconductor devices: The largest use of industrial accelerators. Proceedings of the North American Particle Accelerator Conference 2013, page 740, 2013.

[6] F. Lemery, D. Mihalcea, and P. Piot. Experimental plans to explore dielectric wakefield acceleration in the $\mathrm{THz}$ regime. Proceedings of the 2011 International Particle Accelerator Conference, page 2781, 2011.

[7] M. Rosing and W. Gai. Longitudinal- and transverse-wake-field effects in dielectric structures. Phys. Rev. D, 42:1829, 1990.

[8] M. Cornacchia and P. Emma. Transverse to longitudinal emittance exchange. SLAC-PUB, 9225, 2002.

[9] J.D. Jackson. Classical Electrodynamics, Third Edition. Wiley, 1998.

[10] K.-J. Kim. Three-dimensional analysis of coherent amplification and selfamplified spontaneous emission in free-electron lasers. Physical Review Letters, $57: 18711874,1986$.

[11] C. Lejeune and J. Aubert. Emittance and brightness: Definitions and measurements. Advances in Electornics and Electron Physics Supplement, 13A:159-259, 1980 .

[12] K. Kubo. How to calculate intrinsic emittances from 4-dimension beam-matrix. KEK Report 99-02, 1999.

[13] R. Brinkmann, Y. Derbenev, , and K. Flöttmann. A low emittance, flat-beam electron source for linear colliders. Physical Review Special Topics - Accelerators and Beams, 4:053501, 2001. 
[14] K.-J. Kim. Round-to-flat transformation of angular-momentum-dominated beams. Physical Review Special Topics - Accelerators and Beams, 6:104002, 2003.

[15] P. Piot, Y.-E. Sun, and K.-J. Kim. Photoinjector-generation of a flat electron beam with transverse emittance ratio of 100. Physical Review Special Topics Accelerators and Beams, 9, 2006.

[16] A. Chao. Physics of Collective Beam Instabilities in High Energy Accelerators. Wiley \& Sons, Inc., 1993.

[17] E.L. Saldin et al. On the coherent radiation of an electron bunch moving in an arc of a circle. Nuclear Instruments and Methods in Physics Research, A 398:373-394, 1997.

[18] M. Borland. Simple method for particle tracking with coherent synchrotron radiation. Physical Review Special Topics - Accelerators and Beams, 4:070701, 2001.

[19] M. Church et al. Proceedings of the Particle Accelerator Conference 200\%, page 2942.

[20] B. Dwersteg, K. Flöttmann, J. Sekutowicz, and Ch. Stolzenburg. RF gun design for the TESLA vuv free electron laser. Nuclear Instruments and Methods in Physics Research A, 393:93-95, 1997.

[21] P. Piot, C. R. Prokop, B.E. Carlsten, D. Mihalcea, and Y.-E. Sun. Flat electron bunch compression at the advanced superconducting test accelerator. Proceedings of International Particle Accelerator Conference 2013, pages 2003-2005, 2013.

[22] T. Smith. Intense low emittance linac beams for free electron lasers. Proceedings of the 1984 Linear Accelerator Conference, SLAC report 303:411, 1986.

[23] D. H. Dowell, T. D. Hayward, and A. M. Vetter. Magnetic pulse compression using a third harmonic rf linearizer. Proceedings of the 1995 Particle Acceleration Conference, pages 992-994, 1996.

[24] K. Flöttmann, T. Limberg, and P. Piot. Generation of ultrashort electron bunches by cancellation of nonlinear distortions in the longitudinal phase space. DESY Report, TESLA-FEL 2001-06, 2001.

[25] M. Rihaoui, D. Mihalcea, W. Gai, P. Piot, and J. G. Power. Design and analysis of a single-shot longitudinal phase space measurement at the Argonne Wakefield Accelerator. Proceedings of the 2010 Advanced Accelerator Concept (AAC10) workshop, pages 16-19, 2010. 
[26] C.R. Prokop et al. High-energy lattice for first-beam operation of the SRF test accelerator at NML. FERMILAB-TM-2516-APC, 2011.

[27] D. Schulte. Main linac basics. 7th Linear Collider School, 2010.

[28] J. B. Rosenzweig and L. Serafini. Transverse particle motion in radio-frequency linear accelerators. Physical Review E, 49:1599-1602, 1994.

[29] P. Tenenbaum. Emittance measurements in CTF2 drive beam. CLIC-Note, $326,1997$.

[30] A.H. Lumpkin. OTR imaging considerations for ILC-TA at NML. Fermilab Note, pages Beams-doc-2930-v1, 2007.

[31] A.H. Lumpkin et al. Initial beam-profiling tests with the NML prototype station at the Fermilab A0 photoinjector. Proceedings of Particle Accelerator Conference 2011, page MOP219, 2011.

[32] P. Emma M. Woodley. Measurement and correction of cross-plane coupling in the transport lines. LINAC 2000, pages 196-198, 2000.

[33] MATLAB. MATLAB: 2010. The MathWorks Inc., 2010.

[34] C.R. Prokop et al. Longitudinal Phase Space Measurement for the Advanced Superconducting Test Accelerator photoinjector. Proceedings of the International Particle Accelerator Conference 2012, page 3009, 2012.

[35] M. Borland. elegant: A flexible SDDS-compliant code for accelerator simulation,. Advanced Photon Source, LS-287, 2000.

[36] M. Dohlus and T. Limberg. Impact of optics on CSR-related emittance growth in bunch compressor chicanes. Proceedings of Particle Accelerator Conference 2005, pages 1015-1017, 2005.

[37] D. H. Dowell, S. Joly, A. Loulergue, et al. Space charge limits of longitudinal emittance in RF photoinjectors. AIP Conf Proc., 398:793, 1997.

[38] B.J.Claessens et al. Ultracold electron sources. Physical Review Letters, 95:164801, 2005.

[39] F. Stulle, A. Adelmann, and M. Pedrozzi. Designing a bunch compressor chicane for a multi-TeV linear collider. Physical Review Special Topics - Accelerators and Beams, 10:031001, 2007.

[40] S. Di Mitri et al. Transverse emittance preservation during bunch compression in the Fermi free electron laser. Physical Review Special Topics - Accelerators and Beams, 15:020701, 2012. 
[41] M. Dohlus and T. Limberg. Impact of optics on CSR-related emittance growth in bunch compressor chicanes. Proceedings of the 2005 Particle Accelerator Conference, page 1015, 2005.

[42] C.R. Prokop et al. Simulation studies of the low-energy bunch compressor at the Advanced Superconducting Test Accelerator. FNAL-TM-2533-APC, 2012.

[43] K. L. F. Bane, P. Chen, and P. B. Wilson. On collinear wakefield acceleration. SLAC-PUB-3662, 1985.

[44] P. Piot, C. Behrens, C. Gerth, M. Dohlus, F. Lemery, D. Mihalcea, P. Stoltz, and M. Vogt. Generation and characterization of electron bunches with ramped current profiles in a dual-frequency superconducting linear accelerator. Physical Review Letters, 108:034801, 2012.

[45] P. Chen, J.M. Dawson, Robert W. Huff, and T. Katsouleas. Acceleration of electrons by the interaction of a bunched electron beam with a plasma. Physical Review Letters, 54:693, 1985.

[46] J. S. Nodvick and D. S. Saxon. Suppression of coherent radiation by electrons in a synchrotron. Physical Review, 96:180, 1954.

[47] A. Potylitsyn, M. I. Ryazanov, M. N. Strikhanov, and A. A. Tishchenko. Diffraction radiation from relativistic particles. ISBN 978-3-642-12513-3, 239, 2011.

[48] A.H. Lumpkin et al. Synchroscan streak camera imaging at a $15-\mathrm{MeV}$ photoinjector with emittance exchange. Nuclear Instrumentation and Methods, A687:92-100, 2012.

[49] C. Prokop, P. Piot, M. C. Lin, , and P. Stoltz. Numerical modeling of a table-top tunable Smith-Purcell free-electron laser operating in the super-radiant regime. Appl. Phys. Lett., 96:151502, 2010.

[50] D. Mihalcea, P. Piot, , and P. Stoltz. Three-dimensional analysis of wakefields generated by flat electron beams in planar dielectric-loaded structures. Physical Review Special Topics - Accelerators and Beams, 15:081304, 2012.

[51] C. R. Prokop, P. Piot, B.E. Carlsten, and M.D. Church. Performance of lowenergy magnetic bunch compression for the ASTA photoinjector at Fermilab. Proceedings of International Particle Accelerator Conference 2012, pages 30062008, 2012.

[52] D. Edwards. Notes on transit of particles in deflecting mode pillbox cavity. Fermilab Technical Memo, 2007. 
[53] A. Gover. Superradiant and stimulated-superradiant emission in prebunched electron-beam radiators. i. formulation. Physical Review Special Topics - Accelerators and Beams, 8:030701, 2005.

[54] P. Schütt, T. Weiland, and V. M. Tsakanov. On the wake field acceleration using a sequence of driving bunches. Proceedings of the Second All-Union Conference on New Methods of Charged Particle Acceleration, pages 12-17, 1989.

[55] J. B. Rosenzweig, D. B. Cline, B. Cole, H. Figueroa, W. Gai, R. Konecny, J. Norem, P. Schoessow, and J. Simpson. Experimental observation of plasma wake-field acceleration. Physical Review Letter, 61:98, 1988.

[56] M. C. Thompson et al. Breakdown limits on gigavolt-per-meter electron-beamdriven wakefields in dielectric structures. Physical Review Letters, 100:214801, 2008.

[57] W.B. Mori. Talk given at the Advanced Accelerator Concept workshop (AAC08), 2008.

[58] P. Piot, D.R. Douglas, and G.A. Krafft. Longitudinal phase space manipulation in energy recovering linac-driven free-electron lasers. Physical Review Special Topics - Accelerators and Beams, 6:030702, 2004.

[59] R. J. England, J. B. Rosenzweig, , and G. Travish. Generation and measurement of relativistic electron bunches characterized by a linearly ramped current profile. Physical Review Letters, 100:214802, 2008.

[60] M. Bolosco, I. Boscolo, F. Castelli, S. Cialdi, M. Ferrario, V. Petrillo, and C. Vaccarezza. Generation of short $\mathrm{THz}$ bunch trains in a $\mathrm{RF}$ photoinjector. Nuclear Instrumentation and Methods, A 577:409, 2007.

[61] Y.-C. Huang. Laser beat-wave bunched beam for comapct supperradiance sources. International Journal of Modern Physics, B 21:287, 2007.

[62] Y. Li and K.-J Kim. Nonrelativistic electron bunch train for coherently enhanced terahertz radiation sources. Applied Physics Letters, 92:014101, 2008.

[63] D. C. Nguyen and B. E. Carlsten. Amplified coherent emission from electron beams prebunched in a masked chicane. Nuclear Instrumentation and Methods, A 375:597, 1996.

[64] P. Muggli, V. Yakimenko, M. Babzien, E. Kallos, and K. P. Kusche. Generation of trains of electron microbunches with adjustable sub-picosecond spacing. Physical Review Letters, 101:054801, 2008. 
[65] Y. Orlov, C. M. O’Neill, J. J. Welch, , and R. Sieman. B factory optics and beam-beam interaction for millimeter $\beta^{*}$ and locally shortened bunches. Proceedings of the 1991 Particle Accelerator Conference, page 2838, 1991.

[66] M. Cornacchia, , and P. Emma. Longitudinal phase space manipulation in energy recovering linac-driven free-electron lasers. Physical Review Special Topics - Accelerators and Beams, 6:030702, 2003.

[67] P. Emma, Z. Huang, K.-J. Kim, , and P. Piot. Transverse-to-longitudinal emittance exchange to improve performance of high-gain FELs. Physical Review Special Topics - Accelerators and Beams, 9:100702, 2006.

[68] R. Fliller. General solution for a transverse-to-longitudinal emittance exchange beamline and cavity gradient sensitivity. Fermilab Report, BeamDocs 2271-v2, 2007.

[69] P. Piot. Alternative lattice options for energy recovery in high-average-power high-efficiency free-electron lasers. Proceedings of the 2nd workshop on high power high average power electron beams, FERMILAB-CONF-09-057-APC, 2009.

[70] D. Xiang and A. Chao. Emittance and phase space exchange for advanced beam manipulation and diagnostics. Physical Review Special Topics - Accelerators and Beams, 14:114001, 2011.

[71] J. Ruan et al. First observation of the exchange of transverse and longitudinal emittances. Physical Review Letters, 106:244801, 2011.

[72] Y.-E. Sun. Tunable subpicosecond electron-bunch-train generation using a transverse-to-longitudinal phase-space exchange technique. Physical Review Letters, 105:234801, 2010.

[73] P. Piot, Y.-E. Sun, J.G. Power, and M. Rihaoui. Generation of relativistic electron bunches with arbitrary current distribution via transverse-to-longitudinal phase space exchange. Physical Review Special Topics - Accelerators and Beams, 14:022801, 2011.

[74] A.A. Zholents and M.S. Zolotorev. A new type of bunch compressor and seeding of a short wave length coherent radiation. ANL/APS/LS-32\%, 2011.

[75] Ji Qiang et al. An object-oriented parallel particle-in-cell code for beam dynamics simulation in linear accelerators. Journal of Computational Physics, 163:434, 2000.

[76] M.D. Dohlus et al. CSRtrack: Faster calculation of 3-D CSR effects. Proceedings of the 2004 FEL Conference, pages 18-21, 2004. 
[77] C.R. Prokop et al. Beam dynamics performances and applications of a lowenergy electron-beam magnetic bunch compressor. Nuclear Instruments and Methods in Physics Research A, 719:17-28, 2013.

[78] K.L. Brown. A first- and second-order matrix theory for the design of beam transport systems and charged particle spectrometers. SLAC Report, 75:69$136,1982$.

[79] E. Branlard. Study of a 5 cell copper cavity at $3.9 \mathrm{GHz}$. Fermilab Technical Memo, 2007.

[80] M. Borland. User's manual for ELEGAnt. 2011.

[81] Ya. S. Derbenev and V.D. Shiltsev. Transverse effects of microbunch radiative interaction. SLAC-PUB-־181, 1996.

[82] Klaus Flöttmann. ASTRA: A Space Charge Tracking Algorithm, version 3.0. 2011.

[83] M. Dohlus. Two methods for the calculation of CSR fields. DESY Report, TESLA-FEL-2003-05, 2003.

[84] I. Zagorodnov. GlueTrack interpreter for S2E beam dynamic simulations. Private communication, 2011. 
APPENDIX A

TRANSFER MATRICES OF BEAMLINE ELEMENTS 
The purpose of this appendix is to present more detail on the use of transfer matrices that was discussed in Chapter 2, as well as to present the transfer matrices of many common beamline components, such as drifts, dipoles, and quadrupoles, as well as the transfer matrices of several compound elements that contain several components, such as bunch compressors and emittance exchangers.

\section{A.1 Basics of Transfer Matrices}

Many beamline elements can be reasonably-well described with a first-order transfer matrix that represent the relationship between a particle's final and initial coordinates, relative to the reference particle that was described in Chapter 2. We use the notation where $M$ represents a transfer matrix, $R_{i j}$ represents a specific element, with $i$ and $j$ corresponding to its respective row and element, respectively.

As a simplification for the sake of demonstration, let us assume that motion in the three directions is uncoupled (as is the case for focusing and defocusing quadrupoles). In that case, the final $x$ and $x^{\prime}$ depend only on the transfer matrix of the element, and on the initial parameters $x_{0}$ and $x_{0}^{\prime}$.

$$
\left[\begin{array}{l}
x \\
x^{\prime}
\end{array}\right]=\left[\begin{array}{ll}
A & B \\
C & D
\end{array}\right]\left[\begin{array}{l}
x_{0} \\
x_{0}^{\prime}
\end{array}\right]
$$

where A, B, C, and D are the elements of the 2-D transfer map. This matrix represents the set of equations

$$
\begin{gathered}
x=A x_{0}+B x_{0}{ }^{\prime} \\
x^{\prime}=C x_{0}+D x_{0}{ }^{\prime} .
\end{gathered}
$$


Likewise, the fully six-dimensional transfer matrix can be represented with the same formalism.

$$
\left[\begin{array}{l}
x \\
x^{\prime} \\
y \\
y^{\prime} \\
z \\
\delta
\end{array}\right]=\left[\begin{array}{cccccc}
R_{11} & R_{12} & R_{13} & R_{14} & R_{15} & R_{16} \\
R_{21} & R_{22} & R_{23} & R_{24} & R_{25} & R_{26} \\
R_{31} & R_{32} & R_{33} & R_{34} & R_{35} & R_{36} \\
R_{41} & R_{42} & R_{43} & R_{44} & R_{45} & R_{46} \\
R_{51} & R_{52} & R_{53} & R_{54} & R_{55} & R_{56} \\
R_{61} & R_{62} & R_{63} & R_{64} & R_{65} & R_{66}
\end{array}\right]\left[\begin{array}{c}
x_{0} \\
x_{0}^{\prime} \\
y_{0} \\
y_{0}^{\prime} \\
z_{0} \\
\delta_{0}
\end{array}\right] .
$$

The rules of matrix multiplication apply. For example, a dipole bend, followed a drift, followed by another dipole bend, would have a total transfer matrix

$$
M_{\text {Total }}=M_{\text {Dipole }} M_{\text {Drift }} M_{\text {Dipole }}
$$

With this methodology in place, an entire beamline can be calculated by multiplying the transfer elements in the proper order, resulting in the transfer matrix of the complete beamline. This is a powerful tool, and decent as long as beamline components may be accurately modeled by only their first-order effects. We will see in the next section the caveats involved with this formulation, notably higher-order effects and collective effects. 


\section{A.2 Exceptions to Linear Transfer Maps}

The transfer matrices discussed in the previous section are only the first-order transfer matrices, and only account for linear relationships between final initial coordinates. This is not always the case. There are often times higher-order relationships, or relationships that cannot be described with transfer matrices. For example, a kick to $x^{\prime}$ may depend on $x_{0}^{2}$, or the product of $x y$. We represent the elements of the second-order transfer matrices as $T_{i j k}$, where the $k$ is the other dependence. Second-order matrices have over $6^{3}=216$, though the array is, fortunately, symmetric, i.e. $T 113=T 131$ due to the commutative property, $x y=y x$.

Due to the exponential nature of this matrix (dependence on $x^{2}, y^{2}, x x^{\prime}$,, etc.), second-order effects generally become more prominent for "large" beams. This is not just spatially large, but also beams with large energy spreads $(\delta)$, or correlations such as being large radially, i.e. the product of $x$ and $y$.

Collective effects, i.e. inter-particle or inter-bunch interactions, are highly nonlinear. For example, the longitudinal space charge in Equation 2.22 or the coherent synchrotron radiation in Equation 2.24 are not linear functions of $z$, nor are they clearly second-order or higher order. Therefore, there is no transfer matrix that can be used to represent their effects. This makes them very difficult to model, which in turn requires elaborate simulations to model correctly.

In this dissertation, we may discuss specific second-order terms, but primarily use their presence as justification to keep the beam small, as beam emittances are not conserved under second-order transformations. 


\section{A.3 Transfer Matrices of Common Beamline Components}

In this section, I will detail the transfer matrices of many common beamline components. The simplest transfer matrices are those of simple drifts, in which the slopes $x^{\prime}$ and $y^{\prime}$ remain constant and there is no change to the longitudinal parameters, $z$ or $\delta$. As $x^{\prime}=\frac{\partial x}{\partial s}$ and $y^{\prime}=\frac{\partial y}{\partial s}, x=x_{0}+L x^{\prime}$, and similarly for $y$, where $L$ is the drift length. The transfer matrix is

$$
M_{\text {Drift }}=\left[\begin{array}{llllll}
1 & 0 & 0 & 0 & 0 & 0 \\
L & 1 & 0 & 0 & 0 & 0 \\
0 & 0 & 1 & 0 & 0 & 0 \\
0 & 0 & L & 1 & 0 & 0 \\
0 & 0 & 0 & 0 & 1 & 0 \\
0 & 0 & 0 & 0 & 0 & 1
\end{array}\right]
$$

While a drift is the simplest beamline element with most of the terms being 0 . In fact, ANY beamline element that doesn't couple two dimensions is block-diagonal, i.e. only the diagonal $2 \times 2$ blocks are filled. As we want beamline components that preserve emittances, most of the transfer matrices that we will deal have 0 in most of their terms. Therefore, we will often stick to just the $2 \times 2$ transfer matrices of the relevant transformation, or $4 \times 4$ matrices when two dimensions are coupled or acted upon. One such example of the latter is quadrupole magnets, which focus in $x$ and defocus in $y$, or vice-versa. 
In the thick-lens approximation, quadrupoles are instantaneous kicks to the momentum that are linear with the distance from the center of the beamline. This leads to a transfer in the focusing $(+)$ and defocusing $(-) 2 \times 2$ blocks of, respectively,

$$
M_{Q, \pm}=\left[\begin{array}{cc}
1 & 0 \\
- \pm k & 1
\end{array}\right]
$$

There is no change to the longitudinal elements, and no coupling between any two dimensions unless the quadrupole is rotated, which is known as a skew quadrupoles.

Dipole magnets are another common beamline element. This is actually somewhat complicated, as the orientation of the dipole faces influences the transverse focusing. First we consider as "sector dipole", for which the beam enters and exits the magnetic field perpendicular. The bending radius is dependent on the particle energy. Particles with a higher energy than the reference particle bend less, which results in a change to $x^{\prime}$ and $x$. Likewise, those with lower energies bend more. Here we consider a sector dipole in the $x-z$ plane, which has a transfer matrix of

$$
M_{\text {SectorDipole }}=\left[\begin{array}{cccccc}
\cos (\theta) & L & 0 & 0 & 0 & R[1-\cos (\theta)] \\
-\frac{\sin (\theta)}{R} & \cos (\theta) & 0 & 0 & 0 & \sin (\theta) \\
0 & 0 & 1 & L & 0 & 0 \\
0 & 0 & 0 & 1 & 0 & 0 \\
-\sin (\theta) & -R[1-\cos (\theta)] & 0 & 0 & 1 & -R[\theta-\sin (\theta)] \\
0 & 0 & 0 & 0 & 0 & 1
\end{array}\right]
$$

It is important to note that sector dipoles have no focusing or defocusing effect in $y$, to first order. Real dipoles are not necessarily sector dipoles. For example, with a rectangular dipole, one of the faces is that of a sector dipole while the other is at 
some angle, or both faces of the magnetic field are at some angle. These corrections also add correlations for the $y$ dynamics. This introduces a counter-intuitive effect. The effective length of the magnetic field now depends on $x$. This can be corrected for with a pair of matrices for the edges. These are represented as [78]

$$
M_{E d g e}=\left[\begin{array}{cccccc}
1 & 0 & 0 & 0 & 0 & 0 \\
\frac{\tan (\delta)}{R} & 1 & 0 & 0 & 0 & 0 \\
0 & 0 & 1 & 0 & 0 & 0 \\
0 & 0 & F & 1 & 0 & 0 \\
0 & 0 & 0 & 0 & 1 & 0 \\
0 & 0 & 0 & 0 & 0 & 1
\end{array}\right]
$$

where $\delta$ is the angular deviation of the dipole's face relative to perpendicular to the beam's trajectory, and

$$
\begin{array}{r}
F \equiv-\frac{1}{R} \tan \delta-\psi \\
\psi \equiv g K[\sec \delta]\left[1+\sin \delta^{2}\right]
\end{array}
$$

where $g$ is the gap between the faces of the dipole and $K$ is a parameter related to the strength of the edge-effect of the fields.

Transverse deflecting cavities (TDCs) are of significant interest for various diagnostic and shaping processes (see Chapters 5 and 6 for more details). Following the work detailed in Reference [52] [79], the 4x4 transfer matrix (neglecting $y$ and $y^{\prime}$ for compactness) of a single-cell TDC operated at $\phi=0$ is derived and presented in Appendix B. In an actual experiment, a transverse deflecting cavity is comprised of several cells, so the effect is cumulative over several cells of finite-thickness. 


$$
M_{N-C e l l}=M_{1-C e l l}^{N}
$$

For a five-cell cavity, such as that used at A0, we find

$$
\left[\begin{array}{cccccc}
1 & 5 \frac{\lambda}{2} & 0 & 0 & 25 \kappa \lambda & 0 \\
0 & 1 & 0 & 0 & 5 \kappa & 0 \\
0 & 0 & 1 & L & 0 & 0 \\
0 & 0 & 0 & 1 & 0 & 0 \\
0 & 0 & 0 & 0 & 1 & 0 \\
5 \kappa & 25 \kappa \frac{\lambda}{4} & 0 & 0 & 85 \kappa^{2} \frac{\lambda}{8} & 1
\end{array}\right]
$$

noting that we switched back the $\lambda$ notation so it is clear which length we are using. This comparison is not entirely fair, as we aren't going to be using five times the power. If we use $\kappa \rightarrow \frac{\kappa}{5}$, the matrix should then be

$$
\left[\begin{array}{cccccc}
1 & 5 \frac{\lambda}{2} & 0 & 0 & \kappa \lambda \frac{5}{4} & 0 \\
0 & 1 & 0 & 0 & \kappa & 0 \\
0 & 0 & 1 & L & 0 & 0 \\
0 & 0 & 0 & 1 & 0 & 0 \\
0 & 0 & 0 & 0 & 1 & 0 \\
\kappa & \lambda \kappa \frac{5}{4} & 0 & 0 & \lambda \kappa^{2} \frac{17}{40} & 1
\end{array}\right] .
$$

The notable effect of using multiple matrices is that it reduces the magnitude of the $R_{65}$ term, relative to the terms that we are interested in making use of which are typically the $R_{25}$ and $R_{61}$ terms, i.e. the transverse kick as a function of $z$ and the energy kick as a function of $x$. However, the $R_{65}$ term is still present, and for further correction we can use an accelerating mode cavity adjacent to the TDC. 
Operated at zero-crossing where there is zero acceleration for the reference particle, the transfer matrix is:

$$
M_{A c c}=\left[\begin{array}{cccccc}
1 & L & 0 & 0 & 0 & 0 \\
0 & 1 & 0 & 0 & 0 & 0 \\
0 & 0 & 1 & L & 0 & 0 \\
0 & 0 & 0 & 1 & 0 & 0 \\
0 & 0 & 0 & 0 & 1 & 0 \\
0 & 0 & 0 & 0 & \frac{k V}{E} & 1
\end{array}\right]
$$

where $A$ is some parameter related to the cavity strength. If set correctly to cancel out $M_{T D C}$ 's $R_{65}$,

$$
M_{A c c} M_{T D C}=\left[\begin{array}{cccccc}
1 & 5 \frac{\lambda}{2} & 0 & 0 & \kappa \lambda \frac{5}{4} & 0 \\
0 & 1 & 0 & 0 & \kappa & 0 \\
0 & 0 & 1 & L & 0 & 0 \\
0 & 0 & 0 & 1 & 0 & 0 \\
0 & 0 & 0 & 0 & 1 & 0 \\
\kappa & \lambda \kappa \frac{5}{4} & 0 & 0 & 0 & 1
\end{array}\right] .
$$

The true power of this design will be shown when emittance exchanger designs and transfer matrices are presented.

\section{A.4 Emittance Exchangers and Block Anti-Diagonality}

Any emittance exchanger (EEX) that we study is comprised of two doglegs and a deflecting-mode cavity at its center. Since the exchange is between the horizontal 
and transverse phase spaces, we work with $4 \mathrm{x} 4 \mathrm{x}-\mathrm{z}$ transfer matrices. First, we look at a design with two identical doglegs, with transfer matrices $M_{-}$and $M_{+}$, which are the doglegs upstream and downstream of the TDC, respectively.

$$
\begin{aligned}
M_{-}= & {\left[\begin{array}{cccc}
1 & L_{-} & 0 & \eta_{-} \\
0 & 1 & 0 & 0 \\
1 & \eta_{-} & 0 & R_{56-} \\
0 & 0 & 0 & 1
\end{array}\right] } \\
M_{+}= & {\left[\begin{array}{cccc}
1 & L_{+} & 0 & \eta_{+} \\
0 & 1 & 0 & 0 \\
1 & \eta_{+} & 0 & R_{56+} \\
0 & 0 & 0 & 1
\end{array}\right] }
\end{aligned}
$$

The simplest EEX would be one using the two identical doglegs $\left(\eta_{-}=\eta_{+}\right.$with identical bend angles for the 1st and 2nd dipoles, respectively), using the thick-lens model. For the chicane EEX and boosted-dispersion EEX, we use modified doglegs with quadrupoles, which can tune several terms of dogleg transfer matrix, most notably the dispersion on $R_{56}$, which is associated with its compression. With a thin-lens model, there is no residual coupling term for the final EEX's first-order transfer matrix. When the basic requirements are met, the transfer matrix has the form

$$
M_{E E X}=\left(\begin{array}{cccc}
0 & 0 & R_{15} & R_{16} \\
0 & 0 & R_{25} & R_{26} \\
R_{51} & R_{52} & 0 & 0 \\
R_{61} & R_{62} & 0 & 0
\end{array}\right) \mathbf{X}_{\mathbf{0}}
$$


From this, we can conclude that the $R_{65}$ term of the TDC transfer matrix leads to residual coupling between the exchanged planes, which ruins the quality of the emittance exchange. 


\section{APPENDIX B}

DERIVATIONS OF THE TRANSFER MATRIX AND DEFLECTING KICK OF A TRANSVERSE DEFLECTING CAVITY 
In this appendix, I explore a detailed derivation of the deflecting strength and transfer matrix of a transverse deflecting cavity.

A key factor in the performance of a longitudinal spectrometer is the effect of the transverse deflecting cavity. For the purpose of mapping the longitudinal profile in to the vertical plane, only the transverse kick, i.e. the $R_{15}$ term, is desired. However, the transfer matrix of a TDC contains other terms, which produce terms that are detrimental to both one's use in a spectrometer or an emittance exchanger, which is discussed in Chapter 6. Here, we explore a derivation of the transverse deflecting cavity's transfer matrix of a pillbox cavity that approximates the TDC that will be used at ASTA, as well as the relationship between power and the deflecting kick. We use a cavity that extends for $-\frac{\lambda}{4} \geq z \geq \frac{\lambda}{4}$, such that the cavity can be operated at zero-crossing for the reference particle, i.e. that it receives no transverse kick.

We start with the known fields near the axis of a pillbox cavity operating in the $\mathrm{TM}_{110}$ mode.

$$
\begin{aligned}
& E_{s}(\vec{r}, t)=E_{0}(x) \cos (\omega t)=E^{\prime} x \cos (\omega t) \\
& B_{y}(\vec{r}, t)=B_{0}(x) \sin (\omega t)=\frac{E^{\prime}}{\omega} \cos (\omega t)
\end{aligned}
$$

where $E_{s}$ is the longitudinal electric field, $B_{y}$ is the vertical magnetic field, and $E_{0}(x)$ and $B_{0}(x)$ are the electric and magnetic fields as a function of distance from the central axis of the cavity. We can use a linear approximation for the two terms using $E^{\prime}=\partial E_{x} / \partial x$ at $t=0$ to find

$$
\begin{aligned}
& E_{s}(\vec{r}, t) \simeq E^{\prime} x \cos (\omega t) \\
& B_{y}(\vec{r}, t) \simeq \frac{E^{\prime}}{\omega} \cos (\omega t)
\end{aligned}
$$


Following the Lorentz force, $e(\vec{E}+\vec{v} \times \vec{B})$, we then have the relevant transverse and longitudinal forces on the reference particle (denoted by the subscript $r$ ),

$$
\begin{aligned}
& \left(\frac{d p_{x}}{d t}\right)_{r}=-\frac{e E^{\prime} v_{s, r}}{\omega} \sin (\omega t) \\
& \left(\frac{d p_{s}}{d t}\right)_{r}=-\frac{e E^{\prime} v_{x, r}}{\omega} \sin (\omega t)+e E^{\prime} x_{r} \cos (\omega t)
\end{aligned}
$$

However, we are concerned with arbitrary particles (denoted by the subscript i) that are not necessarily passing at zero-crossing, and see a field their distance $z_{i}$ from the center of the bunch. The fields seen by these particles are, expanding for $k z \ll 1$,

$$
\begin{aligned}
E_{s}(x, t)_{i} & =E^{\prime} x \cos \left(\omega t-k z_{i}\right) \simeq E^{\prime} x_{i} \cos (\omega t)+E^{\prime} k x_{i} z_{i} \sin (\omega t), \\
B_{y}(x, t)_{i} & =\frac{E^{\prime}}{\omega} \sin \left(\omega t-k z_{i}\right) \simeq \frac{E^{\prime}}{\omega} \sin (\omega t)+\frac{E^{\prime}}{c} z_{i} \cos (\omega t) .
\end{aligned}
$$

For the purpose of constructing the transfer matrix of the system, we must find the relative force between any particle and the reference particle. We also assume that the beam is highly relativistic, i.e.

$$
\begin{array}{r}
s \simeq c t \\
d s \simeq c d t \\
\frac{d p}{d t}=c \frac{d p}{d s}
\end{array}
$$

Substituting for the change of coordinates and noting that $\omega=k c$, we then have 


$$
\begin{aligned}
& \left(\frac{d p_{x}}{d s}\right)_{r}=-\frac{e E^{\prime}}{k c} \sin (k s), \\
& \left(\frac{d p_{s}}{d s}\right)_{r}=e E^{\prime} x_{r} \cos (k s)+\frac{e E^{\prime} v_{x, r}}{k c} \sin (k s), \\
& \left(\frac{d p_{x}}{d s}\right)_{i}=-\frac{e E^{\prime}}{k c} \sin (k s)+\frac{e E^{\prime}}{c} z \cos (k s), \\
& \left(\frac{d p_{s}}{d s}\right)_{i}=\frac{e E^{\prime}}{c} x_{i} \cos (k s)+\frac{e E^{\prime} v_{x, i}}{k c^{2}} \sin (k s) .
\end{aligned}
$$

For the purpose of developing a transfer matrix for the system, we are interested in the $d$ ifference between an arbitrary particle and the reference particle. This gives us the relative equations of motion

$$
\begin{aligned}
& \left(\frac{d p_{x}}{d s}\right)_{r e l}=\frac{e E^{\prime}}{c} z_{i} \cos (k s), \\
& \left(\frac{d p_{s}}{d s}\right)_{r e l}=e E^{\prime} x \cos (k s)+\frac{e E^{\prime}}{k c} \frac{d x}{d s} \sin (k s),
\end{aligned}
$$

using $x=x_{i}-x_{r}$, and $\frac{d x}{d s}=\frac{p_{x}}{p_{0}}$, where $p_{0}$ is the reference momentum at the entrance of the cavity. We also make use of

$$
\begin{aligned}
v_{x} & =\frac{d x}{d t}, \\
d t & =\frac{d s}{c}, \\
x & =x_{i}-x_{r}, \\
v_{x, i}-v_{x, r} & =\frac{d\left(x_{i}-x_{r}\right)}{d s}=\frac{d x}{d s} .
\end{aligned}
$$

This may change as the particle traverses the cavity, particularly in the case of operation at non-zero crossing. We also assume, for zero-crossing operation, that $\frac{d z}{d s}=0$. 
We can then integrate from $-\frac{\lambda}{4}$ to $\frac{\lambda}{4}$ to find the terms of the transfer matrix. When operating at zero-crossing and utilizing cavity with length of $\lambda / 2$, the integrals are fairly straightforward due to evaluations of sines and cosines at $0, \pi$, and $\pi / 2$.

At this point, most of the basic pieces have been completed. Next, we find

$$
p_{x}(s)=p_{0}+\left.\frac{e E^{\prime}}{k c} z[\sin (k s)]\right|_{-\lambda / 4} ^{s}=p_{x}\left(-\frac{\lambda}{4}\right)+\frac{e E^{\prime}}{k c} z[\sin (k s)+1],
$$

which we then must convert to $x^{\prime}$,

$$
x^{\prime} \equiv \frac{p_{x}}{p_{s}}=\frac{\gamma m v_{x}}{\gamma m v_{s}}=\frac{d x}{d s}
$$

We also define the cavity strength,

$$
\kappa \equiv \frac{2 e E^{\prime}}{c k p_{z}}
$$

Eq. B.22 becomes

$$
x^{\prime}(s) \sim x_{0}^{\prime}+\frac{\kappa z}{2}[\sin k s+1]
$$

evaluating at $s=\frac{\lambda}{4}$, and noting that $k=\frac{2 \pi}{\lambda}$,

$$
x_{f}^{\prime}=x_{0}^{\prime}+\frac{\kappa z}{2}[1+1]=x_{0}^{\prime}+\kappa z
$$

which is the row of the transfer matrix that applies the transverse kick linear in $z$ and $\kappa$, where the subscript 0 denotes the value at the entrance of the TDC. 
Integration of Eq. B gives

$$
\begin{aligned}
x(s) & =x_{0}+\left.x^{\prime}{ }_{0}\right|_{\frac{\lambda}{4}} ^{s}+\left.\left(\frac{\kappa z}{2}\right)\right|_{\frac{\lambda}{4}} ^{s}-\left.\frac{\kappa z}{2}[z \cos (k s)]\right|_{\frac{\lambda}{4}} ^{s}, \\
& =x_{0}+\left(x^{\prime}{ }_{0}+\frac{\kappa z}{2}\right)\left(s+\frac{\lambda}{4}\right)-\frac{\kappa z}{2}[z \cos (k s)] .
\end{aligned}
$$

Evaluating at $s=\frac{\lambda}{4}$, we then have

$$
x_{f}=x_{0}+\frac{\lambda}{2} x_{0}^{\prime}+\frac{\kappa \lambda}{4} z
$$

which becomes the second row of the transfer matrix. The third row of the $4 \mathrm{x} 4$ transfer matrix remains unchanged, $z_{f}=z_{0}$.

The final row of the transfer matrix is solved by substituting Eqs. and B.29 into Eq. , and evaluating at $s=\frac{\lambda}{4}$

$$
z_{f}=\kappa x_{0}+\frac{\lambda \kappa}{4} x_{0}^{\prime}+\frac{\kappa^{2} \lambda}{8} z
$$

Written as a transfer matrix for $x, x^{\prime}, z$, and $\delta$, and noting that the cavity length is half of a wavelength, $L=\frac{\lambda}{2}$, we then have the transfer matrix for a thick, one-cell cavity.

$$
M_{\text {thick-cell }}=\left[\begin{array}{cccc}
1 & L & \kappa \frac{L}{2} & 0 \\
0 & 1 & \kappa & 0 \\
0 & 0 & 1 & 0 \\
\kappa & \kappa \frac{L}{2} & \kappa^{2} \frac{L}{4} & 1
\end{array}\right]
$$

The z-dependence in the final momentum, which we refer to as the $R_{65}$ term of the full 6x6 matrix, is detrimental to several possible uses for a TDC, including 
LPS measurement and transverse-to-longitudinal emittance exchange. In a thin-cell approximation of $L=0$,

$$
M_{\text {thin-cell }}=\left[\begin{array}{cccc}
1 & 0 & 0 & 0 \\
0 & 1 & \kappa & 0 \\
0 & 0 & 1 & 0 \\
\kappa & 0 & 0 & 1
\end{array}\right]
$$

this thick-cavity term is zero. However, the length is non-negligible in an actual experiment, so we are interested in minimizing this effect. One solution is to use a multi-cell cavity. The total transfer matrix of several cells, each with a strength $\frac{\kappa}{N}$ and length $\frac{L}{N}$, is then $M_{\text {thick-cell }}^{N}$, and the full transfer matrix is

$$
M_{5-\text { cells }}=M_{\text {thick-cell }}^{5}=\left[\begin{array}{cccc}
1 & L & \kappa \frac{L}{2} & 0 \\
0 & 1 & \kappa & 0 \\
0 & 0 & 1 & 0 \\
\kappa & \kappa \frac{L}{2} & \frac{17}{100} \kappa^{2} L & 1
\end{array}\right] .
$$

which is identical to the single-cell expression aside from $a \sim \frac{1}{3}$ reduction in the thick-cell $R_{65}$ term. 
APPENDIX C

\section{SIMULATION CODE OVERVIEW}


A significant portion of the work performed for this dissertation was completed using a variety of well-and-lesser-known Accelerator Physics simulation codes. Due to the significant energy range present in the experiment (creation at the photocathode to $1 \mathrm{GeV}$ ), I had to stitch together the results of numerous simulations into one longer "start-to-end" simulation, which was frequently completed with the aid of GlueTrack. GlueTrack is the subject of Appendix D. In this appendix, I

present detailed overviews of many of the simulation codes used to perform the simulations of this dissertation, as well as more limited background information regarding related codes that were not extensively used, but are referenced briefly throughout the dissertation.

\section{C.1 Elegant}

ELEGANT [35] is the core of our simulation work. At one point its name was an acronym for "ELEctron Generation ANd Tracking", but after many updates its capabilities extend far past its original design [80], including particle types other than electrons, and the acronym has mostly been dropped. It is a fully $6 \mathrm{D}$ tracking code that has functionality for both transfer matrices or, when field profiles are known, tracking based on the actual EM fields. Most built-in elements have second order models included, which can be turned on individually or across the entire simulation.

A key feature of the code is that strengths are geometric rather than field strengths. For example, the bend angle is specified for a dipole, and particles of the central momentum $\left(p_{0}\right)$ are tracked at that angle, while other particles are 
tracked at the correct angle corresponding to their energy deviation from the reference momentum.

Here is a list of some of the ELEGANT elements used in our simulations. Elements neglect collective effects unless otherwise noted. The physics models that underlie these elements are described in Chapter 2, and their transfer matrices are discussed in Appendix A.

1. DRIFT: A simple drift over a specified length.

2. QUAD: A quadrupole magnet. The $\mathrm{K} 1$ value is the geometric bend for the $p_{0}$ parameter.

3. SBEND: A dipole bend. The bend angle and path length are specified, which is not standard across the codes discussed in this dissertation.

4. FITPT: A target for which optimization may be performed.

5. WATCH: A location at which to dump the simulation particle distribution as an SDDS file.

6. RFCA: An accelerating mode cavity that is modeled as a series of kicks.

7. RFDF: A deflecting mode cavity, as is used for the LPS diagnostics and the emtitance exchanger designs.

8. MATRIX: An arbitrary transfer matrix with user-defined terms. This was used as a variation of the accelerating mode cavities.

As far as our simulations are concerned, the most important capability of ELEGANT is its ability to optimize quadrupole parameters. This allows for the selection 
of optimal properties and locations of beamline elements or beam properties. ELEGANT's optimization algorithms are relatively simple, which is advantageous in determining an appropriate method, but can be finicky in terms of watching to make sure it can find a good solution rather than getting stuck in a "local best solution", i.e. a local minima of the optimizing function.

The optimization method has two relevant parts. First, an equation must be developed that is the figure of merit to be optimized for. Then, it will tweak the OPTIMIZATION_VARIABLE s simulation settings, and then uses a variable step-size to tweak select simulation settings to see what minimizes the target equation. For example, say we are trying to focus at a cross in the beamline, X114. We can try to focus to specific values of $\beta$ while modifying the strengths of quadrupole magnets Q106,Q107, and Q110, as an example of fitting the transverse dynamics into the low-energy bunch compressor.

$$
\begin{array}{r}
\text { optimization_term }=\left|\mathrm{X} 14 . \beta_{x}-20\right|+\left|\mathrm{X} 114 . \beta_{y}-30\right| \\
\text { optimization_variable }=\mathrm{Q} 106 . \mathrm{K} 1 \\
\\
\text { optimization_variable }=\mathrm{Q} 107 . \mathrm{K} 1 \\
\\
\text { optimization_variable }=\mathrm{Q} 110 . \mathrm{K} 1
\end{array}
$$

In this example, ELEGANT will calculate the optimization equation based on the default values of Q106,Q107, and Q110, and then test the value of the optimizing equation. It will then test nearby values of K1 for Q106,Q107, and Q110, and compare the result of the optimizing equation to determine the slope, to give an idea of what parameters it should try next. It will then tweak one or several of the $\mathrm{K} 1$ values based on specified min/max step sizes and recalculate the optimization 
equation, and make further tweaks based on its comparison to previous results. This procedure will be repeated until the optimization equation is smaller than a user-specified target value, or until it reaches a maximum number of iterations.

To avoid getting stuck in a local minima, ELEGANT can be commanded to try random restart positions, to see if it will converge to a better solution. However, this method still has its own shortcomings, and drastically different solutions can still be obtained by merely switching the initial values for the quadrupoles. For situations where convergence to the target value of the optimization equation is taking an exceedingly long time, it is useful to just switch the signs of the initial K1 values of the quadrupoles. This may have the effect of drastically shortening the time to reach better optimization values.

There are two main component files to Elegant simulations. First is the .LTE Lattice file that describes every single beamline element, including drifts, and organizes them into sequential LINES that describe sequential collections of the various elements. This structural organization will is relevant when we discuss various results, and how they were obtained.

The major downside of ELEGANT is that it does not handle collective effects adequately. It is often necessary to use a more appropriate simulation code that can handle the effects appropriately. For us, that code turned out to be IMPACT-Z.

\section{C.2 Impact-Z}

IMPACT-Z [75] is a z-domain fully 3-D tracking code that uses particle-in-cell and step-kick algorithms for modeling space charge effects. It allows for a variety of 
different beamline elements, including drifts, quadrupoles, dipoles, and field-mapbased RF cavities which were generated in HFSS.

In IMPACT-Z the space-charge (SC) interaction is modeled using a mean-field quasi-static particle-in-cell (PIC) algorithm and the (point-like) macroparticles are advanced through the beamline using high-order transfer maps. Each beamline element is segmented into axial slices modeled by transfer maps. Between each transfer-map segment, IMPACT-Z applies a space-charge "kick" evaluated from the mean-field PIC SC algorithm [75]. CSR effects are included in IMPACT-Z using the one-dimensional formulation described in Reference [17]. The one-dimensional model is valid provided [81]

$$
\mathcal{D}(s) \ll 1 \text {, with } \mathcal{D}(s) \equiv \frac{\sigma_{x}(s)}{\sigma_{z}(s)} \sqrt{\frac{\sigma_{x}(s)}{R(s)}} \text {, }
$$

where $R(s)$ is the trajectory's radius of curvature and $\sigma_{x}(s)$ and $\sigma_{z}(s)$ are respectively the transverse and longitudinal root-mean-square (RMS) sizes at the curvilinear beamline position $s$.

In IMPACT-Z the longitudinal charge distribution needed for the 1D CSR model is obtained from a longitudinal binning of the macroparticle ensemble. Convergence studies were carried out in order to determine the optimal number of longitudinal bins, $N_{z}$, to be used for both the SC and CSR calculations. Low values of $N_{z}$ typically underestimated the peak-current and therefore the collective effects, while large values of $N_{z}$ introduce numerical noise that can lead to artifacts . The convergence study [42] presented later in this Appendix revealed an appropriate value of $N_{z}=256$ for a bunch represented by $N=2 \times 10^{5}$ macroparticles. The number of bins in the transverse dimensions was set to $N_{x}=N_{y}=16$. The IMPACT-Z simulations presented in the rest of this paper use this set of parameters. 
IMPACT-Z has several simulation parameters that influence the computational intensity and physical accuracy of the simulations, including grid size for the SC and CSR calculations and the number of macroparticles used in each simulation. We needed to determine what parameters were sufficient to model the bunch compressor accurately without needlessly increasing the computational demand and time or suppressing small-scale features of the bunch. IMPACT-Z contains several dipole models, one that tracks only with space charge, and another that include an implementation of the 1-D model of steady-state CSR discussed above.

The first of the simulation parameters that we will discuss here is the number of bins to be used in the calculation of space charge effects, using a PIC algorithm. Having too few longitudinal bins, $N_{z}$, will smooth out the current distribution, reducing the effective peak current that drives emittance and energy spread growth, while having too many bins can introduce numerical noise that may lead to spurious effects. In addition, a large number of bins increases the computational time of the simulations.

For these tests, we adjusted the $N_{z}$ by powers of 2 , as required by IMPACT-Z which uses a FFT algorithm to convolve the charge distribution with the free-space Green's function. The bunch used for the testing is a generated three-dimensional Gaussian distribution with RMS parameters matched to the parameter computed for the "realistic" distribution generated by ASTRA [82]. The number of particles in each of the simulations was increased proportional to the number of bins such that there were, on average, 5 macroparticles per three-dimensional cell. With 16 cells in $x, 16$ cells in $y$, and 5 particles per cell, the total number of macroparticles $N$ for each of the simulations is shown in Table C.1. The value of $N_{z}$ was varied from 16 to 2048 and the corresponding simulations of BC1 included both the SC and CSR effects. 
Table C.1: Number of macroparticles $N$ used as a function of the number of longitudinal bins $N_{z}$.

\begin{tabular}{cc}
\hline$N_{z}$ & $N$ \\
\hline \hline 64 & 81920 \\
128 & 163840 \\
256 & 327680 \\
512 & 655360 \\
1024 & 1310720 \\
2048 & 2621440 \\
\hline
\end{tabular}

The number of particles to have an average of 5 particles per bin is the same order of magnitude as the number of particles that we have typically used in our simulations using realistic bunches (200k macroparticles). Given the lack of convergence in the emittance, there appears to be little benefit to increasing the $N_{z}$ above 256 bins. The bunch used for these tests is a generated Gaussian that has been matched to specified Courant-Snyder parameters and emittances, as the 200kmacroparticle distributions we export from ASTRA are insufficient for the type of parameter-optimizing performed here. The final LPS and current profiles are shown in Figures C.1 and C.2, respectively, and the variance of the current profiles for various $N_{z}$ compared to that of $N_{z}=64$ are shown in Figure fig:ImpactZcurrentVariance. The final horizontal normalized emittance $\varepsilon_{x}$ for these simulations are shown in Figure C.4.

The validity of these results is expected to depend on the precise distribution that is used. Because the beam is binned with spatial bins along the length of the bunch, distributions with long, sparsely populated tails may have different binnumber requirements to properly resolve their peak currents than more uniform and even distributions. 


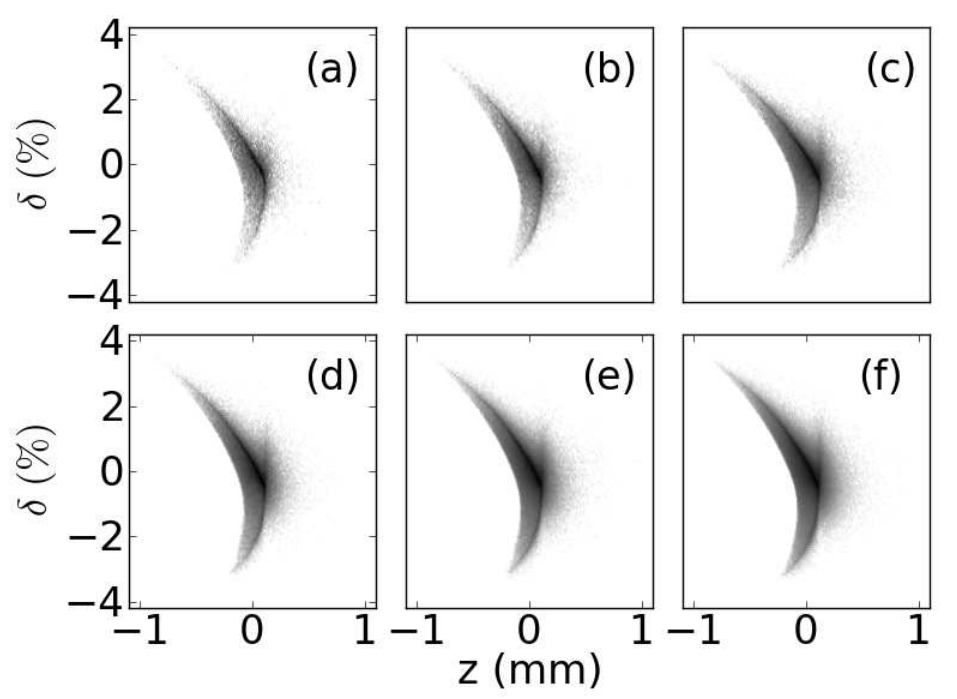

Figure C.1: LPS density plots at BC1 exit in IMPACT-Z varying the number of longitudinal space-charge bins $N_{z}$, while keeping the number of particles per bin constant to $N /\left(N_{x} N_{y} N z\right)=5$, with $N_{z}$ set to 64 (a), 128 (b), 256 (c), 512 (d), 1024 (e) and 2048 (f) bins. Adjusting the $N_{z}$ has little impact on the longitudinal dynamics for these simulations.

The other parameter that influences the simulations is the number of SC calculations done per beamline element. The sensitivity of the results on the number of SC kicks per element was found to be below $1 \%$ as long as we used four or more kicks per element. For this reason, our standard of four kicks per element is reasonable, and is used as the default value for the studies presented throughout this report.

\section{C.3 CSRtrack}

CSRTRACK is a code designed specifically to handle CSR effects as bunches pass through magnetic dipole fields. Due to its specialized and small-scale emphases (compared to the other tracking codes mentioned earlier), it uses a variety of different methods to track the particles. Presently, there are five different models 


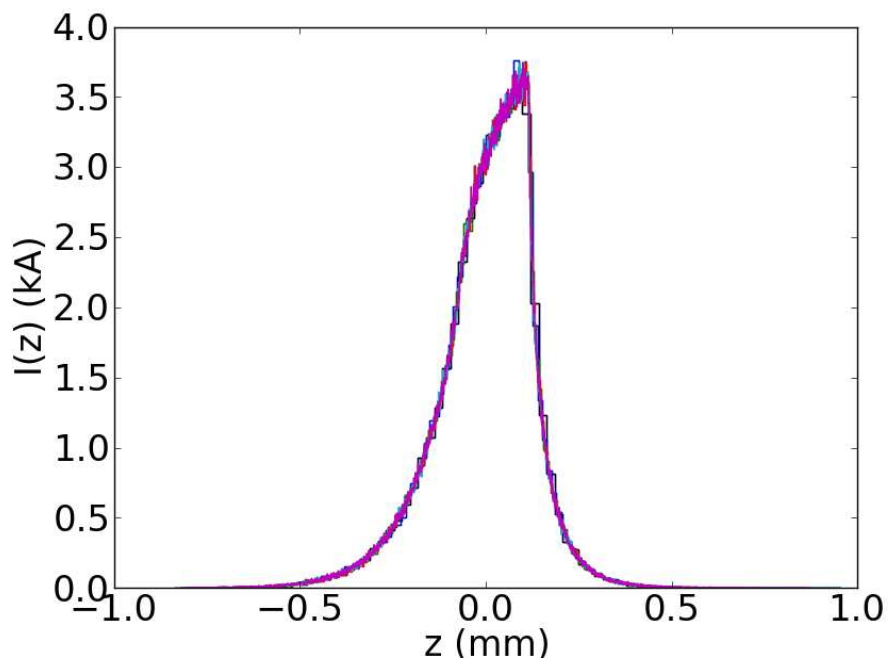

Figure C.2: Current profiles at $\mathrm{BC} 1$ exit in IMPACT- $\mathrm{Z}$ varying the number of longitudinal space-charge bins, while keeping the number of particles per bin constant by increasing the number of particles in the distribution, with $N_{z}$ set to 64 (red), 128 (blue), 256 (green), 512 (magenta), 1024 (yellow) and 2048 (black).

implemented, but we will only concern ourselves with three of them for now. All methods use an iterative tracking method with a variable time-step, that adjusts depending on what is determined necessary for the simulation. This can be overridden with either user-commands or by specifying an external list of time-step lengths, but we stuck with the built-in iterative method.

The most prominent model of CSRTRACK is the 2D particle-to-particle (P2P) model that directly computes the forces on macroparticles from the Liénard-Wiechart potentials evaluated at retarded times. These calculations are self-consistent and enable the computation of both the transverse and longitudinal force contributions from SC and CSR effects. The P2P model is computationally intensive, as it scales as $N^{2}$, where $N$ is the number of particles in the simulation. Due to the P2P model's computational intensity, CSRTRACK also includes an improved onedimensional model referred to as the 1D Projected (1DP) model. The 1DP model 


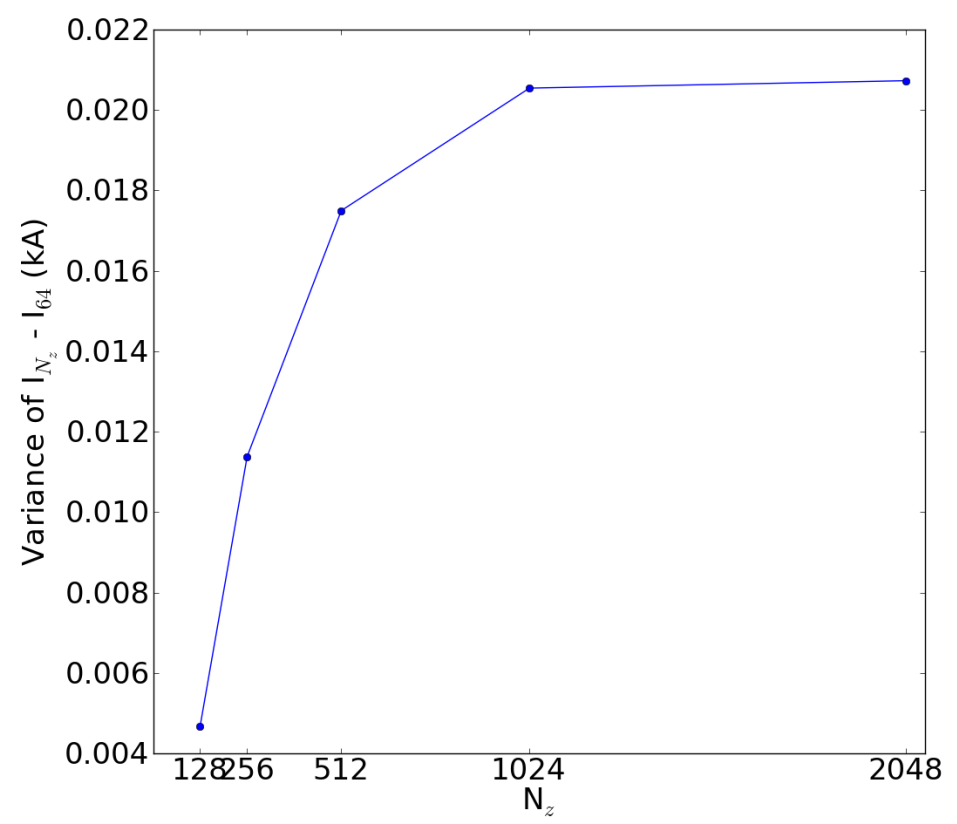

Figure C.3: Variance of $\mathrm{I}_{N_{z}}-\mathrm{I}_{64}$ for the data shown in FigureC.2, where $\mathrm{N}_{z}$ is the number of longitudinal bins. Compared to the scale of the peak current, this variance is well under $1 \%$.

uses the 1D projection of the smoothed charge distribution convoluted with a kernel function [83]. Compared to the model of Reference [17], the 1DP model is not limited to the ultra-relativistic regime.

CsRTRACK's P2P model treats each macroparticle as a 3D Gaussian charge distribution (referred to as "sub-bunch") in the $(x, y, z)$ space with distribution

$$
g(x, y, z)=\frac{1}{(2 \pi)^{3 / 2} \sigma_{h} \sigma_{v} \sigma_{\|}} e^{-\frac{x^{2}}{2 \sigma_{h}^{2}}-\frac{y^{2}}{2 \sigma_{v}^{2}}-\frac{z^{2}}{2 \sigma_{\|}^{2}}}
$$

where $\sigma_{h}, \sigma_{v}$ and $\sigma_{\|}$are respectively the horizontal, vertical and longitudinal RMS sizes of the sub-bunches. The resulting beam's spatial charge distribution is $\Phi(x, y, z)=$ $\sum_{j=1}^{N} Q_{j} g\left(x-x_{j}, y-y_{j}, z-z_{j}\right)$ where $Q_{j}$ and $\left(x_{j}, y_{j}, z_{j}\right)$ are respectively the $i$-th sub-bunch charge and LPS coordinates. In CSRTRACK, $\sigma_{v}$ and $\sigma_{\|}$may be defined 


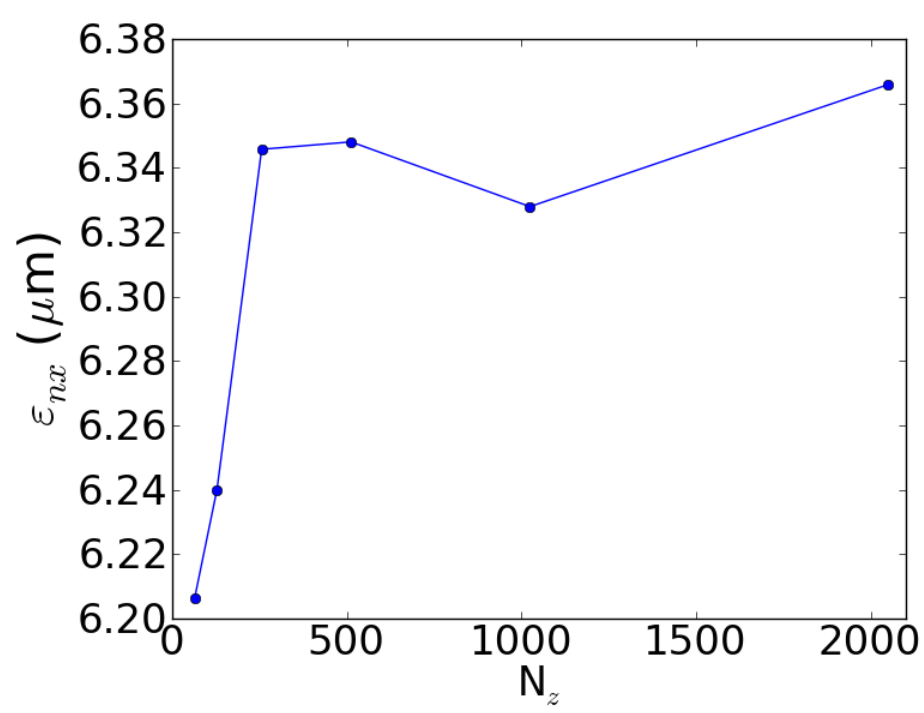

Figure C.4: $\varepsilon_{x}$ BC1 exit in ImPACT-Z varying the number of longitudinal spacecharge bins, $N_{z}$, while keeping the number of particles per bin constant, with $N_{z}$ set to $64,128,256,512,1024$ and 2048 number of bins.

relative to the vertical and longitudinal RMS bunch sizes, $\sigma_{y}$ and $\sigma_{z}$, respectively, and are adjusted along the bunch compressor as the dimensions of the bunch change. The "Vertical" dimension ( $z$ in CSRTRACK and $y$ in our standard notation) does not receive any force from collective effects in these models.

CSRTRACK includes a model known as "Particle-to-Grid" that is partially analogous to the "Particle-in-Cell method" used by IMPACT-Z, with the field calculated for each spatial cell in a gridded-space, and the specific value of the fields for each particle interpolated from there. We did not use this method for our simulations.

While tracking 200,000 macroparticles in ELEGANT and IMPACT-Z is entirely feasible and achievable within reasonably time constraints, a full particle-to-particles CSRTRACK simulation for either of the bunch compressors would take prohibitively long. This resulted in simulations with particles on the order of $1 \times 10^{5}$, around an order of magnitude smaller than the other simulation codes despite taking many 
times longer to complete. A study to choose the parameters is presented in a later section of this Appendix.

\section{C.4 CSRtrack Parametric Studies}

Using either CSRTRACK's P2P solver or its 1-D Projected model, the relevant simulation parameters (beyond basic descriptions of the dipoles and layout of the chicane) are the number and the size of the Gaussian sub-bunches, $\sigma_{B}$, used to represent the three-dimensional distribution. Due to the drastic increase in run time when using the P2P model, e.g, compared to IMPACT-Z, the studies presented here needed to be significantly more focused in scope. The sub-bunch size determines the susceptibility to numerical noise. Going for this goal of distribution-smoothing likely dampens out any microbunching instabilities that could manifest in more complete simulations. However, accurately resolving microbunching instabilities would be far more time and computationally intensive than those used for the studies presented in this thesis.

We used Fourier analysis as quick estimates of what Gaussian sizes were appropriate. This entailed summing every Gaussian sub-bunch over each projected axis to recreate the effective, smoothed distribution, using a method similar to that performed internally by CSRTRACK. We then performed Fourier analysis to inspect for high-frequency noise, which would manifest itself in simulations as spurious forces. In cases where $\sigma_{B}$ is much smaller than the average inter-particle spacing, the Fourier transformation would reveal noise. At the other extreme, where $\sigma_{B}$ is on the order of the bunch length $\sigma_{z}$, the effective length of the bunch is increased, which erroneously reduces space-charge and CSR effects. This led to the general procedure of trying 
to determine the shortest possible Gaussian that does not suffer from spurious noise in the Fourier analysis.

We were also concerned that the focusing and compression would change the requirement as the bunch passes through the compressor. Fortunately, CSRTRACK allows for setting the Gaussian size as a variable of the bunch size at each time step. This is only applicable to the longitudinal and vertical dimension, and not the dimension of the bend. For the horizontal dimension, we chose it based on the final horizontal RMS size, as both CSR and SC effects are greater when the bunch lengths are shorter. The number of sub-bunches was then chosen to insure the transverse beam density does not display significant numerical noise.

With CSRTRACK's 1-D Projected model, we used 200k particles, so the required Gaussian size needed to eliminate spurious noise was much smaller than the 3D simulations, which used $5 \mathrm{k}$ to $30 \mathrm{k}$ particles. In Table C.2, we present the final emittances and energy spreads for simulations using 200k particles, and variable Gaussian sub-bunches as some fraction of the total RMS length.

A Gaussian sub-bunch length of approximately $10 \%$ of the total RMS bunch size in $z$ and $y$ and $0.01 \mathrm{~mm}$ in $x$ was found to be appropriate for $10 \mathrm{k}$ particles. This then raises the question of what is appropriate when increasing and/or decreasing the size based on the number of particles used.

When the sub-bunch size is around $0.1 \%$ of the RMS length or less, CSRTRACK's calculation fails to converge and the forces are not solved correctly, leading to erratic and/or outright broken behavior in which the CSR effects are not calculated correctly. This presents itself in outgoing emittances that are nearly what one would achieve with a single-particle (no CSR or LSC) simulation, as shown in Figure C.5. This corresponds to when the Gaussian size is comparable to or smaller than the average inter-particle spacing, or 
Table C.2: Scan of Variable Size

\begin{tabular}{lccc}
\hline \% RMS length & $\varepsilon_{x}$ & $\delta$ & $I_{\text {peak }}$ \\
\hline \hline $100 \%$ & 22.5 & 1.49 & 6.81 \\
$50 \%$ & 36.8 & 1.55 & 6.95 \\
$30 \%$ & 44.6 & 1.67 & 7.85 \\
$20 \%$ & 50.7 & 1.78 & 8.20 \\
$15 \%$ & 53.3 & 1.81 & 8.4 \\
\hline $10 \%$ & 54.9 & 1.83 & 8.04 \\
$5 \%$ & 54.5 & 1.87 & 8.78 \\
$1 \%$ & 55.3 & 1.87 & 7.73 \\
$0.5 \%$ & 55.4 & 1.87 & 7.11 \\
\hline
\end{tabular}

$$
\langle r\rangle \approx 1 / n^{1 / 3}
$$

where $n \equiv N /\left(\sigma_{x} \sigma_{y} \sigma_{z}\right)$ is the macroparticle density. For a 3.2-nC bunch with the expected range of betatron functions and emittances, this is on the order of 0.1 mm. When $\sigma_{B}$ is on the order of $\sigma_{z}$, the effective bunch length is increased and the emittance growth due to CSR is reduced. Values for $\sigma_{B}$ in the range of around $1 \%-10 \%$ of the RMS bunch length suffer from neither issue, and thus $5 \%$ of $\sigma_{z}$ is chosen to be the baseline for our 1DP model simulations.

For 3D simulations, such a comprehensive scan was too demanding, so we performed scattered simulations, with several different numbers and sizes for the Gaussian sub-bunches. Table 4.3 shows the results for the simulation models considered in this report. The settings and parameters for a 3.2-nC bunch with linearized LPS are shown. 


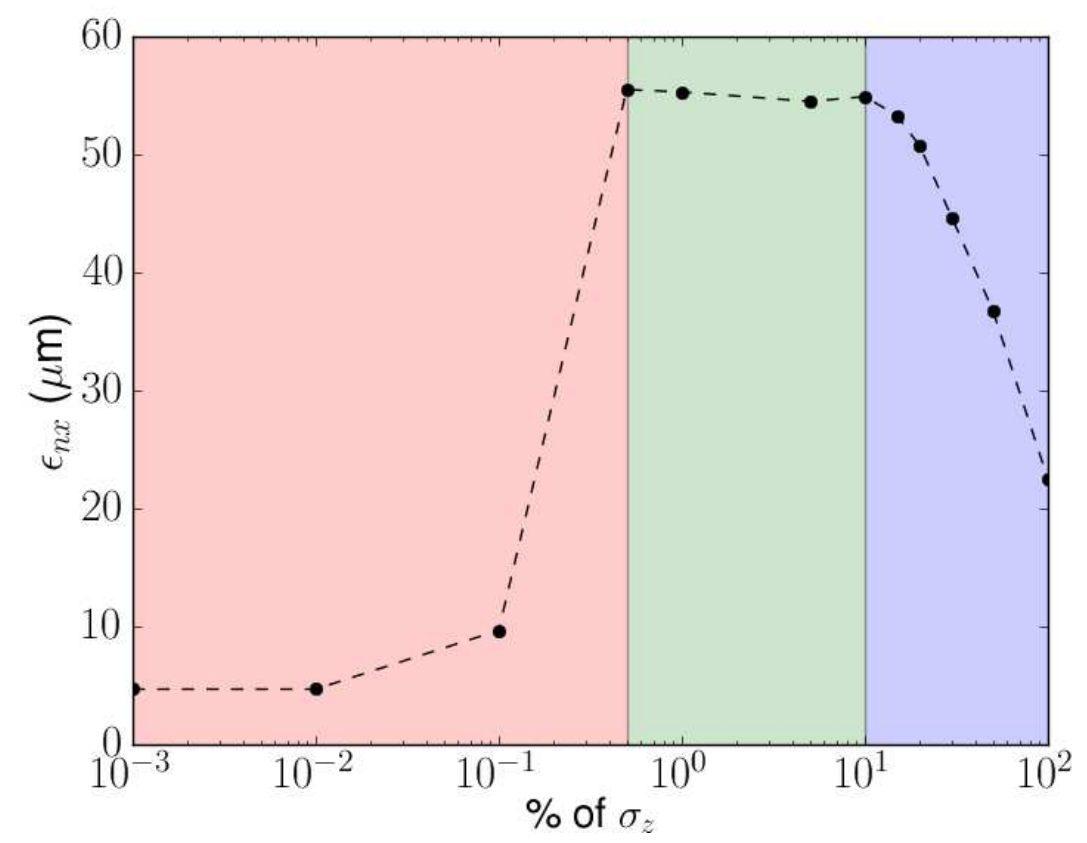

Figure C.5: $\varepsilon_{x}$ BC1 exit in CSRTRACK with the 1DP model versus the size of the Gaussian sub-bunches as a fraction of the RMS length, $\sigma_{z}$. The blue-shaded region corresponds to where $\sigma_{B} \sim \sigma_{z}$, which increases the effective bunch length and decreases the effects of the CSR. The green-shaded region corresponds to where $\sigma_{B}$ is sufficiently small to not increase the effective bunch length, which gives consistent results. The red-shaded region corresponds to where $\sigma_{B}<<\sigma_{z}$ and on the order of the average inter-particle separation, which causes CSRTRACK's solver to fail to converge resulting in an underestimation of the impact of CSR.

\section{C.5 Impact-T}

IMPACT-T is the time-domain alternative (and in fact, original basis) for IMPACTZ. The time-domain space-charge method is more accurate for low-energy particles, SO IMPACT-T is a viable alternative for simulating the creation of the beam. More importantly, it doesn't have the shortcomings with regards to asymmetric beams that ASTRA suffers from, which makes it ideal for the generation of flat beams. Unlike IMPACT-Z, it allows for magnetic fields from various sources to overlap each 
other, which is relevant to the generation of flat beams. There is little benefit to using IMPACT-T after the beam has been accelerated to $50 \mathrm{MeV}$ for a $3.2 \mathrm{nC}$ beam, for which IMPACT-Z is a faster substitute.

\section{C.6 Comparison of Code Capabilities}

In Table C.3, I present parameters for the various simulation modes, and what effects they account to for our simulations. There are additional modes that were not used for this dissertation, due to their limitations. The CSR and SC forces are subdivided into transverse and longitudinal, with $\mathrm{T}$ and L, respectively.

Table C.3: Initial transverse emittances for the charges used.

\begin{tabular}{ccccccc}
\hline Code & Model & Abbr. & $C S R_{L}$ & $C S R_{T}$ & $S C_{L}$ & $S C_{T}$ \\
\hline \hline ELEGANT & Tracking & Ele & $\times$ & $\times$ & $\times$ & $\times$ \\
IMPACT-Z & SC & SC & $\times$ & $\times$ & $\circ$ & $\circ$ \\
IMPACT-Z & SC+CSR & SC+CSR & $\circ$ & $\times$ & $\circ$ & $\circ$ \\
CSRTRACK & 1D-Proj. & 1DP & $\circ$ & $\times$ & $\times$ & $\times$ \\
CSRTRACK & Part.-to-Part. & P2P & $\times$ & $\times$ & $\circ$ & $\circ$ \\
\hline
\end{tabular}

\section{C.7 Comparison of Particle Formats}

Due to the complex combination of different simulations and formats that must be utilized to perform S2E simulations, keeping track of the different particle formats is a necessity. In Table C.4, I present each of the particle formats that is used for the simulations presented in this dissertation or are compatible with GLUETRACK 
(see Appendix D for more details). The table includes code, format, and a brief description.

Table C.4: Descriptions of particle formats for inter-code compatibility

\begin{tabular}{ccc}
\hline Code & Format & Description \\
\hline \hline Elegant & SDDS & Self-described Data Structure \\
Impact-Z & -2 & Custom dump that is in the same units as the input \\
CSRtrack & FMT1 (no ref.) & Description of bunch without reference particle \\
CSRtrack & FMT1 (ref.) & Description of bunch with reference particle \\
GlueTrack & xxs & Internal GlueTrack format, dimension and slopes \\
GlueTrack & xpx & Internal GlueTrack format, dimension and momenta \\
\hline
\end{tabular}

These simulation codes and particle formats are referenced widely throughout this paper.

\section{C.8 Simulation Outline}

Due to the varying effects that must be accounted for in full start-to-end simulations along with the inability of any single code to model the full beamline, we link together several different simulations to design and model the full particle accelerator. A sample diagram of this flow is shown in Figure C.6

More details on GLUETRACK and S2E simulations are presented in Appendix D. 


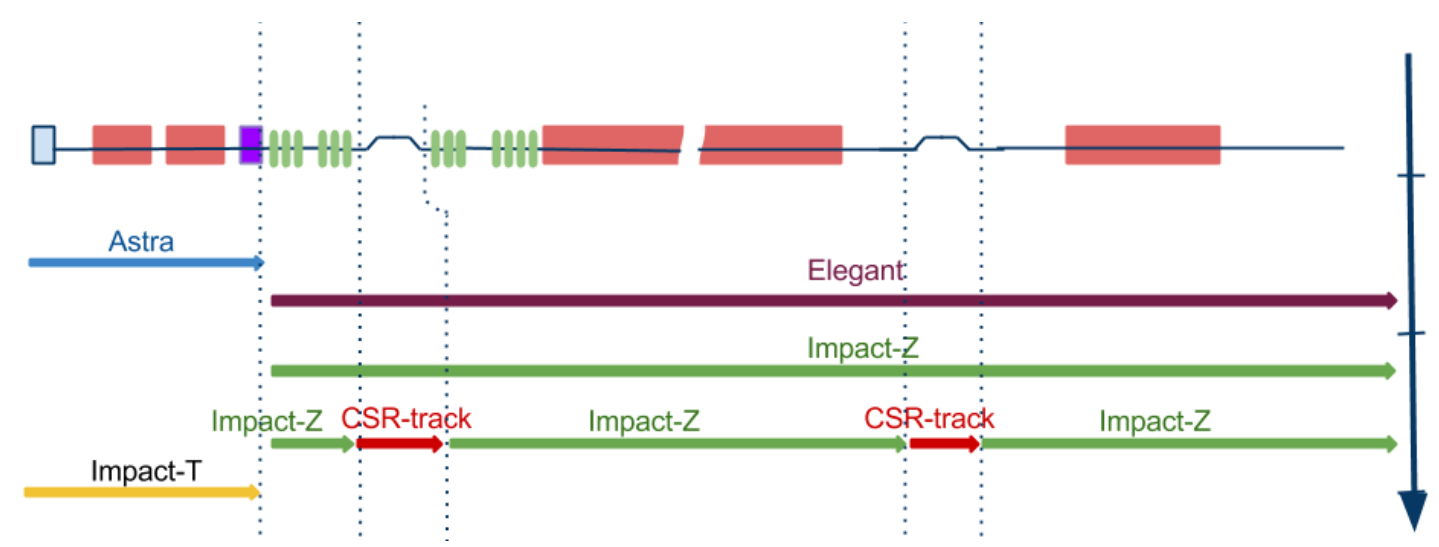

Figure C.6: Schematic of ASTA denoting which simulation codes are used for different sections. 


\section{APPENDIX D}

\section{GLUETRACK: FRAMEWORK FOR MULTI-CODE SIMULATIONS}


Beam dynamics simulations of entire particle accelerators from start-to-end (S2E) usually require the use of multiple simulation programs. This is due to the range of energies and bunch dimensions that influence the validity of numerical models. For

example, transverse space charge (SC) forces, due to their $\frac{1}{\gamma^{2}}$ dependence, are vastly more important at low energies, such as before $\mathrm{CC} 1$ and $\mathrm{CC} 2$, that a more detailed and computationally intensive SC algorithm is required, such as that in ASTRA or IMPACT-T. However, these codes are slower, and are not feasible for modeling a full $100 \mathrm{~m}$ simulation.

Originally obtained from Igor Zogorodnov from DESY in 2006 [84], GLuETRACK is a set of Python scripts designed to manage the interaction and analysis of multi-code simulations, and includes many manipulation methods to act directly on bunches. Due to the extensive code development I performed over the years of my Ph.D. research, and this code's eventual use for ASTA as a user's facility, this chapter is its own section/appendix, wherein I detail the overall flow of a GLUETRACK S2E simulation, and describe my additional functions.

\section{D.1 GlueTrack Flow Overview}

GlueTrack's workload is divided across four primary files.

1. The "main" file, which specifies the input deck that lists the commands, as well as working directories.

2. The input deck, which lists the commands to be acted on in GlueTrACK. A more detailed explanation of its structure follows later.

3. The S2E_TOOLS.PY, which parses the input deck. 
4. PARTICLE_TOOLS.PY, which defines functions to be called by S2E_TOOLS.

To date, the codes that we have implemented with use in GLUETRACK are:

\section{IMPACT-T}

\section{IMPACT-Z}

\section{Elegant}

\section{CSRTRACK}

More detailed descriptions of these codes can be found in Appendix C.

In addition to being designed to launch simulations, there are even more particle formats that GLUETRACK recognizes for reading and writing functions:

\section{ASTRA}

2. IMPACT-T

3. ImPACT-Z (35 input, -2 output).

4. ELEGANT's SDDS format.

5. CSRTRACK's FMT1, with or without reference particle data as the 2nd line.

6. $x x s$, an internal format for GlueTrack with $x, x^{\prime}, y, y^{\prime}, z, \delta$

7. $x p x$, an internal for GlueTrack, with $x, p_{x}, y, p_{y}, z, p_{z}$

A GlueTrack input command list is a hierarchical list of instructions for GlueTrack to perform, which is read by a built-in parser. These commands, in descending order, are 
1. SECTIOns, demarcated by \#00, \#01, \#02, etc., which divide the input file into distinct sets of commands.

2. ACTIONs, either PROCESSOR, ANALYZE, or the name of a code, which tells GLUETRACK what sets of tasks are valid.

3. TASKs, which are individual specific processes the user wants GlueTrack to perform.

4. PROPERTIES, which are further instructions and values for GlueTrack to use, such as executable directories, file paths, simulation input files, and transformation parameters.

As an example, a simple input file (corresponding to simulations presented in Chapter 4, is listed here as an example.

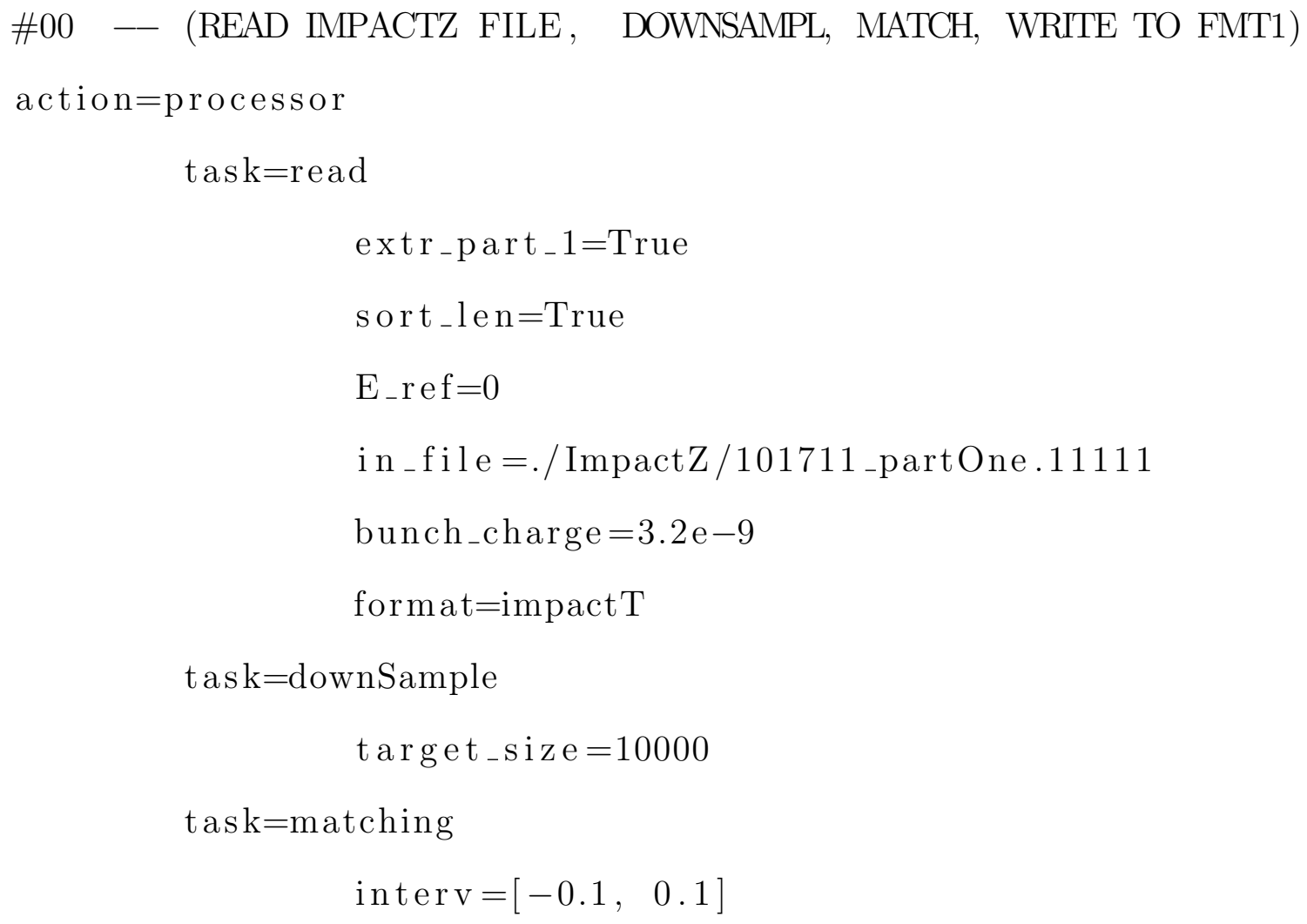




$$
\begin{aligned}
& \text { xpar }=\left[\begin{array}{lll}
3.0, & 8.0, & 0.00
\end{array}\right] \\
& \text { ypar }=\left[\begin{array}{lll}
0.0, & 0.0, & 0.0
\end{array}\right]
\end{aligned}
$$

action $=$ processor

$$
\begin{aligned}
& \text { task=write } \\
& \qquad \begin{array}{l}
\mathrm{z} 0=0.0 \\
\text { out_file }=. / \text { CSRtrack } / \text { in } / \mathrm{xUBC} 1 . \mathrm{fmt} 1 \\
\text { format }=\mathrm{fmt} 1 \mathrm{refPart}
\end{array}
\end{aligned}
$$

\#01 RUN CSRTRACK - - (LAUNCHES THE CSRTRACK SIMULATIONS) action $=$ CSRTrack

$$
\text { task=run }
$$

$$
\text { command }=. / \text { CsrTrack } 10
$$

work_dir=CSRtrack /

deck_in $=$ BC1_CSRtrack_1e4. in

deck_in $\mathrm{p}=\mathrm{BC} 1 \_$CSRtrack . in

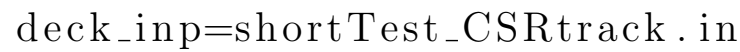

dist_in $p=$. / CSRtrack/in / xUBC1.fmt1

\#02 ANALYZE - - (TAKES THE CSRTRACK SIMULATION DATA AND ANALYZES IT FOF

action=analys is

$$
\text { task=read }
$$

extr_ $_{-}$part_ $1=$ True

sort_len=True

E_ref $=0$

in f file =./CSRtrack/out/end.fmt3

format $=\mathrm{fmt} 3$

action=analys is 


$$
\begin{aligned}
& \text { task=slice } \text { analysis_t }_{-} o_{-} \text {file } \\
& \text { M_av }=100000 \\
& \text { output_str }=\text {./ScanResults / scanTwissData_CSRon_3_8_1e4 } \\
& \text { interv }=[-0.003,0.003] \\
& \text { initial_alpha }=3 \\
& \text { initial_beta }=8 \\
& \text { task=m_combo_write } \\
& \text { savefile }=\text {./ScanResults / sliceData_CSRon_3_8_1e4 } \\
& \operatorname{sigmaI}=.1 \mathrm{e}-6\}
\end{aligned}
$$

This input file reads an IMPACT-T file, downsamples it to ten-thousands particles, matches the C-S parameters to specific values, then writes it to a fmt1 file for use in CSRTRACK. GlueTRACK then runs a specified CSRtrack lattice with a specified distribution, and lastly performs a slice analysis on CSRtrack's output file.

An S2E simulation may be far more complicated, involving one CODE action and one processor action of each distinct section.

\section{D.2 Modifications}

While GlueTrack contains dozens of tasks and functions, there are many that I specifically developed to help in the S2E simulations for ASTA. These functions include:

1. ELEGANT, Basic compatibility

2. SDDSREAD, CUSTOMsdds format for ELEGANT reading functions.

3. CSRTRACK, for running CSRtrack 
4. ImpACT-Z, for running impact-Z

5. IMPACT-Z read/write functions for IMPACT-Z

6. MASKING_STATEMENT_XXS, which allows arbitrary collimation based on the phase space parameters.

7. LPSPlot, PlotShow, etc. for quick diagnostics.

\section{D.3 Start-to-End Simulations}

One of the major appeals of using GLUETRACK is its simplification of start-toend simulations. As shown in Figure D.1, we use different codes for different parts of the beamline. The gun is modeled in ASTRA for its detailed SC calculations. The full beamline and its quadrupole magnet strengths are modeled and optimized in ELEGANT. The other codes are used to model the full beamline with different models of SC and CSR. Interstitial processing and beam manipulations are implemented using GlueTrack. These include collimations, down sampling the number of particles, linear and non-linear transformations of the beam phase space, and more. 


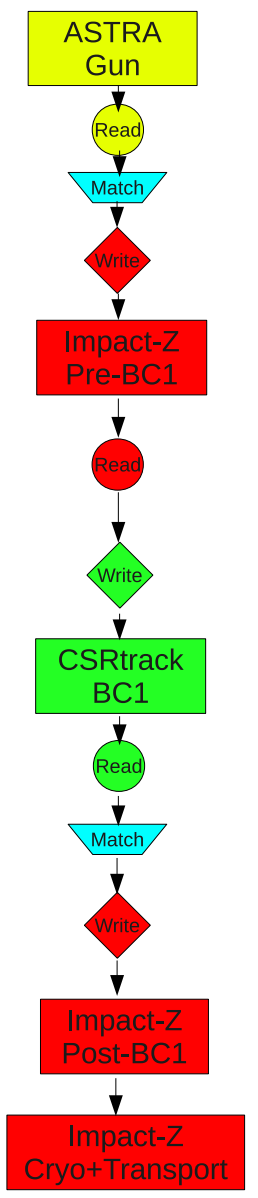

Figure D.1: Schematic of a start-to-end simulation using GlueTrack. This simulation uses five simulations, three read-write pairs, and two "matching" processes. 